Supporting Information for:

\title{
A Joint Experimental and Theoretical Study of the Palladium Catalyzed Electrophilic Allylation of Aldehydes
}

\author{
Olivier Piechaczyk, Thibault Cantat, Nicolas Mézailles, Pascal Le Floch* \\ Laboratoire « Hétéroéléments et Coordination », École Polytechnique, CNRS, 91128 Palaiseau, \\ France.E-mail : lefloch@poly.polytechnique.fr
}

Experimental Section 3

General remarks. 3

DFT Data 3

I. Non catalysed reaction 3

With formaldehyde

Optimized geometry, SCF energy, three lower frequencies, thermochemistry and PCM energy for formaldehyde. 3 Optimized geometry, SCF energy, three lower frequencies, thermochemistry and PCM energy for allyltrimethyltin

Optimized geometry, SCF energy, three lower frequencies, thermochemistry and PCM energy for $\mathbf{T S}_{\alpha} \ldots \ldots \ldots \ldots \ldots . .5$

Optimized geometry, SCF energy, three lower frequencies, thermochemistry and PCM energy for $\mathbf{T S}_{\gamma} \ldots \ldots \ldots \ldots . . .6$

Optimized geometry, SCF energy, three lower frequencies, thermochemistry and PCM energy for product...... 8



Optimized geometry, SCF energy, three lower frequencies, thermochemistry and PCM energy for bromobenzaldehyde

Optimized geometry, SCF energy, three lower frequencies, thermochemistry and PCM energy for $\mathbf{T S}_{\gamma} \ldots \ldots \ldots . . . .10$

Optimized geometry, SCF energy, three lower frequencies, thermochemistry and PCM energy for product..... 12

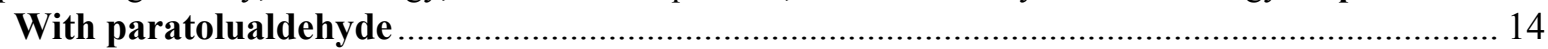

Optimized geometry, SCF energy, three lower frequencies, thermochemistry and PCM energy for

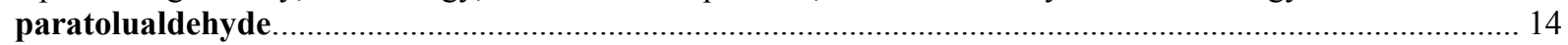

Optimized geometry, SCF energy, three lower frequencies, thermochemistry and PCM energy for $\mathbf{T S}_{\gamma} \ldots \ldots \ldots \ldots . .15$

Optimized geometry, SCF energy, three lower frequencies, thermochemistry and PCM energy for product..... 16

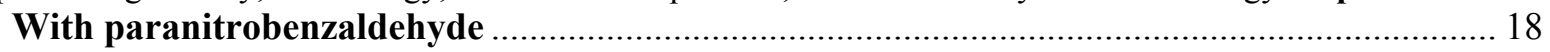

Optimized geometry, SCF energy, three lower frequencies, thermochemistry and PCM energy for

paranitrobenzaldehyde...

Optimized geometry, SCF energy, three lower frequencies, thermochemistry and PCM energy for $\mathbf{T S}_{\gamma} \ldots \ldots \ldots . . .19$

Optimized geometry, SCF energy, three lower frequencies, thermochemistry and PCM energy for product..... 21

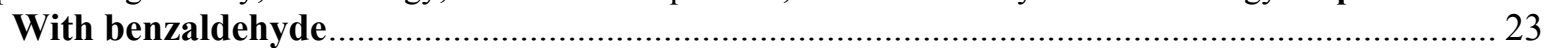

Optimized geometry, SCF energy, three lower frequencies, thermochemistry and PCM energy for

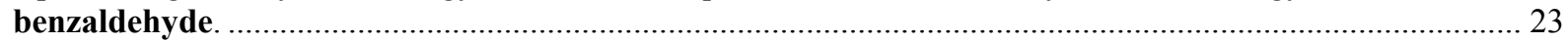

Optimized geometry, SCF energy, three lower frequencies, thermochemistry and PCM energy for $\mathbf{T S}_{\gamma} \ldots \ldots \ldots . . .24$

Optimized geometry, SCF energy, three lower frequencies, thermochemistry and PCM energy for product..... 25



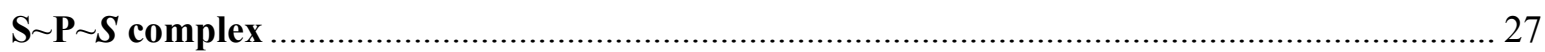

Optimized geometry, SCF energy, three lower frequencies, thermochemistry and PCM energy for A1 ............. 27

Optimized geometry, SCF energy, three lower frequencies, thermochemistry and PCM energy for $\mathbf{T S}_{\mathbf{A 1 A 2}} \ldots . . .29$

Optimized geometry, SCF energy, three lower frequencies, thermochemistry and PCM energy for A2 ............ 30 
Optimized geometry, SCF energy, three lower frequencies, thermochemistry and PCM energy for $\mathbf{T S}_{\mathbf{A 2 A 1}} \cdots \ldots . . .32$

Optimized geometry, SCF energy, three lower frequencies, thermochemistry and PCM energy for A3. .......... 34

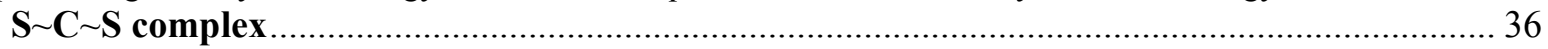

Optimized geometry, SCF energy, three lower frequencies, thermochemistry and PCM energy for A1. ............ 36

Optimized geometry, SCF energy, three lower frequencies, thermochemistry and PCM energy for $\mathbf{T S}_{\mathbf{A 1 A 2}} \ldots . . .38$

Optimized geometry, SCF energy, three lower frequencies, thermochemistry and PCM energy for A2 ........... 39

Optimized geometry, SCF energy, three lower frequencies, thermochemistry and PCM energy for $\mathbf{T S}_{\mathrm{A2A1}} \ldots \ldots . .41$

Optimized geometry, SCF energy, three lower frequencies, thermochemistry and PCM energy for A3...........43

Optimized geometry, SCF energy, three lower frequencies, thermochemistry and PCM energy for allylalcoolate.

III. Lewis acid mechanism

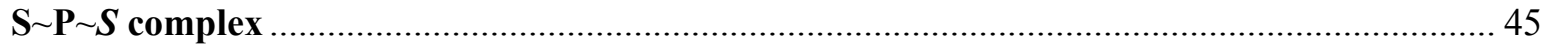

Optimized geometry, SCF energy, three lower frequencies, thermochemistry and PCM energy for B0.............46

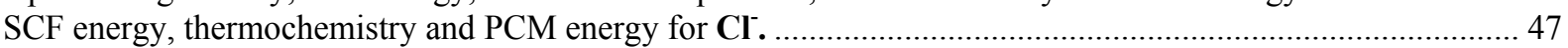

Optimized geometry, SCF energy, three lower frequencies, thermochemistry and PCM energy for B1 ............47

Optimized geometry, SCF energy, three lower frequencies, thermochemistry and PCM energy for B2............ 48

Optimized geometry, SCF energy, three lower frequencies, thermochemistry and PCM energy for $\mathbf{T S}_{\mathbf{B} 2 \mathbf{B} 3} \cdot \ldots . .50$

Optimized geometry, SCF energy, three lower frequencies, thermochemistry and PCM energy for B3............ 52



Optimized geometry, SCF energy, three lower frequencies, thermochemistry and PCM energy for B0............ 54

Optimized geometry, SCF energy, three lower frequencies, thermochemistry and PCM energy for B1............55

Optimized geometry, SCF energy, three lower frequencies, thermochemistry and PCM energy for B2 .............56

Optimized geometry, SCF energy, three lower frequencies, thermochemistry and PCM energy for $\mathbf{T S}_{\mathbf{B} 2 \mathbf{B} 3} \ldots \ldots .57$

Optimized geometry, SCF energy, three lower frequencies, thermochemistry and PCM energy for B3............ 59

Optimized geometry, SCF energy, three lower frequencies, thermochemistry and PCM energy for $\mathbf{T S}_{\mathbf{B} 3 \mathbf{B} 2} \cdot \ldots \ldots 61$

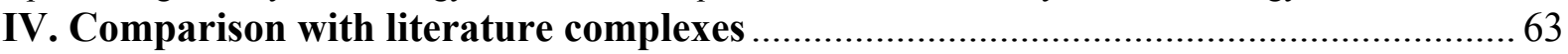

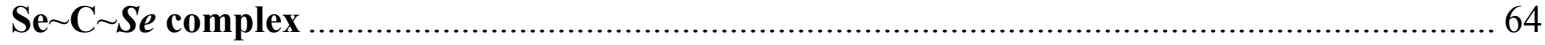

Optimized geometry, SCF energy, three lower frequencies, thermochemistry and PCM energy for B2 .............64

Optimized geometry, SCF energy, three lower frequencies, thermochemistry and PCM energy for $\mathbf{T S}_{\mathbf{B} 2 \mathbf{B} 3} . \ldots . .65$

Optimized geometry, SCF energy, three lower frequencies, thermochemistry and PCM energy for B3............6 67

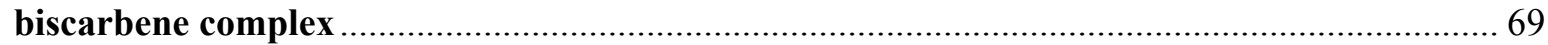

Optimized geometry, SCF energy, three lower frequencies, thermochemistry and PCM energy for B2 .............69

Optimized geometry, SCF energy, three lower frequencies, thermochemistry and PCM energy for $\mathbf{T S}_{\mathbf{B} 2 \mathbf{B} 3} \ldots \ldots .70$

Optimized geometry, SCF energy, three lower frequencies, thermochemistry and PCM energy for B3.............73






\section{Experimental Section}

\section{General remarks}

All reactions were performed under an inert atmosphere of argon or nitrogen by using Schlenk and glove-box techniques and dry deoxygenated solvents. Dry THF, ether and hexanes were obtained by distillation from $\mathrm{Na} /$ benzophenone. Dry dichloromethane was distilled on $\mathrm{P}_{2} \mathrm{O}_{5}$ and dry toluene on $\mathrm{Na}$. Nuclear magnetic resonance spectra were recorded on a spectrometer operating at $300.0 \mathrm{MHz}$ for ${ }^{1} \mathrm{H}$. Solvent peaks are used as internal reference relative to $\mathrm{Me}_{4} \mathrm{Si}$. Complexes $\mathbf{1}$ and $\mathbf{2}$ were prepared according to literature procedures (Doux, M.; Mezailles, N.; Melaimi, M.; Ricard, L.; Le Floch, P. Chem. Commun. 2002, 1566-1567, Chentit, M., PhD Thesis, Faculté des sciences de l'Université de Genève: Geneva, 1999).

\section{DFT Data}

\section{Non catalysed reaction}

\section{With formaldehyde}

Optimized geometry, SCF energy, three lower frequencies, thermochemistry and PCM energy for formaldehyde.

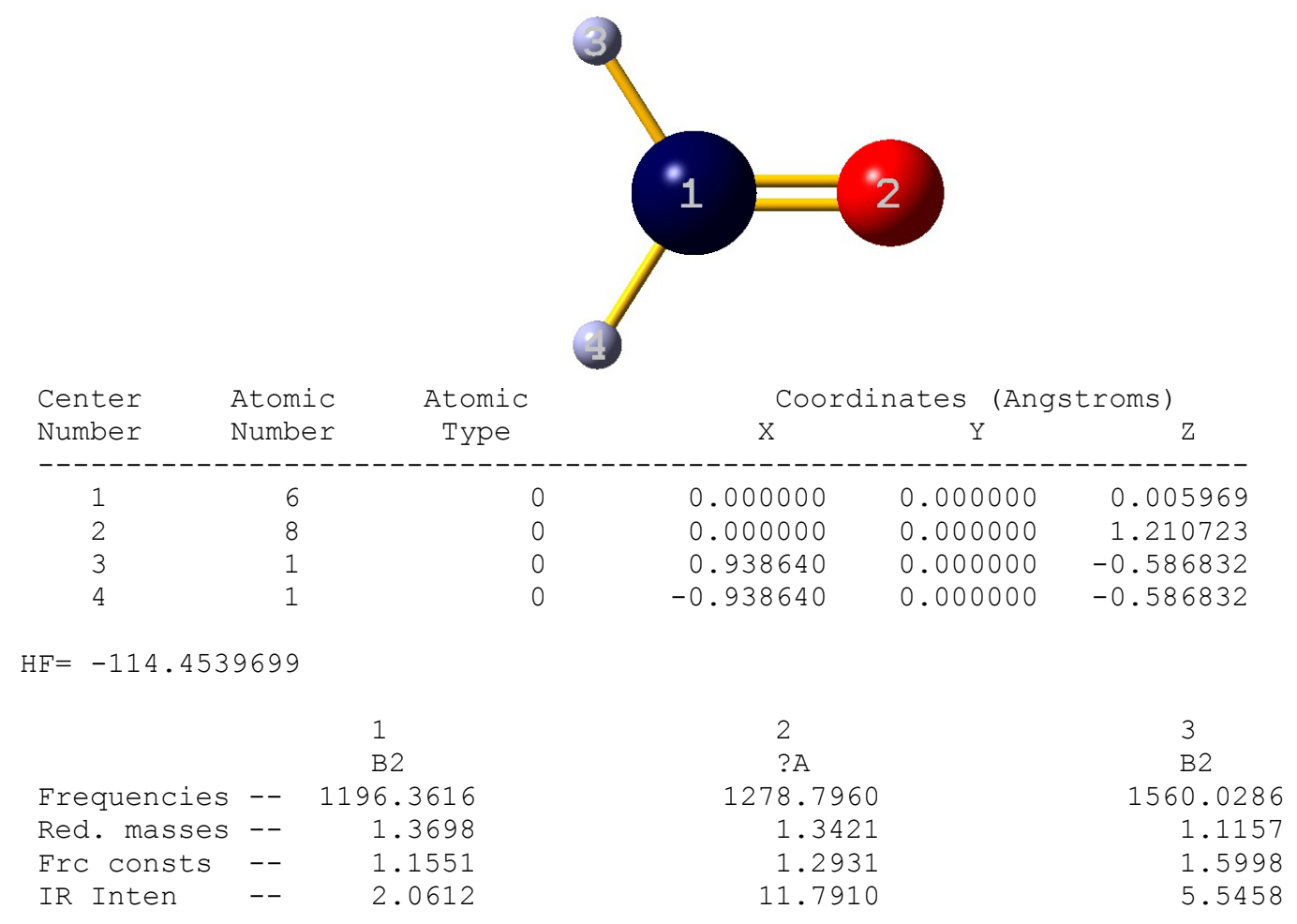




\begin{tabular}{crccccccrrr} 
Atom & AN & $\mathrm{X}$ & $\mathrm{Y}$ & $\mathrm{Z}$ & $\mathrm{X}$ & $\mathrm{Y}$ & $\mathrm{Z}$ & \multicolumn{1}{c}{$\mathrm{X}$} & \multicolumn{1}{c}{$\mathrm{Y}$} & $\mathrm{Z}$ \\
1 & 6 & 0.00 & 0.17 & 0.00 & 0.15 & 0.00 & 0.00 & 0.00 & 0.00 & -0.01 \\
2 & 8 & 0.00 & -0.04 & 0.00 & -0.08 & 0.00 & 0.00 & 0.00 & 0.00 & 0.08 \\
3 & 1 & 0.00 & -0.70 & 0.00 & -0.25 & 0.00 & -0.65 & -0.35 & 0.00 & -0.61 \\
4 & 1 & 0.00 & -0.70 & 0.00 & -0.25 & 0.00 & 0.65 & 0.35 & 0.00 & -0.61
\end{tabular}

thermodynamics:

Sum of electronic and zero-point Energies=

$-114.427067$

Sum of electronic and thermal Enthalpies=

$-114.423256$

Sum of electronic and thermal Free Energies=

$-114.448074$

Total free energy in solution:

with all non electrostatic terms

$(\mathrm{a} \cdot \mathrm{u})=$.

Optimized geometry, SCF energy, three lower frequencies, thermochemistry and PCM energy for allyltrimethyltin.

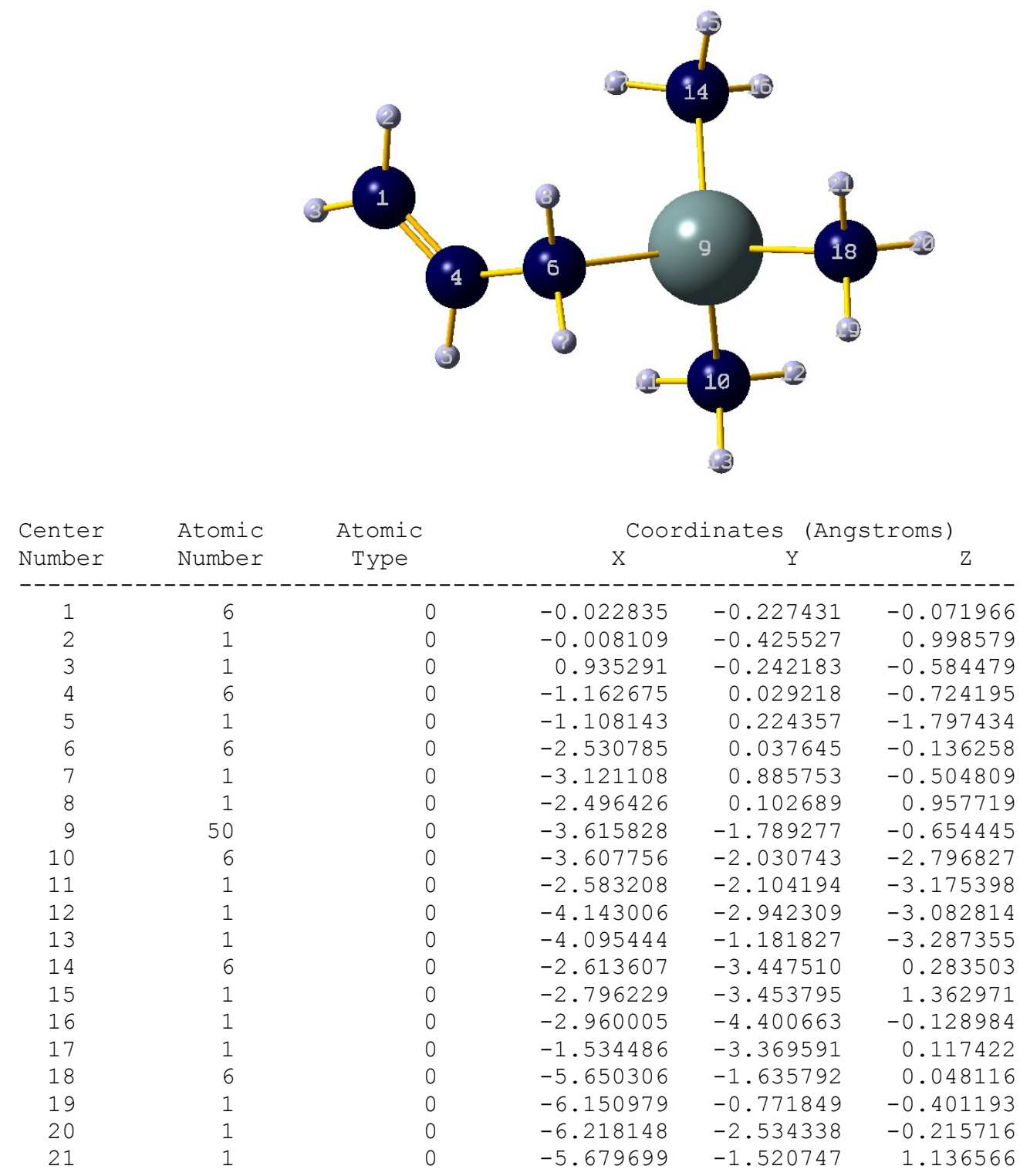

$\mathrm{HF}=-240.3469727$






\begin{tabular}{|c|c|c|c|c|c|c|c|c|c|c|}
\hline \multirow{2}{*}{\multicolumn{2}{|c|}{ Red. masses }} & $s--$ & \multicolumn{2}{|c|}{2.7468} & \multicolumn{3}{|c|}{2.0389} & \multicolumn{3}{|c|}{1.1667} \\
\hline & & -- & 0.002 & & & 0.004 & & & 0.003 & \\
\hline IR In & nten & -- & 0.084 & & & $0.20 \varepsilon$ & & & 0.044 & \\
\hline Atom & AN & $\mathrm{X}$ & $\mathrm{Y}$ & Z & $\mathrm{X}$ & $\mathrm{Y}$ & Z & $\mathrm{x}$ & $\mathrm{Y}$ & Z \\
\hline 1 & 6 & -0.02 & 0.01 & 0.24 & 0.05 & 0.22 & 0.03 & 0.02 & 0.07 & 0.02 \\
\hline 2 & 1 & -0.17 & 0.16 & 0.27 & 0.07 & 0.31 & 0.04 & 0.03 & 0.10 & 0.02 \\
\hline 3 & 1 & 0.05 & -0.08 & 0.37 & 0.06 & 0.27 & 0.03 & 0.03 & 0.09 & 0.02 \\
\hline 4 & 6 & 0.07 & -0.07 & 0.05 & 0.03 & 0.04 & 0.00 & 0.02 & 0.01 & 0.00 \\
\hline 5 & 1 & 0.22 & -0.22 & 0.02 & 0.01 & -0.05 & -0.01 & 0.01 & -0.02 & 0.00 \\
\hline 6 & 6 & -0.01 & 0.04 & -0.14 & 0.03 & -0.04 & 0.00 & 0.01 & -0.02 & 0.00 \\
\hline 7 & 1 & 0.06 & 0.00 & -0.32 & 0.00 & -0.05 & 0.01 & 0.01 & -0.02 & 0.00 \\
\hline 8 & 1 & -0.15 & 0.18 & -0.14 & 0.03 & -0.03 & 0.00 & 0.01 & -0.02 & 0.00 \\
\hline 9 & 50 & 0.00 & 0.00 & -0.02 & 0.00 & -0.02 & 0.00 & 0.00 & -0.01 & 0.00 \\
\hline 10 & 6 & -0.17 & -0.06 & -0.02 & -0.03 & -0.01 & 0.00 & -0.01 & -0.01 & 0.00 \\
\hline 11 & 1 & -0.20 & -0.10 & -0.10 & -0.05 & -0.41 & 0.02 & 0.00 & 0.53 & -0.07 \\
\hline 12 & 1 & -0.21 & -0.06 & 0.05 & -0.38 & 0.20 & -0.02 & 0.45 & -0.28 & 0.03 \\
\hline 13 & 1 & -0.19 & -0.07 & -0.01 & 0.31 & 0.18 & -0.01 & -0.48 & -0.26 & 0.03 \\
\hline 14 & 6 & 0.10 & 0.05 & -0.05 & -0.10 & -0.11 & -0.03 & -0.05 & -0.04 & -0.01 \\
\hline 15 & 1 & 0.12 & 0.04 & -0.05 & 0.00 & -0.04 & -0.01 & 0.06 & 0.04 & 0.01 \\
\hline 16 & 1 & 0.14 & 0.03 & -0.05 & -0.28 & -0.08 & 0.04 & -0.18 & -0.03 & 0.08 \\
\hline 17 & 1 & 0.09 & 0.11 & -0.07 & -0.11 & -0.26 & -0.14 & -0.06 & -0.16 & -0.12 \\
\hline 18 & 6 & 0.05 & 0.00 & 0.12 & 0.02 & 0.09 & 0.01 & 0.01 & 0.05 & 0.01 \\
\hline 19 & 1 & 0.02 & 0.01 & 0.18 & 0.08 & 0.14 & 0.03 & 0.04 & 0.08 & 0.04 \\
\hline 20 & 1 & 0.03 & 0.00 & 0.15 & -0.05 & 0.14 & -0.01 & -0.03 & 0.08 & -0.01 \\
\hline 21 & 1 & 0.13 & -0.02 & 0.13 & 0.03 & 0.07 & 0.01 & 0.02 & 0.02 & 0.01 \\
\hline
\end{tabular}

thermodynamics:

Sum of electronic and zero-point Energies=

$-240.168105$

Sum of electronic and thermal Enthalpies=

$-240.154175$

Sum of electronic and thermal Free Energies=

$-240.209027$

Total free energy in solution:

with all non electrostatic terms

$($ a.u. $)=-240.330308$

Optimized geometry, SCF energy, three lower frequencies, thermochemistry and PCM energy for $\mathbf{T S}_{\boldsymbol{\alpha}}$.



\begin{tabular}{cccrrr}
$\begin{array}{c}\text { Center } \\
\text { Number }\end{array}$ & $\begin{array}{c}\text { Atomic } \\
\text { Number }\end{array}$ & $\begin{array}{c}\text { Atomic } \\
\text { Type }\end{array}$ & \multicolumn{2}{c}{ Coordinates (Angstroms) } \\
-1 & $\mathbf{X}$ & $\mathrm{Y}$ & $\mathrm{Z}$ \\
\hline 1 & 6 & 0 & 3.988629 & 0.399040 & 0.397987 \\
2 & 1 & 0 & 3.748419 & 0.682994 & 1.421359 \\
3 & 1 & 0 & 5.026545 & 0.154173 & 0.190272 \\
4 & 6 & 0 & 3.057213 & 0.382517 & -0.565346 \\
5 & 1 & 0 & 3.358303 & 0.098348 & -1.575277 \\
6 & 6 & 0 & 1.615708 & 0.667376 & -0.375589 \\
7 & 1 & 0 & 1.225872 & 1.157739 & -1.277085 \\
8 & 1 & 0 & 1.490357 & 1.337641 & 0.483014 \\
9 & 50 & 0 & -0.817994 & 0.084277 & 0.026408 \\
10 & 6 & 0 & -1.857247 & -0.604990 & -1.714433 \\
11 & 1 & 0 & -1.169783 & -0.635509 & -2.565353
\end{tabular}




\begin{tabular}{|c|c|c|c|c|c|}
\hline 12 & 1 & 0 & -2.236921 & -1.615477 & -1.547319 \\
\hline 13 & 1 & 0 & -2.681679 & 0.074356 & -1.954363 \\
\hline 14 & 6 & 0 & -1.343230 & -0.461282 & 2.032649 \\
\hline 15 & 1 & 0 & -0.451024 & -0.448678 & 2.667538 \\
\hline 16 & 1 & 0 & -2.067931 & 0.247481 & 2.446159 \\
\hline 17 & 1 & 0 & -1.756669 & -1.472667 & 2.046530 \\
\hline 18 & 6 & 0 & -1.058146 & 2.251838 & -0.015301 \\
\hline 19 & 1 & 0 & -0.732615 & 2.690427 & -0.963607 \\
\hline 20 & 1 & 0 & -2.129185 & 2.460083 & 0.105874 \\
\hline 21 & 1 & 0 & -0.523275 & 2.745586 & 0.802104 \\
\hline 22 & 1 & 0 & 2.139405 & -1.747804 & -0.659856 \\
\hline 23 & 6 & 0 & 1.345395 & -1.478182 & 0.052220 \\
\hline 24 & 8 & 0 & 0.178727 & -1.955476 & -0.10863 \\
\hline 25 & 1 & 0 & 1.740141 & -1.236646 & 1.0555 \\
\hline
\end{tabular}



thermodynamics:

Sum of electronic and zero-point Energies=

Sum of electronic and thermal Enthalpies=

$-354.529961$

Sum of electronic and thermal Free Energies=

$-354.513920$

Total free energy in solution:

with all non electrostatic terms

$-354.572813$

$($ a.u. $)=-354.724733$

\section{Optimized geometry, SCF energy, three lower frequencies, thermochemistry and PCM energy for $\mathbf{T} \mathbf{S}_{\gamma}$.}




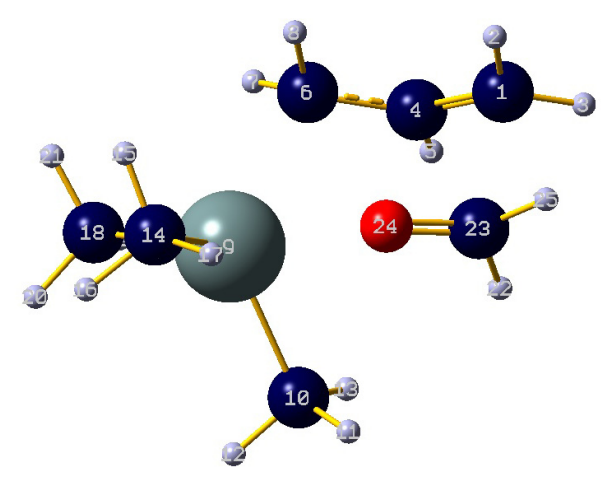

\begin{tabular}{|c|c|c|c|c|c|}
\hline \multirow{2}{*}{$\begin{array}{l}\text { Center } \\
\text { Number }\end{array}$} & \multirow{2}{*}{$\begin{array}{l}\text { Atomic } \\
\text { Number }\end{array}$} & \multirow{2}{*}{$\begin{array}{l}\text { Atomic } \\
\text { Type }\end{array}$} & \multicolumn{3}{|c|}{ Coordinates (Angstroms) } \\
\hline & & & $\mathrm{x}$ & Y & Z \\
\hline 1 & 6 & 0 & -3.171245 & 0.605570 & -0.252747 \\
\hline 2 & 1 & 0 & -3.316010 & 0.307974 & -1.288453 \\
\hline 3 & 1 & 0 & -4.061011 & 0.609214 & 0.372520 \\
\hline 4 & 6 & 0 & -2.091200 & 1.391860 & 0.078420 \\
\hline 5 & 1 & 0 & -2.084961 & 1.852116 & 1.068966 \\
\hline 6 & 6 & 0 & -0.892911 & 1.499975 & -0.691351 \\
\hline 7 & 1 & 0 & -0.318039 & 2.409415 & -0.509275 \\
\hline 8 & 1 & 0 & -1.007066 & 1.293127 & -1.757892 \\
\hline 9 & 50 & 0 & 0.714307 & -0.029229 & 0.008213 \\
\hline 10 & 6 & 0 & 0.586239 & -0.717524 & 2.049946 \\
\hline 11 & 1 & 0 & 0.159979 & -1.722873 & 2.106012 \\
\hline 12 & 1 & 0 & 1.591267 & -0.734215 & 2.484904 \\
\hline 13 & 1 & 0 & -0.025227 & -0.043654 & 2.660182 \\
\hline 14 & 6 & 0 & 1.553128 & -1.334864 & -1.480098 \\
\hline 15 & 1 & 0 & 1.537999 & -0.848778 & -2.461733 \\
\hline 16 & 1 & 0 & 2.597170 & -1.556000 & -1.232989 \\
\hline 17 & 1 & 0 & 0.985952 & -2.265836 & -1.541739 \\
\hline 18 & 6 & 0 & 2.228959 & 1.546440 & 0.159939 \\
\hline 19 & 1 & 0 & 1.952261 & 2.297569 & 0.909586 \\
\hline 20 & 1 & 0 & 3.193854 & 1.116898 & 0.455858 \\
\hline 21 & 1 & 0 & 2.370147 & 2.058056 & -0.799485 \\
\hline 22 & 1 & 0 & -2.254887 & -1.064036 & 1.358352 \\
\hline 23 & 6 & 0 & -2.265381 & -1.236986 & 0.270503 \\
\hline 24 & 8 & 0 & -1.192930 & -1.381873 & -0.366875 \\
\hline 25 & 1 & 0 & -3.178836 & -1.719363 & -0.108122 \\
\hline
\end{tabular}

\begin{tabular}{|c|c|c|c|c|c|c|c|c|c|c|}
\hline \multicolumn{11}{|c|}{2} \\
\hline \multirow{2}{*}{\multicolumn{2}{|c|}{ Frequencies }} & \multicolumn{3}{|c|}{${ }_{\mathrm{A}}^{1}$} & \multirow{2}{*}{\multicolumn{3}{|c|}{$\begin{array}{c}A \\
47.5780\end{array}$}} & \multicolumn{3}{|c|}{ A } \\
\hline & & \multicolumn{3}{|c|}{-269.9856} & & & & \multicolumn{3}{|c|}{64.7837} \\
\hline \multicolumn{2}{|c|}{ Red. masses } & $s--$ & \multicolumn{2}{|c|}{9.1326} & \multicolumn{3}{|c|}{1.1075} & \multicolumn{3}{|c|}{3.1852} \\
\hline \multicolumn{2}{|c|}{ Frc consts } & -- & \multicolumn{2}{|c|}{0.3922} & \multicolumn{3}{|c|}{0.0015} & \multicolumn{3}{|c|}{0.0079} \\
\hline \multicolumn{2}{|c|}{ IR Inten } & -- & \multicolumn{2}{|c|}{70.8448} & \multicolumn{3}{|c|}{0.0324} & \multicolumn{3}{|c|}{0.4204} \\
\hline Atom & AN & $\mathrm{X}$ & $\mathrm{Y}$ & Z & $\mathrm{x}$ & $\mathrm{Y}$ & Z & $\mathrm{X}$ & $\mathrm{Y}$ & Z \\
\hline 1 & 6 & -0.27 & 0.48 & -0.11 & 0.00 & 0.00 & 0.01 & 0.01 & 0.02 & 0.19 \\
\hline 2 & 1 & -0.14 & 0.09 & -0.02 & -0.01 & -0.02 & 0.02 & -0.11 & 0.13 & 0.17 \\
\hline 3 & 1 & -0.22 & 0.35 & -0.05 & 0.01 & 0.02 & 0.02 & 0.07 & -0.03 & 0.28 \\
\hline 4 & 6 & -0.07 & -0.03 & 0.02 & 0.01 & 0.01 & -0.02 & 0.05 & -0.02 & 0.14 \\
\hline 5 & 1 & -0.01 & -0.08 & 0.05 & 0.02 & 0.03 & -0.03 & 0.16 & -0.09 & 0.18 \\
\hline 6 & 6 & 0.09 & -0.22 & 0.05 & 0.00 & -0.02 & -0.04 & -0.02 & 0.01 & 0.03 \\
\hline 7 & 1 & -0.07 & -0.08 & -0.07 & 0.00 & -0.01 & -0.07 & 0.00 & 0.00 & 0.01 \\
\hline 8 & 1 & -0.05 & -0.07 & 0.03 & -0.01 & -0.05 & -0.03 & -0.12 & 0.05 & 0.04 \\
\hline 9 & 50 & 0.03 & 0.06 & -0.01 & 0.00 & 0.00 & 0.00 & -0.01 & 0.00 & -0.02 \\
\hline 10 & 6 & -0.04 & -0.03 & -0.03 & 0.01 & -0.02 & -0.01 & -0.14 & 0.12 & 0.01 \\
\hline 11 & 1 & -0.02 & -0.03 & 0.00 & 0.03 & -0.02 & -0.01 & -0.24 & 0.17 & 0.07 \\
\hline 12 & 1 & -0.03 & -0.02 & -0.04 & 0.01 & 0.00 & -0.01 & -0.15 & 0.04 & 0.04 \\
\hline 13 & 1 & -0.03 & -0.03 & -0.02 & 0.00 & -0.03 & 0.00 & -0.07 & 0.23 & -0.04 \\
\hline 14 & 6 & -0.03 & -0.01 & 0.01 & 0.02 & 0.03 & -0.02 & 0.12 & -0.11 & 0.14 \\
\hline 15 & 1 & -0.02 & -0.01 & 0.01 & 0.55 & 0.27 & 0.09 & 0.35 & -0.13 & 0.13 \\
\hline
\end{tabular}




$\begin{array}{rrrrrrrrrrr}16 & 1 & -0.03 & -0.01 & 0.01 & -0.14 & -0.40 & 0.30 & 0.05 & -0.20 & 0.35 \\ 17 & 1 & -0.01 & -0.02 & 0.00 & -0.28 & 0.24 & -0.42 & 0.04 & -0.06 & 0.06 \\ 18 & 6 & 0.09 & -0.03 & 0.04 & -0.02 & 0.01 & 0.03 & 0.00 & 0.00 & -0.05 \\ 19 & 1 & 0.07 & -0.02 & 0.02 & -0.03 & -0.01 & 0.04 & 0.00 & 0.03 & -0.09 \\ 20 & 1 & 0.12 & 0.04 & 0.03 & -0.01 & 0.02 & 0.03 & 0.00 & 0.00 & -0.02 \\ 21 & 1 & 0.06 & -0.03 & 0.03 & -0.01 & 0.03 & 0.04 & 0.02 & -0.04 & -0.07 \\ 22 & 1 & 0.14 & -0.02 & 0.04 & 0.01 & 0.03 & 0.04 & 0.06 & -0.20 & -0.08 \\ 23 & 6 & 0.09 & -0.41 & 0.11 & 0.00 & 0.01 & 0.04 & 0.03 & -0.06 & -0.10 \\ 24 & 8 & -0.12 & -0.25 & -0.03 & -0.01 & -0.01 & 0.03 & 0.01 & 0.02 & -0.15 \\ 25 & 1 & -0.16 & 0.10 & 0.07 & -0.01 & 0.00 & 0.06 & 0.02 & -0.01 & -0.14\end{array}$

thermodynamics:

Sum of electronic and zero-point Energies=

$-354.581671$

Sum of electronic and thermal Enthalpies=

$-354.566198$

Sum of electronic and thermal Free Energies=

$-354.623274$

Total free energy in solution:

with all non electrostatic terms

$(\mathrm{a} . \mathrm{u})=.\quad-354.778745$

Optimized geometry, SCF energy, three lower frequencies, thermochemistry and PCM energy for product.



\begin{tabular}{|c|c|c|c|c|c|}
\hline \multirow{2}{*}{$\begin{array}{l}\text { Center } \\
\text { Number }\end{array}$} & \multirow{2}{*}{$\begin{array}{l}\text { Atomic } \\
\text { Number }\end{array}$} & \multirow{2}{*}{$\begin{array}{l}\text { Atomic } \\
\text { Type }\end{array}$} & \multicolumn{3}{|c|}{ Coordinates (Angstroms) } \\
\hline & & & $\mathrm{x}$ & $\mathrm{Y}$ & Z \\
\hline 1 & 6 & 0 & 0.358230 & -0.057870 & -0.162543 \\
\hline 2 & 1 & 0 & 1.073947 & 0.394642 & 0.538743 \\
\hline 3 & 1 & 0 & 0.945159 & -0.590087 & -0.920766 \\
\hline 4 & 6 & 0 & -0.488300 & 1.003720 & -0.798193 \\
\hline 5 & 1 & 0 & -1.053774 & 1.639277 & -0.113011 \\
\hline 6 & 6 & 0 & -0.607800 & 1.210742 & -2.110242 \\
\hline 7 & 1 & 0 & -0.065294 & 0.605176 & -2.834443 \\
\hline 8 & 1 & 0 & -1.244454 & 1.995559 & -2.511451 \\
\hline 9 & 8 & 0 & 0.403060 & -1.989835 & 1.248518 \\
\hline 10 & 6 & 0 & -0.468479 & -1.078740 & 0.624707 \\
\hline 11 & 1 & 0 & -1.162691 & -1.582726 & -0.069722 \\
\hline 12 & 1 & 0 & -1.092202 & -0.544011 & 1.363886 \\
\hline 13 & 50 & 0 & -0.210019 & -3.526699 & 2.295190 \\
\hline 14 & 6 & 0 & 1.619863 & -4.513581 & 2.801688 \\
\hline 15 & 1 & 0 & 1.440587 & -5.334003 & 3.504177 \\
\hline 16 & 1 & 0 & 2.094131 & -4.921929 & 1.904255 \\
\hline 17 & 1 & 0 & 2.312016 & -3.804325 & 3.265473 \\
\hline 18 & 6 & 0 & -1.266079 & -2.810122 & 4.025717 \\
\hline 19 & 1 & 0 & -0.638066 & -2.125663 & 4.604163 \\
\hline 20 & 1 & 0 & -2.180084 & -2.282520 & 3.735428 \\
\hline 21 & 1 & 0 & -1.547111 & -3.649673 & 4.670838 \\
\hline 22 & 6 & 0 & -1.500566 & -4.738113 & 1.075033 \\
\hline 23 & 1 & 0 & -0.994470 & -5.034873 & 0.151308 \\
\hline 24 & 1 & 0 & -1.786872 & -5.645064 & 1.618596 \\
\hline
\end{tabular}


$\mathrm{HF}=-354.8520877$

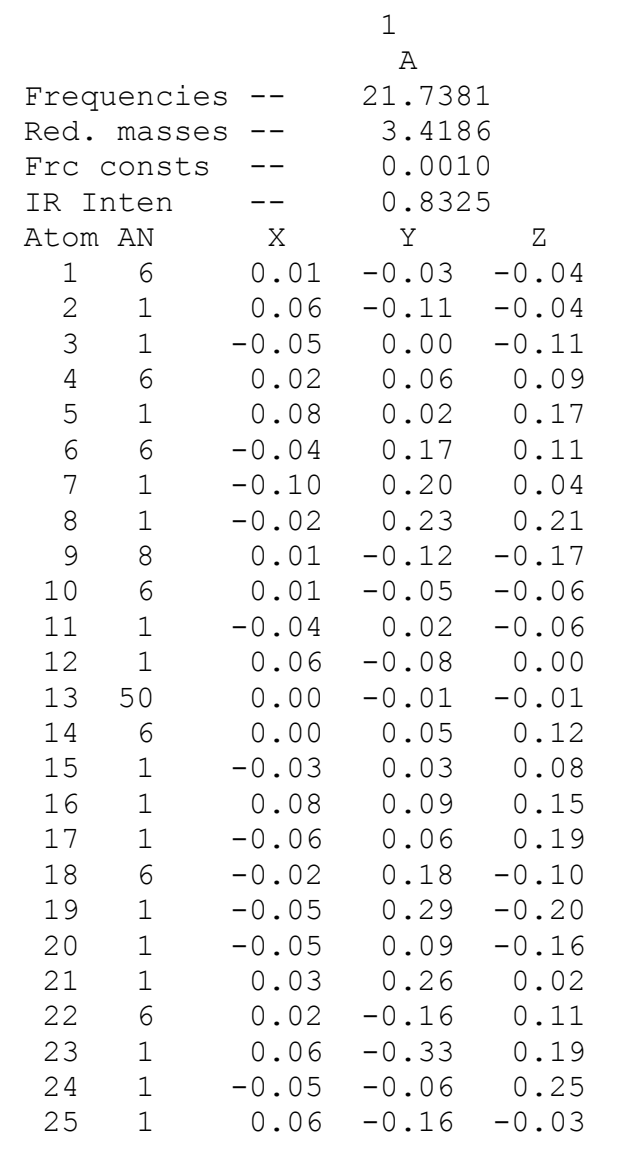

\begin{tabular}{rrr}
\multicolumn{3}{c}{ I } \\
\multicolumn{3}{c}{37.0762} \\
\multicolumn{3}{c}{2.0173} \\
\multicolumn{3}{c}{0.0016} \\
X & \multicolumn{1}{c}{$\mathrm{Y}$} \\
-0.06 & \multicolumn{1}{c}{0.06} & \multicolumn{1}{c}{0.07} \\
-0.21 & 0.11 & 0.20 \\
0.12 & 0.15 & 0.14 \\
-0.07 & -0.01 & -0.02 \\
-0.30 & -0.14 & -0.09 \\
0.18 & 0.08 & -0.03 \\
0.41 & 0.20 & 0.04 \\
0.16 & 0.02 & -0.10 \\
-0.05 & -0.01 & -0.02 \\
-0.06 & -0.06 & -0.09 \\
0.08 & -0.10 & -0.20 \\
-0.21 & -0.16 & -0.14 \\
0.00 & -0.01 & 0.00 \\
0.03 & 0.02 & -0.06 \\
0.10 & 0.16 & 0.12 \\
-0.10 & -0.16 & -0.04 \\
0.11 & 0.09 & -0.28 \\
0.06 & 0.01 & 0.04 \\
0.08 & 0.03 & 0.00 \\
0.04 & -0.01 & 0.07 \\
0.10 & 0.01 & 0.06 \\
-0.04 & -0.03 & 0.07 \\
-0.06 & -0.07 & 0.07 \\
-0.04 & -0.01 & 0.11 \\
-0.03 & -0.03 & 0.06
\end{tabular}

\begin{tabular}{rrr}
\multicolumn{3}{c}{3} \\
\multicolumn{3}{c}{ A } \\
\multicolumn{3}{c}{1.8092} \\
\multicolumn{3}{c}{0.0695} \\
\multicolumn{3}{c}{0.0028} \\
\multicolumn{1}{c}{$\mathrm{X}$} & \multicolumn{1}{c}{$\mathrm{Y}$} & \multicolumn{1}{c}{$\mathrm{Z}$} \\
-0.02 & 0.02 & \multicolumn{1}{c}{0.02} \\
-0.06 & 0.04 & 0.05 \\
0.03 & 0.04 & 0.04 \\
-0.03 & -0.01 & -0.01 \\
-0.09 & -0.04 & -0.03 \\
0.03 & 0.00 & -0.02 \\
0.10 & 0.03 & 0.01 \\
0.02 & -0.02 & -0.04 \\
0.00 & 0.00 & 0.00 \\
-0.01 & -0.01 & -0.02 \\
0.02 & -0.03 & -0.04 \\
-0.04 & -0.04 & -0.03 \\
0.00 & 0.00 & 0.00 \\
-0.01 & -0.02 & -0.01 \\
-0.09 & -0.38 & -0.45 \\
0.25 & 0.46 & -0.10 \\
-0.19 & -0.18 & 0.49 \\
0.02 & 0.01 & 0.01 \\
0.02 & 0.02 & -0.01 \\
0.01 & -0.01 & 0.02 \\
0.04 & 0.01 & 0.03 \\
-0.01 & 0.01 & 0.01 \\
-0.01 & -0.02 & 0.02 \\
-0.03 & 0.02 & 0.03 \\
0.00 & 0.02 & 0.00 \\
& &
\end{tabular}

thermodynamics:

Sum of electronic and zero-point Energies=

$-354.639469$

Sum of electronic and thermal Enthalpies=

$-354.623004$

Sum of electronic and thermal Free Energies=

$-354.685549$

Total free energy in solution:

with all non electrostatic terms (a.u.) $=\quad-354.835389$

\section{With bromobenzaldehyde}

Optimized geometry, SCF energy, three lower frequencies, thermochemistry and PCM energy for bromobenzaldehyde.

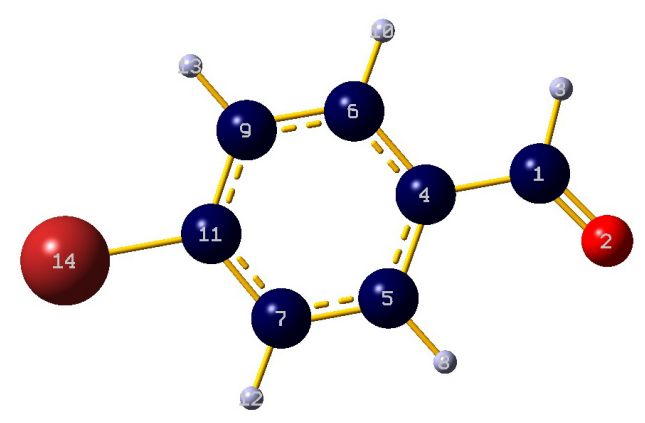




\begin{tabular}{cccrrr}
$\begin{array}{c}\text { Center } \\
\text { Number }\end{array}$ & $\begin{array}{c}\text { Atomic } \\
\text { Number }\end{array}$ & $\begin{array}{c}\text { Atomic } \\
\text { Type }\end{array}$ & \multicolumn{2}{c}{ Coordinates (Angstroms) } \\
-1 & 6 & 0 & -.048739 & -.000043 & -.021837 \\
1 & 8 & 0 & -.002325 & .015356 & 1.191061 \\
2 & 1 & 0 & .884481 & -.015862 & -.628266 \\
3 & 6 & 0 & -1.297689 & .000048 & -.812251 \\
4 & 6 & 0 & -2.540940 & .019149 & -.166581 \\
5 & 6 & 0 & -1.242960 & -.019110 & -2.209517 \\
6 & 6 & 0 & -3.715362 & .019208 & -.905980 \\
7 & 1 & 0 & -2.563826 & .033765 & .919472 \\
8 & 6 & 0 & -2.412096 & -.019281 & -2.963664 \\
9 & 1 & 0 & -.277610 & -.034091 & -2.712142 \\
10 & 6 & 0 & -3.638363 & -.000090 & -2.300966 \\
11 & 1 & 0 & -4.683463 & .033969 & -.416280 \\
12 & 1 & 0 & -2.378848 & -.034012 & -4.047854 \\
13 & 35 & 0 & -5.236216 & -.000182 & -3.314873 \\
14 & & 0 & & &
\end{tabular}

\begin{tabular}{|c|c|c|c|c|c|c|c|c|c|}
\hline & \multicolumn{3}{|c|}{1} & \multicolumn{3}{|c|}{2} & \multicolumn{3}{|c|}{3} \\
\hline & & A & & & A & & & A & \\
\hline Frequencies & -- & 80.48 & & & 52.86 & & & 69.356 & \\
\hline Red. masses & -- & 9.73 & & & 9.69 & & & 3.475 & \\
\hline Frc consts & -- & .03 & & & .15 & & & .058 & \\
\hline IR Inten & -- & 2.43 & & & 5.44 & & & 6.696 & \\
\hline Atom AN & $\mathrm{X}$ & $\mathrm{Y}$ & Z & $\mathrm{X}$ & $\mathrm{Y}$ & Z & $\mathrm{X}$ & $\mathrm{Y}$ & Z \\
\hline 6 & .00 & .12 & .00 & .07 & .00 & -.10 & .00 & .27 & .00 \\
\hline 2 & .00 & .46 & -.01 & .49 & -.01 & -.12 & .00 & -.19 & .00 \\
\hline 3 & .00 & .08 & .00 & -.13 & .01 & -.41 & .01 & .82 & -.01 \\
\hline 4 & .00 & -.19 & .00 & -.12 & .00 & .18 & .00 & .12 & .00 \\
\hline 5 & .00 & -.35 & .00 & -.13 & .00 & .19 & .00 & .04 & .00 \\
\hline 6 & .00 & -.17 & .00 & -.15 & .00 & .19 & .00 & -.02 & .00 \\
\hline 7 & .00 & -.37 & .01 & -.15 & .00 & .17 & .00 & -.07 & .00 \\
\hline 8 & .00 & -.39 & .01 & -.16 & .01 & .19 & .00 & .11 & .00 \\
\hline 6 & .00 & -.16 & .00 & -.13 & .00 & .19 & .00 & -.21 & .00 \\
\hline 10 & .00 & -.08 & .00 & -.15 & .00 & .19 & .00 & .00 & .00 \\
\hline 11 & .00 & -.20 & .00 & -.14 & .00 & .16 & .00 & -.15 & .00 \\
\hline 12 & .00 & -.45 & .01 & -.14 & .00 & .17 & .00 & -.04 & .00 \\
\hline 13 & .00 & -.06 & .00 & -.12 & .00 & .19 & .00 & -.33 & .00 \\
\hline 35 & .00 & .12 & .00 & .02 & .00 & -.13 & .00 & .03 & .00 \\
\hline
\end{tabular}

thermodynamics:

Sum of electronic and zero-point Energies=

Sum of electronic and thermal Enthalpies=

$-2916.111521$

Sum of electronic and thermal Free Energies=

$-2916.153957$

Total free energy in solution:

with all non electrostatic terms

$(a \cdot u)=$.

Optimized geometry, SCF energy, three lower frequencies, thermochemistry and PCM energy for $\mathbf{T S}_{\boldsymbol{\gamma}}$.

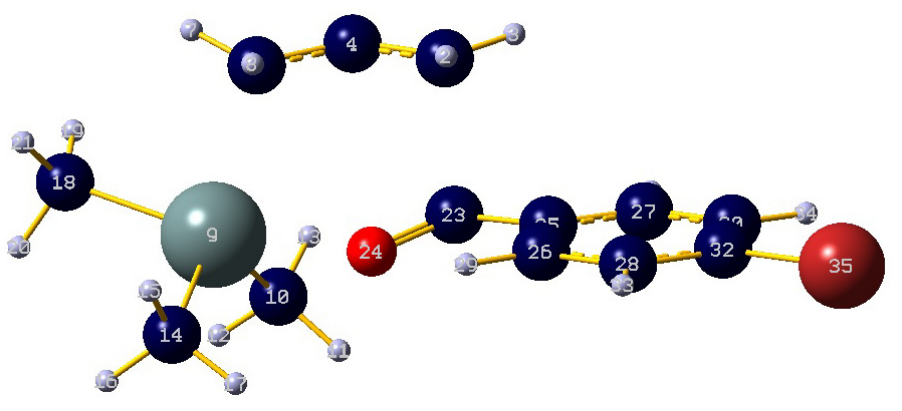




\begin{tabular}{|c|c|c|c|c|c|}
\hline \multirow{2}{*}{$\begin{array}{l}\text { Center } \\
\text { Number }\end{array}$} & \multirow{2}{*}{$\begin{array}{l}\text { Atomic } \\
\text { Number }\end{array}$} & \multirow{2}{*}{$\begin{array}{l}\text { Atomic } \\
\text { Type }\end{array}$} & \multicolumn{3}{|c|}{ Coordinates (Angstroms) } \\
\hline & & & $\mathrm{X}$ & Y & Z \\
\hline 1 & 6 & 0 & .375690 & 2.047375 & -.932332 \\
\hline 2 & 1 & 0 & -.088971 & 1.470180 & -1.727549 \\
\hline 3 & 1 & 0 & -.239434 & 2.840843 & -.516017 \\
\hline 4 & 6 & 0 & 1.754859 & 2.187874 & -.936215 \\
\hline 5 & 1 & 0 & 2.189043 & 2.970019 & -.309420 \\
\hline 6 & 6 & 0 & 2.652313 & 1.269663 & -1.532048 \\
\hline 7 & 1 & 0 & 3.658231 & 1.641213 & -1.724294 \\
\hline 8 & 1 & 0 & 2.253148 & .663651 & -2.347398 \\
\hline 9 & 50 & 0 & 3.258502 & -.343630 & .092129 \\
\hline 10 & 6 & 0 & 3.173822 & .282725 & 2.154356 \\
\hline 11 & 1 & 0 & 2.313670 & -.155021 & 2.668365 \\
\hline 12 & 1 & 0 & 4.090574 & -.034000 & 2.663059 \\
\hline 13 & 1 & 0 & 3.112208 & 1.373601 & 2.235447 \\
\hline 14 & 6 & 0 & 3.014816 & -2.422205 & -.401343 \\
\hline 15 & 1 & 0 & 2.948448 & -2.548011 & -1.487503 \\
\hline 16 & 1 & 0 & 3.881315 & -2.992276 & -.049658 \\
\hline 17 & 1 & 0 & 2.104369 & -2.821883 & .050233 \\
\hline 18 & 6 & 0 & 5.370463 & .005149 & -.391426 \\
\hline 19 & 1 & 0 & 5.665626 & 1.037013 & -.166782 \\
\hline 20 & 1 & 0 & 6.002233 & -.664042 & .205635 \\
\hline 21 & 1 & 0 & 5.579258 & -.189512 & -1.449869 \\
\hline 22 & 1 & 0 & .450644 & 1.172762 & 1.334281 \\
\hline 23 & 6 & 0 & .179424 & .535953 & .476288 \\
\hline 24 & 8 & 0 & .959079 & -.413428 & .144072 \\
\hline 25 & 6 & 0 & -1.293689 & .343250 & .314466 \\
\hline 26 & 6 & 0 & -1.784805 & -.709109 & -.465523 \\
\hline 27 & 6 & 0 & -2.198695 & 1.178271 & .977935 \\
\hline 28 & 6 & 0 & -3.153753 & -.917027 & -.595151 \\
\hline 29 & 1 & 0 & -1.076320 & -1.369724 & -.956202 \\
\hline 30 & 6 & 0 & -3.571066 & .980769 & .859713 \\
\hline 31 & 1 & 0 & -1.828591 & 1.991289 & 1.599934 \\
\hline 32 & 6 & 0 & -4.037542 & -.066616 & .067898 \\
\hline 33 & 1 & 0 & -3.535413 & -1.735030 & -1.197547 \\
\hline 34 & 1 & 0 & -4.272135 & 1.627252 & 1.377205 \\
\hline 35 & 35 & 0 & -5.905618 & -.345309 & -.101162 \\
\hline
\end{tabular}

\begin{tabular}{|c|c|c|c|c|}
\hline 2 & & & 3 & \\
\hline A & & & A & \\
\hline 29.789 & & & 39.431 & \\
\hline 4.256 & & & 4.674 & \\
\hline .002 & & & .004 & \\
\hline .043 & & & .277 & \\
\hline $\mathrm{Y}$ & Z & $\mathrm{X}$ & Y & Z \\
\hline-.03 & -.13 & -.06 & -.10 & .04 \\
\hline-.05 & -.15 & -.09 & -.09 & .05 \\
\hline-.04 & -.18 & -.04 & -.11 & .08 \\
\hline .00 & -.06 & -.06 & -.09 & -.04 \\
\hline .02 & -.04 & -.02 & -.08 & -.07 \\
\hline .02 & .00 & -.09 & -.09 & -.08 \\
\hline .04 & .07 & -.10 & -.10 & -.16 \\
\hline .02 & -.03 & -.14 & -.13 & -.03 \\
\hline .00 & .02 & .02 & .02 & -.01 \\
\hline-.13 & .05 & -.06 & .07 & -.02 \\
\hline-.21 & -.04 & -.04 & .04 & -.02 \\
\hline-.12 & .10 & -.05 & .11 & .00 \\
\hline-.14 & .13 & -.10 & .06 & -.04 \\
\hline .03 & -.11 & .19 & -.02 & .07 \\
\hline .09 & -.12 & .31 & -.08 & .07 \\
\hline .03 & -.16 & .19 & .06 & .19 \\
\hline-.03 & -.13 & .17 & -.05 & .00 \\
\hline .08 & .23 & .00 & .20 & .04 \\
\hline .09 & .28 & -.11 & .24 & -.01 \\
\hline
\end{tabular}




\begin{tabular}{|c|c|c|c|c|c|c|c|c|c|c|}
\hline 20 & 1 & .17 & -.01 & .01 & .00 & .09 & .27 & .05 & .29 & .10 \\
\hline 21 & 1 & .03 & -.03 & .05 & .14 & .12 & .24 & .05 & .16 & .06 \\
\hline 22 & 1 & .06 & -.01 & .00 & .01 & -.02 & -.13 & .03 & -.16 & 02 \\
\hline 23 & 6 & -.12 & -.37 & .34 & .01 & -.01 & -.13 & .01 & -.13 & 0 \\
\hline 24 & 8 & -.15 & -.09 & .08 & .00 & -.02 & -.13 & .02 & -.12 & -.04 \\
\hline 25 & 6 & -.04 & -.03 & .04 & .00 & .01 & -.11 & .01 & -.13 & 04 \\
\hline 26 & 6 & -.03 & -.02 & .00 & -.03 & .00 & -.09 & -.03 & -.06 & -.02 \\
\hline 27 & 6 & -.02 & .00 & .02 & .03 & .02 & -.10 & .04 & -.15 & .11 \\
\hline 28 & 6 & -.02 & .01 & -.02 & -.03 & .00 & -.04 & -.04 & -.01 & -.03 \\
\hline 29 & 1 & -.03 & -.02 & .01 & -.05 & -.01 & -.10 & -.05 & -.05 & -.07 \\
\hline 30 & 6 & -.02 & .01 & .00 & .02 & .02 & -.05 & .03 & -.09 & 10 \\
\hline 31 & 1 & -.01 & -.01 & .03 & .05 & .03 & -.12 & .07 & -.20 & 1 \\
\hline 32 & 6 & -.01 & .01 & -.01 & -.01 & .01 & -.01 & -.01 & -.02 & .0 \\
\hline 33 & 1 & -.03 & .02 & -.03 & -.06 & -.01 & -.01 & -.07 & .05 & -.09 \\
\hline 34 & 1 & -.02 & .02 & -.02 & .04 & .03 & -.03 & .05 & -.10 & 1 \\
\hline 35 & 35 & .00 & .00 & .00 & -.01 & .00 & .08 & -.02 & .09 & -.0 \\
\hline
\end{tabular}

thermodynamics:

Sum of electronic and zero-point Energies=

$-3156.267242$

Sum of electronic and thermal Enthalpies=

$-3156.245813$

Sum of electronic and thermal Free Energies=

$-3156.318717$

Total free energy in solution:

with all non electrostatic terms $\quad(a . u)=$.

Optimized geometry, SCF energy, three lower frequencies, thermochemistry and PCM energy for product.



\begin{tabular}{|c|c|c|c|c|c|}
\hline \multirow{2}{*}{$\begin{array}{l}\text { Center } \\
\text { Number }\end{array}$} & \multirow{2}{*}{$\begin{array}{l}\text { Atomic } \\
\text { Number }\end{array}$} & \multirow{2}{*}{$\begin{array}{c}\text { Atomic } \\
\text { Type }\end{array}$} & \multicolumn{3}{|c|}{ Coordinates (Angstroms) } \\
\hline & & & $\mathrm{X}$ & $\mathrm{Y}$ & $\mathrm{Z}$ \\
\hline 1 & 6 & 0 & -.101443 & -.040167 & .134324 \\
\hline 2 & 1 & 0 & -.284148 & .079897 & 1.211822 \\
\hline 3 & 1 & 0 & .974647 & -.206199 & .006170 \\
\hline 4 & 6 & 0 & -.533302 & 1.188172 & -.608472 \\
\hline 5 & 1 & 0 & -1.566034 & 1.505690 & -.459875 \\
\hline 6 & 6 & 0 & .248769 & 1.895706 & -1.425404 \\
\hline 7 & 1 & 0 & 1.287301 & 1.620401 & -1.602773 \\
\hline 8 & 1 & 0 & -.114292 & 2.781510 & -1.940903 \\
\hline 9 & 8 & 0 & -.235543 & -2.386804 & .433185 \\
\hline 10 & 6 & 0 & -.813943 & -1.338911 & -.306680 \\
\hline 11 & 1 & 0 & -.626366 & -1.461571 & -1.387606 \\
\hline 12 & 50 & 0 & -.395410 & -4.294910 & -.036342 \\
\hline 13 & 6 & 0 & 1.517979 & -5.078072 & .524826 \\
\hline 14 & 1 & 0 & 1.515482 & -6.172837 & .502914 \\
\hline 15 & 1 & 0 & 2.294674 & -4.718188 & -.156693 \\
\hline 16 & 1 & 0 & 1.771392 & -4.752937 & 1.538625 \\
\hline 17 & 6 & 0 & -1.980179 & -5.172466 & 1.114100 \\
\hline 18 & 1 & 0 & -1.803671 & -5.032410 & 2.185014 \\
\hline 19 & 1 & 0 & -2.939468 & -4.715099 & .855853 \\
\hline 20 & 1 & 0 & -2.040464 & -6.247690 & .912909 \\
\hline 21 & 6 & 0 & -.782240 & -4.446733 & -2.143539 \\
\hline 2 & 1 & 0 & -.000998 & -3.949679 & -2.726836 \\
\hline
\end{tabular}




$\begin{array}{ll}23 & 1 \\ 24 & 1 \\ 25 & 6 \\ 26 & 6 \\ 27 & 6 \\ 28 & 6 \\ 29 & 1 \\ 30 & 6 \\ 31 & 1 \\ 32 & 6 \\ 33 & 1 \\ 34 & 1 \\ 35 & 35\end{array}$

$\begin{array}{rrr}-.812001 & -5.501222 & -2.438370 \\ -1.747677 & -3.995060 & -2.390848 \\ -2.318920 & -1.258154 & -.107155 \\ -3.174572 & -.941104 & -1.166377 \\ -2.876980 & -1.480574 & 1.156837 \\ -4.552788 & -.842265 & -.978285 \\ -2.760828 & -.765295 & -2.157433 \\ -4.250896 & -1.389078 & 1.363418 \\ -2.221312 & -1.740076 & 1.983538 \\ -5.079212 & -1.068803 & .289633 \\ -5.209906 & -.598153 & -1.806597 \\ -4.677148 & -1.564615 & 2.345881 \\ -6.954996 & -.946824 & .559476\end{array}$

$\mathrm{HF}=-3156.6030498$

Frequencies -

Red. masses --

Frc consts --

$\begin{array}{ll}\text { IR Inten } & -- \\ \text { Atom AN } & \mathrm{X}\end{array}$

$1-6-.01$

$\begin{array}{lll}2 & 1 & .00\end{array}$

$\begin{array}{lll}3 & 1 & -.01\end{array}$

$\begin{array}{lll}4 & 6 & -.01\end{array}$

$\begin{array}{lll}5 & 1 & -.01\end{array}$

$\begin{array}{lll}6 & 6 & -.02\end{array}$

$\begin{array}{lll}7 & 1 & -.02\end{array}$

$\begin{array}{lll}8 & 1 & -.02 \\ 9 & 8 & -.01\end{array}$

$\begin{array}{lll}10 & 6 & -.01\end{array}$

$\begin{array}{lll}11 & 1 & -.02\end{array}$

$\begin{array}{lll}12 & 50 \quad .01\end{array}$

$136-.07$

$\begin{array}{lll}14 & 1 & -.08\end{array}$

$\begin{array}{lll}15 & 1 & .01\end{array}$

$\begin{array}{lll}16 & 1 & -.17\end{array}$

$\begin{array}{lll}17 & 6 & -.14\end{array}$

$181-.28$

$\begin{array}{lll}19 & 1 & -.11\end{array}$

$201-.11$

$\begin{array}{lll}21 & 6 & .25\end{array}$

$\begin{array}{lll}22 & 1 & .30\end{array}$

$\begin{array}{lll}23 & 1 & .33\end{array}$

$\begin{array}{lll}24 & 1 & .26\end{array}$

$\begin{array}{lll}25 & 6 & -.01\end{array}$

$26-6-.02$

$27 \quad 6 \quad .00$

$\begin{array}{lll}28 & 6 & -.02\end{array}$

$29-1 \quad-.03$

$\begin{array}{lll}30 & 6 & .00\end{array}$

$\begin{array}{lll}31 & 1 & .01\end{array}$

$\begin{array}{lll}32 & 6 & -.01\end{array}$

$33-1-.02$

$\begin{array}{lll}34 & 1 & .01\end{array}$

$\begin{array}{lll}35 & 35 & .00\end{array}$
1

A

11.1827

3.1380

.0002

.0065

Y Z

$.00-.03$

$.01-.03$

$.00-.04$

$-.01-.04$

$-.01-.03$

$-.02-.06$

$-.03-.07$

$-.03-.07$

.00

$-.01$

$-.02$

.00
-.02

$-.02$

$-.02$

$-.02$

.02

.03

.01

.01

.00

.04

.00

$-.05$

.00

$-.02$

.02

$-.02$

$-.03$

.02

.03

.00

$-.03$

.04

.01
2

22.0458

6.9366

.0020

.7361

$\mathrm{X}$

$-.08$

$-.08$

$\mathrm{Y}$

$-.05$

$-.03$

$-.05$

$-.06$

$-.05$

$-.10$

$-.11$

$-.10$

$-.04$

$-.06$

$-.08$

$-.04$

.10

.10

.16

.12

$-.20$

$-.36$

$-.15$

$-.17$

.00

.00

.10

.01

$-.08$

$-.05$

.03

$-.09$

.08

.06

$-.04$

$-.15$

.05

.15

$-.07$

.14
3

39.4033

4.2524

.0039

.2242

X

.03

$-.03$

.03

.16

.19

.26

.24

.35

$-.05$

$-.01$

$-.02$

$-.01$

.00

.02

$-.01$

.00

.00

.04

.01

$-.03$

$-.01$

$-.05$

.06

$-.04$

$-.01$

.00

$-.03$

.00

.01

$-.03$

$-.04$

$-.02$

.00

$-.04$

$-.03$
$\mathrm{Y}$

\section{Z}

$.00 \quad .01$

$.10-.01$

$\begin{array}{ll}-.08 \quad .09 \\ -.03 & -.12\end{array}$

$-.03-.12$

$.07-.19$

$-.19-.17$

$-.30-.10$

$-.21-.28$

$.01 \quad .09$

$.01 \quad .06$

$.02 \quad .06$
.03

$.03 \quad .00$

$.03-.04$

$.03-.08$

$.07-.03$

$-.01-.03$

$-.06-.04$

$-.18-.03$

$.00 \quad .04$

$-.03-.16$

$.14-.01$

$.23 \quad .02$

$.16-.08$

$.10 \quad .03$

$.05 \quad .05$

$.10 \quad .06$

$-.01 \quad .03$

$.09 \quad .04$

$\begin{array}{rr}.15 & .07\end{array}$

$-.03 \quad .02$

$-.05 \quad .03$

$.01 \quad .02$

$.12 \quad .05$

$-.08 \quad .01$

$-.06 \quad .00$

thermodynamics:

Sum of electronic and zero-point Energies=

$-3156.319171$

Sum of electronic and thermal Enthalpies=

$-3156.296876$

Sum of electronic and thermal Free Energies=

$-3156.374855$

Total free energy in solution:

with all non electrostatic terms

$(\mathrm{a} \cdot \mathrm{u})=$. 


\section{With paratolualdehyde}

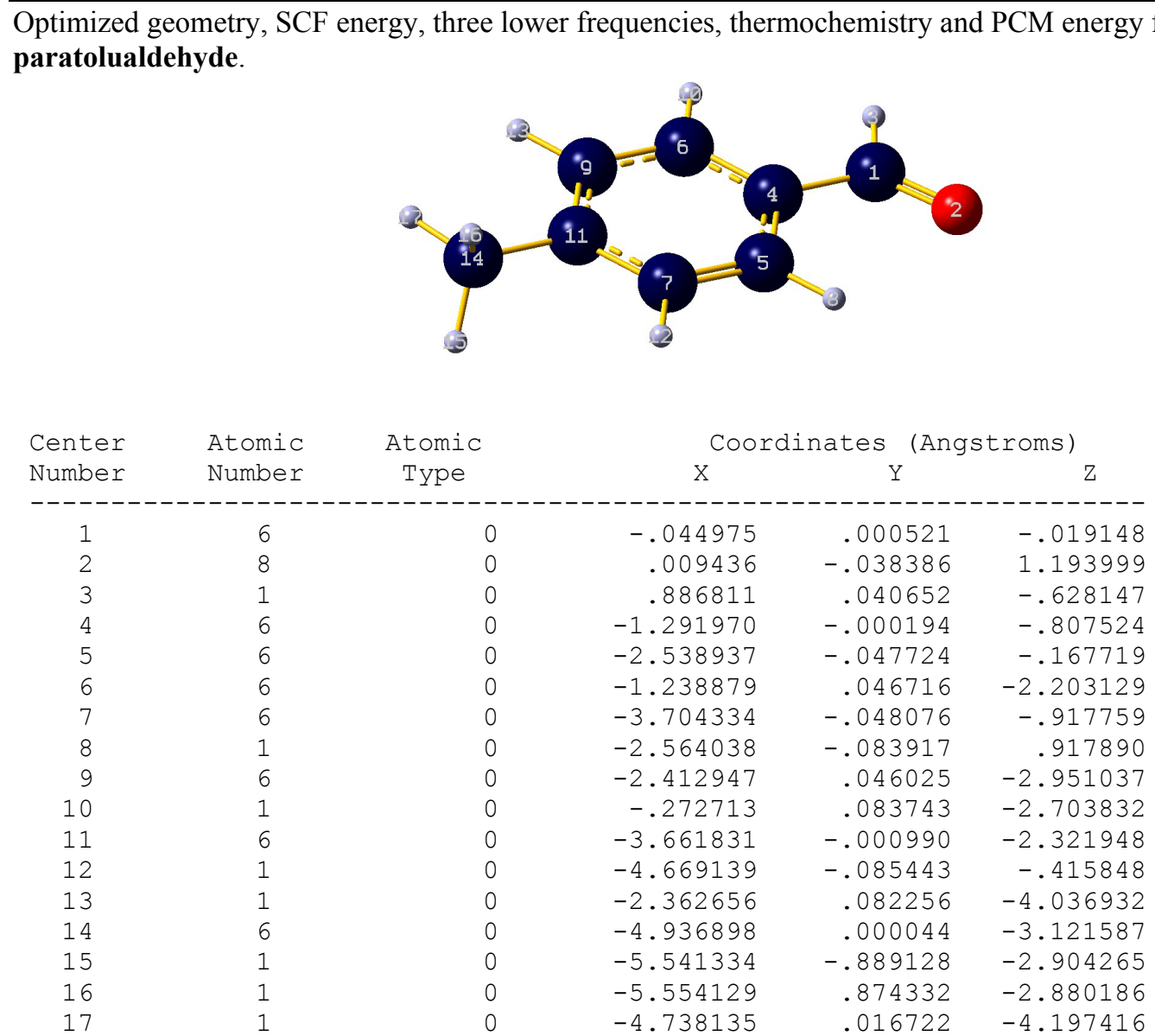

$\mathrm{HF}=-384.737556$

\begin{tabular}{|c|c|c|c|c|c|c|c|c|c|c|}
\hline & \multicolumn{3}{|c|}{1} & \multicolumn{3}{|c|}{2} & \multicolumn{3}{|c|}{3} \\
\hline & & \multicolumn{3}{|c|}{ A } & \multicolumn{3}{|c|}{ A } & \multicolumn{3}{|c|}{ A } \\
\hline \multicolumn{2}{|c|}{ Frequencies } & -- & \multicolumn{2}{|c|}{29.4567} & \multicolumn{3}{|c|}{99.3857} & \multicolumn{3}{|c|}{187.2847} \\
\hline \multicolumn{2}{|c|}{ Red. masses } & -- & \multicolumn{2}{|c|}{1.0508} & \multicolumn{3}{|c|}{5.1094} & \multicolumn{3}{|c|}{2.7152} \\
\hline \multicolumn{2}{|c|}{ Frc consts } & -- & \multirow{2}{*}{\multicolumn{2}{|c|}{$\begin{array}{l}.0005 \\
.4246\end{array}$}} & \multicolumn{3}{|c|}{.0297} & \multicolumn{3}{|c|}{.0561} \\
\hline \multicolumn{2}{|c|}{ IR Inten } & -- & & & \multicolumn{3}{|c|}{1.4777} & \multicolumn{3}{|c|}{8.8584} \\
\hline Atom & AN & X & $\mathrm{Y}$ & Z & $\mathrm{x}$ & $\mathrm{Y}$ & Z & $\mathrm{x}$ & $Y$ & Z \\
\hline 1 & 6 & .00 & -.02 & .00 & .00 & .00 & .00 & -.01 & .25 & .01 \\
\hline 2 & 8 & .00 & .00 & .00 & -.01 & .33 & .01 & .00 & -.14 & .00 \\
\hline 3 & 1 & .00 & -.04 & .00 & .00 & -.13 & .00 & -.01 & .76 & .03 \\
\hline 4 & 6 & .00 & -.01 & .00 & .00 & -.19 & -.01 & .00 & .07 & .00 \\
\hline 5 & 6 & .00 & .03 & .00 & .01 & -.25 & -.01 & .00 & -.01 & .00 \\
\hline 6 & 6 & .00 & -.02 & .00 & .00 & -.13 & .00 & .00 & -.06 & .00 \\
\hline 7 & 6 & .00 & .04 & .00 & .00 & -.17 & -.01 & .00 & -.05 & .00 \\
\hline 8 & 1 & .00 & .05 & .00 & .01 & -.30 & -.01 & .00 & .04 & .00 \\
\hline 9 & 6 & .00 & -.01 & .00 & .00 & -.02 & .00 & .00 & -.18 & -.01 \\
\hline 10 & 1 & .00 & -.04 & .00 & .00 & -.11 & .00 & .00 & -.04 & -.01 \\
\hline 11 & 6 & .00 & .02 & .00 & .00 & .01 & .00 & .00 & -.07 & .00 \\
\hline 12 & 1 & .00 & .07 & .00 & .00 & -.20 & -.01 & .00 & -.01 & .00 \\
\hline 13 & 1 & .00 & -.01 & .00 & .00 & .09 & .00 & .00 & -.25 & -.01 \\
\hline 14 & 6 & .00 & -.02 & .00 & -.01 & .27 & .01 & .00 & .14 & .01 \\
\hline 15 & 1 & .29 & -.33 & -.41 & -.07 & .27 & -.19 & -.08 & .16 & -.14 \\
\hline 16 & 1 & -.29 & -.34 & .40 & .06 & .27 & .22 & .08 & .16 & .16 \\
\hline 17 & 1 & .00 & .52 & .01 & -.01 & .51 & .01 & -.01 & .30 & .01 \\
\hline
\end{tabular}

thermodynamics:

Sum of electronic and zero-point Energies= 
Total free energy in solution:

with all non electrostatic terms

$($ a.u. $)=-384.737169$

Optimized geometry, SCF energy, three lower frequencies, thermochemistry and PCM energy for $\mathbf{T S}_{\gamma}$.



\begin{tabular}{|c|c|c|c|c|c|}
\hline Center & Atomic & Atomic & $\mathrm{COO}$ & inates (An & Eroms) \\
\hline Number & Number & Type & $\mathrm{x}$ & $\mathrm{Y}$ & Z \\
\hline 1 & 6 & 0 & -.637002 & 1.997629 & -.888980 \\
\hline 2 & 1 & 0 & -1.058266 & 1.434876 & -1.718022 \\
\hline 3 & 1 & 0 & -1.299245 & 2.740060 & -.451433 \\
\hline 4 & 6 & 0 & .733483 & 2.210150 & -.862249 \\
\hline 5 & 1 & 0 & 1.115856 & 2.984165 & -.192861 \\
\hline 6 & 6 & 0 & 1.687169 & 1.364014 & -1.475601 \\
\hline 7 & 1 & 0 & 2.679891 & 1.787392 & -1.623579 \\
\hline 8 & 1 & 0 & 1.337291 & .775783 & -2.325899 \\
\hline 9 & 50 & 0 & 2.313697 & -.296672 & .099534 \\
\hline 10 & 6 & 0 & 2.162970 & .232276 & 2.186850 \\
\hline 11 & 1 & 0 & 1.307337 & -.256774 & 2.660838 \\
\hline 12 & 1 & 0 & 3.078759 & -.077748 & 2.701610 \\
\hline 13 & 1 & 0 & 2.062458 & 1.315451 & 2.317597 \\
\hline 14 & 6 & 0 & 2.197887 & -2.366522 & -.475896 \\
\hline 15 & 1 & 0 & 2.135326 & -2.455381 & -1.566016 \\
\hline 16 & 1 & 0 & 3.098386 & -2.895587 & -.146235 \\
\hline 17 & 1 & 0 & 1.313990 & -2.837452 & -.040119 \\
\hline 18 & 6 & 0 & 4.419644 & .168956 & -.317832 \\
\hline 19 & 1 & 0 & 4.662160 & 1.205009 & -.052920 \\
\hline 20 & 1 & 0 & 5.069182 & -.491111 & .270352 \\
\hline 21 & 1 & 0 & 4.658210 & .020259 & -1.377501 \\
\hline 22 & 1 & 0 & -.551225 & 1.030118 & 1.332215 \\
\hline 23 & 6 & 0 & -.786001 & .426916 & .439774 \\
\hline 24 & 8 & 0 & .042724 & -.472307 & .075735 \\
\hline 25 & 6 & 0 & -2.246107 & .173034 & .256390 \\
\hline 26 & 6 & 0 & -2.689453 & -.848305 & -.590751 \\
\hline 27 & 6 & 0 & -3.192685 & .922101 & .961802 \\
\hline 28 & 6 & 0 & -4.048620 & -1.101467 & -.733281 \\
\hline 29 & 1 & 0 & -1.951457 & -1.442390 & -1.121878 \\
\hline 30 & 6 & 0 & -4.552122 & .661231 & .817584 \\
\hline 31 & 1 & 0 & -2.862665 & 1.714349 & 1.632160 \\
\hline 32 & 6 & 0 & -5.005391 & -.354535 & -.032170 \\
\hline 33 & 1 & 0 & -4.377499 & -1.899714 & -1.396165 \\
\hline 34 & 1 & 0 & -5.274369 & 1.253095 & 1.376406 \\
\hline 35 & 6 & 0 & -6.474007 & -.660596 & -.164514 \\
\hline 36 & 1 & 0 & -6.769589 & -1.478133 & .506738 \\
\hline 37 & 1 & 0 & -7.090678 & .208064 & .088914 \\
\hline 38 & 1 & 0 & -6.729105 & -.971581 & -1.183567 \\
\hline
\end{tabular}

$\mathrm{HF}=-625.0644822$ 


\begin{tabular}{|c|c|c|c|c|c|c|c|c|c|c|}
\hline \multicolumn{2}{|c|}{ Frequencies } & \multicolumn{3}{|c|}{-345.2692} & \multicolumn{3}{|c|}{25.7355} & \multicolumn{3}{|c|}{29.5703} \\
\hline Red. & masses & -- & 10.346 & & & 1.86 & & & 1.265 & \\
\hline Frc & consts & -- & .726 & & & .00 & & & .000 & \\
\hline IR Ir & nten & -- & 370.955 & & & .15 & & & .275 & \\
\hline Atom & AN & $\mathrm{X}$ & Y & Z & $\mathrm{X}$ & Y & Z & $\mathrm{X}$ & Y & Z \\
\hline 1 & 6 & .02 & .48 & -.38 & .01 & -.02 & -.04 & .00 & -.02 & -.03 \\
\hline 2 & 1 & -.05 & .06 & -.03 & .01 & -.03 & -.04 & .00 & -.02 & -.03 \\
\hline 3 & 1 & -.03 & .30 & -.17 & .00 & -.03 & -.05 & .01 & -.01 & -.04 \\
\hline 4 & 6 & -.07 & .04 & .01 & .01 & -.02 & -.03 & .00 & -.02 & -.04 \\
\hline 5 & 1 & -.04 & -.04 & .08 & .00 & -.01 & -.03 & .01 & -.01 & -.05 \\
\hline 6 & 6 & .01 & -.22 & .18 & .01 & -.02 & -.02 & .00 & -.03 & -.03 \\
\hline 7 & 1 & -.08 & -.03 & -.01 & .01 & -.01 & -.01 & .00 & -.03 & -.04 \\
\hline 8 & 1 & -.03 & .05 & .00 & .02 & -.02 & -.02 & .00 & -.04 & -.02 \\
\hline 9 & 50 & .05 & .03 & -.03 & .00 & .00 & .00 & .00 & .00 & .00 \\
\hline 10 & 6 & -.04 & -.01 & -.02 & -.09 & -.06 & .01 & -.05 & -.01 & .00 \\
\hline 11 & 1 & -.02 & -.01 & .03 & -.12 & -.07 & -.04 & -.06 & -.01 & -.02 \\
\hline 12 & 1 & -.03 & -.01 & -.04 & -.12 & -.09 & .04 & -.06 & -.02 & .02 \\
\hline 13 & 1 & -.03 & -.01 & -.01 & -.08 & -.06 & .04 & -.04 & -.01 & .01 \\
\hline 14 & 6 & -.03 & .01 & .01 & .09 & .02 & -.06 & .05 & .00 & -.01 \\
\hline 15 & 1 & -.03 & .01 & .01 & .31 & .04 & -.08 & .20 & .00 & -.02 \\
\hline 16 & 1 & -.02 & .02 & .01 & .03 & .01 & .10 & .01 & .00 & .11 \\
\hline 17 & 1 & -.01 & -.02 & .01 & .01 & .01 & -.25 & -.01 & .01 & -.12 \\
\hline 18 & 6 & .07 & -.06 & .07 & .00 & .08 & .12 & .00 & .04 & .06 \\
\hline 19 & 1 & .03 & -.04 & .03 & -.05 & .09 & .12 & -.03 & .05 & .05 \\
\hline 20 & 1 & .18 & .00 & .01 & .00 & .11 & .16 & .00 & .07 & .09 \\
\hline 21 & 1 & .02 & -.03 & .05 & .07 & .08 & .13 & .04 & .03 & .07 \\
\hline 22 & 1 & .06 & -.01 & .00 & .03 & -.04 & -.05 & .02 & -.01 & -.03 \\
\hline 23 & 6 & -.11 & -.39 & .32 & .01 & -.03 & -.05 & .01 & -.01 & -.03 \\
\hline 24 & 8 & -.15 & -.09 & .07 & .00 & -.03 & -.07 & .00 & -.02 & -.03 \\
\hline 25 & 6 & -.04 & -.03 & .03 & .00 & -.01 & -.03 & .00 & .00 & -.01 \\
\hline 26 & 6 & -.03 & -.02 & .00 & -.03 & -.01 & -.01 & -.02 & .01 & -.02 \\
\hline 27 & 6 & -.02 & .00 & .02 & .03 & .00 & -.01 & .02 & -.01 & .01 \\
\hline 28 & 6 & -.02 & .00 & -.02 & -.03 & .01 & .02 & -.02 & .02 & .00 \\
\hline 29 & 1 & -.02 & -.02 & .01 & -.05 & -.02 & -.03 & -.03 & .01 & -.03 \\
\hline 30 & 6 & -.02 & .01 & .00 & .02 & .02 & .02 & .01 & .00 & .03 \\
\hline 31 & 1 & -.01 & -.01 & .02 & .05 & .00 & -.02 & .03 & -.02 & .02 \\
\hline 32 & 6 & -.01 & .01 & .00 & -.01 & .02 & .03 & .00 & .01 & .03 \\
\hline 33 & 1 & -.03 & .01 & -.02 & -.06 & .01 & .03 & -.03 & .02 & .00 \\
\hline 34 & 1 & -.02 & .02 & -.02 & .04 & .03 & .03 & .02 & -.01 & .05 \\
\hline 35 & 6 & -.01 & .01 & .00 & -.02 & .04 & .08 & -.01 & .02 & .03 \\
\hline 36 & 1 & .01 & .00 & .00 & -.09 & .33 & .41 & .10 & -.34 & -.36 \\
\hline 37 & 1 & -.01 & .00 & .01 & .01 & .17 & -.29 & -.01 & -.13 & .53 \\
\hline 38 & 1 & .00 & .00 & .00 & .02 & -.36 & .20 & -.10 & .53 & -.11 \\
\hline
\end{tabular}

thermodynamics:

Sum of electronic and zero-point Energies=

$-624.745205$

Sum of electronic and thermal Enthalpies=

$-624.723282$

Sum of electronic and thermal Free Energies=

$-624.797170$

Total free energy in solution:

with all non electrostatic terms

$(\mathrm{a} \cdot \mathrm{u} \cdot)=$

$-625.046206$

Optimized geometry, SCF energy, three lower frequencies, thermochemistry and PCM energy for product. 


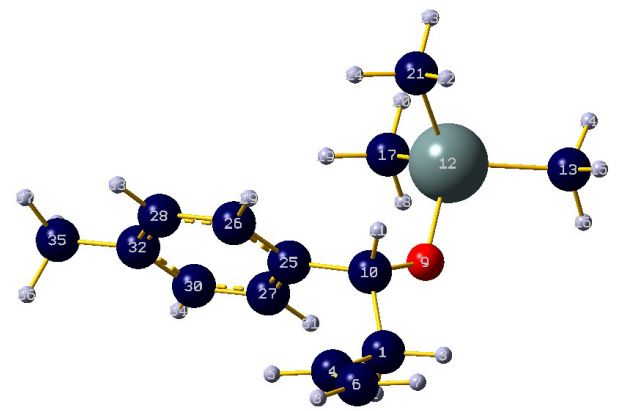



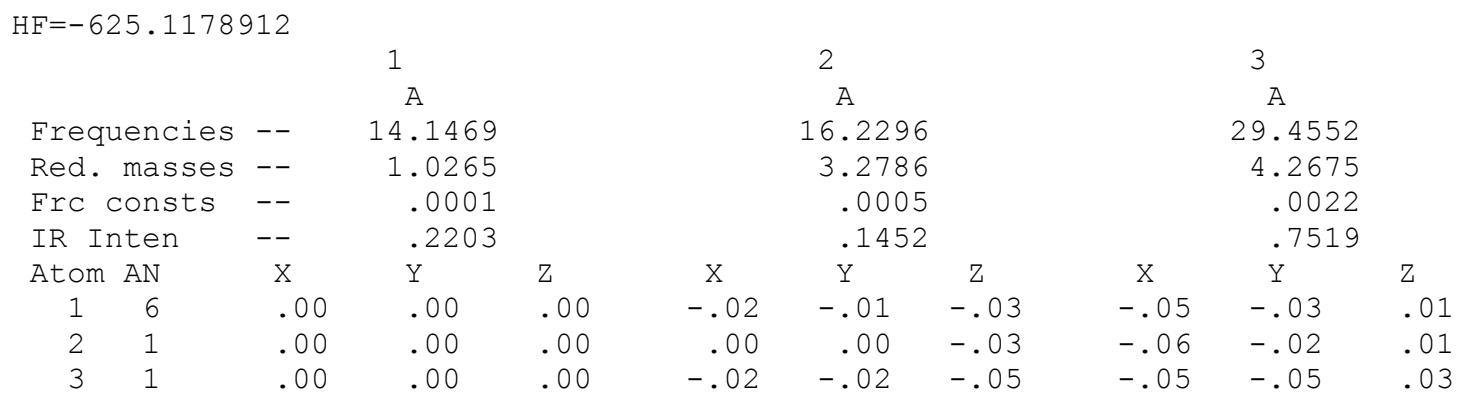

S17 


\begin{tabular}{|c|c|c|c|c|c|c|c|c|c|c|}
\hline 4 & 6 & .00 & .00 & .01 & -.02 & -.02 & -.05 & -.03 & -.04 & -.01 \\
\hline 5 & 1 & .00 & .00 & .01 & -.01 & -.01 & -.03 & -.02 & -.01 & -.01 \\
\hline 6 & 6 & .00 & .01 & .01 & -.02 & -.04 & -.07 & -.02 & -.08 & -.04 \\
\hline 7 & 1 & .00 & .01 & .01 & -.03 & -.06 & -.09 & -.03 & -.12 & -.05 \\
\hline 8 & 1 & -.01 & .01 & .01 & -.02 & -.05 & -.08 & .00 & -.09 & -.06 \\
\hline 9 & 8 & .00 & .00 & .00 & -.03 & -.01 & .01 & -.08 & -.03 & .06 \\
\hline 10 & 6 & .00 & .00 & .00 & -.03 & -.01 & .00 & -.06 & -.04 & .03 \\
\hline 11 & 1 & .00 & .00 & .00 & -.05 & -.03 & .00 & -.05 & -.06 & .03 \\
\hline 12 & 50 & .00 & .00 & .00 & .02 & -.01 & -.01 & .05 & -.03 & .01 \\
\hline 13 & 6 & .00 & .00 & .01 & -.02 & .02 & .21 & .12 & .09 & -.09 \\
\hline 14 & 1 & -.01 & .00 & .01 & -.02 & .01 & .33 & .19 & .09 & -.09 \\
\hline 15 & 1 & .00 & .00 & .01 & .04 & -.07 & .22 & .06 & .14 & -.13 \\
\hline 16 & 1 & -.01 & .00 & .01 & -.09 & .14 & .20 & .15 & .11 & -.11 \\
\hline 17 & 6 & -.01 & .00 & -.01 & -.11 & -.03 & -.18 & .14 & -.14 & .04 \\
\hline 18 & 1 & -.01 & .00 & -.01 & -.21 & -.08 & -.15 & .20 & -.20 & .03 \\
\hline 19 & 1 & -.01 & .00 & -.02 & -.09 & -.02 & -.25 & .13 & -.15 & .12 \\
\hline 20 & 1 & -.01 & .00 & -.01 & -.11 & -.03 & -.24 & .15 & -.14 & -.01 \\
\hline 21 & 6 & .01 & .00 & -.01 & .23 & .00 & -.07 & -.01 & -.01 & .02 \\
\hline 22 & 1 & .01 & .01 & .00 & .28 & .03 & .01 & -.04 & .02 & .00 \\
\hline 23 & 1 & .01 & .00 & -.01 & .29 & .00 & -.10 & .00 & -.01 & .01 \\
\hline 24 & 1 & .01 & .00 & -.01 & .23 & -.03 & -.16 & -.03 & -.03 & .06 \\
\hline 25 & 6 & .00 & .00 & .00 & -.03 & .00 & .02 & -.07 & .00 & .01 \\
\hline 26 & 6 & .00 & -.01 & .00 & -.04 & .00 & .03 & -.04 & .12 & .02 \\
\hline 27 & 6 & .00 & .00 & .00 & -.01 & .02 & .03 & -.09 & -.07 & -.01 \\
\hline 28 & 6 & .00 & -.02 & .00 & -.04 & .03 & .05 & -.04 & .18 & .00 \\
\hline 29 & 1 & .00 & -.02 & .00 & -.06 & -.01 & .02 & -.02 & .17 & .03 \\
\hline 30 & 6 & .00 & .00 & .00 & -.01 & .04 & .05 & -.10 & -.01 & -.03 \\
\hline 31 & 1 & .00 & .01 & .00 & .00 & .02 & .02 & -.12 & -.16 & -.02 \\
\hline 32 & 6 & .00 & -.01 & .00 & -.02 & .05 & .06 & -.07 & .12 & -.02 \\
\hline 33 & 1 & .00 & -.03 & .00 & -.05 & .03 & .06 & -.01 & .28 & .01 \\
\hline 34 & 1 & .00 & .00 & .00 & .01 & .06 & .06 & -.12 & -.06 & -.05 \\
\hline 35 & 6 & .00 & .02 & .00 & -.01 & .07 & .09 & -.06 & .21 & -.03 \\
\hline 36 & 1 & -.05 & .30 & -.51 & .01 & .04 & .14 & -.02 & .26 & -.10 \\
\hline 37 & 1 & -.03 & -.53 & .03 & -.03 & .13 & .09 & -.06 & .18 & -.03 \\
\hline 38 & 1 & .08 & .35 & .48 & -.03 & .04 & .05 & -.11 & .27 & .02 \\
\hline
\end{tabular}

thermodynamics:

Sum of electronic and zero-point Energies=

$-624.796362$

Sum of electronic and thermal Enthalpies=

$-624.773651$

Sum of electronic and thermal Free Energies=

$-624.852171$

Total free energy in solution:

with all non electrostatic terms

$(\mathrm{a} \cdot \mathrm{u} \cdot)=$

$-625.095919$

\section{With paranitrobenzaldehyde}

Optimized geometry, SCF energy, three lower frequencies, thermochemistry and PCM energy for paranitrobenzaldehyde.



\begin{tabular}{lcccr} 
Center & Atomic & Atomic & \multicolumn{2}{c}{ Coordinates (Angstroms) } \\
Number & Number & Type & X & Y \\
$------n$ &
\end{tabular}




$\begin{array}{rrrrrr}1 & 6 & 0 & -.050750 & .000047 & -.025091 \\ 2 & 8 & 0 & -.012350 & .015938 & 1.186336 \\ 3 & 1 & 0 & .883040 & -.015877 & -.628510 \\ 4 & 6 & 0 & -1.305332 & -.000035 & -.817907 \\ 5 & 6 & 0 & -2.545034 & .019101 & -.164885 \\ 6 & 6 & 0 & -1.245439 & -.019333 & -2.215490 \\ 7 & 6 & 0 & -3.719976 & .019155 & -.902493 \\ 8 & 1 & 0 & -2.562440 & .033714 & .920748 \\ 9 & 6 & 0 & -2.414519 & -.019601 & -2.968192 \\ 10 & 1 & 0 & -.279300 & -.034245 & -2.715252 \\ 11 & 6 & 0 & -3.631279 & -.000275 & -2.293846 \\ 12 & 1 & 0 & -4.695450 & .033825 & -.030447 \\ 13 & 1 & 0 & -2.403142 & -.034232 & -4.051653 \\ 14 & 7 & 0 & -4.873985 & -.000208 & -3.082055 \\ 15 & 8 & 0 & -5.931279 & .017325 & -2.466510 \\ 16 & 8 & 0 & -4.767978 & -.017623 & -4.301172\end{array}$

$\mathrm{HF}=-549.851239$



thermodynamics:

Sum of electronic and zero-point Energies=

$-549.738064$

Sum of electronic and thermal Enthalpies=

$-549.728309$

Sum of electronic and thermal Free Energies=

$-549.772941$

Total free energy in solution:

with all non electrostatic terms

$($ a.u. $)=\quad-549.855562$

Optimized geometry, SCF energy, three lower frequencies, thermochemistry and PCM energy for $\mathbf{T} \mathbf{S}_{\gamma}$.

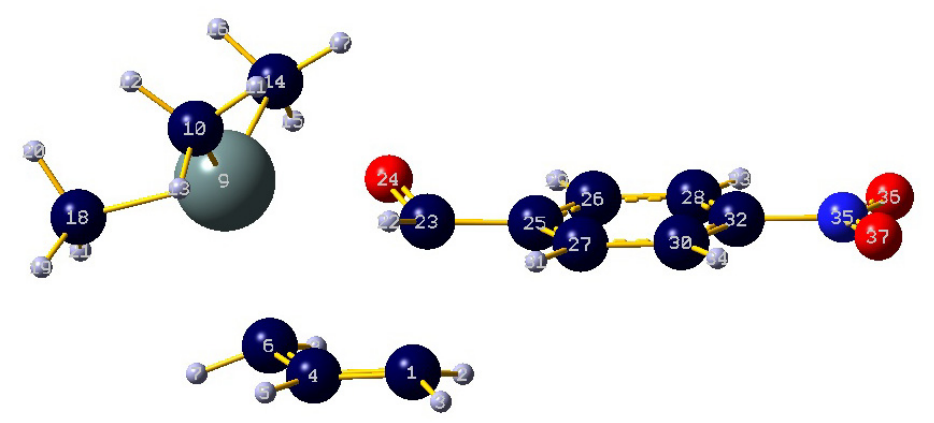




\begin{tabular}{|c|c|c|c|c|c|}
\hline Center & Atomic & Atomic & Coor & inates (An & Eroms ) \\
\hline Number & Number & Type & $\mathrm{X}$ & Y & Z \\
\hline 1 & 6 & 0 & .084082 & -1.907629 & -1.15343 \\
\hline 2 & 1 & 0 & .536795 & -1.232305 & -1.874769 \\
\hline 3 & 1 & 0 & .713433 & -2.730746 & -.825558 \\
\hline 4 & 6 & 0 & -1.292156 & -2.061806 & -1.162198 \\
\hline 5 & 1 & 0 & -1.712322 & -2.915380 & -.625908 \\
\hline 6 & 6 & 0 & -2.206273 & -1.096776 & -1.651877 \\
\hline 7 & 1 & 0 & -3.202544 & -1.467234 & -1.891964 \\
\hline 8 & 1 & 0 & -1.818036 & -.396561 & -2.393745 \\
\hline 9 & 50 & 0 & -2.873014 & .307468 & .12909 \\
\hline 10 & 6 & 0 & -2.789615 & -.554518 & 2.10314 \\
\hline 11 & 1 & 0 & -1.905629 & -.221647 & 2.65369 \\
\hline 12 & 1 & 0 & -3.684731 & -.253313 & 2.65800 \\
\hline 13 & 1 & 0 & -2.785362 & -1.649135 & 2.05765 \\
\hline 14 & 6 & 0 & -2.651954 & 2.430311 & -.11663 \\
\hline 15 & 1 & 0 & -2.623007 & 2.686847 & -1.181127 \\
\hline 16 & 1 & 0 & -3.509966 & 2.944618 & .32922 \\
\hline 17 & 1 & 0 & -1.730189 & 2.779983 & .35263 \\
\hline 18 & 6 & 0 & -4.970752 & -.015613 & -.41903 \\
\hline 19 & 1 & 0 & -5.252445 & -1.071234 & -.32719 \\
\hline 20 & 1 & 0 & -5.620232 & .565779 & .24681 \\
\hline 21 & 1 & 0 & -5.170863 & .304902 & -1.44805 \\
\hline 22 & 1 & 0 & -.012973 & -1.285659 & 1.21242 \\
\hline 23 & 6 & 0 & .244545 & -.543739 & .43904 \\
\hline 24 & 8 & 0 & -.549395 & .418859 & .21472 \\
\hline 25 & 6 & 0 & 1.713811 & -.317613 & .28094 \\
\hline 26 & 6 & 0 & 2.177698 & .814178 & -.40224 \\
\hline 27 & 6 & 0 & 2.634147 & -1.199866 & .86170 \\
\hline 28 & 6 & 0 & 3.539478 & 1.053594 & -.52293 \\
\hline 29 & 1 & 0 & 1.451491 & 1.503770 & -.82073 \\
\hline 30 & 6 & 0 & 3.999522 & -.970935 & .75333 \\
\hline 31 & 1 & 0 & 2.276628 & -2.070275 & 1.40768 \\
\hline 32 & 6 & 0 & 4.432743 & .154267 & .05618 \\
\hline 33 & 1 & 0 & 3.922802 & 1.921880 & -1.04648 \\
\hline 34 & 1 & 0 & 4.729865 & -1.637899 & 1.196414 \\
\hline 35 & 7 & 0 & 5.871101 & .402850 & -.064654 \\
\hline 36 & 8 & 0 & 6.224617 & 1.398883 & -.684571 \\
\hline 37 & 8 & 0 & 6.632106 & -.401853 & .460197 \\
\hline
\end{tabular}

$\mathrm{HF}=-790.1825347$

\begin{tabular}{|c|c|c|c|c|c|c|c|c|c|}
\hline & & 1 & & & 2 & & & 3 & \\
\hline & & A & & & A & & & A & \\
\hline Frequencies & -- & -308.4673 & & & 35.24 & & & 37.211 & \\
\hline Red. masses & -- & 10.0738 & & & 3.92 & & & 4.7722 & \\
\hline Frc consts & -- & .5648 & & & .00 & & & .003 & \\
\hline IR Inten & -- & 286.5928 & & & .47 & & & .470 & \\
\hline Atom AN & $\mathrm{x}$ & Y & Z & $\mathrm{x}$ & Y & Z & $\mathrm{x}$ & Y & Z \\
\hline 6 & .02 & .41 & .45 & -.03 & .06 & -.13 & .04 & .06 & .04 \\
\hline 1 & -.06 & .08 & .07 & -.07 & .08 & -.13 & .09 & .05 & .06 \\
\hline 1 & -.03 & .29 & .25 & .01 & .06 & -.16 & .01 & .05 & .07 \\
\hline 6 & -.07 & .04 & .00 & -.02 & .03 & -.07 & .03 & .09 & -.04 \\
\hline 1 & -.04 & -.02 & -.08 & .02 & .01 & -.06 & -.01 & .08 & -.08 \\
\hline 6 & .00 & -.18 & -.21 & -.07 & .01 & -.02 & .08 & .11 & -.08 \\
\hline 1 & -.09 & -.04 & .00 & -.07 & -.01 & .03 & .09 & .14 & -.16 \\
\hline 1 & -.03 & .02 & -.02 & -.12 & .02 & -.03 & .15 & .14 & -.01 \\
\hline 950 & .05 & .02 & .04 & .00 & .00 & .02 & .00 & -.01 & .00 \\
\hline 10 & -.04 & -.01 & .01 & .15 & .07 & .04 & .01 & -.13 & -.05 \\
\hline 11 & -.02 & -.01 & -.03 & .19 & .09 & -.03 & -.02 & -.12 & -.01 \\
\hline 12 & -.03 & -.02 & .02 & .19 & .09 & .09 & -.02 & -.20 & -.05 \\
\hline 13 & -.03 & -.01 & .00 & .15 & .07 & .08 & .06 & -.13 & -.11 \\
\hline 14 & -.02 & .01 & -.01 & -.10 & -.01 & -.09 & -.11 & .02 & .17 \\
\hline 15 & -.03 & .01 & -.01 & -.34 & -.03 & -.10 & -.16 & .12 & .19 \\
\hline 16 & -.02 & .01 & -.01 & -.02 & -.02 & .09 & -.12 & -.07 & .25 \\
\hline 17 & -.01 & -.02 & -.02 & -.01 & .03 & -.29 & -.12 & .02 & .17 \\
\hline 18 & .07 & -.05 & -.07 & -.03 & -.12 & .20 & .02 & -.09 & -.0 \\
\hline
\end{tabular}




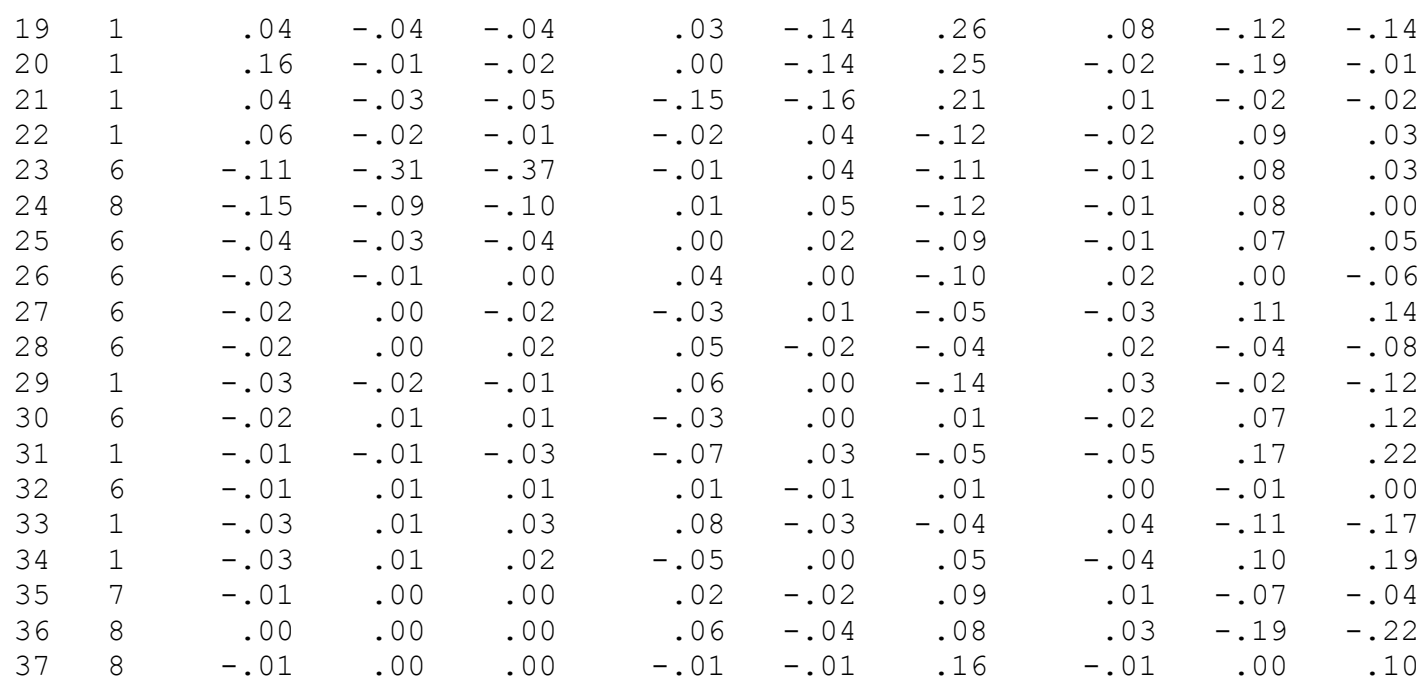

thermodynamics:

Sum of electronic and zero-point Energies=

$-789.887919$

Sum of electronic and thermal Enthalpies=

$-789.865388$

Sum of electronic and thermal Free Energies=

$-789.940372$

Total free energy in solution:

with all non electrostatic terms

$(\mathrm{a} \cdot \mathrm{u})=$.

Optimized geometry, SCF energy, three lower frequencies, thermochemistry and PCM energy for product.

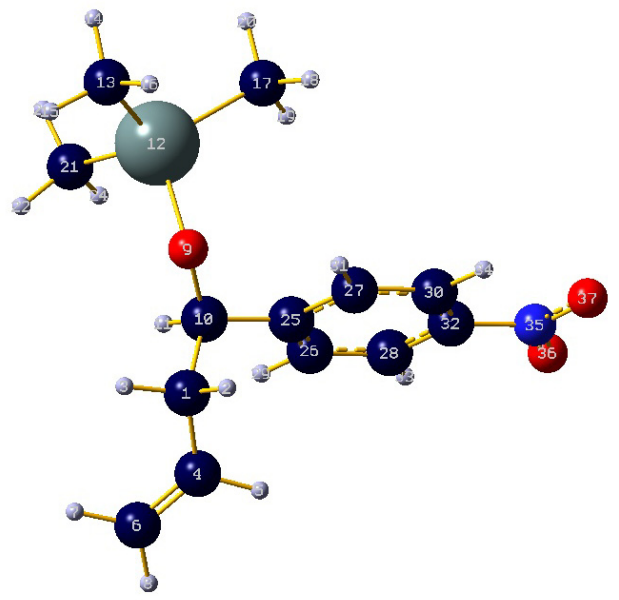

\begin{tabular}{|c|c|c|c|c|c|}
\hline \multirow{2}{*}{$\begin{array}{l}\text { Center } \\
\text { Number }\end{array}$} & \multirow{2}{*}{$\begin{array}{l}\text { Atomic } \\
\text { Number }\end{array}$} & \multirow{2}{*}{$\begin{array}{l}\text { Atomic } \\
\text { Type }\end{array}$} & \multicolumn{3}{|c|}{ Coordinates (Angstroms) } \\
\hline & & & $\mathrm{x}$ & $\mathrm{Y}$ & Z \\
\hline 1 & 6 & 0 & -.125750 & -.091181 & -.053821 \\
\hline 2 & 1 & 0 & -.166542 & .069468 & 1.032799 \\
\hline 3 & 1 & 0 & .922711 & -.279182 & -.312863 \\
\hline 4 & 6 & 0 & -.636101 & 1.115301 & -.783081 \\
\hline 5 & 1 & 0 & -1.625681 & 1.473615 & -.497168 \\
\hline 6 & 6 & 0 & .029806 & 1.759210 & -1.743052 \\
\hline 7 & 1 & 0 & 1.022758 & 1.444385 & -2.060338 \\
\hline 8 & 1 & 0 & -.386273 & 2.631018 & -2.241837 \\
\hline 9 & 8 & 0 & -.258742 & -2.426217 & .361791 \\
\hline 10 & 6 & 0 & -.899242 & -1.397867 & -.349961 \\
\hline 11 & 1 & 0 & -.847134 & -1.564675 & -1.440150 \\
\hline 12 & 50 & 0 & -.312146 & -4.329386 & -.159023 \\
\hline 13 & 6 & 0 & 1.325271 & -5.135314 & .956781 \\
\hline
\end{tabular}




14
15
16
17
18
19
20
21
22
23
24
25
26
27
28
29
30
31
32
33
34
35
36
37



$-6.227332$

$-4.739186$

$-4.862153$

$-5.189673$

$-5.009364$

$-4.760379$

$-6.271816$

$-4.419888$

$-3.834369$

$-5.458393$

$-4.038684$

$-1.277968$

$-.955229$

$-1.468659$

$-.817801$

$-.807886$

$-1.335847$

$-1.733656$

$-1.010988$

$-.571504$

$-1.479013$

$-.874524$

$-.587505$

$-1.055345$
.882619

.582822

2.012012

.402558

1. 461113

$-.189584$

.232683

$-2.286604$

$-2.588173$

$-2.596388$

$-2.817204$

.031044

$-.924620$

1. 361809

$-.569154$

$-1.960869$

1.736296

2.099248

.761414

$-1.297782$

2.761054

1.146255

.267619

2. 324000

$\mathrm{HF}=-790.2374466$

\begin{tabular}{|c|c|c|c|c|c|c|c|c|c|c|}
\hline & \multicolumn{3}{|c|}{1} & \multicolumn{3}{|c|}{2} & \multicolumn{3}{|c|}{3} \\
\hline & & \multicolumn{3}{|c|}{ A } & \multicolumn{3}{|c|}{ A } & \multicolumn{3}{|c|}{ A } \\
\hline Freq & dencies & -- & 15.61 & & & 21.80 & & & 33.82 & \\
\hline Red. & masses & -- & 3.33 & & & 5.69 & & & 6.19 & \\
\hline Frc & consts & -- & .00 & & & .00 & & & .00 & \\
\hline IR I & iten & -- & .12 & & & .88 & & & .14 & \\
\hline Atom & AN & $\mathrm{X}$ & Y & Z & $\mathrm{x}$ & Y & Z & $\mathrm{x}$ & Y & Z \\
\hline 1 & 6 & -.02 & -.01 & -.04 & -.06 & -.05 & .01 & .03 & .03 & -.05 \\
\hline 2 & 1 & -.01 & .00 & -.04 & -.06 & -.04 & .01 & .03 & .04 & -.06 \\
\hline 3 & 1 & -.03 & -.02 & -.05 & -.06 & -.06 & .01 & .03 & .03 & -.06 \\
\hline 4 & 6 & -.03 & -.02 & -.05 & -.05 & -.05 & -.01 & .03 & .03 & -.06 \\
\hline 5 & 1 & -.02 & -.01 & -.04 & -.04 & -.02 & .01 & .05 & .06 & -.04 \\
\hline 6 & 6 & -.04 & -.04 & -.07 & -.06 & -.09 & -.04 & .02 & -.01 & -.11 \\
\hline 7 & 1 & -.05 & -.05 & -.08 & -.07 & -.12 & -.05 & .00 & -.04 & -.13 \\
\hline 8 & 1 & -.05 & -.05 & -.08 & -.05 & -.09 & -.05 & .02 & -.01 & -.11 \\
\hline 9 & 8 & -.02 & -.01 & .00 & -.08 & -.04 & .04 & .04 & .04 & -.05 \\
\hline 10 & 6 & -.03 & -.02 & -.01 & -.07 & -.05 & .02 & .03 & .03 & -.04 \\
\hline 11 & 1 & -.04 & -.03 & -.01 & -.07 & -.06 & .03 & .02 & .03 & -.04 \\
\hline 12 & 50 & .02 & .00 & -.01 & .07 & -.03 & .00 & -.03 & .02 & .02 \\
\hline 13 & 6 & -.11 & -.02 & .17 & .16 & .08 & -.07 & -.07 & .01 & .07 \\
\hline 14 & 1 & -.11 & -.02 & .18 & .22 & .08 & -.05 & -.11 & .01 & .13 \\
\hline 15 & 1 & -.07 & -.02 & .28 & .12 & .12 & -.13 & -.05 & -.04 & .06 \\
\hline 16 & 1 & -.23 & -.01 & .16 & .20 & .09 & -.07 & -.07 & .07 & .06 \\
\hline 17 & 6 & -.05 & .00 & -.24 & .15 & -.18 & .05 & -.06 & .10 & .05 \\
\hline 18 & 1 & -.17 & -.03 & -.25 & .20 & -.27 & .07 & -.09 & .19 & .02 \\
\hline 19 & 1 & .02 & .04 & -.31 & .09 & -.19 & .12 & -.04 & .06 & -.01 \\
\hline 20 & 1 & -.05 & .01 & -.28 & .20 & -.17 & -.03 & -.07 & .08 & .14 \\
\hline 21 & 6 & .25 & .01 & .02 & .01 & .04 & -.01 & -.02 & -.08 & .03 \\
\hline 22 & 1 & .28 & .02 & .12 & -.04 & .11 & -.03 & .02 & -.15 & .01 \\
\hline 23 & 1 & .30 & .01 & .03 & .08 & .05 & -.04 & -.10 & -.10 & .07 \\
\hline 24 & 1 & .31 & .00 & -.07 & -.04 & -.02 & .02 & .00 & -.04 & .01 \\
\hline 25 & 6 & -.02 & -.01 & .01 & -.07 & -.03 & .01 & .03 & .03 & -.03 \\
\hline 26 & 6 & -.03 & .00 & .02 & -.04 & .10 & .03 & .04 & .18 & .01 \\
\hline 27 & 6 & -.01 & -.01 & .01 & -.10 & -.11 & -.01 & .02 & -.15 & -.05 \\
\hline 28 & 6 & -.03 & .02 & .03 & -.04 & .15 & .02 & .04 & .16 & .03 \\
\hline 29 & 1 & -.04 & .00 & .01 & -.02 & .16 & .04 & .05 & .32 & .04 \\
\hline 30 & 6 & -.01 & .01 & .02 & -.10 & -.06 & -.01 & .02 & -.19 & -.04 \\
\hline 31 & 1 & -.01 & -.01 & .00 & -.12 & -.20 & -.02 & .02 & -.25 & -.09 \\
\hline 32 & 6 & -.01 & .02 & .04 & -.07 & .07 & .00 & .03 & -.03 & .00 \\
\hline
\end{tabular}




\begin{tabular}{|c|c|c|c|c|c|c|c|c|c|}
\hline 33 & -.03 & .02 & .04 & -.01 & .25 & .03 & .05 & .28 & .06 \\
\hline 34 & .00 & .01 & .03 & -.12 & -.12 & -.03 & .01 & -.33 & -.07 \\
\hline 7 & -.01 & .03 & .05 & -.06 & .13 & .00 & .03 & -.08 & .01 \\
\hline 8 & -.01 & .04 & .06 & -.03 & .27 & .01 & .05 & .17 & 06 \\
\hline 8 & & & & - 09 & .04 & -.02 & .00 & 38 & \\
\hline
\end{tabular}

thermodynamics:

Sum of electronic and zero-point Energies=

$-789.940671$

Sum of electronic and thermal Enthalpies=

$-789.917319$

Sum of electronic and thermal Free Energies=

$-789.997154$

Total free energy in solution:

with all non electrostatic terms $\quad(a . u)=.-\mathbf{7 9 0 . 2 2 0 5 3 4}$

\section{With benzaldehyde}

Optimized geometry, SCF energy, three lower frequencies, thermochemistry and PCM energy for benzaldehyde.

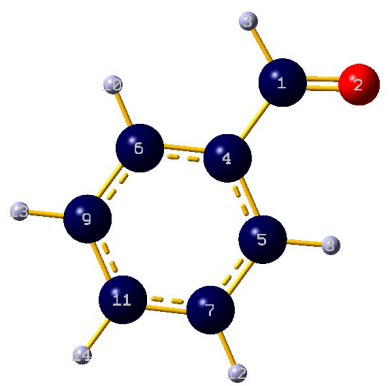

\begin{tabular}{|c|c|c|c|c|c|}
\hline Center & Atomic & Atomic & $\mathrm{COO}$ & nates (An & Eroms) \\
\hline Number & Number & Type & $\mathrm{x}$ & Y & Z \\
\hline 1 & 6 & 0 & -.049619 & -.000138 & -.022717 \\
\hline 2 & 8 & 0 & .004034 & .015552 & 1.190235 \\
\hline 3 & 1 & 0 & .882019 & -.016243 & -.632611 \\
\hline 4 & 6 & 0 & -1.299944 & .000065 & -.811043 \\
\hline 5 & 6 & 0 & -2.542495 & .019178 & -.163434 \\
\hline 6 & 6 & 0 & -1.240680 & -.019080 & -2.208637 \\
\hline 7 & 6 & 0 & -3.712792 & .019147 & -.911759 \\
\hline 8 & 1 & 0 & -2.560864 & .033696 & .922716 \\
\hline 9 & 6 & 0 & -2.414201 & -.019102 & -2.958225 \\
\hline 10 & 1 & 0 & -.271513 & -.034009 & -2.704432 \\
\hline 11 & 6 & 0 & -3.648694 & -.000004 & -2.308542 \\
\hline 12 & 1 & 0 & -4.678446 & .033984 & -.413191 \\
\hline 13 & 1 & 0 & -2.369233 & -.033945 & -4.043901 \\
\hline 14 & 1 & 0 & -4.566214 & .000005 & -2.891742 \\
\hline
\end{tabular}

$\mathrm{HF}=-345.4328128$

\begin{tabular}{|c|c|c|c|c|}
\hline & & 1 & & \\
\hline & & A & & \\
\hline Frequencies & -- & 124.3522 & & \\
\hline Red. masses & -- & 5.1678 & & \\
\hline Frc consts & -- & .0471 & & \\
\hline IR Inten & -- & 4.0439 & & \\
\hline Atom AN & $\mathrm{X}$ & $\mathrm{Y}$ & Z & $\mathrm{x}$ \\
\hline 16 & .00 & -.16 & .00 & -.01 \\
\hline 2 & .00 & .35 & .00 & .39 \\
\hline 3 & .00 & -.52 & .01 & -.19 \\
\hline 6 & .00 & -.24 & .00 & -.16 \\
\hline 6 & .00 & -.24 & .00 & -.24 \\
\hline 6 & .00 & -.10 & .00 & -.03 \\
\hline 76 & .00 & -.05 & .00 & -.14 \\
\hline
\end{tabular}

2

A

216.9526

5.6455

.1566

7.8076

Y Z

$.00-.03$

$.00-.05$

$.00-.31$

$.00 \quad .17$

$.00 \quad .04$

$.00 \quad .18$

$.00-.14$
3

A

239.4237

2.4038

.0812

7.0453

$\mathrm{X} Y \mathrm{Y}$

$\begin{array}{lll}.00 & .23 & .00\end{array}$

$\begin{array}{lll}.00 & -.06 & .00\end{array}$

$\begin{array}{lll}.01 & .83 & -.01\end{array}$

$\begin{array}{lll}.00 & -.10 & .00\end{array}$

$.00 \quad-.13 \quad .00$

$\begin{array}{lll}.00 & -.13 \quad .00\end{array}$

$.00 \quad .04 \quad .00$ 




thermodynamics:

Sum of electronic and zero-point Energies=

$-345.322342$

Sum of electronic and thermal Enthalpies=

$-345.315092$

Sum of electronic and thermal Free Energies=

Total free energy in solution:

with all non electrostatic terms

$($ a.u. $)=-345.435925$

Optimized geometry, SCF energy, three lower frequencies, thermochemistry and PCM energy for $\mathbf{T} \mathbf{S}_{\gamma}$.

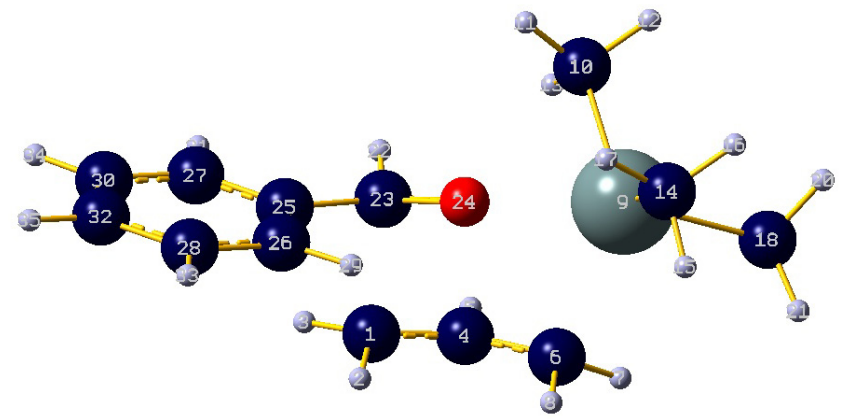

\begin{tabular}{|c|c|c|c|c|c|}
\hline \multirow{2}{*}{$\begin{array}{l}\text { Center } \\
\text { Number }\end{array}$} & \multirow{2}{*}{$\begin{array}{l}\text { Atomic } \\
\text { Number }\end{array}$} & \multirow{2}{*}{$\begin{array}{l}\text { Atomic } \\
\text { Type }\end{array}$} & \multicolumn{3}{|c|}{ Coordinates (Angstroms) } \\
\hline & & & $\mathrm{X}$ & Y & Z \\
\hline 1 & 6 & 0 & -1.054109 & 1.928212 & -0.882619 \\
\hline 2 & 1 & 0 & -1.447471 & 1.362517 & -1.723230 \\
\hline 3 & 1 & 0 & -1.748164 & 2.635412 & -0.436116 \\
\hline 4 & 6 & 0 & 0.306586 & 2.191757 & -0.840072 \\
\hline 5 & 1 & 0 & 0.653710 & 2.969889 & -0.156412 \\
\hline 6 & 6 & 0 & 1.297446 & 1.391469 & -1.457459 \\
\hline 7 & 1 & 0 & 2.272514 & 1.857363 & -1.595357 \\
\hline 8 & 1 & 0 & 0.976085 & 0.800029 & -2.316687 \\
\hline 9 & 50 & 0 & 1.982207 & -0.253244 & 0.105308 \\
\hline 10 & 6 & 0 & 1.766378 & 0.221622 & 2.199901 \\
\hline 11 & 1 & 0 & 0.911618 & -0.295301 & 2.644703 \\
\hline 12 & 1 & 0 & 2.677210 & -0.081353 & 2.727615 \\
\hline 13 & 1 & 0 & 1.642187 & 1.299584 & 2.351633 \\
\hline 14 & 6 & 0 & 1.994111 & -2.309699 & -0.523888 \\
\hline 15 & 1 & 0 & 2.105015 & -2.371489 & -1.611987 \\
\hline 16 & 1 & 0 & 2.845947 & -2.828911 & -0.071370 \\
\hline 17 & 1 & 0 & 1.066188 & -2.808762 & -0.237019 \\
\hline 18 & 6 & 0 & 4.069950 & 0.333087 & -0.248420 \\
\hline 19 & 1 & 0 & 4.250092 & 1.375512 & 0.040406 \\
\hline 20 & 1 & 0 & 4.739097 & -0.301720 & 0.345602 \\
\hline 21 & 1 & 0 & 4.344509 & 0.217462 & -1.303534 \\
\hline 22 & 1 & 0 & -0.938681 & 0.914813 & 1.323119 \\
\hline 23 & 6 & 0 & -1.146845 & 0.320047 & 0.418585 \\
\hline 24 & 8 & 0 & -0.283415 & -0.537677 & 0.039870 \\
\hline 25 & 6 & 0 & -2.597330 & 0.020732 & 0.219040 \\
\hline 26 & 6 & 0 & -2.996742 & -0.992781 & -0.659082 \\
\hline 27 & 6 & 0 & -3.566829 & 0.719519 & 0.946738 \\
\hline 28 & 6 & 0 & -4.347176 & -1.290254 & -0.815818 \\
\hline 29 & 1 & 0 & -2.232799 & -1.545340 & -1.198135 \\
\hline 30 & 6 & 0 & -4.917668 & 0.418695 & 0.792258 \\
\hline
\end{tabular}




$\begin{array}{rrrrrr}31 & 1 & 0 & -3.258835 & 1.498463 & 1.642539 \\ 32 & 6 & 0 & -5.311480 & -0.585059 & -0.093105 \\ 33 & 1 & 0 & -4.650424 & -2.080763 & -1.497947 \\ 34 & 1 & 0 & -5.663150 & 0.964145 & 1.365508 \\ 35 & 1 & 0 & -6.365459 & -0.822013 & -0.214034\end{array}$

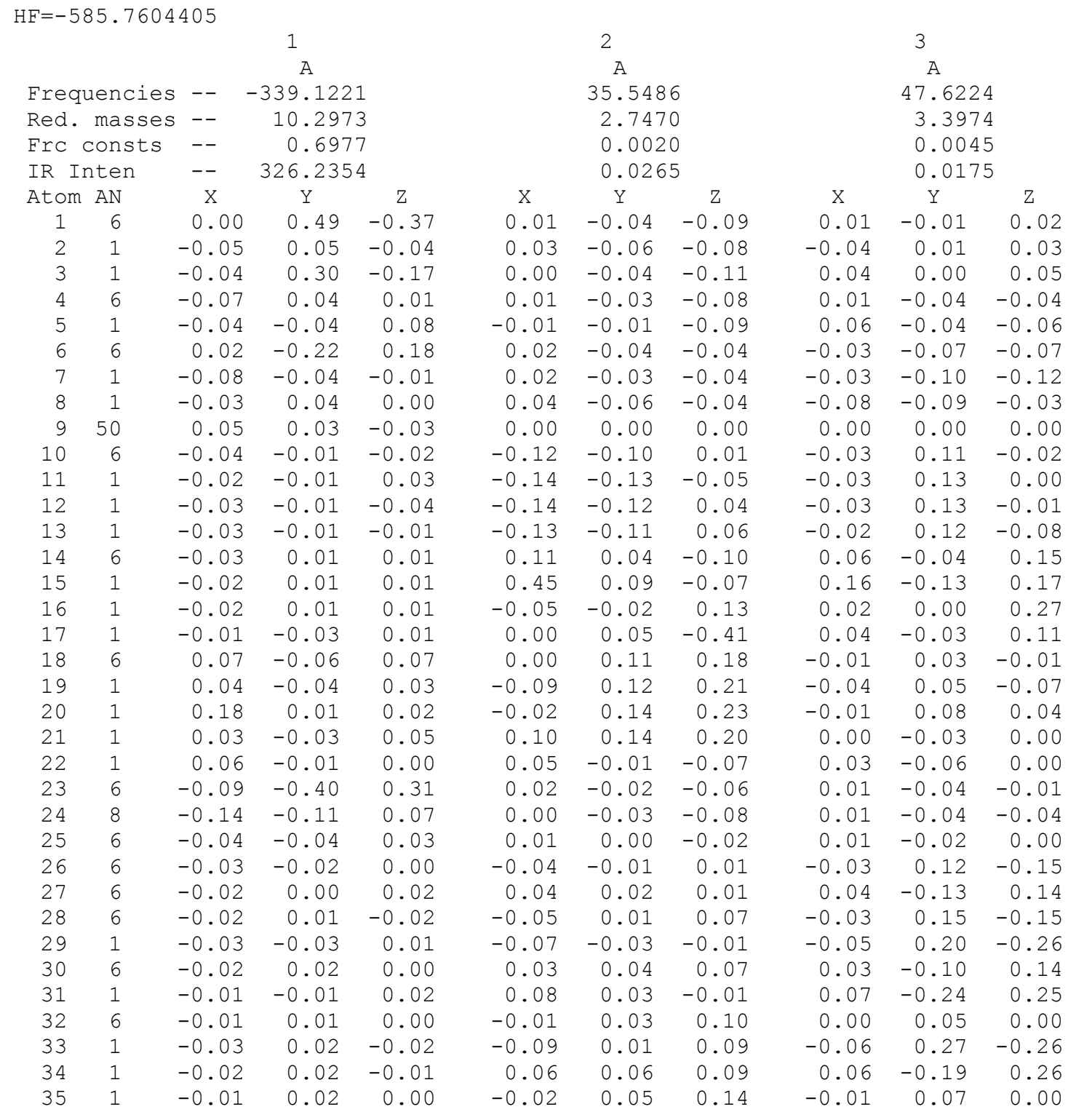

thermodynamics:

Sum of electronic and zero-point Energies=

$-585.468518$

Sum of electronic and thermal Enthalpies=

$-585.448614$

Sum of electronic and thermal Free Energies=

$-585.516660$

Total free energy in solution:

with all non electrostatic terms

$(\mathrm{a} . \mathrm{u})=$.

Optimized geometry, SCF energy, three lower frequencies, thermochemistry and PCM energy for product. 


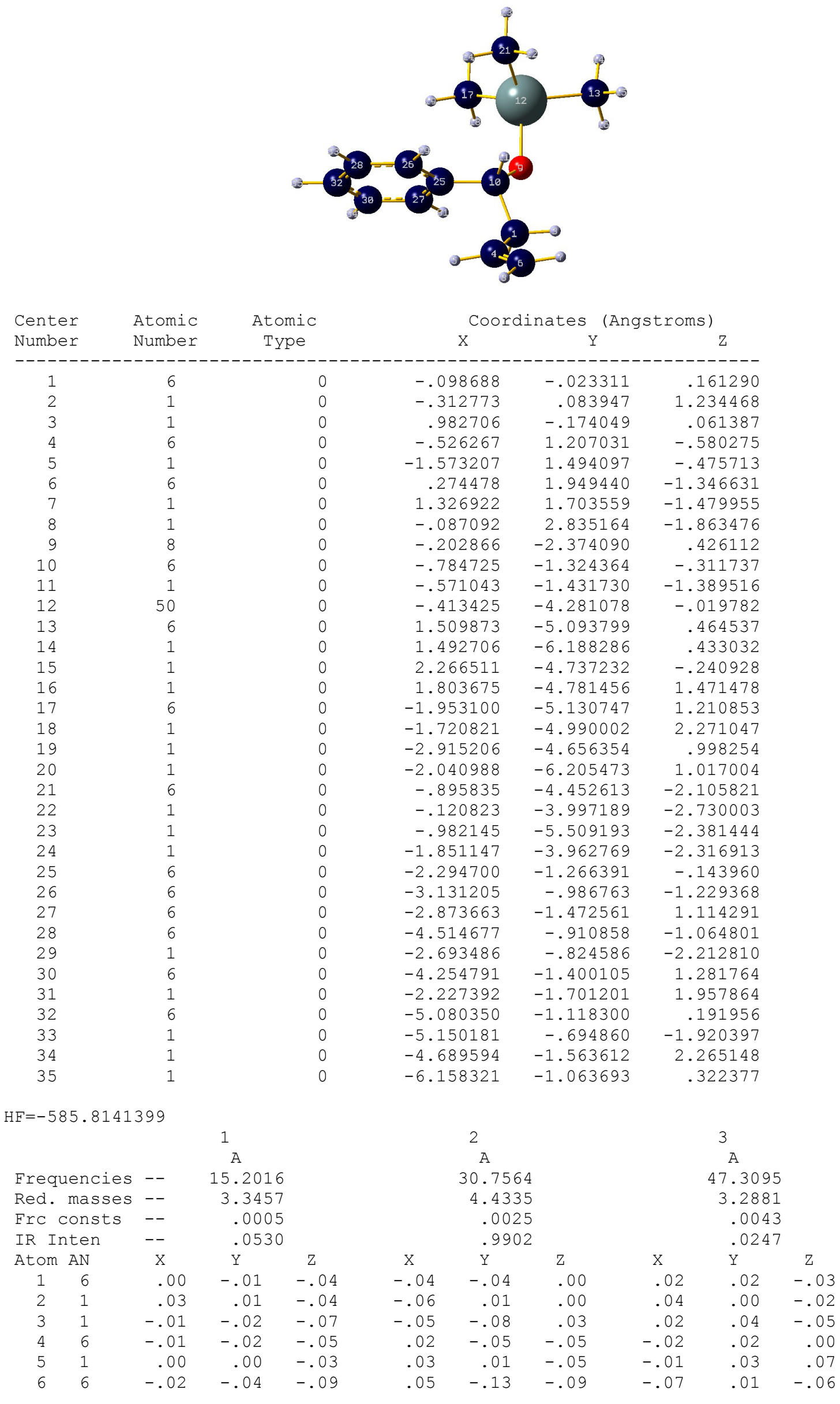




\begin{tabular}{|c|c|c|c|c|c|c|c|c|c|c|}
\hline 7 & 1 & -.03 & -.05 & -.11 & .04 & -.19 & -.08 & -.08 & .00 & -.13 \\
\hline 8 & 1 & -.03 & -.05 & -.09 & .10 & -.13 & -.13 & -.09 & .01 & -.04 \\
\hline 9 & 8 & -.02 & .00 & .00 & -.12 & -.02 & .07 & .05 & .02 & -.05 \\
\hline 10 & 6 & -.03 & -.01 & .00 & -.08 & -.03 & .03 & .03 & .01 & -.03 \\
\hline 11 & 1 & -.05 & -.03 & -.01 & -.07 & -.07 & .04 & .02 & .02 & -.03 \\
\hline 12 & 50 & .01 & .00 & -.01 & .04 & -.02 & .01 & -.01 & .01 & .01 \\
\hline 13 & 6 & -.04 & .00 & .20 & .11 & .10 & -.06 & -.04 & -.03 & .07 \\
\hline 14 & 1 & -.05 & .00 & .26 & .19 & .10 & -.06 & -.06 & -.03 & .05 \\
\hline 15 & 1 & .02 & -.06 & .24 & .06 & .16 & -.08 & -.01 & -.04 & .10 \\
\hline 16 & 1 & -.12 & .06 & .20 & .12 & .13 & -.07 & -.07 & -.05 & .08 \\
\hline 17 & 6 & -.13 & -.01 & -.19 & .12 & -.16 & .01 & -.04 & .07 & .02 \\
\hline 18 & 1 & -.24 & -.03 & -.16 & .18 & -.27 & .01 & -.15 & .28 & .01 \\
\hline 19 & 1 & -.10 & .00 & -.29 & .10 & -.14 & .11 & -.07 & -.08 & -.15 \\
\hline 20 & 1 & -.11 & -.01 & -.22 & .11 & -.14 & -.09 & .10 & .02 & .20 \\
\hline 21 & 6 & .23 & .00 & -.07 & .01 & .01 & .01 & .00 & -.03 & .01 \\
\hline 22 & 1 & .28 & .03 & .02 & -.01 & .05 & .01 & .04 & -.11 & .00 \\
\hline 23 & 1 & .30 & .00 & -.09 & .04 & .02 & -.01 & -.08 & -.04 & .05 \\
\hline 24 & 1 & .24 & -.04 & -.16 & .00 & -.01 & .04 & .03 & .03 & -.01 \\
\hline 25 & 6 & -.02 & .01 & .03 & -.08 & .02 & .01 & .03 & -.01 & -.02 \\
\hline 26 & 6 & -.05 & .00 & .05 & -.04 & .16 & .02 & .03 & .17 & .03 \\
\hline 27 & 6 & .01 & .03 & .05 & -.11 & -.05 & -.01 & .03 & -.21 & -.05 \\
\hline 28 & 6 & -.04 & .02 & .08 & -.04 & .24 & .01 & .03 & .16 & .04 \\
\hline 29 & 1 & -.07 & -.02 & .04 & -.02 & .21 & .04 & .03 & .33 & .0 \\
\hline 30 & 6 & .01 & .05 & .08 & -.11 & .02 & -.03 & .03 & -.23 & -.04 \\
\hline 31 & 1 & .03 & .03 & .04 & -.13 & -.15 & -.02 & .03 & -.34 & -.09 \\
\hline 32 & 6 & -.01 & .04 & .10 & -.07 & .17 & -.02 & .03 & -.05 & .01 \\
\hline 33 & 1 & -.06 & .01 & .10 & -.01 & .35 & .02 & .03 & .30 & .0 \\
\hline 34 & 1 & .03 & .07 & .10 & -.13 & -.03 & -.05 & .03 & -.40 & -.0 \\
\hline 35 & 1 & -.01 & .06 & .13 & -.07 & .22 & -.03 & .03 & -.07 & .0 \\
\hline
\end{tabular}

thermodynamics:

Sum of electronic and zero-point Energies=

$-585.520153$

Sum of electronic and thermal Enthalpies=

$-585.499363$

Sum of electronic and thermal Free Energies=

$-585.572210$

Total free energy in solution:

with all non electrostatic terms

$(\mathrm{a} \cdot \mathrm{u} \cdot)=$

$-585.796074$

\section{Mechanism involving a $\eta^{1}$-allyl palladium complex}

\section{$\mathbf{S} \sim \mathbf{P} \sim \boldsymbol{S}$ complex}

Optimized geometry, SCF energy, three lower frequencies, thermochemistry and PCM energy for A1.



$\begin{array}{llc}\text { Center } & \text { Atomic } & \text { Atomic } \\ \text { Number } & \text { Number } & \text { Type }\end{array}$

$\mathrm{X}_{\mathrm{Y}}^{\text {Coordinates (Angstroms) }}$ 


\begin{tabular}{|c|c|c|c|c|c|}
\hline 1 & 6 & 0 & 3.271789 & 0.447532 & 1.088251 \\
\hline 2 & 6 & 0 & 2.223705 & 0.909368 & 0.292807 \\
\hline 3 & 6 & 0 & 1.432536 & -1.724348 & 0.323265 \\
\hline 4 & 6 & 0 & 2.557924 & -1.901874 & 1.119294 \\
\hline 5 & 6 & 0 & 3.520722 & -0.906081 & 1.363119 \\
\hline 6 & 1 & 0 & 3.902263 & 1.171301 & 1.608866 \\
\hline 7 & 1 & 0 & 2.675780 & -2.836314 & 1.673286 \\
\hline 8 & 1 & 0 & 4.379535 & -1.157534 & 1.977569 \\
\hline 9 & 15 & 0 & -0.067927 & -2.655846 & 0.446161 \\
\hline 10 & 1 & 0 & -0.529665 & -2.698740 & 1.785329 \\
\hline 11 & 1 & 0 & 0.188001 & -4.031160 & 0.233451 \\
\hline 12 & 15 & 0 & 1.541161 & 2.529764 & 0.354332 \\
\hline 13 & 1 & 0 & 1.869784 & 3.111565 & 1.602576 \\
\hline 14 & 1 & 0 & 2.220926 & 3.427384 & -0.506031 \\
\hline 15 & 16 & 0 & -0.452517 & 2.625682 & -0.051855 \\
\hline 16 & 16 & 0 & -1.502217 & -2.022739 & -0.834290 \\
\hline 17 & 46 & 0 & -1.011332 & 0.333094 & -0.541493 \\
\hline 18 & 6 & 0 & -3.016936 & 0.748371 & -0.124372 \\
\hline 19 & 1 & 0 & -3.201976 & 1.750886 & -0.525354 \\
\hline 20 & 1 & 0 & -3.618560 & 0.007097 & -0.658486 \\
\hline 21 & 6 & 0 & -3.164010 & 0.671092 & 1.335982 \\
\hline 22 & 1 & 0 & -2.777381 & 1.522744 & 1.898606 \\
\hline 23 & 6 & 0 & -3.704520 & -0.351967 & 2.021335 \\
\hline 24 & 1 & 0 & -4.106220 & -1.226867 & 1.513751 \\
\hline 25 & 1 & 0 & -3.768188 & -0.339465 & 3.106661 \\
\hline 26 & 15 & 0 & 1.262305 & -0.248909 & -0.687933 \\
\hline 27 & 6 & 0 & 2.248904 & -0.578041 & -2.210250 \\
\hline 28 & 1 & 0 & 3.267586 & -0.890185 & -1.958551 \\
\hline 29 & 1 & 0 & 1.755461 & -1.368648 & -2.785902 \\
\hline 30 & 1 & 0 & 2.285882 & 0.329052 & -2.823774 \\
\hline
\end{tabular}

$\mathrm{HF}=-2298.8263281$

\begin{tabular}{|c|c|c|c|c|}
\hline \multicolumn{5}{|c|}{ \pm} \\
\hline \multicolumn{2}{|c|}{ Frequencies } & $5--$ & 18.506 & \\
\hline Red. & masses & $5--$ & 4.595 & \\
\hline Frc & consts & -- & 0.000 & \\
\hline IR I & nten & -- & 0.754 & \\
\hline Atom & AN & $\mathrm{X}$ & $\mathrm{Y}$ & $\mathrm{Z}$ \\
\hline 1 & 6 & 0.00 & -0.01 & 0.02 \\
\hline 2 & 6 & -0.03 & -0.01 & 0.05 \\
\hline 3 & 6 & 0.05 & -0.03 & -0.05 \\
\hline 4 & 6 & 0.07 & -0.03 & -0.07 \\
\hline 5 & 6 & 0.04 & -0.01 & -0.04 \\
\hline 6 & 1 & -0.02 & -0.01 & 0.0 \\
\hline 7 & 1 & 0.11 & -0.05 & -0.1 \\
\hline 8 & 1 & 0.05 & -0.01 & -0 \\
\hline 9 & 15 & 0.10 & -0.11 & -0.0 \\
\hline 10 & 1 & 0.20 & -0.31 & -0.0 \\
\hline 11 & 1 & 0.13 & -0.08 & -0.2 \\
\hline 12 & 15 & -0.04 & -0.01 & 0. \\
\hline 13 & 1 & -0.18 & -0.10 & 0.1 \\
\hline 14 & 1 & 0.04 & 0.06 & 0.24 \\
\hline 15 & 16 & 0.00 & 0.01 & -0.1 \\
\hline 16 & 16 & -0.01 & -0.02 & 0.0 \\
\hline 17 & 46 & -0.01 & -0.02 & 0.01 \\
\hline 18 & 6 & -0.02 & 0.01 & -0.05 \\
\hline 19 & 1 & -0.01 & -0.03 & -0.17 \\
\hline 20 & 1 & -0.01 & -0.05 & 0.02 \\
\hline 21 & 6 & -0.05 & 0.18 & -0.05 \\
\hline 22 & 1 & -0.07 & 0.24 & -0.14 \\
\hline 23 & 6 & -0.07 & 0.25 & 0.05 \\
\hline 24 & 1 & -0.05 & 0.19 & 0.14 \\
\hline 25 & 1 & -0.09 & 0.37 & 0.05 \\
\hline 26 & 15 & -0.01 & 0.01 & 0.02 \\
\hline 27 & 6 & -0.02 & 0.10 & -0.01 \\
\hline 28 & 1 & 0.00 & 0.14 & -0.04 \\
\hline
\end{tabular}

\section{2}

35.8712

5.0859

0.0039

2.0220

$\mathrm{X}$

$-0.07$

$\mathrm{Y}$

$-0.04$

$-0.04$

$-0.07$

$-0.07$

$-0.10$

$-0.09$

$-0.09$

$-0.05$

$-0.09$

$-0.05$

$-0.05$

$-0.19$

0.04

0.00

$-0.01$

$-0.01$

0.02

$-0.04$

$-0.02$

0.19

0.22

0.31

0.28

0.44

0.00

0.08

0.07
$-0.01$

0.00

0.00

$-0.01$

$-0.01$

$-0.01$

$-0.01$

$-0.01$

0.02

0.05

0.01

0.00

$-0.08$

0.05

0.02

0.00

$-0.01$

$-0.03$

$-0.05$

$-0.06$

0.00

0.03

0.00

$-0.03$

0.02

0.01

0.02

0.02
3

A 46.7377

4.3188 0.0056 3.7143 Y Z $\mathrm{X}$
-0.08 $0.03 \quad 0.10$
0.00 0.08 0.05 0.00 0.03

0.07

0.12

0.03

0.10

$-0.03$

$-0.04$

0.01

0.07

0.14

0.20

$-0.16$

$-0.08$

$-0.03$

0.09

0.09

0.17

0.11

0.04

0.21

0.28

0.21

$-0.01$

0.04

0.10
$-0.01$

0.00

$-0.06$

$-0.10$

$-0.12$

$-0.09$

$-0.16$

0.05

0.08

0.08

0.03

0.22

$-0.07$

$-0.03$

0.01

0.00

0.01

0.00

0.01

0.02

0.03

0.02

0.02

0.03

0.01

0.01
$0.00 \quad-0.01$

$\begin{array}{rr}-0.01 & 0.00\end{array}$

$0.03 \quad 0.10$

$0.05 \quad 0.17$

$0.06 \quad 0.12$

$0.04 \quad 0.12$

$0.07 \quad 0.26$

$-0.10-0.13$

$-0.32-0.13$

$-0.06-0.33$

$0.02-0.16$

$0.19-0.29$

$-0.13-0.40$

$\begin{array}{ll}-0.01 & 0.11\end{array}$

$0.01 \quad-0.04$

$0.00 \quad 0.01$

$0.00 \quad 0.03$

$\begin{array}{ll}-0.03 & -0.02\end{array}$

$\begin{array}{ll}-0.03 & 0.08\end{array}$

$0.07 \quad 0.04$

0.090 .00

$0.10 \quad 0.09$

$0.08 \quad 0.14$

$\begin{array}{rr}0.15 & 0.09\end{array}$

$\begin{array}{ll}-0.01-0.01 & -0.01\end{array}$

$\begin{array}{ll}-0.01 & -0.01\end{array}$

$\begin{array}{ll}-0.02 & 0.00\end{array}$ 


\begin{tabular}{|c|c|c|c|c|c|c|}
\hline $29 \quad 1$ & 01 & 11 & -0.03 & 0.12 & 0.01 & 0.02 \\
\hline $0 \quad 1$ & -0.07 & 0.13 & 0.03 & 0.11 & 0.02 & 0.05 \\
\hline
\end{tabular}

thermodynamics:

Sum of electronic and zero-point Energies=

$-2298.609658$

Sum of electronic and thermal Enthalpies=

$-2298.589308$

Sum of electronic and thermal Free Energies=

$-2298.659191$

Total free energy in solution:

with all non electrostatic terms

$($ a.u. $)=-2298.826569$

Optimized geometry, SCF energy, three lower frequencies, thermochemistry and PCM energy for $\mathbf{T S}_{\mathrm{A1A2}}$.

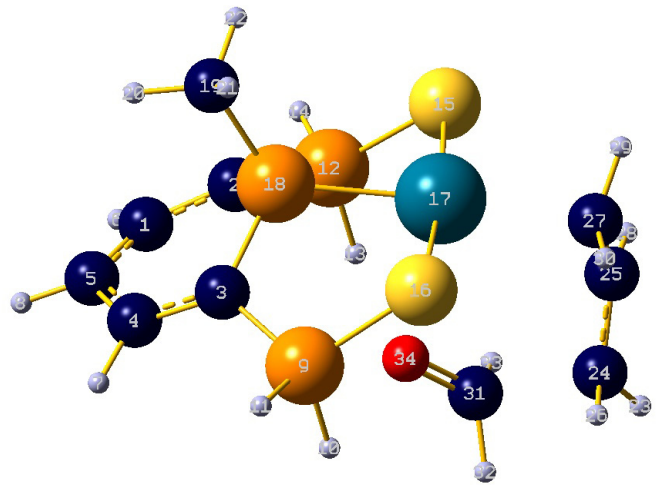

\begin{tabular}{|c|c|c|c|c|c|}
\hline \multirow{2}{*}{$\begin{array}{l}\text { Center } \\
\text { Number }\end{array}$} & \multirow{2}{*}{$\begin{array}{l}\text { Atomic } \\
\text { Number }\end{array}$} & \multirow{2}{*}{$\begin{array}{c}\text { Atomic } \\
\text { Type }\end{array}$} & \multicolumn{3}{|c|}{ Coordinates (Angstroms) } \\
\hline & & & $\mathrm{X}$ & Y & Z \\
\hline 1 & 6 & 0 & -3.091638 & -1.390075 & .932802 \\
\hline 2 & 6 & 0 & -1.905931 & -1.457429 & .203894 \\
\hline 3 & 6 & 0 & -2.034080 & 1.282132 & .288806 \\
\hline 4 & 6 & 0 & -3.204552 & 1.057577 & 1.006811 \\
\hline 5 & 6 & 0 & -3.810046 & -.203692 & 1.157077 \\
\hline 6 & 1 & 0 & -3.452975 & -2.285183 & 1.444514 \\
\hline 7 & 1 & 0 & -3.648431 & 1.881041 & 1.571057 \\
\hline 8 & 1 & 0 & -4.754745 & -.263210 & 1.688839 \\
\hline 9 & 15 & 0 & -.833040 & 2.532336 & .609093 \\
\hline 10 & 1 & 0 & -.372520 & 2.509077 & 1.940206 \\
\hline 11 & 1 & 0 & -1.432418 & 3.811635 & .536930 \\
\hline 12 & 15 & 0 & -.570037 & -2.566426 & .493042 \\
\hline 13 & 1 & 0 & -.093810 & -2.496395 & 1.819561 \\
\hline 14 & 1 & 0 & -1.019670 & -3.906088 & .436703 \\
\hline 15 & 16 & 0 & .927912 & -2.330440 & -.861648 \\
\hline 16 & 16 & 0 & .681835 & 2.464823 & -.746059 \\
\hline 17 & 46 & 0 & .910745 & .079062 & -.792937 \\
\hline 18 & 15 & 0 & -1.397980 & -.030307 & -.749772 \\
\hline 19 & 6 & 0 & -2.425911 & -.034285 & -2.271080 \\
\hline 20 & 1 & 0 & -3.488506 & -.093109 & -2.014175 \\
\hline 21 & 1 & 0 & -2.233533 & .884604 & -2.834370 \\
\hline 22 & 1 & 0 & -2.147510 & -.896916 & -2.885142 \\
\hline 23 & 1 & 0 & 3.991085 & -.198582 & 2.516102 \\
\hline 24 & 6 & 0 & 3.366383 & .252555 & 1.745170 \\
\hline 25 & 6 & 0 & 3.413322 & -.349598 & .478087 \\
\hline 26 & 1 & 0 & 3.345921 & 1.342539 & 1.767625 \\
\hline 27 & 6 & 0 & 3.084511 & .260977 & -.751772 \\
\hline 28 & 1 & 0 & 3.600622 & -1.424972 & .455352 \\
\hline 29 & 1 & 0 & 3.361104 & -.285461 & -1.655561 \\
\hline 30 & 1 & 0 & 3.236555 & 1.338130 & -.816346 \\
\hline 31 & 6 & 0 & 1.680940 & -.207972 & 2.533658 \\
\hline 32 & 1 & 0 & 1.907535 & .362518 & 3.458795 \\
\hline 33 & 1 & 0 & 1.904109 & -1.288164 & 2.675827 \\
\hline
\end{tabular}


$\mathrm{HF}=-2413.2674159$

\begin{tabular}{|c|c|c|c|c|c|c|c|c|c|c|}
\hline & & & 1 & & & 2 & & & 3 & \\
\hline & & & A & & & A & & & A & \\
\hline Frequ & dencies & -- & -330.978 & & & 34.54 & & & 52.060 & \\
\hline Red. & masses & -- & 9.602 & & & 4.30 & & & 5.400 & \\
\hline Frc & consts & -- & .619 & & & .00 & & & .008 & \\
\hline IR In & nten & -- & 146.027 & & & 1.08 & & & 1.844 & \\
\hline Atom & AN & $\mathrm{x}$ & Y & Z & $\mathrm{X}$ & Y & Z & $\mathrm{X}$ & Y & Z \\
\hline 1 & 6 & -.01 & -.01 & .01 & .02 & -.02 & .01 & .12 & .02 & .15 \\
\hline 2 & 6 & -.01 & .00 & .02 & .00 & -.02 & -.03 & .07 & .02 & .07 \\
\hline 3 & 6 & -.01 & -.01 & .01 & .05 & -.01 & .06 & .01 & .03 & -.03 \\
\hline 4 & 6 & -.01 & .00 & .01 & .07 & -.02 & .08 & .05 & .02 & .05 \\
\hline 5 & 6 & -.01 & .00 & .01 & .05 & -.01 & .06 & .10 & .01 & .15 \\
\hline 6 & 1 & -.02 & .00 & .01 & .02 & -.03 & -.01 & .18 & .04 & .21 \\
\hline 7 & 1 & -.02 & .00 & .01 & .10 & -.03 & .12 & .06 & .03 & .04 \\
\hline 8 & 1 & -.01 & .00 & .00 & .06 & -.01 & .09 & .14 & .01 & .21 \\
\hline 9 & 15 & -.02 & .02 & -.03 & .05 & -.02 & .05 & .00 & .05 & -.08 \\
\hline 10 & 1 & -.04 & .07 & -.01 & .02 & .03 & .06 & -.02 & .12 & -.07 \\
\hline 11 & 1 & -.03 & .01 & .00 & .06 & -.01 & -.01 & .00 & .05 & -.13 \\
\hline 12 & 15 & -.02 & -.02 & -.03 & -.01 & -.03 & -.06 & .09 & .03 & .01 \\
\hline 13 & 1 & -.05 & -.10 & -.01 & .01 & -.05 & -.07 & .14 & -.01 & -.01 \\
\hline 14 & 1 & -.04 & -.02 & -.03 & -.02 & -.03 & -.07 & .09 & .03 & .00 \\
\hline 15 & 16 & .02 & -.01 & .02 & -.01 & -.03 & -.07 & .02 & .03 & -.06 \\
\hline 16 & 16 & .01 & .01 & .02 & .06 & -.04 & .05 & .02 & .02 & -.05 \\
\hline 17 & 46 & .01 & .01 & -.03 & .01 & -.04 & -.03 & .01 & .02 & -.04 \\
\hline 18 & 15 & -.02 & .00 & .00 & .00 & .00 & .00 & .00 & .00 & .00 \\
\hline 19 & 6 & -.04 & .00 & .01 & -.04 & .08 & .03 & -.09 & -.10 & .05 \\
\hline 20 & 1 & -.04 & .00 & -.02 & -.03 & .09 & .06 & -.07 & -.13 & .12 \\
\hline 21 & 1 & -.02 & .00 & .01 & -.03 & .09 & .06 & -.16 & -.12 & .00 \\
\hline 22 & 1 & -.02 & .00 & .01 & -.08 & .09 & .00 & -.09 & -.11 & .08 \\
\hline 23 & 1 & .05 & -.11 & .13 & -.12 & .33 & .09 & -.20 & -.18 & .13 \\
\hline 24 & 6 & .60 & .09 & -.17 & -.11 & .22 & .01 & -.14 & -.13 & .12 \\
\hline 25 & 6 & .15 & .01 & .06 & -.06 & .06 & .09 & -.08 & -.06 & .09 \\
\hline 26 & 1 & .26 & .06 & .03 & -.14 & .22 & -.12 & -.13 & -.13 & .18 \\
\hline 27 & 6 & -.22 & -.04 & -.01 & .01 & -.09 & -.01 & .00 & .01 & .10 \\
\hline 28 & 1 & .20 & .02 & .06 & -.06 & .06 & .23 & -.08 & -.06 & .04 \\
\hline 29 & 1 & -.03 & -.06 & .05 & .04 & -.22 & .08 & .06 & .06 & .09 \\
\hline 30 & 1 & .07 & -.08 & -.06 & .03 & -.10 & -.14 & .02 & .02 & .17 \\
\hline 31 & 6 & -.42 & -.09 & .24 & -.11 & .24 & .04 & -.20 & -.14 & .01 \\
\hline 32 & 1 & .09 & .19 & -.09 & -.17 & .39 & -.04 & -.26 & -.15 & .03 \\
\hline 33 & 1 & -.05 & -.01 & -.02 & -.04 & .27 & .21 & -.20 & -.14 & 01 \\
\hline 34 & 8 & -.09 & -.02 & .14 & -.12 & .06 & -.04 & -.16 & -.14 & -.05 \\
\hline
\end{tabular}

thermodynamics:

Sum of electronic and zero-point Energies=

$-2413.019965$

Sum of electronic and thermal Enthalpies=

$-2412.997781$

Sum of electronic and thermal Free Energies=

$-2413.069810$

Total free energy in solution:

with all non electrostatic terms

(a.u.) $=-2413.266563$

Optimized geometry, SCF energy, three lower frequencies, thermochemistry and PCM energy for A2. 







$\begin{array}{rrrrrrrrrrr}9 & 15 & 0.11 & 0.02 & 0.10 & 0.02 & 0.00 & -0.01 & -0.01 & -0.03 & -0.10 \\ 10 & 1 & 0.10 & -0.06 & 0.23 & -0.02 & 0.01 & 0.01 & 0.05 & 0.06 & -0.30 \\ 11 & 1 & 0.24 & 0.12 & 0.14 & 0.06 & -0.01 & -0.01 & -0.17 & -0.18 & -0.16 \\ 12 & 15 & -0.11 & 0.04 & -0.09 & 0.05 & 0.01 & -0.04 & 0.00 & -0.01 & 0.01 \\ 13 & 1 & -0.14 & -0.03 & -0.21 & 0.03 & -0.01 & -0.07 & -0.04 & 0.02 & 0.12 \\ 14 & 1 & -0.21 & 0.16 & -0.12 & 0.07 & 0.05 & -0.06 & 0.11 & -0.08 & 0.02 \\ 15 & 16 & -0.03 & 0.05 & 0.04 & 0.02 & -0.02 & -0.06 & -0.09 & 0.04 & -0.16 \\ 16 & 16 & 0.03 & 0.03 & -0.07 & -0.01 & -0.02 & -0.08 & 0.10 & 0.05 & 0.15 \\ 17 & 46 & 0.00 & 0.05 & -0.01 & -0.01 & -0.01 & -0.06 & -0.01 & 0.03 & -0.02 \\ 18 & 6 & 0.00 & -0.05 & -0.01 & -0.03 & 0.07 & 0.05 & -0.03 & -0.12 & 0.08 \\ 19 & 1 & -0.04 & -0.01 & -0.10 & 0.05 & 0.13 & -0.02 & -0.12 & -0.21 & 0.21 \\ 20 & 1 & 0.04 & 0.00 & 0.07 & -0.05 & 0.15 & 0.14 & 0.09 & -0.24 & -0.03 \\ 21 & 6 & -0.01 & -0.21 & 0.00 & -0.10 & 0.02 & 0.10 & -0.06 & -0.06 & 0.10 \\ 22 & 1 & -0.06 & -0.27 & -0.08 & -0.07 & -0.07 & 0.01 & -0.19 & 0.05 & 0.22 \\ 23 & 6 & 0.03 & -0.28 & 0.09 & -0.20 & 0.07 & 0.26 & 0.06 & -0.14 & -0.01 \\ 24 & 1 & 0.07 & -0.22 & 0.17 & -0.24 & 0.16 & 0.36 & 0.19 & -0.26 & -0.12 \\ 25 & 1 & 0.02 & -0.39 & 0.09 & -0.25 & 0.03 & 0.29 & 0.03 & -0.10 & 0.02 \\ 26 & 8 & 0.00 & 0.10 & -0.03 & -0.01 & 0.00 & -0.05 & 0.00 & 0.02 & 0.01 \\ 27 & 6 & 0.00 & -0.02 & -0.01 & -0.06 & -0.05 & -0.01 & -0.02 & 0.10 & 0.02 \\ 28 & 1 & 0.03 & -0.06 & 0.06 & -0.13 & -0.10 & 0.06 & 0.06 & 0.19 & -0.12 \\ 29 & 1 & -0.04 & -0.07 & -0.08 & -0.04 & -0.11 & -0.08 & -0.13 & 0.23 & 0.13 \\ 30 & 15 & 0.00 & -0.02 & 0.01 & -0.01 & 0.00 & 0.01 & -0.01 & 0.00 & -0.01 \\ 31 & 6 & -0.01 & -0.12 & 0.01 & -0.08 & 0.01 & 0.06 & -0.04 & 0.04 & 0.01 \\ 32 & 1 & -0.02 & -0.16 & 0.01 & -0.07 & 0.02 & 0.11 & -0.03 & 0.05 & 0.03 \\ 33 & 1 & -0.05 & -0.12 & -0.02 & -0.11 & 0.00 & 0.04 & -0.04 & 0.05 & 0.02 \\ 34 & 1 & 0.03 & -0.13 & 0.04 & -0.12 & 0.01 & 0.05 & -0.06 & 0.05 & -0.01\end{array}$

thermodynamics:

Sum of electronic and zero-point Energies=

$-2413.056661$

Sum of electronic and thermal Enthalpies=

$-2413.033691$

Sum of electronic and thermal Free Energies=

$-2413.111147$

Total free energy in solution:

with all non electrostatic terms

$($ a.u. $)=-2413.313886$

Optimized geometry, SCF energy, three lower frequencies, thermochemistry and PCM energy for $\mathbf{T S}_{\mathbf{A 2 A 1}}$.

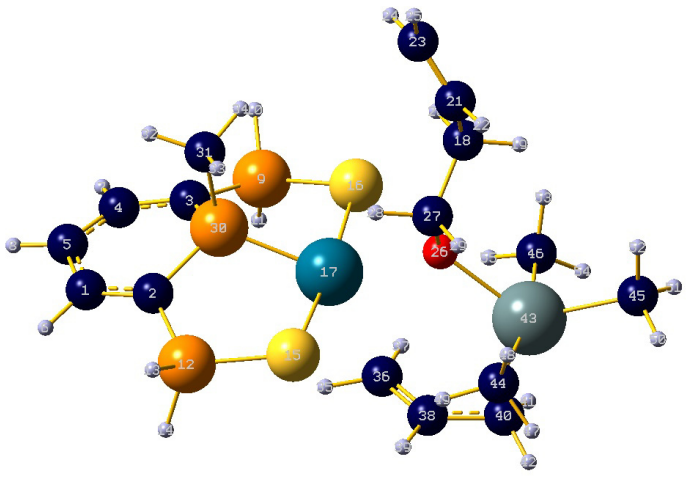

\begin{tabular}{|c|c|c|c|c|c|}
\hline \multirow{2}{*}{$\begin{array}{l}\text { Center } \\
\text { Number }\end{array}$} & \multirow{2}{*}{$\begin{array}{l}\text { Atomic } \\
\text { Number }\end{array}$} & \multirow{2}{*}{$\begin{array}{c}\text { Atomic } \\
\text { Type }\end{array}$} & \multicolumn{3}{|c|}{ Coordinates (Angstroms) } \\
\hline & & & $x$ & $\mathrm{Y}$ & Z \\
\hline 1 & 6 & 0 & 5.021768 & -0.697468 & -1.108278 \\
\hline 2 & 6 & 0 & 3.666912 & -0.455829 & -1.324760 \\
\hline 3 & 6 & 0 & 3.677938 & 0.223203 & 1.351057 \\
\hline 4 & 6 & 0 & 5.028773 & -0.098564 & 1.271226 \\
\hline 5 & 6 & 0 & 5.705772 & -0.410725 & 0.081824 \\
\hline 6 & 1 & 0 & 5.591153 & -1.215209 & -1.882526 \\
\hline 7 & 1 & 0 & 5.600738 & -0.193564 & 2.196224 \\
\hline 8 & 1 & 0 & 6.767955 & -0.625967 & 0.132419 \\
\hline 9 & 15 & 0 & 2.658670 & 0.154356 & 2.787067 \\
\hline 10 & 1 & 0 & 3.084496 & 1.065196 & 3.780396 \\
\hline
\end{tabular}




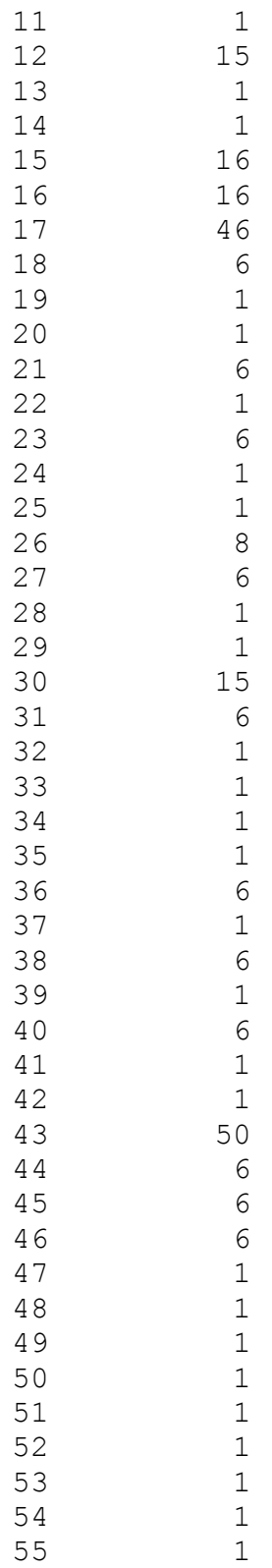

$\begin{array}{rrr}2.861608 & -1.068942 & 3.474322 \\ 2.662008 & -1.199369 & -2.563394 \\ 2.994190 & -0.754332 & -3.863980 \\ 2.994509 & -2.571872 & -2.689410 \\ 0.684544 & -0.853834 & -2.241028 \\ 0.714891 & 0.533362 & 2.331942 \\ 0.610876 & -0.190582 & 0.061384 \\ -1.953782 & 3.081207 & -0.158817 \\ -2.934669 & 2.923092 & 0.314381 \\ -1.224017 & 3.193164 & 0.652799 \\ -1.990560 & 4.314358 & -1.009156 \\ -2.724282 & 4.315202 & -1.818532 \\ -1.196660 & 5.377339 & -0.865984 \\ -0.453133 & 5.429115 & -0.071765 \\ -1.265970 & 6.239789 & -1.524844 \\ -1.577753 & 0.674353 & -0.145173 \\ -1.608623 & 1.816852 & -0.963535 \\ -0.626981 & 1.963267 & -1.440725 \\ -2.334301 & 1.700107 & -1.786531 \\ 2.723337 & 0.508275 & -0.141834 \\ 2.934349 & 2.276969 & -0.588919 \\ 3.997907 & 2.530042 & -0.660389 \\ 2.442975 & 2.464230 & -1.549444 \\ 2.455265 & 2.897202 & 0.175607 \\ 0.711484 & -2.797380 & 0.434142 \\ -0.286478 & -2.608859 & 0.823976 \\ -0.371046 & -2.463057 & 1.898406 \\ -1.385572 & -2.893701 & 0.057384 \\ -1.197088 & -3.082485 & -1.001329 \\ -2.769539 & -2.865338 & 0.453036 \\ -2.961772 & -3.039763 & 1.513012 \\ -3.437671 & -3.431214 & -0.198365 \\ -3.273866 & -0.596842 & 0.142607 \\ -3.262204 & -0.927458 & -2.036590 \\ -5.336502 & 0.150616 & 0.237004 \\ -2.836026 & -0.315766 & 2.279032 \\ -3.994722 & -1.711813 & -2.265499 \\ -3.557570 & -0.026874 & -2.587502 \\ -2.280755 & -1.249963 & -2.399680 \\ -5.958315 & -0.288257 & -0.551204 \\ -5.793893 & -0.057247 & 1.210615 \\ -5.343530 & 1.239464 & 0.095568 \\ -2.882824 & 0.750725 & 2.524914 \\ -3.601894 & -0.845665 & 2.859340 \\ -1.851415 & -0.695311 & 2.563739\end{array}$

$\mathrm{HF}=-2653.6098931$

\begin{tabular}{|c|c|c|c|c|}
\hline & & & 1 & \\
\hline & & & A & \\
\hline Freq & uencies & -- & -129.0596 & \\
\hline Red. & masses & -- & 10.6508 & \\
\hline Frc & consts & -- & 0.1045 & \\
\hline IR I & nten & -- & 72.7484 & \\
\hline Atom & AN & $\mathrm{x}$ & $\mathrm{Y}$ & Z \\
\hline 1 & 6 & 0.03 & -0.04 & 0.01 \\
\hline 2 & 6 & 0.02 & -0.08 & 0.02 \\
\hline 3 & 6 & 0.02 & -0.08 & 0.03 \\
\hline 4 & 6 & 0.03 & -0.04 & 0.02 \\
\hline 5 & 6 & 0.02 & -0.03 & 0.01 \\
\hline 6 & 1 & 0.05 & -0.01 & 0.00 \\
\hline 7 & 1 & 0.05 & -0.01 & 0.01 \\
\hline 8 & 1 & 0.03 & 0.00 & 0.01 \\
\hline 9 & 15 & 0.00 & 0.00 & 0.02 \\
\hline 10 & 1 & 0.03 & 0.01 & -0.01 \\
\hline 11 & 1 & -0.07 & 0.01 & 0.06 \\
\hline 12 & 15 & 0.01 & -0.01 & -0.01 \\
\hline 13 & 1 & 0.05 & 0.02 & 0.01 \\
\hline 14 & 1 & -0.06 & -0.02 & -0.05 \\
\hline
\end{tabular}

2

A

17.8016

4.3887

0.0008

0.2135

$\mathrm{X}$

$-0.01$

$-0.02$

$-0.01$

$-0.01$

$-0.01$

$-0.01$

0.00

$-0.01$

0.00

0.00

$-0.01$

$-0.01$

$-0.05$

0.04

\begin{tabular}{cr}
$Y$ & \multicolumn{1}{c}{$Z$} \\
0.09 & -0.03 \\
0.08 & -0.02 \\
0.01 & 0.00 \\
0.03 & -0.01 \\
0.07 & -0.03 \\
0.12 & -0.05 \\
0.01 & -0.02 \\
0.08 & -0.04 \\
-0.04 & 0.00 \\
-0.08 & 0.03 \\
-0.07 & -0.04 \\
0.09 & -0.03 \\
0.16 & -0.02 \\
0.11 & -0.09
\end{tabular}

$0.11-0.09$
3 A 19.2228 5.0718 0.0011 0.0845 $\mathrm{X}$

0.01

0.00

$-0.01$

0.00

0.00

0.02

0.00

0.01

0.00

$-0.04$

0.06

0.01

0.03

$-0.01$ $\begin{array}{lll}\text { Y } & \mathrm{Z}\end{array}$

$0.00 \quad 0.02$

$\begin{array}{ll}-0.03 & 0.01\end{array}$

$0.09-0.03$

$\begin{array}{ll}0.12 & -0.01\end{array}$

$\begin{array}{ll}0.08 & 0.00\end{array}$

$\begin{array}{ll}-0.02 & 0.04\end{array}$

$0.18-0.01$

$\begin{array}{lr}0.10 & 0.01\end{array}$

$\begin{array}{ll}0.14 & -0.02\end{array}$

$0.22-0.07$

0.190 .06

$\begin{array}{rr}0.11 & 0.04\end{array}$

$\begin{array}{ll}-0.20 & 0.02\end{array}$

$\begin{array}{ll}-0.12 & 0.14\end{array}$ 


\begin{tabular}{|c|c|c|c|c|c|c|c|c|c|c|}
\hline 15 & 16 & 0.01 & 0.07 & -0.01 & -0.02 & 0.01 & 0.01 & 0.01 & -0.06 & -0.01 \\
\hline 16 & 16 & 0.02 & 0.07 & -0.03 & 0.00 & -0.02 & 0.02 & -0.02 & 0.03 & -0.03 \\
\hline 17 & 46 & -0.03 & 0.18 & -0.06 & -0.02 & 0.00 & 0.01 & 0.00 & -0.02 & -0.01 \\
\hline 18 & 6 & 0.06 & -0.08 & 0.03 & 0.17 & 0.00 & -0.03 & 0.09 & -0.01 & 0.13 \\
\hline 19 & 1 & 0.07 & -0.12 & 0.03 & 0.20 & 0.09 & 0.07 & 0.09 & 0.01 & 0.15 \\
\hline 20 & 1 & 0.05 & -0.05 & 0.03 & 0.26 & -0.05 & -0.10 & 0.11 & -0.09 & 0.12 \\
\hline 21 & 6 & 0.01 & -0.08 & 0.03 & 0.20 & -0.01 & -0.04 & 0.13 & 0.04 & 0.20 \\
\hline 22 & 1 & 0.01 & -0.11 & 0.04 & 0.12 & 0.04 & 0.03 & 0.11 & 0.11 & 0.22 \\
\hline 23 & 6 & -0.02 & -0.05 & 0.02 & 0.32 & -0.09 & -0.15 & 0.17 & -0.01 & 0.25 \\
\hline 24 & 1 & -0.02 & -0.03 & 0.02 & 0.41 & -0.14 & -0.22 & 0.19 & -0.08 & 0.24 \\
\hline 25 & 1 & -0.06 & -0.06 & 0.03 & 0.34 & -0.09 & -0.16 & 0.20 & 0.04 & 0.30 \\
\hline 26 & 8 & 0.17 & -0.09 & -0.01 & -0.02 & -0.03 & -0.02 & 0.00 & -0.03 & 0.00 \\
\hline 27 & 6 & 0.12 & -0.06 & 0.02 & -0.02 & -0.04 & -0.04 & 0.01 & 0.01 & 0.05 \\
\hline 28 & 1 & 0.11 & 0.00 & 0.01 & -0.06 & -0.13 & -0.15 & 0.00 & 0.00 & 0.02 \\
\hline 29 & 1 & 0.12 & -0.10 & 0.03 & -0.11 & 0.00 & 0.04 & -0.03 & 0.08 & 0.08 \\
\hline 30 & 15 & 0.02 & -0.13 & 0.04 & -0.02 & 0.03 & 0.02 & -0.01 & 0.01 & -0.04 \\
\hline 31 & 6 & -0.09 & -0.12 & 0.03 & -0.05 & 0.05 & 0.06 & -0.04 & -0.01 & -0.13 \\
\hline 32 & 1 & -0.10 & -0.08 & 0.04 & -0.06 & 0.06 & 0.06 & -0.05 & 0.00 & -0.15 \\
\hline 33 & 1 & -0.10 & -0.14 & 0.03 & -0.06 & 0.07 & 0.07 & -0.05 & -0.07 & -0.14 \\
\hline 34 & 1 & -0.12 & -0.14 & 0.04 & -0.06 & 0.02 & 0.09 & -0.05 & 0.02 & -0.16 \\
\hline 35 & 1 & -0.03 & -0.13 & 0.06 & 0.00 & 0.00 & 0.01 & -0.01 & 0.00 & 0.03 \\
\hline 36 & 6 & -0.05 & -0.33 & 0.10 & 0.00 & -0.01 & 0.01 & -0.01 & 0.00 & 0.01 \\
\hline 37 & 1 & -0.06 & -0.28 & 0.09 & 0.00 & 0.00 & 0.01 & -0.03 & 0.01 & 0.01 \\
\hline 38 & 6 & -0.02 & -0.07 & 0.04 & 0.00 & -0.01 & 0.02 & 0.00 & 0.00 & 0.00 \\
\hline 39 & 1 & -0.02 & -0.01 & 0.03 & -0.01 & -0.01 & 0.02 & 0.01 & 0.00 & 0.00 \\
\hline 40 & 6 & -0.10 & 0.13 & -0.01 & 0.00 & -0.01 & 0.02 & -0.01 & 0.00 & -0.02 \\
\hline 41 & 1 & -0.08 & -0.02 & -0.02 & 0.00 & -0.01 & 0.03 & -0.02 & -0.01 & -0.03 \\
\hline 42 & 1 & -0.05 & 0.03 & 0.01 & 0.00 & -0.03 & 0.03 & 0.00 & -0.01 & -0.03 \\
\hline 43 & 50 & -0.02 & -0.07 & 0.02 & -0.02 & -0.03 & 0.01 & -0.02 & -0.01 & -0.02 \\
\hline 44 & 6 & 0.03 & 0.15 & -0.03 & -0.04 & -0.04 & 0.01 & 0.00 & 0.02 & -0.02 \\
\hline 45 & 6 & -0.04 & -0.11 & 0.01 & -0.02 & -0.03 & 0.02 & -0.03 & -0.03 & -0.03 \\
\hline 46 & 6 & 0.03 & 0.19 & -0.01 & -0.01 & 0.01 & 0.00 & -0.04 & 0.00 & -0.02 \\
\hline 47 & 1 & 0.04 & 0.15 & -0.05 & -0.04 & -0.04 & 0.02 & -0.02 & 0.05 & -0.05 \\
\hline 48 & 1 & 0.03 & 0.16 & -0.02 & -0.04 & -0.04 & 0.01 & 0.05 & 0.04 & -0.01 \\
\hline 49 & 1 & 0.03 & 0.15 & -0.05 & -0.04 & -0.04 & 0.01 & 0.00 & -0.01 & -0.01 \\
\hline 50 & 1 & -0.03 & -0.10 & 0.00 & -0.02 & -0.02 & 0.01 & -0.02 & -0.02 & -0.05 \\
\hline 51 & 1 & -0.04 & -0.11 & 0.01 & -0.02 & -0.04 & 0.01 & -0.04 & -0.04 & -0.04 \\
\hline 52 & 1 & -0.04 & -0.11 & 0.02 & -0.02 & -0.03 & 0.03 & -0.04 & -0.03 & -0.02 \\
\hline בנה & 1 & 0.09 & 0.20 & -0.05 & -0.01 & 0.01 & -0.02 & -0.06 & 0.00 & -0.02 \\
\hline 54 & 1 & 0.03 & 0.25 & 0.03 & -0.01 & 0.02 & 0.01 & -0.03 & -0.02 & -0.02 \\
\hline 55 & 1 & 0.02 & 0.15 & -0.01 & -0.01 & 0.01 & 0.00 & -0.03 & 0.01 & -0.01 \\
\hline
\end{tabular}

thermodynamics:

Sum of electronic and zero-point Energies=

$-2653.181382$

Sum of electronic and thermal Enthalpies=

$-2653.144874$

Sum of electronic and thermal Free Energies=

$-2653.252131$

Total free energy in solution:

with all non electrostatic terms

$\left(\mathrm{a} \cdot \mathrm{u}_{\text {. })}=-2653.598961\right.$

Optimized geometry, SCF energy, three lower frequencies, thermochemistry and PCM energy for $\mathbf{A 3}$.



$\begin{array}{llc}\text { Center } & \text { Atomic } & \text { Atomic } \\ \text { Number } & \text { Number } & \text { Type }\end{array}$

$\mathrm{X}_{\mathrm{Y}}^{\text {Coordinates (Angstroms) }}$ 


\begin{tabular}{|c|c|c|c|c|c|}
\hline 1 & 6 & 0 & -4.538699 & -0.787956 & 1.933989 \\
\hline 2 & 6 & 0 & -3.710126 & -1.132841 & 0.865329 \\
\hline 3 & 6 & 0 & -3.408430 & 1.609551 & 0.628125 \\
\hline 4 & 6 & 0 & -4.273162 & 1.638752 & 1.722672 \\
\hline 5 & 6 & 0 & -4.887769 & 0.518364 & 2.292081 \\
\hline 6 & 1 & 0 & -4.898493 & -1.584997 & 2.585204 \\
\hline 7 & 1 & 0 & -4.442071 & 2.593923 & 2.220539 \\
\hline 8 & 1 & 0 & -5.542511 & 0.664164 & 3.143787 \\
\hline 9 & 15 & 0 & -2.332301 & 2.912615 & 0.138776 \\
\hline 10 & 1 & 0 & -1.930786 & 3.639612 & 1.282606 \\
\hline 11 & 1 & 0 & -2.971961 & 3.922271 & -0.610928 \\
\hline 12 & 15 & 0 & -2.948102 & -2.699912 & 0.625092 \\
\hline 13 & 1 & 0 & -2.662972 & -3.280068 & 1.882031 \\
\hline 14 & 1 & 0 & -3.808016 & -3.669691 & 0.068372 \\
\hline 15 & 16 & 0 & -1.328947 & -2.537514 & -0.614643 \\
\hline 16 & 16 & 0 & -0.784189 & 2.192904 & -0.988042 \\
\hline 17 & 46 & 0 & -0.939265 & -0.184375 & -0.800036 \\
\hline 18 & 15 & 0 & -3.117691 & 0.101056 & -0.279768 \\
\hline 19 & 6 & 0 & -4.127702 & 0.080264 & -1.807328 \\
\hline 20 & 1 & 0 & -5.184471 & 0.220396 & -1.556314 \\
\hline 21 & 1 & 0 & -3.796767 & 0.882043 & -2.475930 \\
\hline 22 & 1 & 0 & -3.993654 & -0.876934 & -2.322257 \\
\hline 23 & 1 & 0 & 6.115051 & 0.520642 & -1.647150 \\
\hline 24 & 6 & 0 & 5.023195 & 0.562454 & -1.579431 \\
\hline 25 & 50 & 0 & 4.392947 & 0.027860 & 0.399878 \\
\hline 26 & 1 & 0 & 4.706070 & 1.579945 & -1.828331 \\
\hline 27 & 1 & 0 & 4.609876 & -0.125419 & -2.323246 \\
\hline 28 & 6 & 0 & 5.112855 & 1.387254 & 1.896628 \\
\hline 29 & 6 & 0 & 2.167251 & 0.282479 & 0.462217 \\
\hline 30 & 6 & 0 & 4.821918 & -2.021085 & 0.874735 \\
\hline 31 & 1 & 0 & 6.204877 & 1.336390 & 1.954561 \\
\hline 32 & 1 & 0 & 4.705970 & 1.134396 & 2.880458 \\
\hline 33 & 1 & 0 & 4.830746 & 2.416502 & 1.655009 \\
\hline 34 & 6 & 0 & 1.539673 & -0.650187 & -0.461665 \\
\hline 35 & 1 & 0 & 1.908474 & 0.084041 & 1.507264 \\
\hline 36 & 1 & 0 & 1.984696 & 1.332963 & 0.217967 \\
\hline 37 & 1 & 0 & 4.405136 & -2.697211 & 0.121799 \\
\hline 38 & 1 & 0 & 5.905135 & -2.176792 & 0.905317 \\
\hline 39 & 1 & 0 & 4.413471 & -2.291807 & 1.853435 \\
\hline 40 & 6 & 0 & 1.124486 & -0.389085 & -1.753216 \\
\hline 41 & 1 & 0 & 1.465282 & -1.684436 & -0.121523 \\
\hline 42 & 1 & 0 & 1.326664 & 0.578863 & -2.208662 \\
\hline 43 & 1 & 0 & 0.929715 & -1.210927 & -2.439592 \\
\hline
\end{tabular}

$\mathrm{HF}=-2421.7562288$






\begin{tabular}{|c|c|c|c|c|c|c|c|c|c|c|}
\hline 16 & 16 & -0.04 & 0.02 & -0.10 & 0.06 & -0.05 & -0.03 & -0.01 & 0.03 & 0.04 \\
\hline 17 & 46 & 0.00 & 0.02 & -0.07 & 0.00 & -0.05 & -0.03 & 0.00 & 0.03 & -0.01 \\
\hline 18 & 15 & 0.01 & -0.01 & 0.03 & 0.02 & 0.03 & 0.01 & 0.00 & 0.00 & 0.00 \\
\hline 19 & 6 & -0.07 & -0.06 & 0.09 & -0.01 & 0.12 & 0.03 & -0.01 & -0.01 & 0.01 \\
\hline 20 & 1 & -0.06 & -0.09 & 0.15 & 0.00 & 0.16 & 0.05 & -0.01 & -0.03 & 0.02 \\
\hline 21 & 1 & -0.14 & -0.06 & 0.06 & 0.01 & 0.12 & 0.05 & -0.03 & 0.00 & 0.01 \\
\hline 22 & 1 & -0.08 & -0.07 & 0.09 & -0.07 & 0.13 & 0.00 & 0.00 & 0.00 & 0.00 \\
\hline 23 & 1 & 0.07 & 0.02 & 0.15 & -0.05 & 0.05 & 0.01 & -0.02 & -0.25 & -0.06 \\
\hline 24 & 6 & 0.06 & 0.03 & 0.09 & -0.05 & 0.00 & 0.00 & -0.01 & -0.24 & -0.06 \\
\hline 25 & 50 & -0.05 & -0.02 & 0.04 & -0.04 & 0.05 & 0.01 & 0.00 & -0.02 & 0.01 \\
\hline 26 & 1 & 0.09 & 0.04 & 0.11 & -0.10 & -0.02 & -0.04 & -0.02 & -0.27 & -0.16 \\
\hline 27 & 1 & 0.09 & 0.06 & 0.05 & -0.01 & -0.05 & 0.02 & -0.02 & -0.32 & 0.02 \\
\hline 28 & 6 & -0.10 & -0.08 & 0.12 & -0.13 & 0.15 & -0.04 & 0.03 & 0.14 & -0.16 \\
\hline 29 & 6 & -0.05 & 0.01 & -0.06 & -0.05 & -0.05 & -0.02 & 0.00 & 0.00 & 0.00 \\
\hline 30 & 6 & -0.11 & -0.04 & 0.00 & 0.07 & 0.09 & 0.11 & -0.01 & 0.03 & 0.24 \\
\hline 31 & 1 & -0.11 & -0.10 & 0.18 & -0.13 & 0.24 & -0.04 & 0.02 & 0.13 & -0.15 \\
\hline 32 & 1 & -0.16 & -0.11 & 0.10 & -0.11 & 0.16 & -0.03 & 0.02 & 0.27 & -0.13 \\
\hline 33 & 1 & -0.08 & -0.07 & 0.15 & -0.21 & 0.12 & -0.07 & 0.04 & 0.12 & -0.28 \\
\hline 34 & 6 & -0.02 & 0.03 & -0.10 & -0.02 & -0.08 & -0.01 & 0.00 & 0.03 & -0.02 \\
\hline 35 & 1 & -0.09 & -0.01 & -0.07 & -0.06 & -0.05 & -0.02 & 0.00 & -0.02 & 0.00 \\
\hline 36 & 1 & -0.03 & 0.01 & -0.05 & -0.08 & -0.06 & -0.03 & 0.01 & 0.01 & 0.03 \\
\hline 37 & 1 & -0.10 & -0.02 & -0.02 & 0.16 & 0.04 & 0.11 & -0.02 & -0.06 & 0.31 \\
\hline 38 & 1 & -0.11 & -0.07 & 0.03 & 0.08 & 0.17 & 0.17 & -0.01 & 0.02 & 0.25 \\
\hline 39 & 1 & -0.14 & -0.06 & -0.01 & 0.04 & 0.10 & 0.09 & -0.01 & 0.14 & 0.27 \\
\hline 40 & 6 & -0.01 & 0.06 & -0.10 & 0.00 & -0.11 & -0.02 & 0.00 & 0.06 & -0.01 \\
\hline 41 & 1 & -0.02 & 0.02 & -0.13 & -0.02 & -0.07 & 0.01 & -0.01 & 0.02 & -0.04 \\
\hline 42 & 1 & -0.01 & 0.07 & -0.07 & 0.00 & -0.12 & -0.04 & 0.00 & 0.07 & 0.01 \\
\hline 43 & 1 & 0.00 & 0.08 & -0.12 & 0.01 & -0.12 & -0.01 & 0.00 & 0.08 & -0.03 \\
\hline
\end{tabular}

thermodynamics:

Sum of electronic and zero-point Energies=

$-2421.427691$

Sum of electronic and thermal Enthalpies=

$-2421.397724$

Sum of electronic and thermal Free Energies=

$-2421.491647$

Total free energy in solution:

with all non electrostatic terms

$(\mathrm{a} \cdot \mathrm{u})=$.

\section{$\mathbf{S} \sim \mathbf{C} \sim \mathbf{S}$ complex}

Optimized geometry, SCF energy, three lower frequencies, thermochemistry and PCM energy for A1.

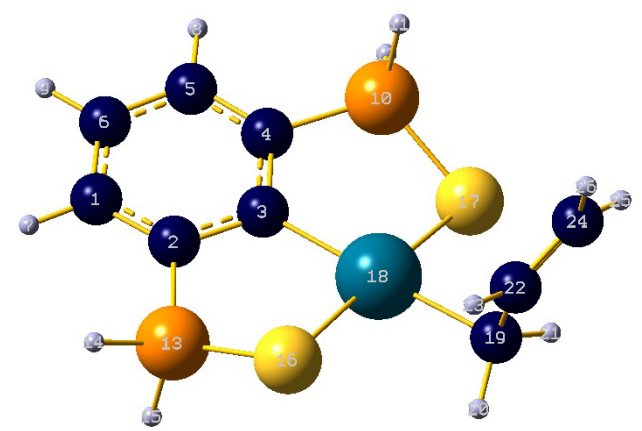

\begin{tabular}{|c|c|c|c|c|c|}
\hline \multirow{2}{*}{$\begin{array}{l}\text { Center } \\
\text { Number }\end{array}$} & \multirow{2}{*}{$\begin{array}{l}\text { Atomic } \\
\text { Number }\end{array}$} & \multirow{2}{*}{$\begin{array}{l}\text { Atomic } \\
\text { Type }\end{array}$} & \multicolumn{3}{|c|}{ Coordinates (Angstroms) } \\
\hline & & & $\mathrm{x}$ & Y & Z \\
\hline 1 & 6 & 0 & -3.546650 & -0.734063 & 0.299998 \\
\hline 2 & 6 & 0 & -2.176473 & -0.920740 & 0.088459 \\
\hline 3 & 6 & 0 & -1.239942 & 0.130674 & 0.039882 \\
\hline 4 & 6 & 0 & -1.799323 & 1.410676 & 0.226745 \\
\hline 5 & 6 & 0 & -3.159795 & 1.641962 & 0.450541 \\
\hline
\end{tabular}




\begin{tabular}{|c|c|c|c|c|c|}
\hline 6 & 6 & 0 & -4.038321 & 0.558136 & 0.485298 \\
\hline 7 & 1 & 0 & -4.236486 & -1.576249 & 0.322683 \\
\hline 8 & 1 & 0 & -3.547119 & 2.648904 & 0.597206 \\
\hline 9 & 1 & 0 & -5.099076 & 0.719952 & 0.653827 \\
\hline 10 & 15 & 0 & -0.546807 & 2.704290 & 0.182913 \\
\hline 11 & 1 & 0 & -0.262643 & 3.068610 & 1.519861 \\
\hline 12 & 1 & 0 & -1.148674 & 3.896229 & -0.284815 \\
\hline 13 & 15 & 0 & -1.420273 & -2.526903 & -0.212588 \\
\hline 14 & 1 & 0 & -2.133286 & -3.507119 & 0.516777 \\
\hline 15 & 1 & 0 & -1.750141 & -2.908546 & -1.534384 \\
\hline 16 & 16 & 0 & 0.550771 & -2.515413 & 0.160180 \\
\hline 17 & 16 & 0 & 1.067401 & 2.138443 & -0.865863 \\
\hline 18 & 46 & 0 & 0.809036 & -0.168505 & -0.280984 \\
\hline 19 & 6 & 0 & 2.881170 & -0.448699 & -0.538360 \\
\hline 20 & 1 & 0 & 2.994430 & -1.378348 & -1.105337 \\
\hline 21 & 1 & 0 & 3.290974 & 0.387601 & -1.112944 \\
\hline 22 & 6 & 0 & 3.430059 & -0.525440 & 0.823277 \\
\hline 23 & 1 & 0 & 3.294010 & -1.477268 & 1.342533 \\
\hline 24 & 6 & 0 & 4.046424 & 0.466480 & 1.491107 \\
\hline 25 & 1 & 0 & 4.211195 & 1.441113 & 1.035136 \\
\hline 26 & 1 & 0 & 4.40368 & 33. & 2.5091 \\
\hline
\end{tabular}

$\mathrm{HF}=-1955.7081585$



thermodynamics:

Sum of electronic and zero-point Energies=

$-1955.524857$

Sum of electronic and thermal Enthalpies=

$-1955.507477$

Sum of electronic and thermal Free Energies=

$-1955.570881$

Total free energy in solution:

with all non electrostatic terms

$(\mathrm{a} \cdot \mathrm{u} \cdot)=\quad-1955.717128$ 


\begin{tabular}{|c|c|c|c|c|c|}
\hline \multirow{2}{*}{$\begin{array}{l}\text { Center } \\
\text { Number }\end{array}$} & \multirow{2}{*}{$\begin{array}{l}\text { Atomic } \\
\text { Number }\end{array}$} & \multirow{2}{*}{$\begin{array}{l}\text { Atomic } \\
\text { Type }\end{array}$} & \multicolumn{3}{|c|}{ Coordinates (Angstroms) } \\
\hline & & & $\mathrm{X}$ & $\mathrm{Y}$ & Z \\
\hline 1 & 6 & 0 & -0.054094 & -0.642031 & 0.580931 \\
\hline 2 & 6 & 0 & 0.203622 & 0.246446 & 1.625344 \\
\hline 3 & 6 & 0 & 1.500767 & 0.683394 & 1.956350 \\
\hline 4 & 6 & 0 & 2.534206 & 0.168426 & 1.152922 \\
\hline 5 & 6 & 0 & 2.308893 & -0.712078 & 0.087760 \\
\hline 6 & 6 & 0 & 1.006369 & -1.117442 & -0.195346 \\
\hline 7 & 1 & 0 & -1.068227 & -0.970083 & 0.361618 \\
\hline 8 & 1 & 0 & 3.132499 & -1.086068 & -0.517153 \\
\hline 9 & 1 & 0 & 0.816002 & -1.804161 & -1.015028 \\
\hline 10 & 15 & 0 & 4.129668 & 0.773605 & 1.692927 \\
\hline 11 & 1 & 0 & 4.476728 & 1.889751 & 0.898006 \\
\hline 12 & 1 & 0 & 5.125965 & -0.151614 & 1.303462 \\
\hline 13 & 15 & 0 & -1.013623 & 0.984222 & 2.727423 \\
\hline 14 & 1 & 0 & -2.264420 & 0.956812 & 2.066620 \\
\hline 15 & 1 & 0 & -1.182894 & 0.149493 & 3.846914 \\
\hline 16 & 16 & 0 & -0.390033 & 2.836146 & 3.196165 \\
\hline 17 & 16 & 0 & 4.098567 & 1.156749 & 3.664567 \\
\hline 18 & 46 & 0 & 1.800692 & 1.882921 & 3.579140 \\
\hline 19 & 6 & 0 & 2.070299 & 3.254310 & 5.297627 \\
\hline 20 & 1 & 0 & 2.847083 & 3.897964 & 4.882029 \\
\hline 21 & 1 & 0 & 1.101075 & 3.737067 & 5.413144 \\
\hline 22 & 6 & 0 & 2.462931 & 2.365408 & 6.318853 \\
\hline 23 & 1 & 0 & 3.526963 & 2.118793 & 6.368295 \\
\hline 24 & 6 & 0 & 1.615224 & 1.642813 & 7.172951 \\
\hline 25 & 1 & 0 & 0.611588 & 2.037819 & 7.325592 \\
\hline 26 & 1 & 0 & 2.074277 & 1.224653 & 8.068712 \\
\hline 27 & 6 & 0 & 1.156322 & 0.032031 & 6.248803 \\
\hline 28 & 1 & 0 & 0.659678 & -0.419917 & 7.134034 \\
\hline 29 & 1 & 0 & 2.184352 & -0.364275 & 6.124710 \\
\hline 30 & 8 & 0 & 0.453511 & 0.271675 & 5.219194 \\
\hline
\end{tabular}

$\mathrm{HF}=-2070.1504982$

\begin{tabular}{|c|c|c|c|c|}
\hline \multicolumn{5}{|c|}{1} \\
\hline \multicolumn{5}{|c|}{${ }^{1} \mathrm{~A}$} \\
\hline Frequencies & $5--$ & -320.53 & & \\
\hline Red. masses & $5--$ & 9.89 & & \\
\hline Frc consts & -- & 0.59 & & \\
\hline IR Inten & -- & 165.30 & & \\
\hline Atom AN & $\mathrm{X}$ & Y & Z & $\mathrm{X}$ \\
\hline 16 & -0.01 & -0.02 & -0.02 & -0.01 \\
\hline 2 & 0.00 & -0.03 & -0.02 & 0.00 \\
\hline 3 & -0.01 & -0.05 & 0.00 & 0.00 \\
\hline 6 & -0.01 & -0.03 & -0.01 & -0.01 \\
\hline 6 & 0.00 & -0.01 & -0.02 & -0.02 \\
\hline 6 & 0.00 & -0.01 & -0.02 & -0.02 \\
\hline 1 & -0.01 & -0.02 & -0.04 & -0.01 \\
\hline
\end{tabular}

\section{2}

39.6043

6.2474

0.0058

0.4990 $\mathrm{Y}$

$-0.09$

0.01

0.03

$-0.04$

$-0.14$

$-0.17$

$-0.11$
-0.642031
0.246446
0.683394
0.168426
-0.712078
-1.117442
-0.970083
-1.086068
-1.804161
0.773605
1.889751
-0.151614
0.984222
0.956812
0.149493
2.836146
1.156749
1.882921
3.254310
3.897964
3.737067
2.365408
2.118793
1.642813
2.037819
1.224653
0.032031
-0.419917
-0.364275
0.271675

9194

\section{S38}




\begin{tabular}{|c|c|c|c|c|c|c|c|c|c|c|}
\hline 8 & 1 & -0.01 & 0.00 & -0.04 & -0.02 & -0.20 & 0.16 & 0.03 & -0.06 & 0.08 \\
\hline 9 & 1 & 0.00 & -0.01 & -0.03 & -0.03 & -0.25 & 0.26 & 0.03 & -0.02 & 0.02 \\
\hline 10 & 15 & -0.01 & -0.01 & 0.01 & 0.00 & -0.01 & -0.03 & 0.00 & -0.06 & 0.09 \\
\hline 11 & 1 & -0.02 & -0.01 & 0.01 & -0.01 & -0.06 & -0.11 & 0.06 & -0.09 & 0.07 \\
\hline 12 & 1 & 0.02 & 0.02 & 0.01 & -0.01 & -0.03 & 0.02 & -0.02 & -0.10 & 0.14 \\
\hline 13 & 15 & -0.02 & 0.02 & -0.06 & -0.02 & 0.07 & 0.01 & -0.01 & 0.04 & -0.05 \\
\hline 14 & 1 & 0.00 & 0.07 & -0.10 & 0.02 & 0.12 & -0.06 & -0.02 & 0.00 & -0.03 \\
\hline 15 & 1 & -0.25 & -0.04 & -0.16 & -0.12 & 0.08 & 0.00 & 0.01 & 0.06 & -0 \\
\hline 16 & 16 & 0.00 & -0.02 & 0.03 & 0.03 & 0.05 & 0.02 & -0.03 & 0.06 & -0.08 \\
\hline 17 & 16 & 0.00 & 0.01 & 0.01 & 0.04 & 0.13 & -0.05 & -0.06 & -0.01 & 0.07 \\
\hline 18 & 46 & 0.01 & 0.05 & -0.01 & 0.03 & 0.07 & -0.01 & -0.05 & 0.01 & 0.00 \\
\hline 19 & 6 & -0.01 & -0.14 & -0.17 & -0.01 & -0.04 & 0.07 & -0.10 & 0.00 & 0.02 \\
\hline 20 & 1 & 0.03 & -0.03 & 0.06 & 0.01 & -0.01 & 0.16 & -0.20 & 0.10 & -0.02 \\
\hline 21 & 1 & 0.10 & 0.03 & 0.03 & -0.01 & -0.04 & 0.07 & -0.15 & -0.12 & 0.10 \\
\hline 22 & 6 & 0.04 & 0.04 & 0.14 & -0.06 & -0.13 & 0.00 & 0.08 & 0.02 & -0.02 \\
\hline 23 & 1 & 0.04 & 0.03 & 0.13 & -0.07 & -0.15 & 0.02 & 0.11 & 0.15 & -0.11 \\
\hline 24 & 6 & 0.19 & 0.48 & 0.36 & -0.09 & -0.20 & -0.08 & 0.23 & -0.10 & 0.01 \\
\hline 25 & 1 & 0.00 & 0.05 & 0.11 & -0.10 & -0.21 & -0.09 & 0.20 & -0.21 & 0.12 \\
\hline 26 & 1 & 0.08 & 0.00 & 0.17 & -0.12 & -0.25 & -0.09 & 0.34 & -0.13 & -0.06 \\
\hline 27 & 6 & -0.12 & -0.42 & -0.22 & -0.04 & -0.16 & -0.16 & 0.25 & -0.06 & -0.12 \\
\hline 28 & 1 & 0.00 & 0.10 & 0.09 & -0.03 & -0.22 & -0.18 & 0.37 & -0.18 & -0.11 \\
\hline 29 & 1 & 0.03 & 0.02 & -0.15 & -0.04 & -0.13 & -0.18 & 0.27 & 0.03 & -0.2 \\
\hline 30 & 8 & -0.08 & -0.17 & 0.05 & -0.06 & -0.13 & -0.15 & 0.14 & 0.01 & -0.0 \\
\hline
\end{tabular}

thermodynamics:

Sum of electronic and zero-point Energies=

Sum of electronic and thermal Enthalpies=

Sum of electronic and thermal Free Energies=
$-2069.936100$

$-2069.916866$

$-2069.983101$

Total free energy in solution:

with all non electrostatic terms

$($ a.u. $)=-2070.158662$

Optimized geometry, SCF energy, three lower frequencies, thermochemistry and PCM energy for $\mathbf{A 2}$.



\begin{tabular}{|c|c|c|c|c|c|}
\hline \multirow{2}{*}{$\begin{array}{l}\text { Center } \\
\text { Number }\end{array}$} & \multirow{2}{*}{$\begin{array}{l}\text { Atomic } \\
\text { Number }\end{array}$} & \multirow{2}{*}{$\begin{array}{l}\text { Atomic } \\
\text { Type }\end{array}$} & \multicolumn{3}{|c|}{ Coordinates (Angstroms) } \\
\hline & & & $\mathrm{x}$ & Y & Z \\
\hline 1 & 6 & 0 & -3.693235 & 1.657164 & 0.528864 \\
\hline 2 & 6 & 0 & -2.340377 & 1.429654 & 0.258596 \\
\hline 3 & 6 & 0 & -1.784979 & 0.143073 & 0.063738 \\
\hline 4 & 6 & 0 & -2.721909 & -0.911268 & 0.171808 \\
\hline 5 & 6 & 0 & -4.080441 & -0.715855 & 0.439470 \\
\hline 6 & 6 & 0 & -4.569848 & 0.577079 & 0.617326 \\
\hline 7 & 1 & 0 & -4.073906 & 2.667026 & 0.671701 \\
\hline 8 & 1 & 0 & -4.763828 & -1.560048 & 0.513503 \\
\hline 9 & 1 & 0 & -5.622883 & 0.741066 & 0.825712 \\
\hline 10 & 15 & 0 & -2.005203 & -2.540234 & -0.086114 \\
\hline 11 & 1 & 0 & -2.463021 & -3.014929 & -1.338031 \\
\hline
\end{tabular}




$\begin{array}{rr}12 & 1 \\ 13 & 15 \\ 14 & 1 \\ 15 & 1 \\ 16 & 16 \\ 17 & 16 \\ 18 & 46 \\ 19 & 6 \\ 20 & 1 \\ 21 & 1 \\ 22 & 6 \\ 23 & 1 \\ 24 & 6 \\ 25 & 1 \\ 26 & 1 \\ 27 & 8 \\ 28 & 6 \\ 29 & 1 \\ 30 & 1\end{array}$

$\begin{array}{rrr}-2.665828 & -3.440738 & 0.781524 \\ -1.125275 & 2.752748 & 0.172943 \\ -1.748971 & 3.881044 & -0.407481 \\ -0.922989 & 3.224254 & 1.491716 \\ 0.541045 & 2.148672 & -0.762745 \\ -0.009986 & -2.498184 & 0.100145 \\ 0.186381 & -0.158213 & -0.299045 \\ 4.444665 & -0.424481 & -0.244343 \\ 4.496944 & -1.420914 & -0.705994 \\ 4.603336 & 0.309896 & -1.043170 \\ 5.492929 & -0.288728 & 0.816498 \\ 5.452194 & -1.023358 & 1.624238 \\ 6.437771 & 0.652649 & 0.855466 \\ 6.519616 & 1.408306 & 0.075691 \\ 7.167252 & 0.702212 & 1.660733 \\ 2.113078 & -0.473139 & -0.730421 \\ 3.014165 & -0.253459 & 0.303566 \\ 2.933307 & 0.762270 & 0.736881 \\ 2.878431 & -0.963673 & 1.145661\end{array}$

$\mathrm{HF}=-2070.19125$

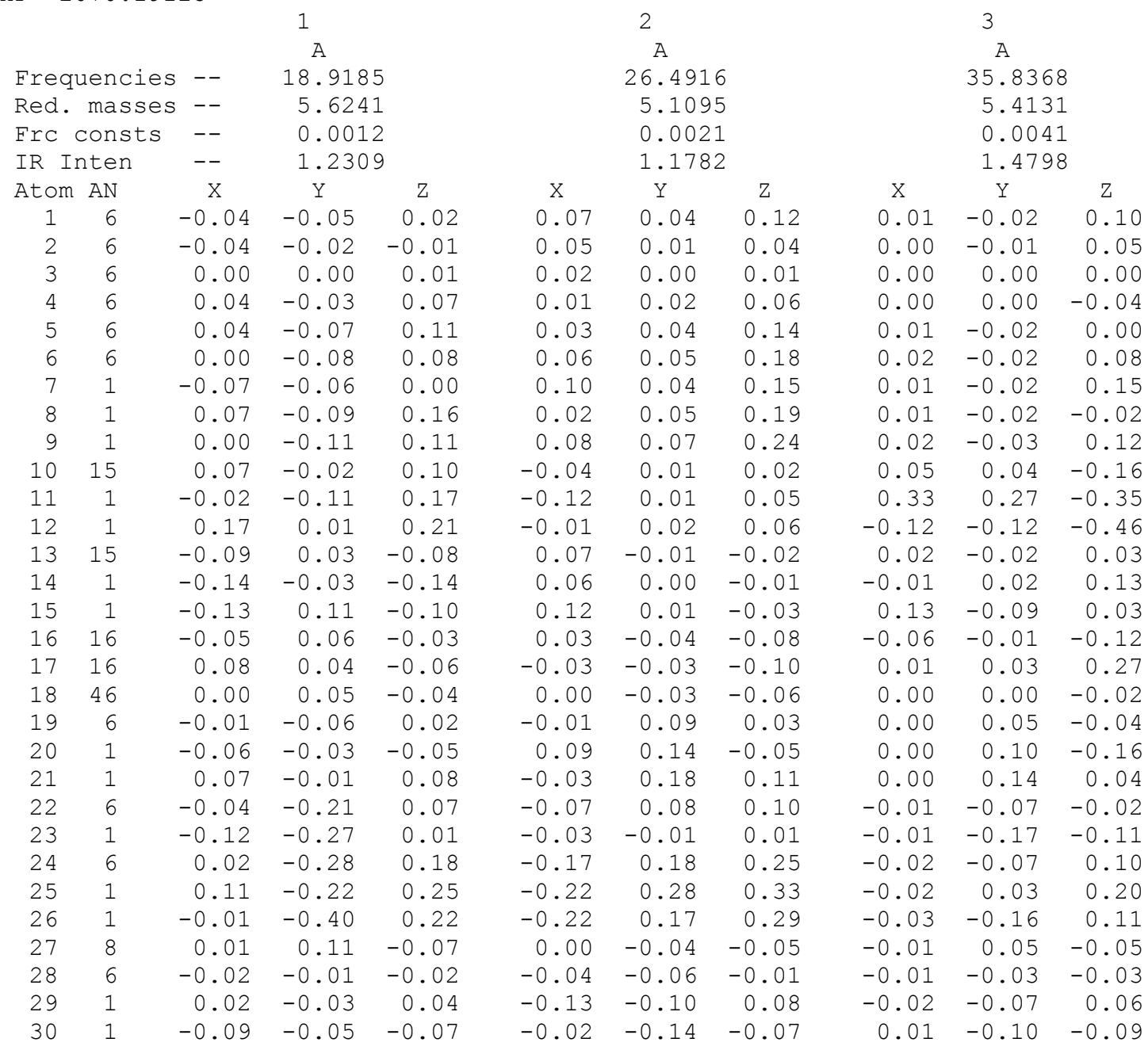

thermodynamics:

Sum of electronic and zero-point Energies=

$-2069.974574$

Sum of electronic and thermal Enthalpies=

$-2069.954613$

Sum of electronic and thermal Free Energies=

$-2070.025666$

Total free energy in solution: 


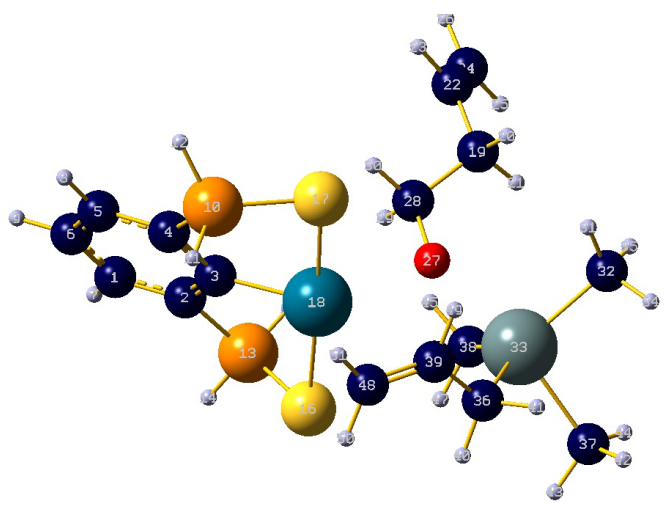

\begin{tabular}{|c|c|c|c|c|c|}
\hline Center & Atomic & Atomic & Coor & inates (An & troms) \\
\hline Number & Number & Type & $\mathrm{X}$ & $\mathrm{Y}$ & Z \\
\hline 1 & 6 & 0 & 4.126820 & 0.550267 & 2.357486 \\
\hline 2 & 6 & 0 & 2.921780 & 0.122272 & 1.796274 \\
\hline 3 & 6 & 0 & 2.751236 & -0.094177 & 0.408189 \\
\hline 4 & 6 & 0 & 3.898972 & 0.157138 & -0.378849 \\
\hline 5 & 6 & 0 & 5.117544 & 0.577137 & 0.161192 \\
\hline 6 & 6 & 0 & 5.232060 & 0.774963 & 1.536549 \\
\hline 7 & 1 & 0 & 4.212873 & 0.711412 & 3.429941 \\
\hline 8 & 1 & 0 & 5.977906 & 0.753936 & -0.480674 \\
\hline 9 & 1 & 0 & 6.173252 & 1.105257 & 1.965033 \\
\hline 10 & 15 & 0 & 3.565021 & -0.119198 & -2.111758 \\
\hline 11 & 1 & 0 & 3.935140 & -1.445128 & -2.431468 \\
\hline 12 & 1 & 0 & 4.487744 & 0.624160 & -2.881956 \\
\hline 13 & 15 & 0 & 1.413772 & -0.233371 & 2.692855 \\
\hline 14 & 1 & 0 & 1.738828 & -0.558902 & 4.029068 \\
\hline 15 & 1 & 0 & 0.676376 & 0.959290 & 2.843751 \\
\hline 16 & 16 & 0 & 0.449895 & -1.681838 & 1.695793 \\
\hline 17 & 16 & 0 & 1.637823 & 0.313110 & -2.468181 \\
\hline 18 & 46 & 0 & 0.991512 & -0.624488 & -0.370028 \\
\hline 19 & 6 & 0 & -1.694255 & 2.971019 & -0.723292 \\
\hline 20 & 1 & 0 & -1.943255 & 2.816770 & -1.782245 \\
\hline 21 & 1 & 0 & -2.618247 & 2.832318 & -0.150552 \\
\hline 22 & 6 & 0 & -1.161581 & 4.356263 & -0.519467 \\
\hline 23 & 1 & 0 & -0.300543 & 4.630148 & -1.134393 \\
\hline 24 & 6 & 0 & -1.630143 & 5.248458 & 0.355491 \\
\hline 25 & 1 & 0 & -2.485466 & 5.026508 & 0.991741 \\
\hline 26 & 1 & 0 & -1.186339 & 6.235681 & 0.463420 \\
\hline 27 & 8 & 0 & -1.034967 & 0.589031 & -0.619292 \\
\hline 28 & 6 & 0 & -0.658352 & 1.899140 & -0.326312 \\
\hline 29 & 1 & 0 & -0.438254 & 2.046382 & 0.752360 \\
\hline 30 & 1 & 0 & 0.280164 & 2.154649 & -0.851482 \\
\hline 31 & 1 & 0 & -3.527493 & 1.092125 & -2.144388 \\
\hline 32 & 6 & 0 & -4.157393 & 0.740250 & -1.323798 \\
\hline 33 & 50 & 0 & -3.017740 & -0.366967 & 0.148590 \\
\hline 34 & 1 & 0 & -4.930648 & 0.075813 & -1.726396 \\
\hline 35 & 1 & 0 & -4.662343 & 1.604930 & -0.878658 \\
\hline 36 & 6 & 0 & -2.163141 & -2.181640 & -0.821070 \\
\hline 37 & 6 & 0 & -4.683037 & -1.588316 & 0.970827 \\
\hline 38 & 6 & 0 & -2.565091 & 0.519538 & 2.086155 \\
\hline 39 & 6 & 0 & -0.967943 & -2.076202 & -1.663488 \\
\hline 40 & 1 & 0 & -2.069874 & -2.966145 & -0.060953 \\
\hline 41 & 1 & 0 & -3.029290 & -2.432121 & -1.449530 \\
\hline 42 & 1 & 0 & -5.167728 & -2.197150 & 0.196129 \\
\hline 43 & 1 & 0 & -4.337106 & -2.269950 & 1.759448 \\
\hline 44 & 1 & 0 & -5.453391 & -0.942605 & 1.412918 \\
\hline 45 & 1 & 0 & -1.766702 & 1.266139 & 2.082892 \\
\hline
\end{tabular}




$\begin{array}{rrrrrr}46 & 1 & 0 & -3.479960 & 0.986595 & 2.470114 \\ 47 & 1 & 0 & -2.298633 & -0.292398 & 2.772401 \\ 48 & 6 & 0 & 0.202281 & -2.752323 & -1.501818 \\ 49 & 1 & 0 & -1.045958 & -1.410778 & -2.524014 \\ 50 & 1 & 0 & 0.320155 & -3.503390 & -0.722832 \\ 51 & 1 & 0 & 0.992048 & -2.695518 & -2.246239\end{array}$

\begin{tabular}{|c|c|c|c|c|c|c|c|c|c|c|}
\hline \multicolumn{3}{|c|}{ (n) } & \multicolumn{2}{|l|}{1} & \multicolumn{3}{|c|}{2} & \multicolumn{3}{|c|}{3} \\
\hline & & \multicolumn{3}{|c|}{ A } & \multicolumn{3}{|c|}{ A } & \\
\hline \multicolumn{2}{|c|}{ Frequencies } & -- & \multicolumn{2}{|c|}{-100.6193} & \multicolumn{3}{|c|}{10.3674} & \multicolumn{3}{|c|}{$\begin{array}{c}A \\
28.4818\end{array}$} \\
\hline \multicolumn{2}{|c|}{ Red. masses } & -- & \multicolumn{2}{|c|}{15.1579} & \multicolumn{3}{|c|}{5.2663} & \multicolumn{3}{|c|}{4.4924} \\
\hline Frc & consts & -- & 0.090 & & & 0.000 & & & 0.002 & \\
\hline IR Ir & nten & -- & 10.484 & & & 0.136 & & & 0.209 & \\
\hline Atom & AN & $\mathrm{X}$ & $\mathrm{Y}$ & Z & $\mathrm{X}$ & $\mathrm{Y}$ & Z & $\mathrm{X}$ & $\mathrm{Y}$ & Z \\
\hline 1 & 6 & 0.08 & -0.09 & -0.04 & 0.03 & 0.10 & -0.05 & -0.06 & 0.01 & 0.0 \\
\hline 2 & 6 & 0.10 & -0.18 & -0.02 & 0.02 & 0.07 & -0.03 & -0.06 & 0.02 & 0.0 \\
\hline 3 & 6 & 0.10 & -0.24 & -0.01 & 0.01 & 0.02 & -0.02 & -0.02 & 0.00 & 0.0 \\
\hline 4 & 6 & 0.08 & -0.18 & -0.03 & 0.00 & 0.01 & -0.04 & 0.01 & -0.04 & 0.0 \\
\hline 5 & 6 & 0.06 & -0.09 & -0.05 & 0.00 & 0.04 & -0.07 & 0.00 & -0.05 & 0.0 \\
\hline 6 & 6 & 0.06 & -0.05 & -0.06 & 0.01 & 0.09 & -0.07 & -0.04 & -0.03 & 0.0 \\
\hline 7 & 1 & 0.07 & -0.03 & -0.05 & 0.04 & 0.14 & -0.06 & -0.09 & 0.02 & 0.0 \\
\hline 8 & 1 & 0.03 & -0.03 & -0.07 & -0.01 & 0.03 & -0.08 & 0.02 & -0.08 & 0.0 \\
\hline 9 & 1 & 0.04 & 0.02 & -0.07 & 0.01 & 0.11 & -0.10 & -0.04 & -0.03 & 0.0 \\
\hline 10 & 15 & 0.01 & -0.07 & -0.03 & -0.02 & -0.04 & -0.03 & 0.05 & -0.07 & 0.0 \\
\hline 11 & 1 & -0.12 & -0.09 & -0.11 & -0.01 & -0.05 & 0.01 & 0.03 & -0.09 & 0.0 \\
\hline 12 & 1 & 0.05 & -0.11 & -0.02 & -0.04 & -0.06 & -0.06 & 0.09 & -0.11 & 0.0 \\
\hline 13 & 15 & 0.08 & -0.04 & 0.02 & 0.04 & 0.08 & 0.01 & -0.08 & 0.05 & -0.0 \\
\hline 14 & 1 & 0.10 & -0.11 & 0.00 & 0.06 & 0.11 & 0.01 & -0.12 & 0.05 & -0.0 \\
\hline 15 & 1 & 0.25 & 0.05 & 0.09 & 0.04 & 0.08 & -0.01 & -0.07 & 0.06 & -0.0 \\
\hline 16 & 16 & -0.13 & 0.13 & 0.02 & 0.03 & 0.05 & 0.06 & -0.07 & 0.05 & -0.0 \\
\hline 17 & 16 & 0.03 & 0.10 & 0.04 & -0.03 & -0.07 & -0.02 & 0.07 & -0.03 & -0.0 \\
\hline 18 & 46 & -0.06 & 0.25 & 0.08 & 0.01 & -0.02 & 0.02 & -0.01 & 0.01 & -0.0 \\
\hline 19 & 6 & 0.03 & -0.07 & 0.00 & 0.01 & -0.01 & 0.16 & -0.09 & -0.01 & 0.0 \\
\hline 20 & 1 & 0.01 & -0.06 & 0.00 & -0.05 & 0.05 & 0.17 & -0.24 & 0.03 & 0.1 \\
\hline 21 & 1 & 0.06 & -0.11 & 0.03 & 0.04 & -0.03 & 0.21 & -0.01 & -0.07 & 0.2 \\
\hline 22 & 6 & 0.00 & -0.06 & 0.00 & 0.03 & -0.02 & 0.21 & -0.12 & 0.00 & 0.0 \\
\hline 23 & 1 & -0.01 & -0.04 & -0.02 & 0.00 & 0.00 & 0.18 & -0.21 & 0.06 & -0.0 \\
\hline 24 & 6 & -0.01 & -0.07 & 0.00 & 0.08 & -0.06 & 0.27 & -0.04 & -0.05 & 0.1 \\
\hline 25 & 1 & 0.00 & -0.09 & 0.01 & 0.11 & -0.09 & 0.31 & 0.04 & -0.11 & 0.2 \\
\hline 26 & 1 & -0.04 & -0.05 & -0.02 & 0.10 & -0.07 & 0.30 & -0.07 & -0.04 & 0.1 \\
\hline 27 & 8 & 0.13 & -0.05 & -0.09 & 0.00 & -0.02 & 0.00 & -0.01 & 0.01 & -0.0 \\
\hline 28 & 6 & 0.09 & -0.04 & -0.05 & 0.03 & -0.04 & 0.04 & 0.00 & 0.01 & -0.1 \\
\hline 29 & 1 & 0.10 & -0.07 & -0.05 & 0.10 & -0.09 & 0.04 & 0.17 & -0.01 & -0.1 \\
\hline 30 & 1 & 0.06 & 0.03 & -0.06 & 0.00 & -0.01 & 0.00 & -0.09 & 0.05 & -0.2 \\
\hline 31 & 1 & 0.09 & -0.04 & -0.03 & 0.12 & -0.05 & -0.11 & -0.04 & -0.14 & -0.0 \\
\hline 32 & 6 & 0.07 & -0.03 & -0.04 & 0.07 & 0.00 & -0.13 & -0.02 & -0.07 & 0 \\
\hline 33 & 50 & -0.02 & -0.08 & -0.02 & -0.03 & 0.00 & -0.05 & 0.04 & 0.01 & 0. \\
\hline 34 & 1 & 0.06 & -0.01 & -0.06 & 0.06 & 0.02 & -0.14 & -0.05 & -0.08 & 0.0 \\
\hline 35 & 1 & 0.08 & -0.02 & -0.05 & 0.09 & 0.04 & -0.18 & 0.02 & -0.03 & -0 \\
\hline 36 & 6 & -0.01 & -0.08 & 0.06 & -0.01 & -0.03 & 0.03 & 0.03 & -0.01 & 0 \\
\hline 37 & 6 & -0.07 & 0.04 & 0.01 & -0.09 & 0.05 & -0.11 & 0.11 & 0.00 & 0. \\
\hline 38 & 6 & 0.04 & 0.00 & -0.07 & -0.07 & 0.00 & -0.04 & 0.05 & 0.13 & -0 \\
\hline 39 & 6 & -0.04 & -0.15 & -0.04 & 0.01 & -0.06 & 0.05 & 0.00 & 0.00 & $-0 . c$ \\
\hline 40 & 1 & 0.06 & -0.03 & 0.10 & -0.02 & 0.00 & 0.06 & 0.05 & 0.00 & 0 \\
\hline 41 & 1 & -0.03 & -0.20 & 0.13 & 0.01 & -0.05 & 0.02 & 0.01 & -0.01 & 0. \\
\hline 42 & 1 & -0.10 & 0.04 & 0.02 & -0.07 & 0.05 & -0.12 & 0.08 & -0.04 & 0.1 \\
\hline 43 & 1 & -0.10 & 0.03 & 0.02 & -0.14 & 0.06 & -0.08 & 0.16 & 0.02 & 0.1 \\
\hline 44 & 1 & -0.05 & 0.07 & 0.00 & -0.10 & 0.08 & -0.15 & 0.11 & -0.01 & 0.1 \\
\hline 45 & 1 & 0.13 & -0.10 & -0.09 & -0.08 & 0.01 & -0.03 & 0.08 & 0.10 & -0.0 \\
\hline 46 & 1 & 0.08 & 0.13 & -0.14 & -0.08 & -0.02 & -0.05 & 0.07 & 0.20 & $-0 . c$ \\
\hline 47 & 1 & -0.08 & 0.01 & -0.02 & -0.06 & 0.00 & -0.05 & 0.01 & 0.18 & 0.0 \\
\hline 48 & 6 & -0.09 & -0.19 & -0.11 & 0.01 & -0.05 & 0.10 & 0.01 & 0.01 & -0.0 \\
\hline 49 & 1 & -0.06 & -0.13 & -0.02 & 0.03 & -0.09 & 0.02 & -0.03 & -0.01 & -0.0 \\
\hline 50 & 1 & -0.02 & -0.13 & -0.05 & -0.01 & -0.03 & 0.13 & 0.04 & 0.01 & -0.0 \\
\hline 51 & 1 & -0.05 & -0.12 & -0.06 & 0.02 & -0.08 & 0.11 & -0.01 & 0.01 & -0 \\
\hline
\end{tabular}


Sum of electronic and zero-point Energies=

Sum of electronic and thermal Enthalpies=

Sum of electronic and thermal Free Energies=

Total free energy in solution:

with all non electrostatic terms
$-2310.104263$

$-2310.071360$

$-2310.169469$

$($ a.u. $)=-2310.497378$

Optimized geometry, SCF energy, three lower frequencies, thermochemistry and PCM energy for A3.

\begin{tabular}{|c|c|c|c|c|c|}
\hline \multirow{2}{*}{$\begin{array}{l}\text { Center } \\
\text { Number }\end{array}$} & \multirow{2}{*}{$\begin{array}{l}\text { Atomic } \\
\text { Number }\end{array}$} & \multirow{2}{*}{$\begin{array}{c}\text { Atomic } \\
\text { Type }\end{array}$} & \multicolumn{3}{|c|}{ Coordinates (Angstroms) } \\
\hline & & & $x$ & Y & Z \\
\hline 1 & 6 & 0 & .098761 & .246338 & -.170283 \\
\hline 2 & 6 & 0 & .039668 & .282842 & 1.227552 \\
\hline 3 & 6 & 0 & 1.169366 & .103456 & 2.055215 \\
\hline 4 & 6 & 0 & 2.380827 & -.105795 & 1.361362 \\
\hline 5 & 6 & 0 & 2.469150 & -.136609 & -.035407 \\
\hline 6 & 6 & 0 & 1.320936 & .036224 & -.803762 \\
\hline 7 & 1 & 0 & -.797386 & .385755 & -.770576 \\
\hline 8 & 1 & 0 & 3.423830 & -.297934 & -.530853 \\
\hline 9 & 1 & 0 & 1.378448 & .010055 & -1.887259 \\
\hline 10 & 15 & 0 & 3.803393 & -.311802 & 2.429718 \\
\hline 11 & 1 & 0 & 4.539761 & .891116 & 2.448158 \\
\hline 12 & 1 & 0 & 4.713337 & -1.207643 & 1.834028 \\
\hline 13 & 15 & 0 & -1.479052 & .568226 & 2.133070 \\
\hline 14 & 1 & 0 & -2.315282 & 1.420428 & 1.384870 \\
\hline 15 & 1 & 0 & -2.226779 & -.628065 & 2.163601 \\
\hline 16 & 16 & 0 & -1.017867 & 1.299007 & 3.953705 \\
\hline 17 & 16 & 0 & 3.171986 & -.913046 & 4.247173 \\
\hline 18 & 46 & 0 & 1.061985 & .165349 & 4.092221 \\
\hline 19 & 1 & 0 & -2.904210 & -3.079361 & 9.976346 \\
\hline 20 & 6 & 0 & -2.678316 & -2.812802 & 8.938888 \\
\hline 21 & 50 & 0 & -.849789 & -1.693629 & 8.848311 \\
\hline 22 & 1 & 0 & -2.596768 & -3.736732 & 8.358312 \\
\hline 23 & 1 & 0 & -3.513565 & -2.224681 & 8.546859 \\
\hline 24 & 6 & 0 & -.581349 & -1.278658 & 6.658398 \\
\hline 25 & 6 & 0 & .850406 & -2.831434 & 9.497057 \\
\hline 26 & 6 & 0 & -.983486 & .213732 & 9.819838 \\
\hline 27 & 6 & 0 & .732111 & -.694984 & 6.438958 \\
\hline 28 & 1 & 0 & -.694185 & -2.262790 & 6.192292 \\
\hline 29 & 1 & 0 & -1.409356 & -.622425 & 6.376536 \\
\hline 30 & 1 & 0 & 1.764549 & -2.231015 & 9.458424 \\
\hline 31 & 1 & 0 & .705628 & -3.160052 & 10.531217 \\
\hline 32 & 1 & 0 & .988493 & -3.722048 & 8.875915 \\
\hline 33 & 1 & 0 & -1.859942 & .768575 & 9.471335 \\
\hline 34 & 1 & 0 & -1.075580 & .075724 & 10.901964 \\
\hline 35 & 1 & 0 & -.089072 & .814873 & 9.629582 \\
\hline 36 & 6 & 0 & 1.042738 & .647063 & 6.311322 \\
\hline 37 & 1 & 0 & 1.570697 & -1.392735 & 6.483149 \\
\hline 38 & 1 & 0 & .272272 & 1.401291 & 6.458697 \\
\hline 39 & 1 & 0 & 2.069989 & .981695 & 6.435414 \\
\hline
\end{tabular}




\begin{tabular}{|c|c|c|c|c|c|c|c|c|c|c|}
\hline \multirow{2}{*}{\multicolumn{2}{|c|}{ Frequencies }} & \multicolumn{3}{|c|}{ A } & \multicolumn{3}{|c|}{ A } & \multicolumn{3}{|c|}{ A } \\
\hline & & -- & 15.157 & & & 21.421 & & & 27.446 & \\
\hline Red. & masses & -- & 3.733 & & & 5.242 & & & 5.999 & \\
\hline FrC & consts & -- & .000 & & & .001 & & & .002 & \\
\hline IR I & nten & -- & .143 & & & .839 & & & .294 & \\
\hline Atom & AN & X & Y & Z & $\mathrm{x}$ & Y & Z & $\mathrm{x}$ & Y & Z \\
\hline 1 & 6 & .01 & .02 & -.01 & .07 & .17 & -.05 & .10 & -.01 & -.06 \\
\hline 2 & 6 & .00 & -.01 & -.01 & .04 & .08 & -.05 & .05 & .01 & -.06 \\
\hline 3 & 6 & .01 & .01 & -.01 & .02 & .03 & -.03 & .01 & .00 & -.01 \\
\hline 4 & 6 & .02 & .06 & -.01 & .03 & .06 & -.01 & .03 & -.05 & .04 \\
\hline 5 & 6 & .02 & .10 & -.01 & .07 & .15 & -.01 & .08 & -.08 & .04 \\
\hline 6 & 6 & .02 & .07 & -.01 & .09 & .20 & -.03 & .11 & -.06 & -.01 \\
\hline 7 & 1 & .01 & .01 & -.01 & .09 & .21 & -.06 & .12 & .00 & -.09 \\
\hline 8 & 1 & .03 & .14 & -.01 & .08 & .17 & .01 & .09 & -.11 & .08 \\
\hline 9 & 1 & .03 & .10 & -.01 & .12 & .27 & -.03 & .15 & -.08 & .00 \\
\hline 10 & 15 & .02 & .09 & .00 & .00 & -.01 & .02 & -.01 & -.06 & .08 \\
\hline 11 & 1 & -.02 & .11 & .03 & .02 & -.02 & .10 & .03 & -.09 & .07 \\
\hline 12 & 1 & .05 & .13 & -.02 & .01 & .01 & -.01 & -.02 & -.11 & .14 \\
\hline 13 & 15 & -.01 & -.07 & -.01 & .02 & .04 & -.07 & .01 & .07 & -.13 \\
\hline 14 & 1 & -.03 & -.09 & .00 & .03 & .07 & -.04 & .08 & .10 & -.16 \\
\hline 15 & 1 & .03 & -.10 & -.02 & .02 & .04 & -.14 & -.03 & .09 & -.17 \\
\hline 16 & 16 & -.03 & -.08 & .00 & -.02 & -.05 & -.02 & -.05 & .04 & -.10 \\
\hline 17 & 16 & .03 & .02 & -.02 & -.05 & -.10 & -.03 & -.08 & .01 & .08 \\
\hline 18 & 46 & .00 & -.02 & -.01 & -.03 & -.06 & -.03 & -.06 & .04 & -.02 \\
\hline 19 & 1 & -.12 & .20 & .05 & .12 & -.04 & .17 & .07 & -.01 & .09 \\
\hline 20 & 6 & -.12 & .20 & .05 & .07 & -.08 & .15 & .01 & .04 & .09 \\
\hline 21 & 50 & -.01 & .01 & .01 & .01 & .02 & .05 & .05 & -.03 & .03 \\
\hline 22 & 1 & -.23 & .21 & .03 & .12 & -.11 & .20 & -.07 & .07 & .03 \\
\hline 23 & 1 & -.07 & .30 & .09 & .03 & -.15 & .14 & .01 & .09 & .18 \\
\hline 24 & 6 & .01 & -.06 & .00 & -.06 & -.06 & .02 & -.06 & .04 & .03 \\
\hline 25 & 6 & -.12 & -.15 & .02 & .09 & .16 & .06 & .04 & -.13 & -.13 \\
\hline 26 & 6 & .22 & .06 & -.04 & -.07 & .07 & -.05 & .19 & -.07 & .12 \\
\hline 27 & 6 & .00 & -.05 & -.01 & -.05 & -.09 & -.01 & -.08 & .08 & .00 \\
\hline 28 & 1 & .01 & -.07 & .02 & -.10 & -.06 & .05 & -.04 & .04 & .01 \\
\hline 29 & 1 & .00 & -.07 & -.01 & -.06 & -.04 & .03 & -.09 & .02 & .06 \\
\hline 30 & 1 & -.06 & -.25 & .01 & .06 & .21 & -.01 & .06 & -.16 & -.15 \\
\hline 31 & 1 & -.15 & -.12 & .03 & .15 & .21 & .08 & .09 & -.17 & -.13 \\
\hline 32 & 1 & -.21 & -.18 & .04 & .12 & .13 & .10 & -.03 & -.10 & -.17 \\
\hline 33 & 1 & .21 & .10 & .03 & -.16 & -.04 & .01 & .21 & .00 & .17 \\
\hline 34 & 1 & .33 & .11 & -.03 & .07 & .13 & -.03 & .21 & -.11 & .12 \\
\hline 35 & 1 & .24 & -.01 & -.17 & -.15 & .14 & -.19 & .22 & -.11 & .12 \\
\hline 36 & 6 & .00 & -.05 & .00 & -.03 & -.10 & -.02 & -.13 & .09 & -.03 \\
\hline 37 & 1 & .01 & -.05 & -.01 & -.07 & -.11 & -.03 & -.06 & .11 & .02 \\
\hline 38 & 1 & -.01 & -.06 & .00 & -.01 & -.08 & -.01 & -.15 & .06 & -.05 \\
\hline 39 & 1 & -.01 & -.05 & .00 & -.02 & -.12 & -.03 & -.14 & .13 & -.03 \\
\hline
\end{tabular}

thermodynamics:

Sum of electronic and zero-point Energies=

$-2078.345736$

Sum of electronic and thermal Enthalpies=

$-2078.318890$

Sum of electronic and thermal Free Energies=

$-2078.406269$

Total free energy in solution:

with all non electrostatic terms

$(\mathrm{a} \cdot \mathrm{u})=-2078.676795$

Optimized geometry, SCF energy, three lower frequencies, thermochemistry and PCM energy for allylalcoolate. 


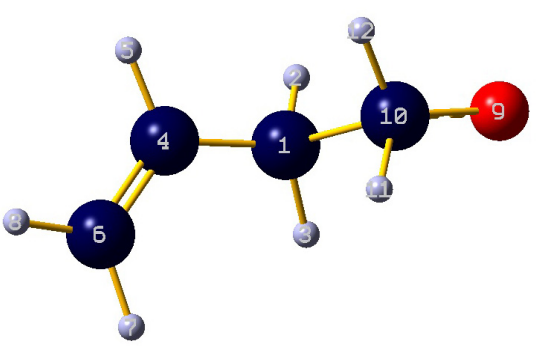

\begin{tabular}{|c|c|c|c|c|c|}
\hline \multirow{2}{*}{$\begin{array}{l}\text { Center } \\
\text { Number }\end{array}$} & \multirow{2}{*}{$\begin{array}{l}\text { Atomic } \\
\text { Number }\end{array}$} & \multirow{2}{*}{$\begin{array}{l}\text { Atomic } \\
\text { Type }\end{array}$} & \multicolumn{3}{|c|}{ Coordinates (Angstroms) } \\
\hline & & & $\mathrm{x}$ & $\mathrm{Y}$ & Z \\
\hline 1 & 6 & 0 & 0.017655 & 0.039665 & 0.110581 \\
\hline 2 & 1 & 0 & -0.087690 & -0.065856 & 1.198262 \\
\hline 3 & 1 & 0 & 1.088448 & 0.021017 & -0.131666 \\
\hline 4 & 6 & 0 & -0.641419 & 1.262869 & -0.386640 \\
\hline 5 & 1 & 0 & -1.617229 & 1.488453 & 0.057694 \\
\hline 6 & 6 & 0 & -0.248716 & 2.036426 & -1.415891 \\
\hline 7 & 1 & 0 & 0.702446 & 1.866640 & -1.920525 \\
\hline 8 & 1 & 0 & -0.861317 & 2.853832 & -1.796168 \\
\hline 9 & 8 & 0 & -0.068910 & -2.416258 & 0.036308 \\
\hline 10 & 6 & 0 & -0.595317 & -1.355864 & -0.495573 \\
\hline 11 & 1 & 0 & -0.447832 & -1.189394 & -1.618086 \\
\hline 12 & 1 & 0 & -1.728512 & -1.208330 & -0.371826 \\
\hline
\end{tabular}

$\mathrm{HF}=-231.7069282$

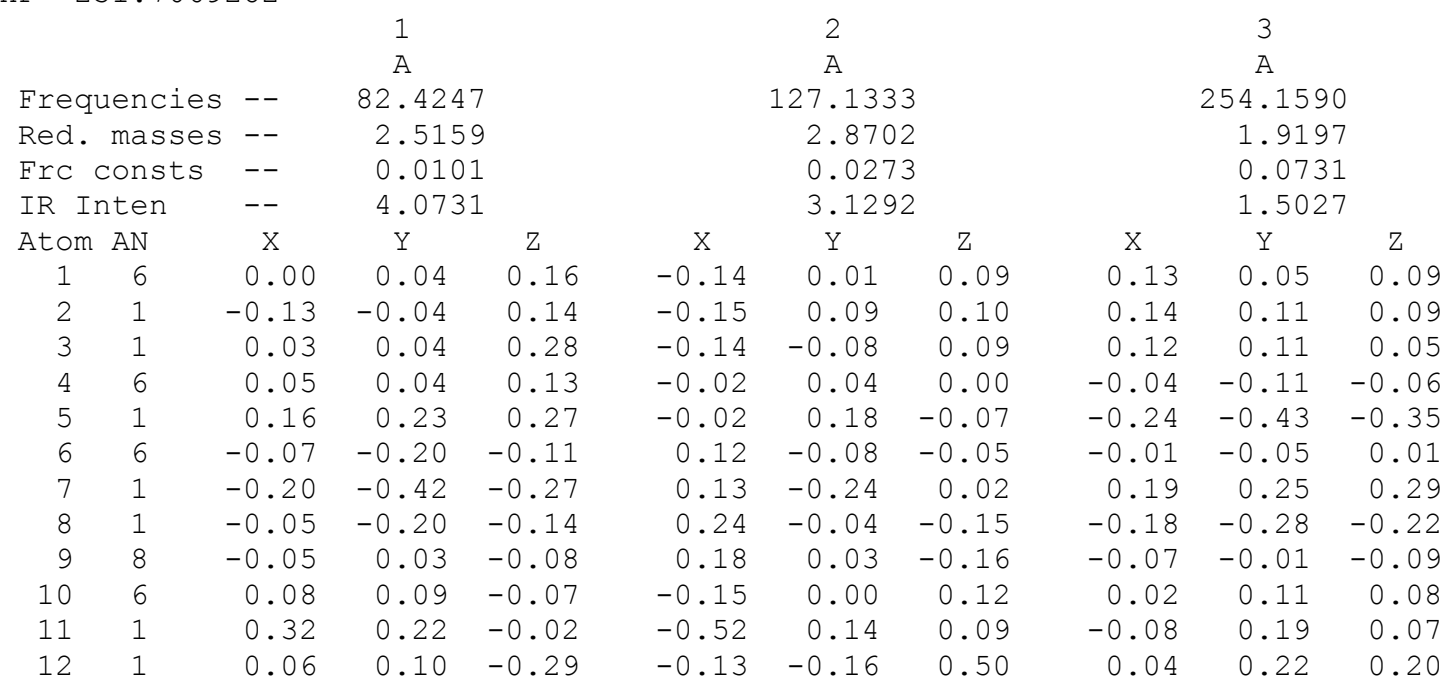

thermodynamics:

Sum of electronic and zero-point Energies= Sum of electronic and thermal Enthalpies=

Sum of electronic and thermal Free Energies=

$-231.609059$

$-231.602120$

$-231.638425$

Total free energy in solution:

with all non electrostatic terms

$($ a.u. $)=-231.792499$

III. Lewis acid mechanism

\section{$\mathbf{S} \sim \mathbf{P} \sim \boldsymbol{S}$ complex}




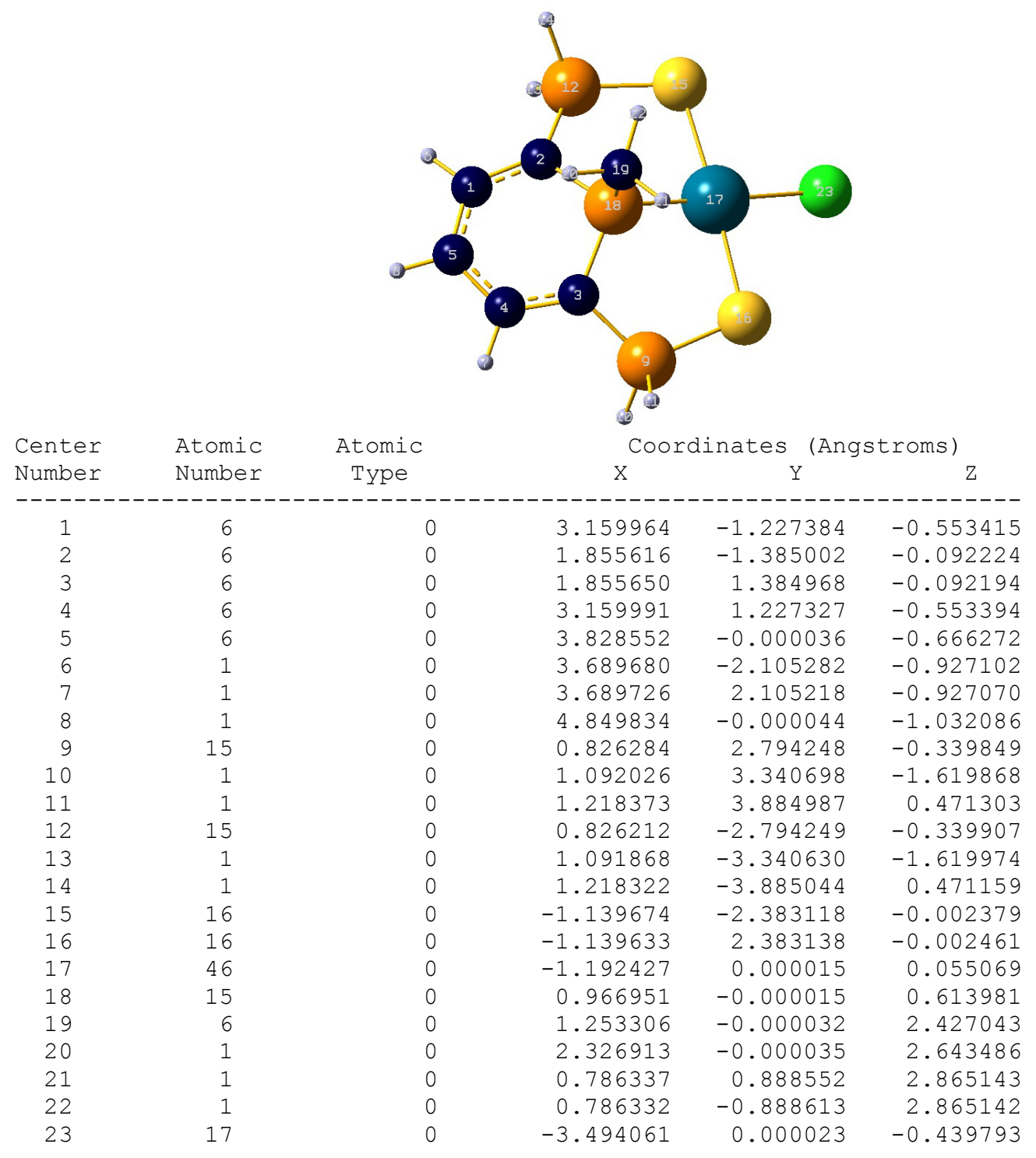

$\mathrm{HF}=-2641.755403$

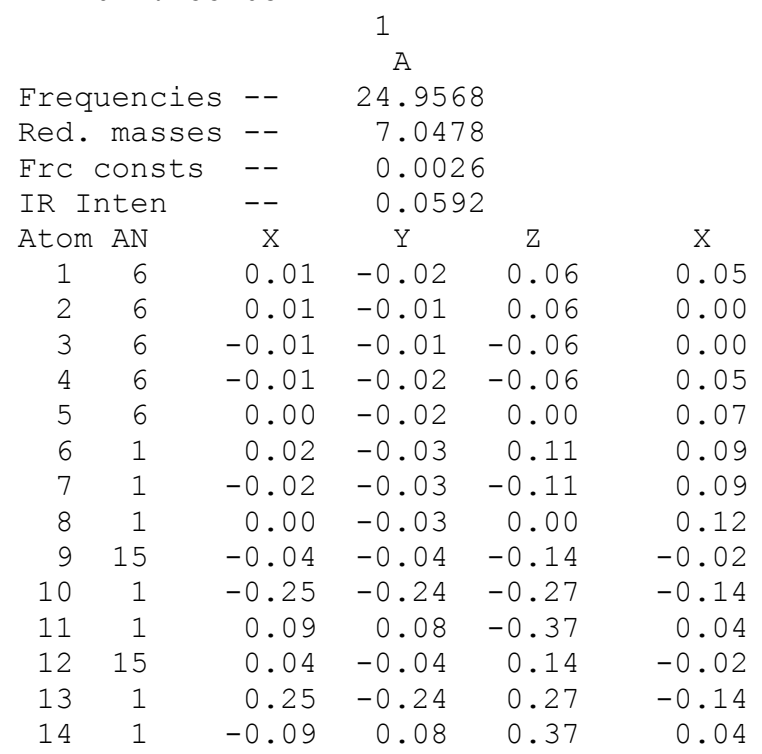

\section{2}

36.3834

4.3171

0.0034

6.1374

$\mathrm{Y}$

0.00

$-0.01-0.01$

$.01-0.01$

$0.00 \quad 0.13$

$0.00 \quad 0.23$

$\begin{array}{ll}0.01 & 0.17\end{array}$

$\begin{array}{ll}-0.01 & 0.17\end{array}$

$0.00 \quad 0.36$

$-0.05-0.17$

$-0.24-0.28$

$0.08-0.37$

$\begin{array}{lll}0.05 & -0.17\end{array}$

$0.24-0.28$

$\begin{array}{ll}-0.08 & -0.37\end{array}$
3

A

55.0292

13.1789

0.0235

0.8834

Y Z

$0.00-0.10$

$-0.06$

$-0.03$

$-0.03$

$-0.06$

$-0.06$

$-0.09$

$0.00-0.02$

$0.00-0.02$

$0.00-0.10$

$0.00-0.13$

$0.00-0.15$

$\begin{array}{lll}-0.09 & 0.00 & -0.15 \\ -0.08 & 0.00 & -0.19\end{array}$

$\begin{array}{lll}-0.06 & -0.03 & -0.05\end{array}$

$\begin{array}{lll}-0.23 & -0.17 & -0.14\end{array}$

$\begin{array}{llll}0.03 & 0.06 & -0.22\end{array}$

$\begin{array}{lll}-0.06 & 0.03 & -0.05\end{array}$

$\begin{array}{lll}-0.23 & 0.17 & -0.14\end{array}$

$\begin{array}{lll}0.03 & -0.06 & -0.22\end{array}$ 


$\begin{array}{rrrrrrrrrrr}15 & 16 & -0.02 & 0.02 & -0.26 & 0.01 & 0.00 & 0.04 & -0.01 & -0.01 & 0.25 \\ 16 & 16 & 0.02 & 0.02 & 0.26 & 0.01 & 0.00 & 0.04 & -0.01 & 0.01 & 0.25 \\ 17 & 46 & 0.00 & 0.00 & 0.00 & -0.01 & 0.00 & 0.01 & 0.01 & 0.00 & 0.04 \\ 18 & 15 & 0.00 & 0.02 & 0.00 & 0.00 & 0.00 & -0.01 & 0.02 & 0.00 & 0.06 \\ 19 & 6 & 0.00 & 0.11 & 0.00 & 0.03 & 0.00 & -0.01 & 0.18 & 0.00 & 0.03 \\ 20 & 1 & 0.00 & 0.13 & 0.00 & 0.04 & 0.00 & -0.04 & 0.20 & 0.00 & -0.07 \\ 21 & 1 & -0.01 & 0.13 & -0.05 & 0.04 & 0.00 & -0.01 & 0.23 & 0.00 & 0.08 \\ 22 & 1 & 0.01 & 0.13 & 0.05 & 0.04 & 0.00 & -0.01 & 0.23 & 0.00 & 0.08 \\ 23 & 17 & 0.00 & 0.00 & 0.00 & -0.02 & 0.00 & 0.06 & 0.12 & 0.00 & -0.42\end{array}$

thermodynamics:

Sum of electronic and zero-point Energies=

$-2641.607610$

Sum of electronic and thermal Enthalpies=

$-2641.589958$

Sum of electronic and thermal Free Energies=

$-2641.654028$

Total free energy in solution:

with all non electrostatic terms

$(\mathrm{a} \cdot \mathrm{u} \cdot)=-2641.777854$

SCF energy, thermochemistry and PCM energy for $\mathbf{C l}^{-}$.



Optimized geometry, SCF energy, three lower frequencies, thermochemistry and PCM energy for B1.






\begin{tabular}{|c|c|c|c|c|c|}
\hline 1 & 6 & 0 & 1.222090 & 2.814983 & -0.377150 \\
\hline 2 & 6 & 0 & 1.384436 & 1.503130 & 0.074259 \\
\hline 3 & 6 & 0 & -1.385102 & 1.502542 & 0.074156 \\
\hline 4 & 6 & 0 & -1.223301 & 2.814486 & -0.377138 \\
\hline 5 & 6 & 0 & -0.000735 & 3.478705 & -0.506304 \\
\hline 6 & 1 & 0 & 2.108931 & 3.350634 & -0.716283 \\
\hline 7 & 1 & 0 & -2.110367 & 3.349796 & -0.716223 \\
\hline 8 & 1 & 0 & -0.000942 & 4.499830 & -0.870220 \\
\hline 9 & 15 & 0 & -2.838139 & 0.520711 & -0.198537 \\
\hline 10 & 1 & 0 & -3.422439 & 0.950181 & -1.409387 \\
\hline 11 & 1 & 0 & -3.864040 & 0.788030 & 0.729968 \\
\hline 12 & 15 & 0 & 2.837774 & 0.521836 & -0.198859 \\
\hline 13 & 1 & 0 & 3.420986 & 0.950989 & -1.410346 \\
\hline 14 & 1 & 0 & 3.864163 & 0.790186 & 0.728798 \\
\hline 15 & 16 & 0 & 2.387531 & -1.459601 & -0.117516 \\
\hline 16 & 16 & 0 & -2.386753 & -1.460507 & -0.118332 \\
\hline 17 & 46 & 0 & 0.000420 & -1.456258 & -0.271173 \\
\hline 18 & 15 & 0 & -0.000136 & 0.552226 & 0.634237 \\
\hline 19 & 6 & 0 & -0.000490 & 0.288055 & 2.439757 \\
\hline 20 & 1 & 0 & -0.000797 & 1.261221 & 2.943262 \\
\hline 21 & 1 & 0 & -0.890152 & -0.282202 & 2.724608 \\
\hline 22 & 1 & 0 & 0.889021 & -0.282080 & 2.725259 \\
\hline
\end{tabular}

$\mathrm{HF}=-2181.3612993$

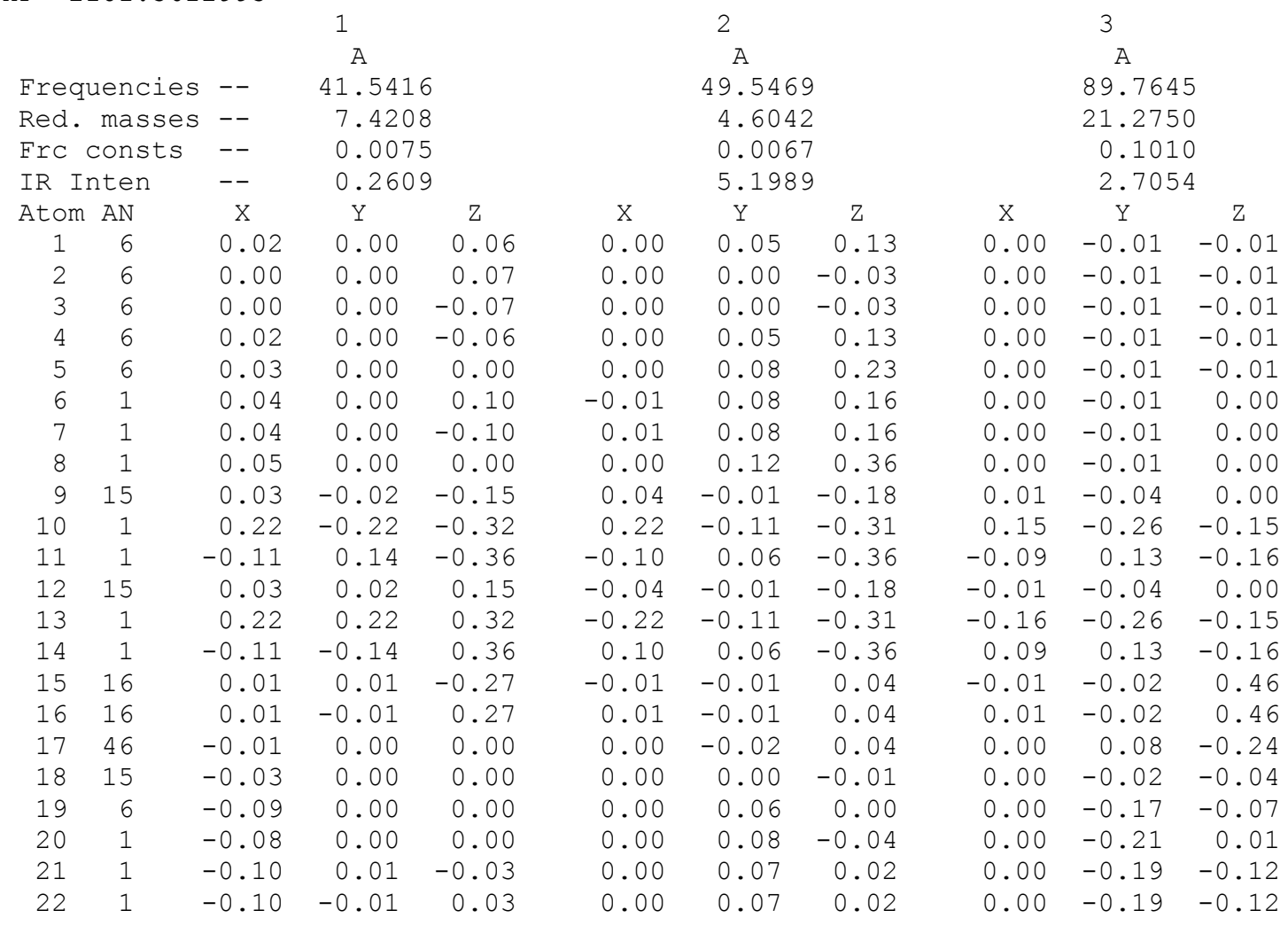

\footnotetext{
thermodynamics:

Sum of electronic and zero-point Energies=

$-2181.213620$

Sum of electronic and thermal Enthalpies=

$-2181.198011$

Sum of electronic and thermal Free Energies=

$-2181.256436$

Total free energy in solution:

with all non electrostatic terms

$\left(\mathrm{a} \cdot \mathrm{u}_{0}\right)=-2181.433710$
}

Optimized geometry, SCF energy, three lower frequencies, thermochemistry and PCM energy for $\mathbf{B 2}$. 


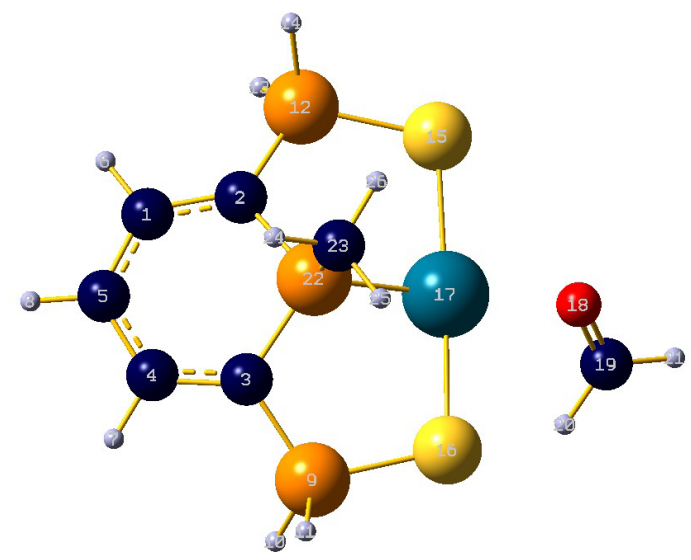

\begin{tabular}{|c|c|c|c|c|c|}
\hline \multirow{2}{*}{$\begin{array}{l}\text { Center } \\
\text { Number }\end{array}$} & \multirow{2}{*}{$\begin{array}{l}\text { Atomic } \\
\text { Number }\end{array}$} & \multirow{2}{*}{$\begin{array}{l}\text { Atomic } \\
\text { Type }\end{array}$} & \multicolumn{3}{|c|}{ Coordinates (Angstroms) } \\
\hline & & & $x$ & Y & Z \\
\hline 1 & 6 & 0 & 3.240252 & -0.789851 & -0.734257 \\
\hline 2 & 6 & 0 & 2.022765 & -1.133355 & -0.146810 \\
\hline 3 & 6 & 0 & 1.627916 & 1.603959 & -0.113846 \\
\hline 4 & 6 & 0 & 2.894644 & 1.632181 & -0.703778 \\
\hline 5 & 6 & 0 & 3.707489 & 0.516476 & -0.916839 \\
\hline 6 & 1 & 0 & 3.855545 & -1.590664 & -1.145113 \\
\hline 7 & 1 & 0 & 3.260518 & 2.584437 & -1.088462 \\
\hline 8 & 1 & 0 & 4.673773 & 0.660488 & -1.386618 \\
\hline 9 & 15 & 0 & 0.437577 & 2.897883 & -0.209473 \\
\hline 10 & 1 & 0 & 0.551945 & 3.544683 & -1.460250 \\
\hline 11 & 1 & 0 & 0.684646 & 3.965096 & 0.678349 \\
\hline 12 & 15 & 0 & 1.239995 & -2.709279 & -0.273422 \\
\hline 13 & 1 & 0 & 1.562566 & -3.276894 & -1.525842 \\
\hline 14 & 1 & 0 & 1.773130 & -3.668690 & 0.612148 \\
\hline 15 & 16 & 0 & -0.760118 & -2.549703 & 0.088585 \\
\hline 16 & 16 & 0 & -1.430058 & 2.179618 & 0.198375 \\
\hline 17 & 46 & 0 & -1.160064 & -0.191651 & 0.091056 \\
\hline 18 & 8 & 0 & -3.288182 & -0.552313 & -0.321023 \\
\hline 19 & 6 & 0 & -4.018345 & 0.015789 & -1.115626 \\
\hline 20 & 1 & 0 & -3.665881 & 0.855777 & -1.733426 \\
\hline 21 & 1 & 0 & -5.062767 & -0.310445 & -1.221846 \\
\hline 22 & 15 & 0 & 0.977277 & 0.104388 & 0.592229 \\
\hline 23 & 6 & 0 & 1.146719 & 0.103257 & 2.412381 \\
\hline 24 & 1 & 0 & 2.198111 & 0.249521 & 2.682627 \\
\hline 25 & 1 & 0 & 0.538476 & 0.908485 & 2.837214 \\
\hline 26 & 1 & 0 & 0.789797 & -0.851582 & 2.812134 \\
\hline
\end{tabular}

$\mathrm{HF}=-2295.847417$

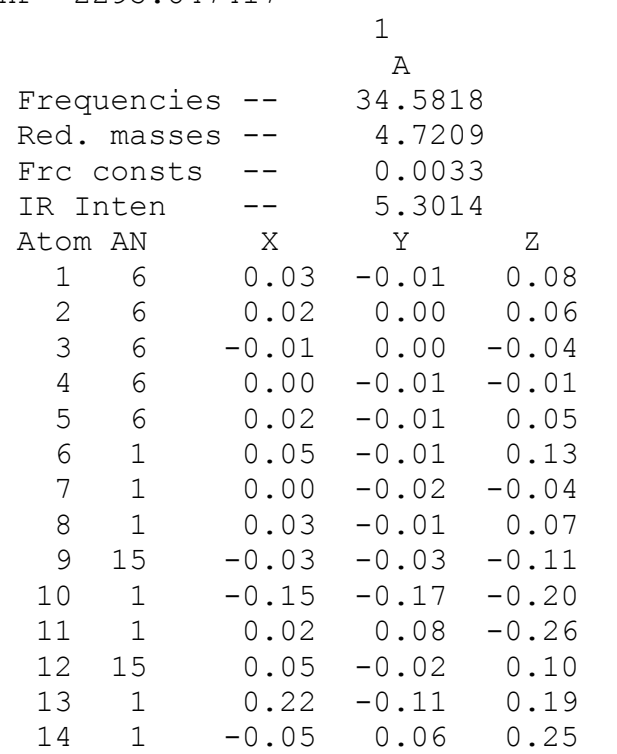

$\begin{array}{ll}2 & \\ A & \\ 39.9311 & \\ 4.7602 & \\ 0.0045 & \\ 1.1535 & \\ Y & Z \\ 0.01 & 0.12 \\ 0.00 & -0.02 \\ 0.00 & 0.02 \\ 0.01 & 0.16 \\ 0.01 & 0.23 \\ 0.02 & 0.15 \\ 0.02 & 0.20 \\ 0.02 & 0.35 \\ -0.02 & -0.10 \\ -0.09 & -0.13 \\ 0.04 & -0.16 \\ 0.03 & -0.17 \\ 0.19 & -0.27 \\ -0.09 & -0.35\end{array}$

3 A 54.0076 4.3363 0.0075 12.1726 $\begin{array}{ccc}X & Y & Z \\ -0.02 & 0.01 & -0.05\end{array}$ $-0.05$ $\begin{array}{lll}0.01 & 0.00 & 0.01\end{array}$ $\begin{array}{lll}0.02 & 0.01 & 0.04\end{array}$ $\begin{array}{lll}-0.01 & 0.01 & -0.02\end{array}$ $\begin{array}{llll}-0.03 & 0.01 & -0.09\end{array}$ $\begin{array}{lll}-0.03 & 0.01 & -0.07\end{array}$ $\begin{array}{lll}-0.03 & 0.02 & -0.01\end{array}$ $\begin{array}{lll}-0.06 & 0.01 & -0.14\end{array}$ $\begin{array}{lll}0.04 & 0.04 & 0.15\end{array}$ $\begin{array}{lll}0.18 & 0.23 & 0.26\end{array}$ $\begin{array}{lll}-0.03 & -0.10 & 0.33\end{array}$ $\begin{array}{lll}0.03 & -0.02 & 0.08\end{array}$ $\begin{array}{lll}0.06 & -0.07 & 0.11\end{array}$ $\begin{array}{lll}0.04 & 0.04 & 0.13\end{array}$ 


$\begin{array}{rrrrrrrrrrr}15 & 16 & 0.00 & -0.01 & -0.19 & 0.01 & 0.01 & 0.06 & 0.03 & -0.01 & 0.06 \\ 16 & 16 & 0.01 & -0.01 & 0.13 & 0.00 & 0.00 & -0.10 & -0.01 & 0.00 & -0.14 \\ 17 & 46 & 0.00 & -0.01 & -0.02 & -0.01 & 0.00 & -0.01 & 0.01 & -0.01 & -0.06 \\ 18 & 8 & 0.00 & -0.02 & -0.02 & -0.02 & -0.03 & 0.12 & 0.00 & -0.05 & -0.03 \\ 19 & 6 & -0.09 & 0.17 & 0.20 & -0.12 & -0.02 & 0.21 & -0.14 & 0.12 & 0.22 \\ 20 & 1 & -0.18 & 0.38 & 0.43 & -0.20 & 0.02 & 0.21 & -0.28 & 0.32 & 0.42 \\ 21 & 1 & -0.08 & 0.14 & 0.18 & -0.12 & -0.04 & 0.30 & -0.13 & 0.07 & 0.26 \\ 22 & 15 & -0.01 & 0.01 & 0.00 & -0.01 & -0.01 & -0.01 & 0.00 & -0.01 & -0.01 \\ 23 & 6 & -0.04 & 0.07 & 0.00 & -0.01 & -0.03 & -0.01 & -0.08 & -0.04 & 0.00 \\ 24 & 1 & -0.04 & 0.09 & 0.01 & -0.01 & -0.03 & -0.01 & -0.10 & -0.04 & 0.05 \\ 25 & 1 & -0.05 & 0.08 & -0.04 & -0.01 & -0.03 & 0.00 & -0.11 & -0.05 & -0.01 \\ 26 & 1 & -0.04 & 0.08 & 0.03 & -0.01 & -0.03 & -0.03 & -0.10 & -0.05 & -0.03\end{array}$

thermodynamics:

Sum of electronic and zero-point Energies=

$-2295.670246$

Sum of electronic and thermal Enthalpies=

$-2295.650833$

Sum of electronic and thermal Free Energies=

$-2295.718383$

Total free energy in solution:

with all non electrostatic terms

$(\mathrm{a} \cdot \mathrm{u})=$.

Optimized geometry, SCF energy, three lower frequencies, thermochemistry and PCM energy for $\mathbf{T S}_{\mathbf{B} 2 \mathbf{B} 3}$.

\begin{tabular}{|c|c|c|c|c|c|}
\hline Center & Atomic & Atomic & \multicolumn{3}{|c|}{ Coordinates (Angstroms) } \\
\hline Number & Number & Type & $\mathrm{x}$ & Y & Z \\
\hline 1 & 6 & 0 & -4.233240 & 1.378484 & -1.931912 \\
\hline 2 & 6 & 0 & -3.316452 & 1.513487 & -0.887203 \\
\hline 3 & 6 & 0 & -3.721022 & -1.209284 & -0.603546 \\
\hline 4 & 6 & 0 & -4.590396 & -1.030732 & -1.679477 \\
\hline 5 & 6 & 0 & -4.912844 & 0.201547 & -2.258688 \\
\hline 6 & 1 & 0 & -4.393017 & 2.233655 & -2.589040 \\
\hline 7 & 1 & 0 & -5.008368 & -1.918292 & -2.155234 \\
\hline 8 & 1 & 0 & -5.602488 & 0.216257 & -3.095016 \\
\hline 9 & 15 & 0 & -3.002061 & -2.741453 & -0.112160 \\
\hline 10 & 1 & 0 & -2.788579 & -3.539423 & -1.258442 \\
\hline 11 & 1 & 0 & -3.883524 & -3.557649 & 0.627699 \\
\hline 12 & 15 & 0 & -2.170108 & 2.835251 & -0.702780 \\
\hline 13 & 1 & 0 & -1.802396 & 3.310870 & -1.981327 \\
\hline 14 & 1 & 0 & -2.734474 & 3.996301 & -0.133158 \\
\hline 15 & 16 & 0 & -0.588518 & 2.266207 & 0.457353 \\
\hline 16 & 16 & 0 & -1.328059 & -2.425936 & 1.009450 \\
\hline 17 & 46 & 0 & -0.881806 & -0.090516 & 0.745836 \\
\hline 18 & 8 & 0 & 1.176758 & -0.478948 & 1.262159 \\
\hline 19 & 6 & 0 & 1.650006 & -0.346086 & 2.414733 \\
\hline 20 & 1 & 0 & 1.030201 & 0.001158 & 3.249776 \\
\hline 21 & 1 & 0 & 2.564769 & -0.887539 & 2.673186 \\
\hline 22 & 15 & 0 & -3.029694 & 0.185159 & 0.265145 \\
\hline 23 & 6 & 0 & -3.945451 & 0.480978 & 1.822372 \\
\hline 24 & 1 & 0 & -5.010982 & 0.611335 & 1.604837 \\
\hline
\end{tabular}




25
26
27
28
29
30
31
32
33
34
35
36
37
38
39
40
41
42
43
44
45
46
47

$$
\begin{array}{r}
-3.806098 \\
-3.555653 \\
2.833567 \\
2.644397 \\
1.744999 \\
3.645477 \\
4.587738 \\
3.568489 \\
4.480258 \\
2.693841 \\
3.632907 \\
4.002775 \\
1.870552 \\
5.336634 \\
3.074025 \\
4.444647 \\
4.710815 \\
1.993540 \\
0.990317 \\
1.708185 \\
6.250799 \\
5.481509 \\
5.199689
\end{array}
$$

2.494716

2. 313284

\begin{tabular}{|c|c|c|c|c|}
\hline \multicolumn{5}{|c|}{1} \\
\hline \multicolumn{2}{|c|}{ Frequencies } & $5--$ & -49.4892 & \\
\hline Red. & masses & $5--$ & 7.9860 & \\
\hline Frc & consts & -- & 0.0115 & \\
\hline IR I & nten & -- & 60.7530 & \\
\hline Atom & AN & $\mathrm{X}$ & $\mathrm{Y}$ & Z \\
\hline 1 & 6 & 0.00 & -0.02 & 0.03 \\
\hline 2 & 6 & 0.01 & -0.01 & 0.01 \\
\hline 3 & 6 & 0.05 & -0.01 & 0.03 \\
\hline 4 & 6 & 0.03 & -0.03 & 0.04 \\
\hline 5 & 6 & 0.01 & -0.03 & 0.04 \\
\hline 6 & 1 & -0.03 & -0.03 & 0.03 \\
\hline 7 & 1 & 0.03 & -0.03 & 0.05 \\
\hline 8 & 1 & -0.01 & -0.04 & 0.05 \\
\hline 9 & 15 & 0.07 & 0.00 & 0.03 \\
\hline 10 & 1 & 0.03 & -0.02 & 0.04 \\
\hline 11 & 1 & 0.10 & 0.01 & 0.07 \\
\hline 12 & 15 & -0.01 & 0.01 & 0.00 \\
\hline 13 & 1 & -0.07 & 0.05 & -0.01 \\
\hline 14 & 1 & -0.02 & -0.01 & 0.04 \\
\hline 15 & 16 & 0.04 & 0.03 & -0.07 \\
\hline 16 & 16 & 0.11 & 0.02 & -0.03 \\
\hline 17 & 46 & 0.07 & 0.03 & -0.02 \\
\hline 18 & 8 & 0.07 & 0.12 & -0.13 \\
\hline 19 & 6 & 0.03 & 0.15 & -0.07 \\
\hline 20 & 1 & -0.12 & -0.11 & -0.07 \\
\hline 21 & 1 & -0.08 & -0.02 & 0.00 \\
\hline 22 & 15 & 0.05 & 0.00 & 0.02 \\
\hline 23 & 6 & 0.04 & 0.00 & 0.01 \\
\hline 24 & 1 & 0.04 & -0.02 & 0.01 \\
\hline 25 & 1 & 0.05 & 0.00 & 0.02 \\
\hline 26 & 1 & 0.03 & 0.00 & 0.01 \\
\hline 27 & 1 & -0.28 & -0.36 & -0.04 \\
\hline 28 & 6 & -0.28 & -0.38 & -0.04 \\
\hline 29 & 1 & -0.22 & -0.29 & -0.04 \\
\hline 30 & 6 & -0.12 & -0.08 & 0.00 \\
\hline 31 & 1 & -0.11 & -0.01 & 0.04 \\
\hline 32 & 6 & -0.03 & 0.03 & 0.06 \\
\hline 33 & 1 & 0.05 & -0.11 & 0.07 \\
\hline 34 & 1 & 0.02 & 0.06 & -0.01 \\
\hline 35 & 50 & -0.09 & -0.03 & 0.03 \\
\hline 36 & 6 & -0.08 & -0.02 & 0.02 \\
\hline
\end{tabular}

3.725621

2. 656770

2.350737

1.763798

2.162193

0.317967

$-0.070318$

$-0.008940$

$-0.870200$

0.348260

$-2.089764$

$-2.144222$

0.778313

$-0.274179$

1.154054

$-2.817723$

$-1.475037$

$-2.639387$

$-1.554123$

$-2.816977$

$-2.757721$
$\mathrm{HF}=-2536.1991598$$$
2
$$

A

13.4354

5.7857

0.0006

0.2312

$x$

0.04

0.05

$-0.03$

$-0.02$

$-0.05$

$-0.05$

$-0.01$

0.07

$-0.09$

$-0.01$

$-0.11$

$-0.19$

$-0.13$

0.10

0.15

0.14

0.05

$-0.06$

0.00

0.00

0.00

0.00

0.00

0.01

0.02

0.03

0.00

0.06

$-0.02$

$-0.01$

$-0.01$

$-0.01$

$-0.01$

0.00

0.00

0.00

0.00

0.03
0.01

0.00

$-0.02$

$-0.05$

$-0.01$

$-0.03$

0.00

$-0.07$

0.09

$-0.05$

$-0.15$

0.01

$-0.05$

$-0.02$

$-0.04$

$-0.08$

$-0.14$

$-0.19$

$-0.15$

0.02

0.12

0.15

0.15

0.13

$-0.19$

$-0.13$

$-0.12$

$-0.09$

$-0.11$

0.00

0.02

0.02

$0.07-0.01$

$0.00-0.10$
3

A

17.5733

6.7228

0.0012

0.3288

X

0.17

0.10

0.09

0.16

0.19

0.21

0.19

0.25

0.06

0.11

0.02

0.08

0.14

0.04

0.02

0.00

0.02

0.01

0.03

0.06

0.04

0.04

$-0.04$

$-0.03$

$-0.07$

$-0.06$

0.12

0.08

0.07

0.03

0.04

$-0.03$

$-0.04$

$-0.03$

$-0.10$

$-0.05$

0.01

$$
\mathrm{Y}
$$

$-0.02$

$-0.01$

$-0.01$

$-0.02$

$-0.03$

$-0.03$

$-0.03$

$-0.04$

$-0.01$

$-0.02$

0.00

$-0.01$

0.00

$-0.01$

0.00

0.00

0.00

$-0.01$

0.00

0.04

0.01

$-0.01$

0.00

0.00

0.01

0.01

0.03

0.03

0.04

0.00

$-0.02$

0.00

$-0.01$

0.01

$\begin{array}{ll}0.01 & -0.02 \\ 0.00 & -0.06\end{array}$ 


$\begin{array}{rrrrrrrrrrr}37 & 6 & -0.05 & 0.07 & -0.03 & 0.00 & 0.11 & -0.01 & -0.16 & 0.02 & 0.06 \\ 38 & 6 & -0.05 & 0.06 & 0.11 & 0.00 & 0.15 & 0.00 & -0.18 & 0.01 & -0.12 \\ 39 & 1 & -0.08 & 0.01 & 0.05 & 0.03 & -0.03 & -0.11 & -0.02 & 0.02 & 0.01 \\ 40 & 1 & -0.13 & -0.03 & 0.00 & 0.03 & 0.04 & -0.15 & -0.12 & -0.01 & -0.10 \\ 41 & 1 & -0.04 & -0.04 & -0.01 & 0.03 & -0.03 & -0.09 & 0.02 & -0.03 & -0.12 \\ 42 & 1 & -0.05 & 0.07 & -0.03 & 0.02 & 0.15 & -0.07 & -0.21 & 0.01 & 0.06 \\ 43 & 1 & -0.08 & 0.11 & -0.06 & 0.01 & 0.05 & -0.03 & -0.14 & 0.05 & 0.09 \\ 44 & 1 & 0.02 & 0.08 & -0.03 & -0.02 & 0.14 & 0.04 & -0.17 & 0.02 & 0.05 \\ 45 & 1 & -0.07 & 0.03 & 0.14 & 0.00 & 0.12 & 0.00 & -0.14 & -0.01 & -0.17 \\ 46 & 1 & -0.02 & 0.08 & 0.08 & 0.00 & 0.19 & -0.05 & -0.23 & 0.02 & -0.14 \\ 47 & 1 & -0.04 & 0.08 & 0.14 & -0.01 & 0.18 & 0.06 & -0.20 & 0.02 & -0.10\end{array}$

thermodynamics:

Sum of electronic and zero-point Energies=

$-2535.840124$

Sum of electronic and thermal Enthalpies=

$-2535.807838$

Sum of electronic and thermal Free Energies=

$-2535.906665$

Total free energy in solution:

with all non electrostatic terms

$\left(\mathrm{a} \cdot \mathrm{u}_{\text {. }}\right)=-2536.225922$

Optimized geometry, SCF energy, three lower frequencies, thermochemistry and PCM energy for B3.



\begin{tabular}{|c|c|c|c|c|c|}
\hline \multirow{2}{*}{$\begin{array}{l}\text { Center } \\
\text { Number }\end{array}$} & \multirow{2}{*}{$\begin{array}{l}\text { Atomic } \\
\text { Number }\end{array}$} & \multirow{2}{*}{$\begin{array}{l}\text { Atomic } \\
\text { Type }\end{array}$} & \multicolumn{3}{|c|}{ Coordinates (Angstroms) } \\
\hline & & & $\mathrm{X}$ & Y & Z \\
\hline 1 & 6 & 0 & 2.345451 & 0.812647 & 2.728241 \\
\hline 2 & 1 & 0 & 1.362341 & 1.302231 & 2.739693 \\
\hline 3 & 1 & 0 & 2.572667 & 0.537313 & 3.769935 \\
\hline 4 & 6 & 0 & 3.397857 & 1.755990 & 2.225247 \\
\hline 5 & 1 & 0 & 4.419955 & 1.370266 & 2.229760 \\
\hline 6 & 6 & 0 & 3.180756 & 3.007785 & 1.818896 \\
\hline 7 & 1 & 0 & 3.994033 & 3.651698 & 1.494406 \\
\hline 8 & 1 & 0 & 2.183064 & 3.442622 & 1.815156 \\
\hline 9 & 50 & 0 & 2.781975 & -0.630432 & -1.082399 \\
\hline 10 & 6 & 0 & 4.381505 & -1.912126 & -0.472193 \\
\hline 11 & 1 & 0 & 4.889764 & -2.290708 & -1.365781 \\
\hline 12 & 1 & 0 & 5.120887 & -1.381465 & 0.134522 \\
\hline 13 & 1 & 0 & 4.005824 & -2.770914 & 0.092127 \\
\hline 14 & 6 & 0 & 1.341336 & -1.603363 & -2.325418 \\
\hline 15 & 1 & 0 & 0.981836 & -2.516115 & -1.841775 \\
\hline 16 & 1 & 0 & 0.488430 & -0.946060 & -2.519767 \\
\hline 17 & 1 & 0 & 1.795709 & -1.871209 & -3.285474 \\
\hline 18 & 6 & 0 & 3.406354 & 1.284195 & -1.788153 \\
\hline 19 & 1 & 0 & 3.764733 & 1.887661 & -0.950202 \\
\hline 20 & 1 & 0 & 4.215806 & 1.168431 & -2.516538 \\
\hline 21 & 1 & 0 & 2.572409 & 1.806036 & -2.264920 \\
\hline 22 & 1 & 0 & 3.243994 & -0.954710 & 1.862029 \\
\hline 23 & 6 & 0 & 2.250428 & -0.493392 & 1.941910 \\
\hline 24 & 8 & 0 & 1.712427 & -0.310586 & 0.626468 \\
\hline 25 & 1 & 0 & 1.609396 & -1.204073 & 2.479664 \\
\hline
\end{tabular}




$\begin{array}{rr}26 & 1 \\ 27 & 15 \\ 28 & 6 \\ 29 & 1 \\ 30 & 16 \\ 31 & 6 \\ 32 & 46 \\ 33 & 6 \\ 34 & 1 \\ 35 & 6 \\ 36 & 16 \\ 37 & 6 \\ 38 & 1 \\ 39 & 15 \\ 40 & 1 \\ 41 & 1 \\ 42 & 1 \\ 43 & 15 \\ 44 & 6 \\ 45 & 1 \\ 46 & 1 \\ 47 & 1\end{array}$

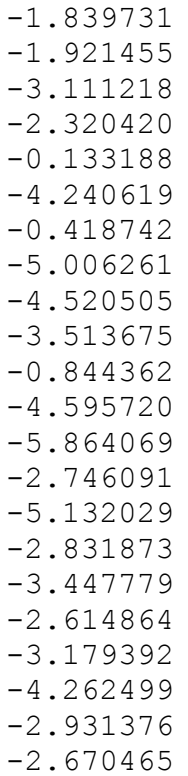

$-2.519864$

$-1.128948$

$-0.828835$

$-0.664565$

$-0.253294$

$-1.610037$

0.519497

$-1.565809$

$-2.361008$

0.022943

1.252239

$-0.856345$

$-2.223533$

0.620709

$-1.063334$

$-0.369158$

1.683703

0.462487

2.092014

2.069734

2.866654

2.328260

$\mathrm{HF}=-2536.2616829$

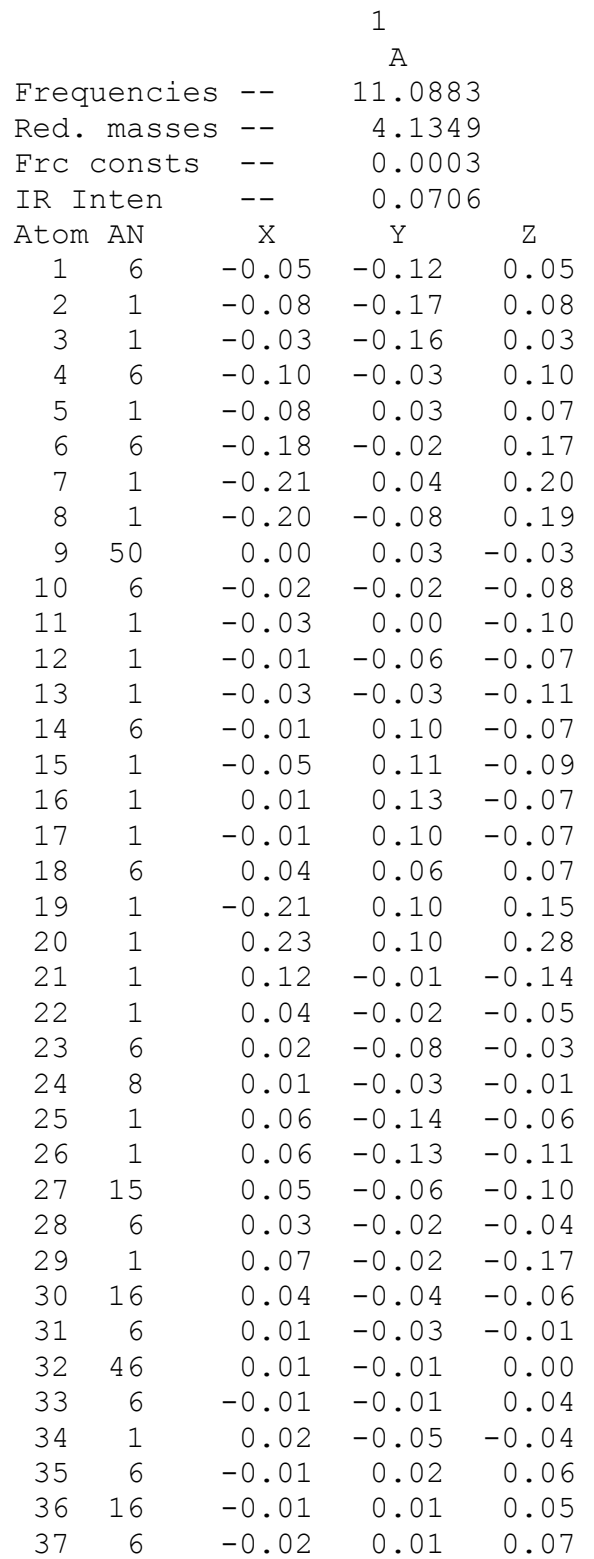

2

20.2746

2.7622

0.0007

0.1238

$\mathrm{X}$

$-0.03$

$\mathrm{Y}$

$0.04-0.01$

$-0.04$

$-0.05$

$-0.03$

$-0.03$

$-0.04$

$-0.04$

$-0.04$

0.02

$-0.11$

$-0.14$

$-0.06$

$-0.20$

$-0.03$

$-0.08$

0.01

$-0.04$

0.20

0.52

0.00

0.18

0.00

$-0.01$

0.00

0.00

$-0.06$

$-0.04$

$-0.03$

$-0.04$

$-0.02$

$-0.04$

0.00

$-0.03$

$-0.05$

0.00

0.02

$-0.01$
$0.03-0.03$

$0.05-0.01$

$0.05 \quad 0.00$

$\begin{array}{ll}0.06 & 0.02\end{array}$

$0.05-0.01$

$0.05 \quad 0.00$

$0.04 \quad-0.03$

$-0.01 \quad 0.01$

$\begin{array}{ll}-0.17 & 0.02\end{array}$

$-0.230 .03$

$\begin{array}{ll}-0.23 & 0.03\end{array}$

$-0.120 .03$

$0.15-0.06$

$0.14 \quad-0.12$

$0.21-0.03$

$0.18-0.08$

$\begin{array}{ll}-0.05 & 0.07\end{array}$

$\begin{array}{ll}-0.20 & 0.04\end{array}$

$-0.12-0.14$

0.150 .33

$0.05 \quad 0.00$

$0.04-0.01$

$0.02-0.02$

$0.03-0.01$

$\begin{array}{ll}0.01 & 0.01\end{array}$

$0.00 \quad 0.01$

$0.00 \quad 0.02$

$0.00 \quad 0.02$

$0.01-0.01$

$\begin{array}{rr}-0.01 \quad 0.04 \\ -0.01 & -0.02\end{array}$

$0.01-0.02$

$\begin{array}{ll}-0.01 & 0.04\end{array}$

$0.00 \quad 0.04$

$\begin{array}{ll}-0.01 & 0.01\end{array}$

$0.00-0.03$

$\begin{array}{rr}-0.01 & 0.03\end{array}$
3

23.7940

3.7684

0.0013

0.2233

$\mathrm{X}$

0.09

0.12

0.07

0.16

0.13

0.24

0.29

0.27

$-0.02$

$-0.05$

$-0.06$

$-0.04$

$-0.07$

$-0.05$

$-0.08$

$-0.03$

$-0.06$

0.03

0.33

$-0.18$

$-0.03$

$-0.04$

$-0.01$

$-0.01$

$-0.05$

0.05

0.00

0.01

$-0.02$

$-0.02$

0.04

$-0.01$

0.04

0.05

0.00

$-0.01$

0.03
$\mathrm{Y}$

Z

$\begin{array}{ll}-0.04 & 0.01\end{array}$

$0.03 \quad 0.01$

$\begin{array}{ll}-0.05 & 0.01\end{array}$

$-0.11 \quad 0.01$

$\begin{array}{ll}-0.18 & 0.04\end{array}$

$\begin{array}{ll}-0.11 & -0.02\end{array}$

$\begin{array}{ll}-0.17 & -0.02\end{array}$

$\begin{array}{ll}-0.04 & -0.04\end{array}$

$0.05 \quad 0.00$

$-0.01-0.04$

$0.01-0.05$

$-0.05-0.02$

$-0.02-0.07$
$-0.12-0.02$

$0.12 \quad-0.02$

$0.12-0.04$

$\begin{array}{ll}0.15 & -0.01\end{array}$

$0.12-0.03$

$0.04 \quad 0.03$

$\begin{array}{ll}-0.08 & -0.01\end{array}$

$0.00-0.19$

$\begin{array}{ll}0.18 & 0.30\end{array}$

$-0.10 \quad 0.00$

$\begin{array}{ll}-0.03 & 0.01\end{array}$

$0.01 \quad 0.02$

$\begin{array}{ll}0.01 & 0.02\end{array}$

$\begin{array}{ll}-0.07 & -0.05\end{array}$

$-0.03-0.05$

$-0.04-0.06$

$-0.03-0.10$

$\begin{array}{ll}0.00 & 0.02\end{array}$

$-0.07-0.09$

$\begin{array}{lll}0.00 & 0.02\end{array}$

$\begin{array}{ll}-0.07-0.08 & -0.08\end{array}$

$-0.09-0.11$

$\begin{array}{ll}-0.02 & 0.00\end{array}$

$0.00 \quad 0.03$

$-0.05-0.03$ 


$\begin{array}{rrrrrrrrrrr}38 & 1 & -0.02 & -0.02 & 0.05 & -0.04 & -0.01 & 0.05 & 0.07 & -0.10 & -0.11 \\ 39 & 15 & -0.03 & 0.02 & 0.10 & 0.01 & -0.01 & 0.00 & -0.02 & 0.00 & 0.07 \\ 40 & 1 & -0.04 & 0.01 & 0.11 & -0.01 & -0.01 & 0.03 & 0.04 & -0.06 & -0.02 \\ 41 & 1 & -0.06 & -0.01 & 0.13 & 0.01 & -0.01 & 0.00 & -0.04 & -0.04 & 0.11 \\ 42 & 1 & -0.02 & 0.07 & 0.13 & 0.03 & -0.01 & 0.01 & -0.02 & 0.06 & 0.10 \\ 43 & 15 & 0.02 & 0.02 & 0.00 & 0.00 & -0.01 & 0.01 & -0.02 & -0.01 & -0.02 \\ 44 & 6 & 0.03 & 0.09 & -0.02 & 0.01 & -0.02 & 0.02 & -0.05 & 0.03 & -0.04 \\ 45 & 1 & 0.04 & 0.10 & -0.02 & 0.01 & -0.03 & 0.03 & -0.05 & 0.03 & -0.07 \\ 46 & 1 & 0.02 & 0.11 & 0.01 & 0.03 & -0.02 & 0.01 & -0.06 & 0.06 & -0.02 \\ 47 & 1 & 0.05 & 0.08 & -0.05 & 0.01 & -0.02 & 0.02 & -0.05 & 0.04 & -0.06\end{array}$

thermodynamics:

Sum of electronic and zero-point Energiest

$-2535.899717$

Sum of electronic and thermal Enthalpies=

$-2535.867115$

Sum of electronic and thermal Free Energies=

$-2535.968056$

Total free energy in solution:

with all non electrostatic terms

$($ a.u. $)=-2536.287457$

\section{$\mathbf{S} \sim \mathbf{C} \sim \boldsymbol{S}$ complex}

Optimized geometry, SCF energy, three lower frequencies, thermochemistry and PCM energy for B0.

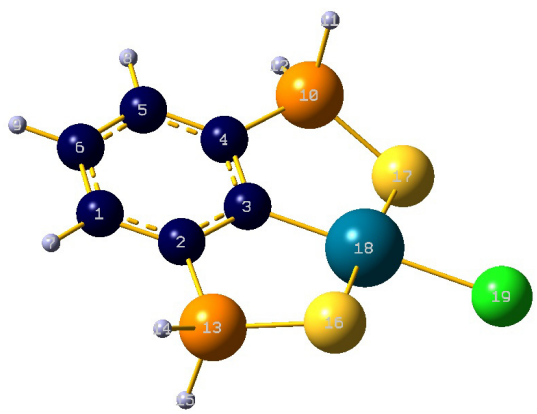

\begin{tabular}{rrrrrr}
$\begin{array}{c}\text { Center } \\
\text { Number }\end{array}$ & $\begin{array}{c}\text { Atomic } \\
\text { Number }\end{array}$ & $\begin{array}{c}\text { Atomic } \\
\text { Type }\end{array}$ & \multicolumn{3}{c}{ Coordinates (Angstroms) } \\
-1 & 6 & 0 & 3.227393 & -1.198228 & 0.110176 \\
1 & 6 & 0 & 1.829292 & -1.184511 & 0.106355 \\
2 & 6 & 0 & 1.063050 & -0.000133 & 0.000591 \\
3 & 6 & 0 & 1.829682 & 1.183903 & -0.105195 \\
4 & 6 & 0 & 3.227850 & 1.196938 & -0.110980 \\
5 & 6 & 0 & 3.931311 & -0.000794 & -0.000932 \\
6 & 1 & 0 & 3.775252 & -2.134393 & 0.197793 \\
7 & 1 & 0 & 3.776004 & 2.132901 & -0.198967 \\
8 & 1 & 0 & 5.017086 & -0.001102 & -0.001727 \\
9 & 15 & 0 & 0.827078 & 2.663527 & -0.264051 \\
10 & 1 & 0 & 0.883668 & 3.071972 & -1.616921 \\
11 & 1 & 0 & 1.514841 & 3.729409 & 0.359845 \\
12 & 15 & 0 & 0.825844 & -2.663548 & 0.264978 \\
13 & 1 & 0 & 1.513825 & -3.729851 & -0.357926 \\
14 & 1 & 0 & 0.880628 & -3.071729 & 1.617990 \\
15 & 16 & 0 & -1.025283 & -2.327857 & -0.434311 \\
16 & 16 & 0 & -1.024574 & 2.328352 & 0.433757 \\
17 & 46 & 0 & -0.951239 & 0.000137 & -0.000022 \\
18 & 17 & 0 & -3.308935 & 0.000342 & -0.000248 \\
19 & & 0 & & &
\end{tabular}

$\mathrm{HF}=-2298.6416932$

Frequencies -- $\quad 44.7490$

\section{2}

52.9969
3

67.8097 


\begin{tabular}{|c|c|c|c|c|c|c|c|c|c|c|}
\hline \multirow{2}{*}{\multicolumn{2}{|c|}{ Red. masses }} & $s--$ & \multicolumn{2}{|c|}{6.3466} & \multicolumn{3}{|c|}{8.9845} & \multicolumn{3}{|c|}{4.9832} \\
\hline & & -- & 0.007 & & & 0.01 & & & 0.013 & \\
\hline IR I & nten & -- & 0.978 & & & 2.04 & & & 4.220 & \\
\hline Atom & AN & $\mathrm{X}$ & $\mathrm{Y}$ & Z & $\mathrm{X}$ & $\mathrm{Y}$ & Z & $\mathrm{x}$ & $\mathrm{Y}$ & Z \\
\hline 1 & 6 & 0.00 & 0.00 & -0.09 & -0.01 & 0.01 & 0.20 & -0.02 & -0.02 & -0.05 \\
\hline 2 & 6 & 0.00 & -0.01 & -0.08 & -0.01 & 0.00 & 0.01 & -0.01 & 0.01 & 0.01 \\
\hline 3 & 6 & -0.01 & 0.00 & 0.00 & 0.00 & -0.01 & -0.06 & 0.00 & 0.02 & 0.00 \\
\hline 4 & 6 & 0.00 & 0.01 & 0.08 & 0.01 & 0.00 & 0.01 & 0.01 & 0.01 & 0.01 \\
\hline 5 & 6 & 0.00 & 0.00 & 0.09 & 0.01 & 0.01 & 0.20 & 0.02 & -0.02 & -0.05 \\
\hline 6 & 6 & 0.01 & 0.00 & 0.00 & 0.00 & 0.02 & 0.32 & 0.00 & -0.03 & -0.11 \\
\hline 7 & 1 & 0.00 & -0.01 & -0.16 & -0.01 & 0.02 & 0.27 & -0.03 & -0.03 & -0.06 \\
\hline 8 & 1 & 0.00 & 0.01 & 0.15 & 0.01 & 0.02 & 0.27 & 0.03 & -0.03 & -0.06 \\
\hline 9 & 1 & 0.01 & 0.00 & 0.00 & 0.00 & 0.03 & 0.48 & 0.00 & -0.05 & -0.17 \\
\hline 10 & 15 & 0.07 & 0.06 & 0.14 & 0.00 & -0.03 & -0.15 & 0.07 & 0.06 & 0.14 \\
\hline 11 & 1 & 0.32 & 0.26 & 0.21 & 0.01 & -0.16 & -0.19 & 0.26 & 0.32 & 0.23 \\
\hline 12 & 1 & 0.00 & -0.06 & 0.41 & -0.01 & 0.04 & -0.25 & 0.02 & -0.07 & 0.43 \\
\hline 13 & 15 & 0.07 & -0.06 & -0.14 & 0.00 & -0.03 & -0.15 & -0.07 & 0.06 & 0.14 \\
\hline 14 & 1 & 0.00 & 0.06 & -0.40 & 0.01 & 0.04 & -0.25 & -0.02 & -0.07 & 0.43 \\
\hline 15 & 1 & 0.32 & -0.26 & -0.21 & 0.00 & -0.17 & -0.19 & -0.26 & 0.32 & 0.23 \\
\hline 16 & 16 & -0.07 & -0.03 & 0.22 & 0.00 & 0.02 & -0.13 & 0.04 & -0.01 & -0.15 \\
\hline 17 & 16 & -0.07 & 0.03 & -0.22 & 0.00 & 0.02 & -0.13 & -0.04 & -0.01 & -0.15 \\
\hline 18 & 46 & -0.01 & 0.00 & 0.00 & 0.00 & 0.00 & -0.02 & 0.00 & -0.02 & -0.02 \\
\hline 19 & 17 & -0.01 & 0.00 & 0.00 & 0.00 & -0.01 & 0.32 & 0.00 & -0.04 & 0.12 \\
\hline
\end{tabular}

thermodynamics:

Sum of electronic and zero-point Energies=

$-2298.526999$

Sum of electronic and thermal Enthalpies=

$-2298.512427$

Sum of electronic and thermal Free Energies= Total free energy in solution:

with all non electrostatic terms

$-2298.569372$

$($ a.u. $)=-2298.670559$

Optimized geometry, SCF energy, three lower frequencies, thermochemistry and PCM energy for B1.

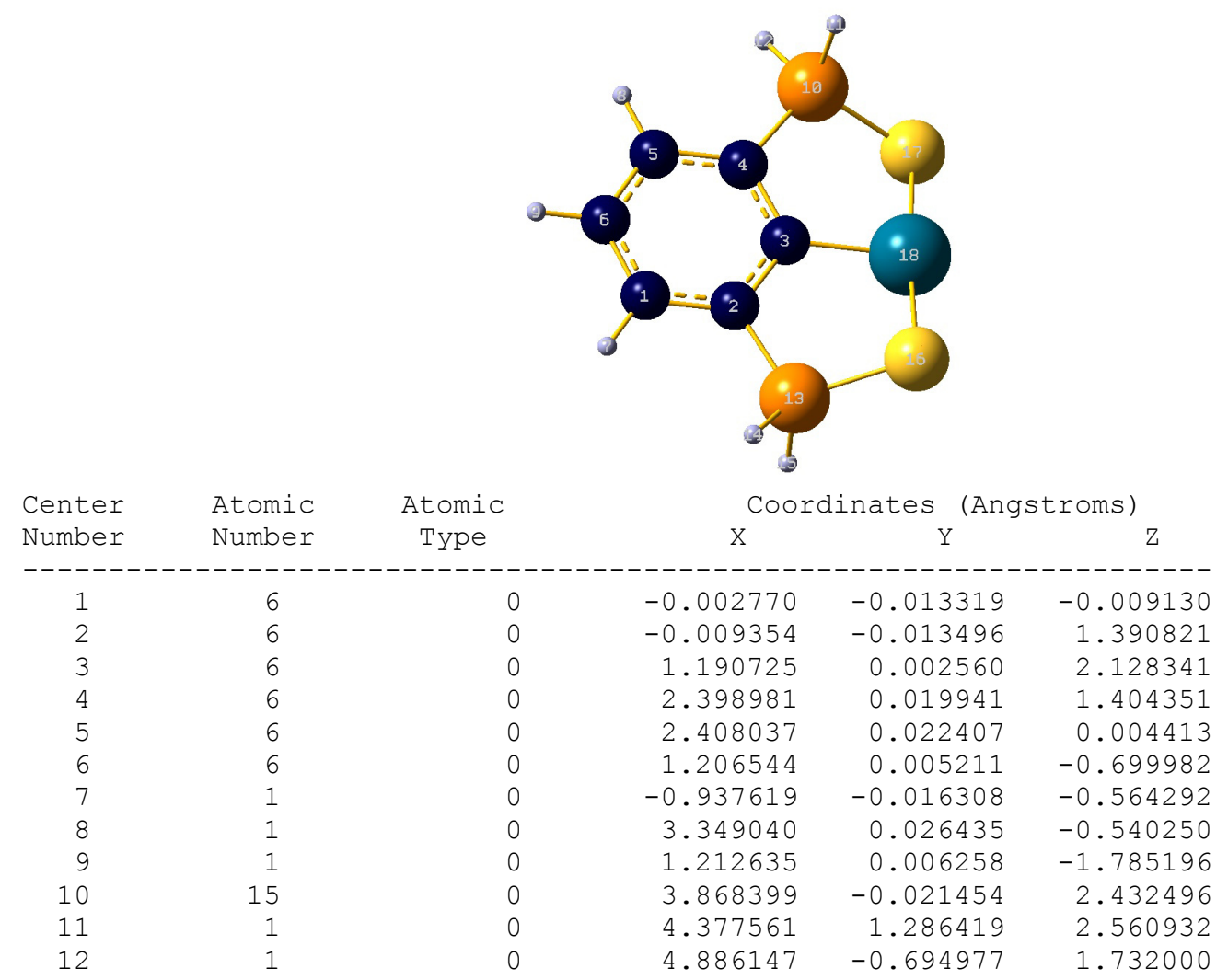




\begin{tabular}{|c|c|c|c|c|c|}
\hline 13 & 15 & 0 & -1.490177 & 0.026077 & 2.402577 \\
\hline 14 & 1 & 0 & -2.500123 & 0.700552 & 1.69181 \\
\hline 15 & 1 & 0 & -2.000479 & -1.282077 & 2.52330 \\
\hline 16 & 16 & 0 & -1.004691 & 0.910780 & 4.14988 \\
\hline 17 & 16 & 0 & 3.363476 & -0.908873 & 4.1728 \\
\hline 18 & 46 & 0 & 1.179767 & 0.001031 & 4.08648 \\
\hline
\end{tabular}

\begin{tabular}{|c|c|c|c|c|c|c|c|c|c|c|}
\hline & \multicolumn{2}{|l|}{1} & \multicolumn{3}{|c|}{2} & \multicolumn{3}{|c|}{3} \\
\hline & & & \multicolumn{2}{|l|}{ A } & \multicolumn{3}{|c|}{ A } & \multicolumn{3}{|c|}{ A } \\
\hline \multicolumn{2}{|c|}{ Frequencies } & -- & \multicolumn{2}{|c|}{67.7575} & \multicolumn{3}{|c|}{76.3756} & \multicolumn{3}{|c|}{103.1826} \\
\hline \multicolumn{2}{|c|}{ Red. masses } & -- & \multicolumn{2}{|c|}{7.0695} & \multicolumn{3}{|c|}{4.8515} & \multicolumn{3}{|c|}{17.6770} \\
\hline \multicolumn{2}{|c|}{ Frc consts } & -- & \multicolumn{2}{|c|}{0.0191} & \multicolumn{3}{|c|}{0.0167} & \multicolumn{3}{|c|}{0.1109} \\
\hline IR In & nten & -- & \multicolumn{2}{|c|}{2.3307} & \multicolumn{3}{|c|}{5.7171} & \multicolumn{3}{|c|}{0.5833} \\
\hline Atom & AN & $\mathrm{X}$ & $\mathrm{Y}$ & Z & $\mathrm{x}$ & $\mathrm{Y}$ & Z & $\mathrm{x}$ & $\mathrm{Y}$ & Z \\
\hline 1 & 6 & 0.00 & -0.11 & 0.01 & 0.00 & 0.11 & 0.01 & 0.04 & -0.09 & -0.01 \\
\hline 2 & 6 & 0.00 & -0.11 & 0.01 & 0.02 & -0.01 & 0.01 & 0.02 & 0.08 & -0.01 \\
\hline 3 & 6 & 0.00 & 0.00 & 0.00 & 0.02 & -0.02 & 0.00 & 0.01 & 0.15 & 0.00 \\
\hline 4 & 6 & 0.00 & 0.10 & 0.01 & 0.02 & -0.01 & -0.01 & 0.02 & 0.08 & 0.01 \\
\hline 5 & 6 & 0.00 & 0.11 & 0.01 & 0.00 & 0.11 & -0.01 & 0.04 & -0.09 & 0.01 \\
\hline 6 & 6 & 0.00 & 0.00 & 0.02 & -0.01 & 0.20 & 0.00 & 0.05 & -0.18 & 0.00 \\
\hline 7 & 1 & 0.00 & -0.20 & 0.01 & -0.01 & 0.14 & 0.02 & 0.05 & -0.16 & -0.02 \\
\hline 8 & 1 & 0.00 & 0.20 & 0.01 & -0.01 & 0.14 & -0.02 & 0.05 & -0.16 & 0.02 \\
\hline 9 & 1 & 0.00 & 0.00 & 0.02 & -0.02 & 0.32 & 0.00 & 0.06 & -0.32 & 0.00 \\
\hline 10 & 15 & -0.05 & 0.14 & 0.09 & 0.04 & -0.19 & -0.05 & -0.03 & 0.01 & 0.07 \\
\hline 11 & 1 & -0.22 & 0.18 & 0.34 & 0.28 & -0.27 & -0.14 & -0.12 & 0.02 & 0.35 \\
\hline 12 & 1 & 0.08 & 0.38 & 0.05 & -0.10 & -0.41 & -0.03 & 0.05 & 0.20 & 0.01 \\
\hline 13 & 15 & 0.05 & -0.14 & 0.09 & 0.04 & -0.19 & 0.05 & -0.03 & 0.01 & -0.07 \\
\hline 14 & 1 & -0.08 & -0.37 & 0.05 & -0.10 & -0.41 & 0.03 & 0.05 & 0.19 & 0.00 \\
\hline 15 & 1 & 0.21 & -0.18 & 0.34 & 0.28 & -0.27 & 0.15 & -0.11 & 0.02 & -0.35 \\
\hline 16 & 16 & 0.08 & 0.21 & -0.09 & -0.04 & 0.01 & -0.03 & -0.15 & -0.34 & 0.15 \\
\hline 17 & 16 & -0.08 & -0.21 & -0.09 & -0.05 & 0.00 & 0.03 & -0.15 & -0.35 & -0.15 \\
\hline 18 & 46 & 0.00 & 0.00 & -0.01 & 0.00 & 0.07 & 0.00 & 0.08 & 0.21 & 0.00 \\
\hline
\end{tabular}

\footnotetext{
thermodynamics:

Sum of electronic and zero-point Energies=

$-1838.126632$

Sum of electronic and thermal Enthalpies=

$-1838.114058$

Sum of electronic and thermal Free Energies=

$-1838.165695$

Total free energy in solution:

with all non electrostatic terms

$(\mathrm{a} \cdot \mathrm{u} \cdot)=-1838 \cdot 321593$
}

Optimized geometry, SCF energy, three lower frequencies, thermochemistry and PCM energy for B2.

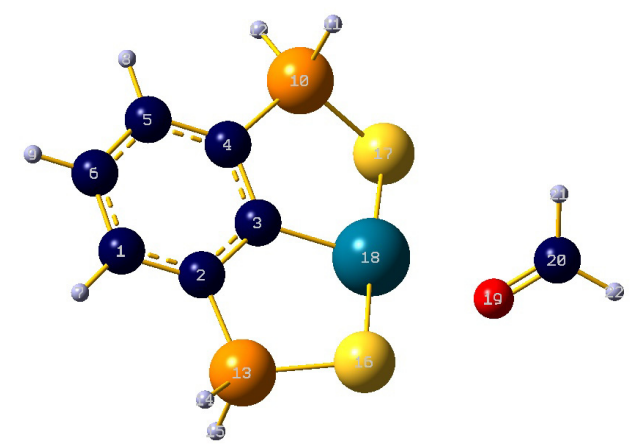

\begin{tabular}{cccrrr} 
Center & Atomic & Atomic & \multicolumn{2}{c}{ Coordinates (Angstroms) } \\
Number & Number & Type & $\mathrm{X}$ & $\mathrm{Y}$ & $\mathrm{Z}$ \\
--1 & 6 & 0 & 0.021099 & 0.056767 & -0.024225 \\
1 & 6 & 0 & -0.064593 & -0.064331 & 1.367298 \\
2 & 6 & 0 & 1.073684 & -0.070640 & 2.203413 \\
3 & 6 & 0 & 2.313374 & 0.057745 & 1.538388
\end{tabular}




\begin{tabular}{|c|c|c|c|c|c|}
\hline 5 & 6 & 0 & 2.416501 & 0.185247 & 0.148295 \\
\hline 6 & 6 & 0 & 1.265909 & 0.182942 & -0.635230 \\
\hline 7 & 1 & 0 & -0.877706 & 0.059603 & -0.636313 \\
\hline 8 & 1 & 0 & 3.388905 & 0.279314 & -0.329344 \\
\hline 9 & 1 & 0 & 1.338748 & 0.278657 & -1.713790 \\
\hline 10 & 15 & 0 & 3.734816 & 0.025216 & 2.626399 \\
\hline 11 & 1 & 0 & 4.194911 & 1.342802 & 2.828802 \\
\hline 12 & 1 & 0 & 4.816360 & -0.576135 & 1.954956 \\
\hline 13 & 15 & 0 & -1.617769 & -0.199635 & 2.250211 \\
\hline 14 & 1 & 0 & -2.604059 & 0.520434 & 1.549081 \\
\hline 15 & 1 & 0 & -2.082687 & -1.527627 & 2.157120 \\
\hline 16 & 16 & 0 & -1.329479 & 0.450839 & 4.133765 \\
\hline 17 & 16 & 0 & 3.209854 & -0.940339 & 4.316813 \\
\hline 18 & 46 & 0 & 0.942896 & -0.247233 & 4.174997 \\
\hline 19 & 8 & 0 & 0.742093 & -0.476777 & 6.322152 \\
\hline 20 & 6 & 0 & 1.409071 & 0.017956 & 7.216533 \\
\hline 21 & 1 & 0 & 2.272956 & 0.666907 & 7.010842 \\
\hline 22 & 1 & 0 & 1.146302 & -0.190704 & 8.262991 \\
\hline
\end{tabular}

$\mathrm{HF}=-1952.7319479$

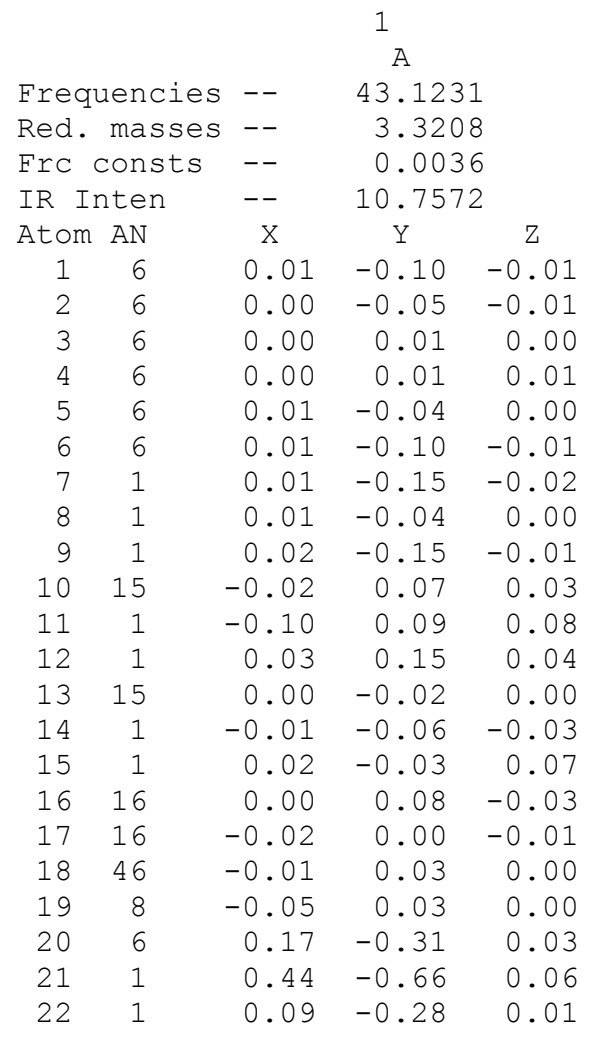

\begin{tabular}{rrr}
\multicolumn{3}{c}{ I A } \\
\multicolumn{3}{c}{53.4126} \\
\multicolumn{3}{c}{4.8420} \\
\multicolumn{3}{c}{0.0081} \\
\multicolumn{3}{c}{ X.5375 } \\
0.03 & \multicolumn{1}{c}{ Y } & \multicolumn{1}{c}{$Z$} \\
0.09 & 0.00 & -0.01 \\
-0.01 & -0.02 & 0.02 \\
0.00 & 0.01 & 0.03 \\
0.02 & 0.10 & 0.04 \\
0.03 & 0.15 & 0.02 \\
0.03 & 0.12 & -0.02 \\
0.03 & 0.14 & 0.06 \\
0.05 & 0.22 & 0.03 \\
-0.01 & -0.05 & 0.04 \\
0.02 & -0.07 & 0.09 \\
-0.02 & -0.05 & 0.03 \\
0.00 & -0.07 & -0.04 \\
-0.02 & -0.09 & -0.04 \\
0.05 & -0.08 & -0.08 \\
-0.05 & -0.10 & -0.02 \\
-0.05 & -0.10 & 0.00 \\
-0.02 & 0.00 & 0.00 \\
0.03 & 0.26 & 0.05 \\
0.29 & 0.11 & -0.06 \\
0.54 & -0.27 & -0.21 \\
0.29 & 0.35 & -0.02
\end{tabular}

\begin{tabular}{rrr}
\multicolumn{3}{c}{ 3 } \\
\multicolumn{3}{c}{ A } \\
\multicolumn{3}{c}{6.8329} \\
\multicolumn{3}{c}{0.1679} \\
\multicolumn{3}{c}{2.0139} \\
X & Y & Z \\
-0.01 & -0.05 & 0.01 \\
0.00 & -0.08 & 0.01 \\
0.01 & -0.01 & 0.00 \\
0.00 & 0.09 & 0.01 \\
-0.01 & 0.13 & 0.02 \\
-0.02 & 0.07 & 0.02 \\
-0.01 & -0.10 & 0.01 \\
-0.02 & 0.22 & 0.02 \\
-0.02 & 0.11 & 0.02 \\
-0.04 & 0.07 & 0.06 \\
-0.15 & 0.09 & 0.25 \\
0.04 & 0.25 & 0.03 \\
0.05 & -0.16 & 0.08 \\
-0.08 & -0.42 & 0.00 \\
0.24 & -0.24 & 0.34 \\
0.07 & 0.22 & -0.05 \\
-0.04 & -0.19 & -0.09 \\
0.00 & -0.01 & -0.01 \\
0.00 & -0.02 & -0.01 \\
-0.10 & 0.13 & -0.02 \\
-0.23 & 0.29 & -0.02 \\
-0.07 & 0.10 & -0.01
\end{tabular}

thermodynamics:

Sum of electronic and zero-point Energies=

$-1952.587641$

Sum of electronic and thermal Enthalpies=

$-1952.571405$

Sum of electronic and thermal Free Energies=

$-1952.631760$

Total free energy in solution:

with all non electrostatic terms

$(\mathrm{a} \cdot \mathrm{u})=$.

Optimized geometry, SCF energy, three lower frequencies, thermochemistry and PCM energy for $\mathbf{T S}_{\mathbf{B 2} \mathbf{B} 3 \text {. }}$ 


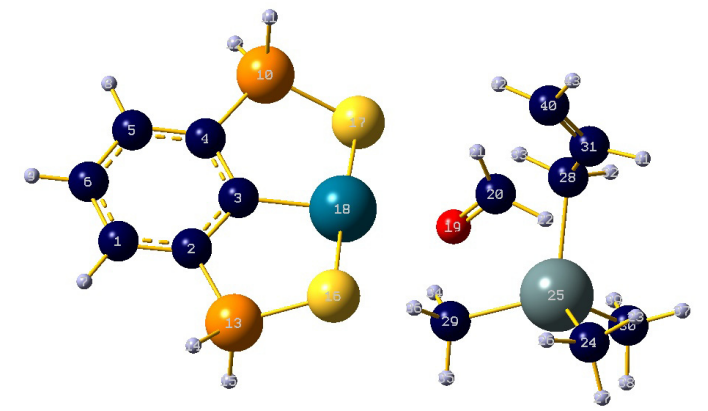

\begin{tabular}{|c|c|c|c|c|c|}
\hline \multirow{2}{*}{$\begin{array}{l}\text { Center } \\
\text { Number }\end{array}$} & \multirow{2}{*}{$\begin{array}{l}\text { Atomic } \\
\text { Number }\end{array}$} & \multirow{2}{*}{$\begin{array}{l}\text { Atomic } \\
\text { Type }\end{array}$} & \multicolumn{3}{|c|}{ Coordinates (Angstroms) } \\
\hline & & & $\mathrm{X}$ & Y & Z \\
\hline 1 & 6 & 0 & -5.170928 & -1.035437 & -0.932946 \\
\hline 2 & 6 & 0 & -3.862957 & -1.100122 & -0.439609 \\
\hline 3 & 6 & 0 & -3.061505 & 0.045593 & -0.228216 \\
\hline 4 & 6 & 0 & -3.687261 & 1.272347 & -0.548544 \\
\hline 5 & 6 & 0 & -4.995440 & 1.358470 & -1.038623 \\
\hline 6 & 6 & 0 & -5.740145 & 0.198908 & -1.233809 \\
\hline 7 & 1 & 0 & -5.753428 & -1.942076 & -1.080362 \\
\hline 8 & 1 & 0 & -5.437584 & 2.323830 & -1.274144 \\
\hline 9 & 1 & 0 & -6.754524 & 0.256430 & -1.615302 \\
\hline 10 & 15 & 0 & -2.660694 & 2.713248 & -0.275916 \\
\hline 11 & 1 & 0 & -3.072396 & 3.350775 & 0.913465 \\
\hline 12 & 1 & 0 & -2.962592 & 3.683935 & -1.251214 \\
\hline 13 & 15 & 0 & -3.066449 & -2.647273 & -0.013952 \\
\hline 14 & 1 & 0 & -4.039191 & -3.531726 & 0.492860 \\
\hline 15 & 1 & 0 & -2.668012 & -3.295645 & -1.201996 \\
\hline 16 & 16 & 0 & -1.561667 & -2.272660 & 1.266901 \\
\hline 17 & 16 & 0 & -0.730739 & 2.140602 & -0.282017 \\
\hline 18 & 46 & 0 & -1.197863 & -0.062362 & 0.473565 \\
\hline 19 & 8 & 0 & 0.794179 & -0.287732 & 1.187956 \\
\hline 20 & 6 & 0 & 1.186172 & 0.021810 & 2.340087 \\
\hline 21 & 1 & 0 & 0.500206 & 0.456285 & 3.076200 \\
\hline 22 & 1 & 0 & 2.082380 & -0.466612 & 2.731703 \\
\hline 23 & 1 & 0 & 4.422554 & -1.553395 & 1.635054 \\
\hline 24 & 6 & 0 & 3.814844 & -1.881103 & 0.785400 \\
\hline 25 & 50 & 0 & 3.636368 & -0.342544 & -0.705231 \\
\hline 26 & 1 & 0 & 2.829577 & -2.191775 & 1.142651 \\
\hline 27 & 1 & 0 & 4.309666 & -2.751274 & 0.341680 \\
\hline 28 & 6 & 0 & 3.390459 & 1.697097 & 0.204945 \\
\hline 29 & 6 & 0 & 2.027955 & -0.686103 & -2.085181 \\
\hline 30 & 6 & 0 & 5.495201 & -0.148863 & -1.769047 \\
\hline 31 & 6 & 0 & 3.261277 & 1.736934 & 1.652908 \\
\hline 32 & 1 & 0 & 4.345963 & 2.135931 & -0.107813 \\
\hline 33 & 1 & 0 & 2.570475 & 2.200732 & -0.314336 \\
\hline 34 & 1 & 0 & 1.920661 & 0.159555 & -2.771689 \\
\hline 35 & 1 & 0 & 2.232775 & -1.583827 & -2.678064 \\
\hline 36 & 1 & 0 & 1.087094 & -0.826978 & -1.546142 \\
\hline 37 & 1 & 0 & 6.320038 & 0.064007 & -1.081144 \\
\hline 38 & 1 & 0 & 5.729399 & -1.076309 & -2.302001 \\
\hline 39 & 1 & 0 & 5.440868 & 0.660775 & -2.504016 \\
\hline 40 & 6 & 0 & 2.140433 & 2.059474 & 2.362960 \\
\hline 41 & 1 & 0 & 4.144634 & 1.440003 & 2.222964 \\
\hline 42 & 1 & 0 & 1.285274 & 2.514611 & 1.869158 \\
\hline 43 & 1 & 0 & 2.193033 & 2.159714 & 3.444361 \\
\hline
\end{tabular}

$\mathrm{HF}=-2193.0855409$

1

Frequencies -- $\quad-69.9095$

Red. masses -- 7.0537

Frc consts -- 0.0203

IR Inten -- 121.3724
2

A

12.7818

6.7819

0.0007

0.1851
3 18.4117 7.1796 0.0014 0.6546 


\begin{tabular}{|c|c|c|c|c|c|c|c|c|c|c|}
\hline Atom & AN & $\mathrm{x}$ & $Y$ & Z & $\mathrm{X}$ & $Y$ & Z & X & Y & Z \\
\hline 1 & 6 & 0.03 & -0.02 & 0.04 & -0.09 & 0.07 & 0.12 & -0.09 & 0.00 & 0.12 \\
\hline 2 & 6 & 0.03 & 0.00 & 0.03 & -0.08 & 0.03 & 0.08 & -0.07 & 0.00 & 0.05 \\
\hline 3 & 6 & 0.03 & 0.00 & 0.02 & -0.02 & 0.00 & 0.02 & -0.07 & 0.01 & 0.03 \\
\hline 4 & 6 & 0.01 & -0.01 & 0.01 & 0.02 & 0.02 & -0.02 & -0.10 & 0.01 & 0.09 \\
\hline 5 & 6 & 0.01 & -0.02 & 0.02 & 0.01 & 0.05 & 0.01 & -0.13 & 0.01 & 0.17 \\
\hline 6 & 6 & 0.02 & -0.02 & 0.03 & -0.04 & 0.08 & 0.08 & -0.13 & 0.00 & 0.19 \\
\hline 7 & 1 & 0.03 & -0.02 & 0.04 & -0.13 & 0.09 & 0.17 & -0.09 & 0.00 & 0.14 \\
\hline 8 & 1 & 0.00 & -0.02 & 0.01 & 0.05 & 0.06 & -0.01 & -0.15 & 0.01 & 0.22 \\
\hline 9 & 1 & 0.02 & -0.03 & 0.03 & -0.05 & 0.11 & 0.10 & -0.15 & 0.00 & 0.25 \\
\hline 10 & 15 & 0.00 & 0.01 & 0.00 & 0.09 & -0.01 & -0.10 & -0.10 & 0.01 & 0.06 \\
\hline 11 & 1 & 0.01 & 0.00 & 0.01 & 0.14 & 0.05 & -0.12 & -0.05 & -0.01 & 0.09 \\
\hline 12 & 1 & -0.03 & 0.01 & 0.01 & 0.11 & -0.04 & -0.14 & -0.15 & 0.03 & 0.10 \\
\hline 13 & 15 & 0.04 & 0.01 & 0.04 & -0.13 & 0.01 & 0.12 & -0.03 & 0.00 & -0.03 \\
\hline 14 & 1 & 0.06 & 0.02 & 0.09 & -0.15 & 0.07 & 0.18 & 0.01 & -0.02 & 0.00 \\
\hline 15 & 1 & -0.01 & -0.02 & 0.03 & -0.19 & -0.05 & 0.14 & -0.08 & 0.04 & -0.07 \\
\hline 16 & 16 & 0.10 & 0.02 & -0.03 & -0.08 & 0.00 & 0.06 & 0.04 & -0.01 & -0.10 \\
\hline 17 & 16 & 0.01 & 0.04 & -0.02 & 0.07 & -0.09 & -0.12 & -0.09 & 0.03 & -0.04 \\
\hline 18 & 46 & 0.06 & 0.03 & -0.01 & -0.01 & -0.05 & -0.03 & -0.03 & 0.02 & -0.05 \\
\hline 19 & 8 & 0.07 & 0.12 & -0.11 & 0.00 & -0.08 & -0.07 & -0.01 & 0.04 & -0.10 \\
\hline 20 & 6 & 0.03 & 0.21 & -0.06 & 0.00 & -0.15 & -0.05 & -0.02 & 0.04 & -0.09 \\
\hline 21 & 1 & -0.14 & -0.06 & -0.06 & -0.01 & -0.23 & -0.01 & -0.03 & 0.03 & -0.10 \\
\hline 22 & 1 & -0.13 & 0.01 & 0.06 & 0.02 & -0.15 & -0.09 & -0.02 & 0.04 & -0.09 \\
\hline 23 & 1 & 0.05 & -0.03 & -0.03 & 0.02 & 0.01 & -0.02 & -0.05 & 0.02 & 0.11 \\
\hline 24 & 6 & -0.03 & -0.01 & 0.03 & 0.07 & 0.01 & -0.06 & 0.01 & 0.00 & 0.07 \\
\hline 25 & 50 & -0.05 & -0.04 & 0.02 & 0.04 & 0.06 & 0.00 & 0.10 & -0.03 & 0.03 \\
\hline 26 & 1 & -0.03 & 0.07 & 0.10 & 0.07 & -0.06 & -0.11 & -0.01 & -0.01 & 0.01 \\
\hline 27 & 1 & -0.12 & -0.04 & 0.00 & 0.14 & 0.05 & -0.07 & 0.05 & 0.00 & 0.12 \\
\hline 28 & 6 & -0.01 & 0.04 & 0.04 & -0.05 & 0.02 & 0.07 & 0.05 & -0.01 & -0.04 \\
\hline 29 & 6 & -0.04 & 0.06 & -0.01 & 0.08 & 0.05 & -0.04 & 0.17 & -0.05 & -0.04 \\
\hline 30 & 6 & -0.04 & 0.07 & 0.07 & 0.05 & 0.16 & 0.04 & 0.17 & -0.07 & 0.14 \\
\hline 31 & 6 & -0.12 & -0.08 & -0.02 & -0.07 & -0.05 & 0.07 & 0.00 & 0.03 & -0.04 \\
\hline 32 & 1 & 0.08 & -0.12 & 0.10 & -0.06 & 0.06 & 0.10 & 0.06 & -0.01 & -0.01 \\
\hline 33 & 1 & 0.06 & 0.05 & -0.05 & -0.06 & 0.01 & 0.08 & 0.07 & -0.03 & -0.08 \\
\hline 34 & 1 & -0.01 & 0.08 & 0.02 & 0.04 & 0.08 & 0.00 & 0.22 & -0.07 & -0.08 \\
\hline 35 & 1 & -0.03 & 0.08 & -0.04 & 0.14 & 0.09 & -0.09 & 0.18 & -0.07 & 0.00 \\
\hline 36 & 1 & -0.03 & 0.05 & -0.02 & 0.08 & -0.04 & -0.07 & 0.14 & -0.01 & -0.09 \\
\hline 37 & 1 & -0.05 & 0.06 & 0.09 & 0.03 & 0.17 & 0.07 & 0.12 & -0.05 & 0.18 \\
\hline 38 & 1 & -0.01 & 0.10 & 0.03 & 0.10 & 0.19 & 0.01 & 0.20 & -0.09 & 0.18 \\
\hline 39 & 1 & -0.05 & 0.10 & 0.10 & 0.04 & 0.19 & 0.07 & 0.22 & -0.09 & 0.11 \\
\hline 40 & 6 & -0.30 & -0.42 & -0.06 & -0.09 & -0.13 & 0.07 & -0.04 & 0.03 & -0.09 \\
\hline 41 & 1 & -0.12 & 0.01 & 0.02 & -0.06 & -0.04 & 0.07 & -0.02 & 0.05 & 0.00 \\
\hline 42 & 1 & -0.22 & -0.31 & -0.08 & -0.10 & -0.14 & 0.08 & -0.02 & 0.02 & -0.13 \\
\hline 43 & 1 & -0.28 & -0.33 & -0.07 & -0.11 & -0.17 & 0.08 & -0.07 & 0.06 & -0.09 \\
\hline
\end{tabular}

thermodynamics:

Sum of electronic and zero-point Energies=

Sum of electronic and thermal Enthalpies=

$-2192.759544$

$-2192.730326$

Sum of electronic and thermal Free Energies=

$-2192.822507$

Total free energy in solution:

with all non electrostatic terms

$(\mathrm{a} \cdot \mathrm{u} \cdot)=-2193.119564$

Optimized geometry, SCF energy, three lower frequencies, thermochemistry and PCM energy for B3. 


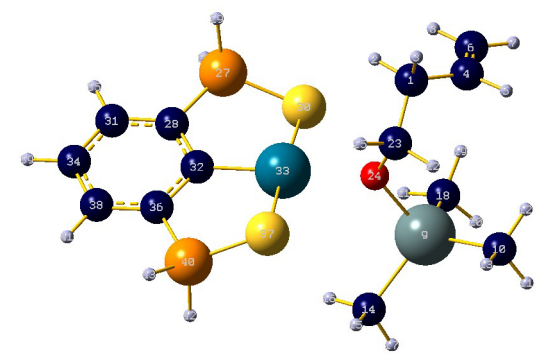

\begin{tabular}{|c|c|c|c|c|c|}
\hline \multirow{2}{*}{$\begin{array}{l}\text { Center } \\
\text { Number }\end{array}$} & \multirow{2}{*}{$\begin{array}{l}\text { Atomic } \\
\text { Number }\end{array}$} & \multirow{2}{*}{$\begin{array}{l}\text { Atomic } \\
\text { Type }\end{array}$} & \multicolumn{3}{|c|}{ Coordinates (Angstroms) } \\
\hline & & & $\mathrm{X}$ & Y & Z \\
\hline 1 & 6 & 0 & -0.019301 & 0.035547 & 0.025423 \\
\hline 2 & 1 & 0 & -0.032764 & 0.029164 & 1.121939 \\
\hline 3 & 1 & 0 & 1.037747 & 0.068613 & -0.281564 \\
\hline 4 & 6 & 0 & -0.718587 & 1.253360 & -0.503149 \\
\hline 5 & 1 & 0 & -0.699700 & 1.377865 & -1.588222 \\
\hline 6 & 6 & 0 & -1.306471 & 2.189374 & 0.244272 \\
\hline 7 & 1 & 0 & -1.764652 & 3.069693 & -0.199214 \\
\hline 8 & 1 & 0 & -1.329978 & 2.119762 & 1.330099 \\
\hline 9 & 50 & 0 & -3.629069 & -1.363993 & -0.935042 \\
\hline 10 & 6 & 0 & -3.062829 & -1.000480 & -2.963137 \\
\hline 11 & 1 & 0 & -3.971419 & -0.931429 & -3.571313 \\
\hline 12 & 1 & 0 & -2.515734 & -0.059527 & -3.065070 \\
\hline 13 & 1 & 0 & -2.452676 & -1.816762 & -3.361108 \\
\hline 14 & 6 & 0 & -4.611509 & -3.240345 & -0.644438 \\
\hline 15 & 1 & 0 & -4.004941 & -4.057773 & -1.043471 \\
\hline 16 & 1 & 0 & -4.793754 & -3.416454 & 0.420215 \\
\hline 17 & 1 & 0 & -5.577639 & -3.233812 & -1.160857 \\
\hline 18 & 6 & 0 & -4.629906 & 0.251158 & 0.035750 \\
\hline 19 & 1 & 0 & -3.958611 & 1.109482 & 0.117159 \\
\hline 20 & 1 & 0 & -5.508872 & 0.542820 & -0.549177 \\
\hline 21 & 1 & 0 & -4.961901 & -0.042895 & 1.035568 \\
\hline 22 & 1 & 0 & -0.666358 & -1.256215 & -1.588020 \\
\hline 23 & 6 & 0 & -0.579794 & -1.287204 & -0.493709 \\
\hline 24 & 8 & 0 & -1.861472 & -1.617367 & 0.064218 \\
\hline 25 & 1 & 0 & 0.111117 & -2.102606 & -0.248190 \\
\hline 26 & 1 & 0 & -2.571641 & -0.859088 & 5.915829 \\
\hline 27 & 15 & 0 & -1.799936 & -1.341959 & 4.840189 \\
\hline 28 & 6 & 0 & -1.807258 & -3.131698 & 4.881485 \\
\hline 29 & 1 & 0 & -0.508081 & -0.887632 & 5.179925 \\
\hline 30 & 16 & 0 & -2.438500 & -0.719873 & 3.035590 \\
\hline 31 & 6 & 0 & -1.797089 & -3.850507 & 6.082524 \\
\hline 32 & 6 & 0 & -1.817259 & -3.750386 & 3.609300 \\
\hline 33 & 46 & 0 & -1.830477 & -2.713840 & 1.902271 \\
\hline 34 & 6 & 0 & -1.799853 & -5.242006 & 6.059726 \\
\hline 35 & 1 & 0 & -1.790371 & -3.332360 & 7.038892 \\
\hline 36 & 6 & 0 & -1.817457 & -5.164794 & 3.644215 \\
\hline 37 & 16 & 0 & -1.189690 & -4.634396 & 0.657366 \\
\hline 38 & 6 & 0 & -1.810774 & -5.901547 & 4.834226 \\
\hline 39 & 1 & 0 & -1.793086 & -5.806150 & 6.986808 \\
\hline 40 & 15 & 0 & -1.832159 & -5.950428 & 2.034990 \\
\hline 41 & 1 & 0 & -1.809746 & -6.989062 & 4.813288 \\
\hline 42 & 1 & 0 & -3.126737 & -6.456488 & 1.791289 \\
\hline 43 & 1 & 0 & -1.063678 & -7.129908 & 2.096866 \\
\hline
\end{tabular}

$\mathrm{HF}=-2193.1466588$

\begin{tabular}{|c|c|c|c|c|c|c|c|c|c|}
\hline \multirow[b]{3}{*}{ Frequencies } & \multicolumn{3}{|c|}{1} & \multicolumn{3}{|c|}{2} & \multicolumn{3}{|c|}{3} \\
\hline & & A & & & A & & & A & \\
\hline & -- & 20.3084 & & & $24.28 \varepsilon$ & & & 27.2836 & \\
\hline Red. masses & -- & 4.2167 & & & 4.976 & & & 4.0548 & \\
\hline Frc consts & -- & 0.0010 & & & 0.001 & & & 0.0018 & \\
\hline IR Inten & -- & 0.0227 & & & $0.27^{\circ}$ & & & 0.6856 & \\
\hline Atom AN & $\mathrm{x}$ & Y & Z & X & Y & Z & X & $Y$ & Z \\
\hline 6 & 0.04 & -0.09 & 0.05 & -0.03 & 0.03 & 0.09 & 0.02 & -0.08 & 0.0 \\
\hline
\end{tabular}




\begin{tabular}{|c|c|c|c|c|c|c|c|c|c|c|}
\hline 2 & 1 & 0.11 & -0.18 & 0.05 & -0.02 & -0.03 & 0.09 & 0.00 & -0.08 & -0.01 \\
\hline 3 & 1 & 0.03 & -0.10 & -0.01 & -0.04 & 0.07 & 0.08 & 0.03 & -0.10 & 0.02 \\
\hline 4 & 6 & 0.05 & -0.02 & 0.19 & -0.07 & 0.04 & 0.16 & 0.05 & -0.07 & -0.02 \\
\hline 5 & 1 & -0.04 & 0.05 & 0.20 & -0.08 & 0.10 & 0.17 & 0.11 & -0.06 & -0.02 \\
\hline 6 & 6 & 0.16 & -0.05 & 0.31 & -0.09 & -0.02 & 0.21 & 0.01 & -0.08 & -0.04 \\
\hline 7 & 1 & 0.17 & 0.01 & 0.41 & -0.12 & -0.01 & 0.27 & 0.04 & -0.07 & -0.06 \\
\hline 8 & 1 & 0.25 & -0.13 & 0.31 & -0.08 & -0.08 & 0.21 & -0.04 & -0.09 & -0.04 \\
\hline 9 & 50 & -0.03 & 0.00 & -0.02 & 0.01 & -0.04 & -0.05 & 0.00 & 0.04 & -0.01 \\
\hline 10 & 6 & -0.02 & -0.08 & -0.03 & 0.06 & 0.07 & -0.02 & -0.01 & 0.26 & 0.03 \\
\hline 11 & 1 & -0.01 & -0.08 & -0.04 & 0.07 & 0.07 & -0.04 & -0.02 & 0.33 & 0.05 \\
\hline 12 & 1 & 0.01 & -0.10 & -0.06 & 0.03 & 0.10 & 0.04 & -0.01 & 0.27 & 0.13 \\
\hline 13 & 1 & -0.04 & -0.11 & 0.00 & 0.09 & 0.11 & -0.04 & -0.02 & 0.30 & -0.07 \\
\hline 14 & 6 & -0.08 & 0.04 & 0.06 & 0.06 & -0.08 & -0.16 & 0.02 & 0.00 & -0.21 \\
\hline 15 & 1 & -0.11 & 0.01 & 0.06 & 0.11 & -0.05 & -0.15 & 0.06 & 0.04 & -0.23 \\
\hline 16 & 1 & -0.05 & 0.08 & 0.07 & 0.01 & -0.12 & -0.18 & -0.03 & -0.09 & -0.23 \\
\hline 17 & 1 & -0.09 & 0.06 & 0.09 & 0.09 & -0.10 & -0.21 & 0.05 & 0.02 & -0.26 \\
\hline 18 & 6 & 0.04 & 0.08 & -0.08 & -0.07 & -0.11 & 0.00 & -0.02 & -0.06 & 0.15 \\
\hline 19 & 1 & 0.09 & 0.04 & -0.07 & -0.10 & -0.08 & 0.03 & -0.03 & -0.06 & 0.22 \\
\hline 20 & 1 & 0.08 & 0.12 & -0.12 & -0.07 & -0.13 & 0.00 & -0.03 & -0.03 & 0.19 \\
\hline 21 & 1 & -0.01 & 0.11 & -0.09 & -0.06 & -0.15 & -0.01 & 0.00 & -0.15 & 0.13 \\
\hline 22 & 1 & -0.06 & 0.06 & -0.03 & 0.01 & 0.08 & 0.03 & 0.01 & -0.07 & -0.01 \\
\hline 23 & 6 & -0.03 & -0.03 & -0.03 & -0.01 & 0.04 & 0.03 & 0.00 & -0.07 & -0.01 \\
\hline 24 & 8 & -0.02 & -0.02 & -0.03 & -0.01 & 0.00 & 0.00 & -0.01 & -0.05 & -0.02 \\
\hline 25 & 1 & -0.05 & -0.08 & -0.12 & 0.00 & 0.04 & 0.00 & -0.01 & -0.08 & -0.01 \\
\hline 26 & 1 & -0.10 & 0.01 & -0.04 & 0.26 & 0.07 & 0.04 & -0.08 & 0.02 & -0.04 \\
\hline 27 & 15 & -0.06 & 0.03 & -0.02 & 0.15 & 0.02 & -0.02 & -0.05 & 0.02 & -0.02 \\
\hline 28 & 6 & 0.00 & 0.03 & -0.01 & 0.09 & 0.02 & 0.00 & 0.00 & 0.02 & 0.00 \\
\hline 29 & 1 & -0.08 & 0.07 & 0.01 & 0.20 & -0.02 & -0.14 & -0.07 & 0.06 & -0.01 \\
\hline 30 & 16 & -0.03 & 0.00 & -0.04 & 0.02 & 0.01 & 0.02 & -0.05 & -0.02 & -0.03 \\
\hline 31 & 6 & 0.03 & 0.04 & -0.01 & 0.11 & 0.03 & 0.01 & 0.03 & 0.05 & 0.02 \\
\hline 32 & 6 & 0.01 & 0.01 & -0.01 & 0.01 & 0.02 & 0.01 & 0.01 & 0.00 & 0.01 \\
\hline 33 & 46 & -0.01 & 0.00 & -0.02 & -0.01 & 0.01 & 0.00 & -0.02 & -0.03 & 0.00 \\
\hline 34 & 6 & 0.07 & 0.04 & 0.01 & 0.04 & 0.03 & 0.01 & 0.08 & 0.05 & 0.04 \\
\hline 35 & 1 & 0.01 & 0.05 & -0.01 & 0.17 & 0.03 & 0.00 & 0.03 & 0.06 & 0.01 \\
\hline 36 & 6 & 0.06 & 0.01 & 0.01 & -0.05 & 0.02 & 0.01 & 0.05 & 0.00 & 0.04 \\
\hline 37 & 16 & -0.01 & 0.00 & -0.01 & -0.05 & -0.01 & 0.01 & -0.02 & -0.04 & 0.02 \\
\hline 38 & 6 & 0.09 & 0.03 & 0.02 & -0.04 & 0.02 & 0.01 & 0.09 & 0.02 & 0.05 \\
\hline 39 & 1 & 0.10 & 0.05 & 0.02 & 0.06 & 0.03 & 0.01 & 0.11 & 0.06 & 0.05 \\
\hline 40 & 15 & 0.07 & -0.01 & 0.02 & -0.14 & 0.03 & 0.01 & 0.06 & -0.03 & 0.06 \\
\hline 41 & 1 & 0.13 & 0.03 & 0.03 & -0.09 & 0.02 & 0.02 & 0.12 & 0.02 & 0.07 \\
\hline 42 & 1 & 0.09 & -0.07 & 0.05 & -0.18 & 0.13 & 0.02 & 0.07 & -0.09 & 0.09 \\
\hline 43 & 1 & 0.12 & 0.02 & 0.03 & -0.23 & -0.03 & -0.01 & 0.11 & 0.00 & 0.07 \\
\hline
\end{tabular}

thermodynamics:

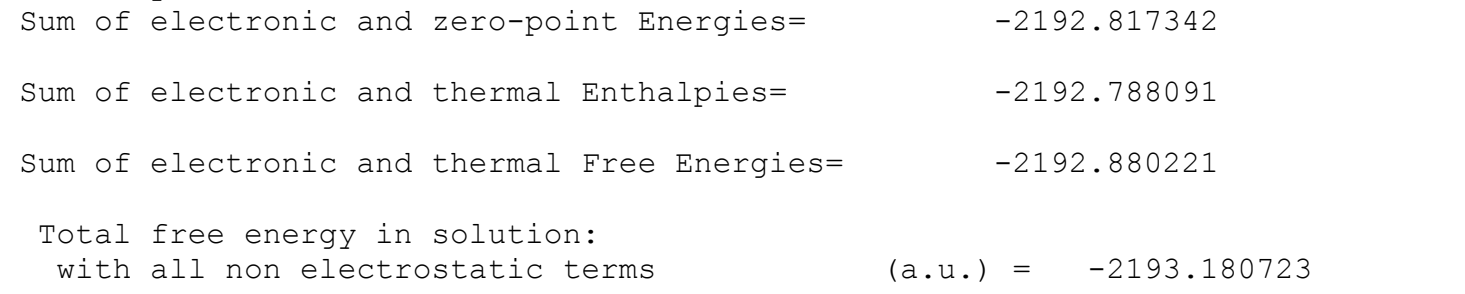

Optimized geometry, SCF energy, three lower frequencies, thermochemistry and PCM energy for $\mathbf{T S}_{\mathbf{B} 3 \mathbf{B} 2}$. 


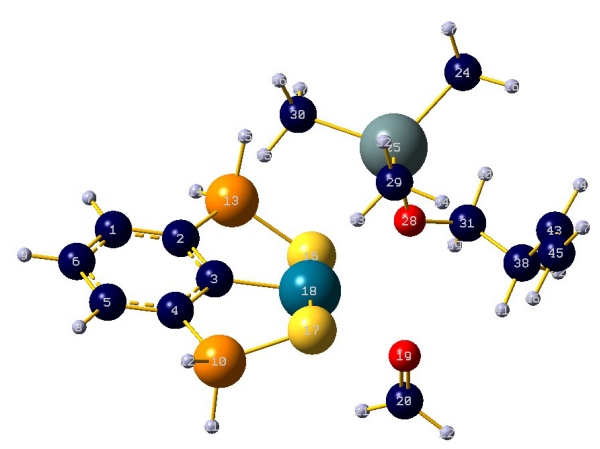

\begin{tabular}{|c|c|c|c|c|c|}
\hline \multirow{2}{*}{$\begin{array}{l}\text { Center } \\
\text { Number }\end{array}$} & \multirow{2}{*}{$\begin{array}{l}\text { Atomic } \\
\text { Number }\end{array}$} & \multirow{2}{*}{$\begin{array}{l}\text { Atomic } \\
\text { Type }\end{array}$} & \multicolumn{3}{|c|}{ Coordinates (Angstroms) } \\
\hline & & & $\mathrm{x}$ & Y & Z \\
\hline 1 & 6 & 0 & 4.337136 & -1.642512 & -0.785777 \\
\hline 2 & 6 & 0 & 3.184623 & -0.865043 & -0.941902 \\
\hline 3 & 6 & 0 & 2.548972 & -0.210581 & 0.139556 \\
\hline 4 & 6 & 0 & 3.169655 & -0.391193 & 1.398556 \\
\hline 5 & 6 & 0 & 4.324454 & -1.163238 & 1.568875 \\
\hline 6 & 6 & 0 & 4.909661 & -1.793524 & 0.474357 \\
\hline 7 & 1 & 0 & 4.796355 & -2.127754 & -1.643912 \\
\hline 8 & 1 & 0 & 4.769963 & -1.279469 & 2.554195 \\
\hline 9 & 1 & 0 & 5.804641 & -2.393939 & 0.601485 \\
\hline 10 & 15 & 0 & 2.344887 & 0.451886 & 2.743282 \\
\hline 11 & 1 & 0 & 3.057618 & 1.632149 & 3.044511 \\
\hline 12 & 1 & 0 & 2.510038 & -0.301785 & 3.922408 \\
\hline 13 & 15 & 0 & 2.390419 & -0.588114 & -2.520478 \\
\hline 14 & 1 & 0 & 3.367381 & -0.629250 & -3.534918 \\
\hline 15 & 1 & 0 & 1.583135 & -1.702154 & -2.828813 \\
\hline 16 & 16 & 0 & 1.389287 & 1.151846 & -2.419385 \\
\hline 17 & 16 & 0 & 0.424943 & 0.742438 & 2.214417 \\
\hline 18 & 46 & 0 & 0.920732 & 0.886059 & -0.098170 \\
\hline 19 & 8 & 0 & -0.198238 & 3.031798 & -0.193677 \\
\hline 20 & 6 & 0 & 0.365043 & 4.078149 & -0.446749 \\
\hline 21 & 1 & 0 & 1.412288 & 4.110594 & -0.792693 \\
\hline 22 & 1 & 0 & -0.164708 & 5.038895 & -0.339074 \\
\hline 23 & 1 & 0 & -3.629579 & -1.810974 & -2.334453 \\
\hline 24 & 6 & 0 & -3.875198 & -2.003093 & -1.285403 \\
\hline 25 & 50 & 0 & -2.231329 & -1.518433 & 0.000480 \\
\hline 26 & 1 & 0 & -4.773864 & -1.440300 & -1.022424 \\
\hline 27 & 1 & 0 & -4.096488 & -3.071042 & -1.181065 \\
\hline 28 & 8 & 0 & -1.616097 & 0.336818 & -0.440021 \\
\hline 29 & 6 & 0 & -2.664259 & -1.667732 & 2.088887 \\
\hline 30 & 6 & 0 & -0.533420 & -2.729939 & -0.520685 \\
\hline 31 & 6 & 0 & -2.397545 & 1.190153 & -1.266624 \\
\hline 32 & 1 & 0 & -2.970371 & -2.692924 & 2.324364 \\
\hline 33 & 1 & 0 & -1.782423 & -1.421610 & 2.686579 \\
\hline 34 & 1 & 0 & -3.477207 & -0.988190 & 2.354953 \\
\hline 35 & 1 & 0 & 0.383421 & -2.320269 & -0.085389 \\
\hline 36 & 1 & 0 & -0.664105 & -3.751159 & -0.146464 \\
\hline 37 & 1 & 0 & -0.428273 & -2.780692 & -1.609797 \\
\hline 38 & 6 & 0 & -3.331936 & 2.113674 & -0.477325 \\
\hline 39 & 1 & 0 & -1.703909 & 1.804514 & -1.853250 \\
\hline 40 & 1 & 0 & -2.985600 & 0.604801 & -1.991020 \\
\hline 41 & 1 & 0 & -2.748903 & 2.616251 & 0.301815 \\
\hline 42 & 1 & 0 & -3.680704 & 2.891192 & -1.175360 \\
\hline 43 & 6 & 0 & -4.527474 & 1.430973 & 0.116610 \\
\hline 44 & 1 & 0 & -5.223103 & 0.983252 & -0.596429 \\
\hline 45 & 6 & 0 & -4.811361 & 1.366540 & 1.419462 \\
\hline 46 & 1 & 0 & -4.162623 & 1.820857 & 2.166372 \\
\hline 47 & 1 & 0 & -5.712880 & 0.882080 & 1.786232 \\
\hline
\end{tabular}

$\mathrm{HF}=-2307.5841398$ 


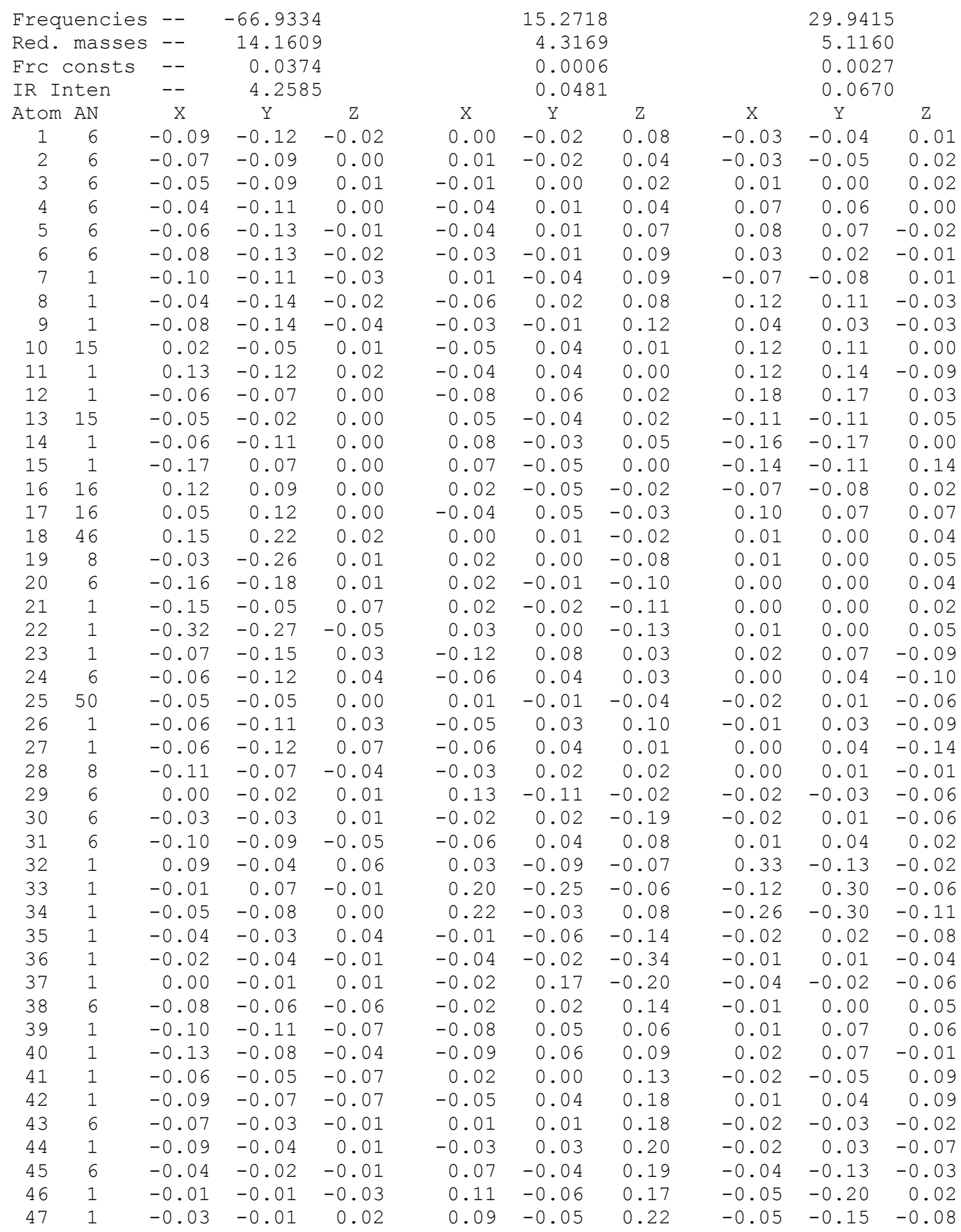

thermodynamics:

Sum of electronic and zero-point Energies=

$-2307.226683$

Sum of electronic and thermal Enthalpies=

$-2307.193827$

Sum of electronic and thermal Free Energies=

$-2307.294295$

Total free energy in solution:

with all non electrostatic terms

$(\mathrm{a} \cdot \mathrm{u})=$.

IV. Comparison with literature complexes 


\section{Se $\sim \mathrm{C} \sim \mathrm{Se}$ complex}

Optimized geometry, SCF energy, three lower frequencies, thermochemistry and PCM energy for B2.

\begin{tabular}{|c|c|c|c|c|c|}
\hline $\begin{array}{l}\text { Center } \\
\text { Number }\end{array}$ & $\begin{array}{l}\text { Atomic } \\
\text { Number }\end{array}$ & $\begin{array}{c}\text { Atomic } \\
\text { Type }\end{array}$ & \multicolumn{3}{|c|}{ Coordinates (Angstroms) } \\
\hline 1 & 6 & 0 & 0.016329 & 0.052567 & 0.029902 \\
\hline 2 & 6 & 0 & 0.035164 & 0.064217 & 1.429538 \\
\hline 3 & 6 & 0 & 1.263832 & -0.016458 & 2.114499 \\
\hline 4 & 6 & 0 & 2.460972 & -0.089930 & 1.375335 \\
\hline 5 & 6 & 0 & 2.418994 & -0.054579 & -0.023360 \\
\hline 6 & 6 & 0 & 1.202420 & 0.005772 & -0.694067 \\
\hline 7 & 1 & 0 & -0.936266 & 0.088525 & -0.493037 \\
\hline 8 & 1 & 0 & 3.347739 & -0.083473 & -0.587960 \\
\hline 9 & 1 & 0 & 1.178807 & 0.015479 & -1.779430 \\
\hline 10 & 46 & 0 & 1.303322 & -0.004389 & 4.101478 \\
\hline 11 & 8 & 0 & 1.319756 & -0.022582 & 6.327128 \\
\hline 12 & 6 & 0 & 1.340018 & 0.882953 & 7.143807 \\
\hline 13 & 1 & 0 & 1.371119 & 1.941899 & 6.842199 \\
\hline 14 & 1 & 0 & 1.328298 & 0.650374 & 8.218733 \\
\hline 15 & 6 & 0 & -1.257424 & 0.185563 & 2.171226 \\
\hline 16 & 6 & 0 & 3.783975 & -0.235075 & 2.055215 \\
\hline 17 & 1 & 0 & -2.106441 & -0.246912 & 1.638229 \\
\hline 18 & 1 & 0 & -1.490227 & 1.216848 & 2.451250 \\
\hline 19 & 1 & 0 & 4.612605 & 0.202314 & 1.494964 \\
\hline 20 & 1 & 0 & 4.020193 & -1.273274 & 2.305356 \\
\hline 21 & 34 & 0 & 3.679232 & 0.722909 & 3.834263 \\
\hline 22 & 34 & 0 & -1.053386 & -0.812717 & 3.916457 \\
\hline 23 & 1 & 0 & 4.349565 & -0.344088 & 4.639161 \\
\hline 24 & 1 & 0 & -1.756867 & 0.193605 & 4.770804 \\
\hline
\end{tabular}

$\mathrm{HF}=-569.6996904$

\begin{tabular}{|c|c|c|c|c|}
\hline \multicolumn{5}{|c|}{1} \\
\hline \multicolumn{2}{|c|}{ Frequencies } & -- & 38.89 & \\
\hline Red. & masses & -- & 2.59 & \\
\hline Frc $C$ & onsts & -- & 0.00 & \\
\hline IR In & iten & -- & 10.80 & \\
\hline Atom & AN & $\mathrm{X}$ & Y & $\mathrm{Z}$ \\
\hline 1 & 6 & 0.01 & -0.01 & -0.01 \\
\hline 2 & 6 & 0.00 & -0.02 & -0.01 \\
\hline 3 & 6 & 0.00 & 0.00 & 0.00 \\
\hline 4 & 6 & 0.00 & 0.01 & 0.01 \\
\hline 5 & 6 & 0.02 & 0.01 & 0.01 \\
\hline 6 & 6 & 0.02 & 0.00 & 0.00 \\
\hline 7 & 1 & 0.02 & -0.02 & -0.02 \\
\hline 8 & 1 & 0.02 & 0.02 & 0.02 \\
\hline 9 & 1 & 0.03 & 0.00 & 0.00 \\
\hline 10 & 46 & -0.02 & 0.00 & 0.00 \\
\hline 11 & 8 & -0.09 & 0.02 & 0.00 \\
\hline 12 & 6 & 0.34 & 0.02 & -0.01 \\
\hline 13 & 1 & 0.91 & 0.00 & -0.02 \\
\hline 14 & 1 & 0.16 & 0.04 & -0.01 \\
\hline 15 & 6 & -0.01 & -0.03 & -0.02 \\
\hline 16 & 6 & 0.00 & 0.02 & 0.02 \\
\hline 17 & 1 & 0.00 & -0.04 & -0.03 \\
\hline
\end{tabular}

\begin{tabular}{|c|c|c|c|c|}
\hline \multicolumn{2}{|l|}{2} & \multicolumn{3}{|c|}{3} \\
\hline \multicolumn{2}{|l|}{ A } & \multicolumn{3}{|c|}{ A } \\
\hline \multicolumn{2}{|c|}{45.0349} & & \multicolumn{2}{|c|}{62.2374} \\
\hline \multicolumn{2}{|c|}{6.4322} & & \multicolumn{2}{|c|}{4.2987} \\
\hline \multicolumn{2}{|c|}{0.0077} & & \multicolumn{2}{|c|}{0.0098} \\
\hline \multicolumn{2}{|c|}{1.9207} & & \multicolumn{2}{|c|}{0.4922} \\
\hline Y & Z & $\mathrm{X}$ & Y & Z \\
\hline 0.11 & 0.01 & 0.01 & 0.15 & 0.04 \\
\hline 0.03 & 0.02 & 0.01 & 0.12 & 0.04 \\
\hline 0.01 & 0.00 & 0.00 & -0.01 & 0.03 \\
\hline 0.06 & -0.01 & -0.01 & -0.13 & 0.04 \\
\hline 0.14 & -0.01 & 0.00 & -0.12 & 0.04 \\
\hline 0.17 & 0.00 & 0.00 & 0.03 & 0.04 \\
\hline 0.13 & 0.02 & 0.01 & 0.26 & 0.04 \\
\hline 0.18 & -0.02 & 0.00 & -0.21 & 0.04 \\
\hline 0.24 & 0.00 & 0.01 & 0.04 & 0.04 \\
\hline 0.03 & 0.00 & 0.00 & 0.00 & 0.03 \\
\hline 0.23 & 0.00 & -0.02 & 0.03 & 0.03 \\
\hline 0.35 & -0.13 & 0.03 & 0.04 & 0.02 \\
\hline 0.30 & -0.29 & 0.10 & 0.03 & 0.00 \\
\hline 0.51 & -0.10 & -0.01 & 0.05 & 0.02 \\
\hline-0.05 & 0.03 & 0.03 & 0.19 & 0.08 \\
\hline 0.00 & -0.04 & -0.04 & -0.23 & 0.08 \\
\hline-0.06 & 0.02 & -0.03 & 0.35 & 0.05 \\
\hline
\end{tabular}




\begin{tabular}{|c|c|c|c|c|c|c|c|c|c|}
\hline 18 & -0.02 & -0.03 & -0.04 & -0.06 & -0.07 & 0.07 & 0.16 & 0.18 & 0.22 \\
\hline 19 & 0.00 & 0.03 & 0.02 & -0.02 & 0.03 & -0.02 & 0.03 & -0.42 & 0.05 \\
\hline 1 & 0.01 & 0.02 & 0.01 & -0.02 & -0.02 & -0.11 & -0.20 & -0.22 & 0.25 \\
\hline 34 & -0.02 & 0.00 & 0.02 & 0.04 & -0.12 & 0.03 & -0.02 & 0.05 & -0.06 \\
\hline 34 & -0.01 & -0.01 & -0.02 & 0.04 & -0.10 & -0.01 & 0.02 & -0.07 & -0.06 \\
\hline 1 & -0.02 & -0.01 & 0.02 & -0.07 & -0.23 & -0.03 & 0.05 & 0.21 & 0.09 \\
\hline 1 & -0.02 & -0.01 & -0.03 & -0.04 & -0.18 & 0.03 & -0.04 & -0.22 & 0.08 \\
\hline
\end{tabular}

thermodynamics:

Sum of electronic and zero-point Energies=

$-569.525806$

Sum of electronic and thermal Enthalpies=

$-569.509423$

Sum of electronic and thermal Free Energies=

$-569.570916$

Total free energy in solution:

with all non electrostatic terms

$(\mathrm{a} \cdot \mathrm{u} \cdot)=-569.738641$

Optimized geometry, SCF energy, three lower frequencies, thermochemistry and PCM energy for $\mathbf{T S}_{\mathbf{B 2} \mathbf{B} 3}$.

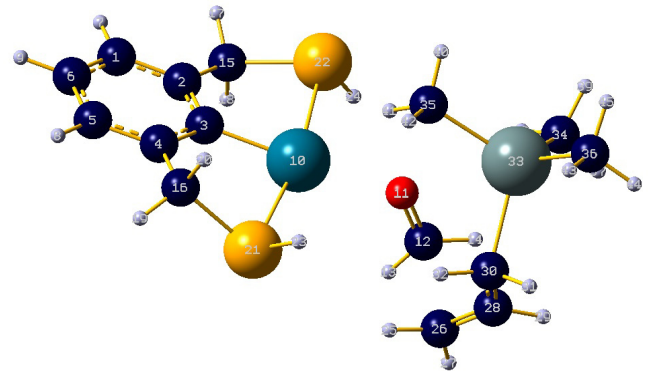

\begin{tabular}{|c|c|c|c|c|c|}
\hline \multirow{2}{*}{$\begin{array}{l}\text { Center } \\
\text { Number }\end{array}$} & \multirow{2}{*}{$\begin{array}{l}\text { Atomic } \\
\text { Number }\end{array}$} & \multirow{2}{*}{$\begin{array}{l}\text { Atomic } \\
\text { Type }\end{array}$} & \multicolumn{3}{|c|}{ Coordinates (Angstroms) } \\
\hline & & & $\mathrm{x}$ & Y & Z \\
\hline 1 & 6 & 0 & -0.156536 & 0.119623 & -0.232310 \\
\hline 2 & 6 & 0 & -0.044723 & 0.127770 & 1.162750 \\
\hline 3 & 6 & 0 & 1.226523 & 0.097851 & 1.773270 \\
\hline 4 & 6 & 0 & 2.371045 & 0.083764 & 0.949922 \\
\hline 5 & 6 & 0 & 2.239019 & 0.121073 & -0.442968 \\
\hline 6 & 6 & 0 & 0.980066 & 0.127474 & -1.033437 \\
\hline 7 & 1 & 0 & -1.142098 & 0.117943 & -0.691945 \\
\hline 8 & 1 & 0 & 3.129408 & 0.134686 & -1.067134 \\
\hline 9 & 1 & 0 & 0.884966 & 0.138909 & -2.114986 \\
\hline 10 & 46 & 0 & 1.394568 & 0.093295 & 3.760644 \\
\hline 11 & 8 & 0 & 1.459368 & 0.027068 & 5.928471 \\
\hline 12 & 6 & 0 & 1.381704 & 1.035358 & 6.685447 \\
\hline 13 & 1 & 0 & 1.311081 & 2.046188 & 6.262805 \\
\hline 14 & 1 & 0 & 0.936504 & 0.907072 & 7.678024 \\
\hline 15 & 6 & 0 & -1.293181 & 0.199668 & 1.984385 \\
\hline 16 & 6 & 0 & 3.742575 & -0.002077 & 1.539095 \\
\hline 17 & 1 & 0 & -2.154660 & -0.273219 & 1.508497 \\
\hline 18 & 1 & 0 & -1.552998 & 1.223712 & 2.268039 \\
\hline 19 & 1 & 0 & 4.509196 & 0.487303 & 0.934977 \\
\hline 20 & 1 & 0 & 4.048186 & -1.030576 & 1.752155 \\
\hline 21 & 34 & 0 & 3.705551 & 0.915502 & 3.341653 \\
\hline 22 & 34 & 0 & -0.942640 & -0.763552 & 3.724616 \\
\hline 23 & 1 & 0 & 4.474062 & -0.135563 & 4.075466 \\
\hline 24 & 1 & 0 & -1.595587 & 0.245584 & 4.613636 \\
\hline 25 & 1 & 0 & 3.776977 & 1.701233 & 6.465569 \\
\hline 26 & 6 & 0 & 3.299041 & 1.622159 & 7.439726 \\
\hline 27 & 1 & 0 & 2.971046 & 2.558343 & 7.884543 \\
\hline 28 & 6 & 0 & 3.570824 & 0.549738 & 8.252268 \\
\hline 29 & 1 & 0 & 3.231119 & 0.616115 & 9.288196 \\
\hline 30 & 6 & 0 & 4.196458 & -0.693722 & 7.866400 \\
\hline 31 & 1 & 0 & 4.846460 & -1.074407 & 8.662298 \\
\hline 32 & 1 & 0 & 4.752052 & -0.635242 & 6.926436 \\
\hline
\end{tabular}




$\begin{array}{rr}33 & 50 \\ 34 & 6 \\ 35 & 6 \\ 36 & 6 \\ 37 & 1 \\ 38 & 1 \\ 39 & 1 \\ 40 & 1 \\ 41 & 1 \\ 42 & 1 \\ 43 & 1 \\ 44 & 1 \\ 45 & 1\end{array}$

$\begin{array}{lll}2.824642 & -2.487305 & 7.701883 \\ 0.875867 & -2.099167 & 8.512957 \\ 2.825692 & -3.134232 & 5.654944 \\ 3.862180 & -3.917111 & 8.924608 \\ 0.938960 & -1.585676 & 9.477399 \\ 0.284568 & -1.497339 & 7.819103 \\ 0.360425 & -3.051359 & 8.675930 \\ 2.271931 & -4.074321 & 5.560711 \\ 2.352465 & -2.384277 & 5.014218 \\ 3.850174 & -3.311434 & 5.311523 \\ 4.881753 & -4.076660 & 8.559335 \\ 3.914957 & -3.578710 & 9.964417 \\ 3.340571 & -4.879719 & 8.907559\end{array}$

$\mathrm{HF}=-810.0525161$

\begin{tabular}{|c|c|c|c|c|c|c|c|}
\hline & \multicolumn{3}{|c|}{1} & \multicolumn{3}{|c|}{2} \\
\hline & & \multirow{2}{*}{\multicolumn{3}{|c|}{$\begin{array}{c}A \\
159.0793\end{array}$}} & \multicolumn{3}{|c|}{ A } \\
\hline \multirow{2}{*}{\multicolumn{2}{|c|}{$\begin{array}{l}\text { Frequencies } \\
\text { Red masses }\end{array}$}} & & & & \multicolumn{3}{|c|}{8.7785} \\
\hline & & $s--$ & \multicolumn{2}{|c|}{7.5628} & \multicolumn{3}{|c|}{7.5939} \\
\hline \multicolumn{2}{|c|}{ Frc consts } & -- & \multicolumn{2}{|c|}{0.1128} & \multicolumn{3}{|c|}{0.0003} \\
\hline \multicolumn{2}{|c|}{ IR Inten } & -- & \multicolumn{2}{|c|}{271.8348} & \multicolumn{3}{|c|}{0.1912} \\
\hline Atom & AN & $\mathrm{X}$ & Y & Z & $\mathrm{x}$ & $\mathrm{Y}$ & Z \\
\hline 1 & 6 & 0.00 & 0.00 & -0.01 & -0.03 & -0.11 & 0.02 \\
\hline 2 & 6 & 0.00 & 0.00 & -0.01 & -0.01 & -0.08 & 0.02 \\
\hline 3 & 6 & 0.00 & 0.00 & -0.02 & 0.00 & -0.01 & 0.00 \\
\hline 4 & 6 & 0.01 & 0.00 & -0.01 & -0.01 & 0.04 & -0.02 \\
\hline 5 & 6 & 0.01 & 0.00 & -0.01 & -0.03 & 0.01 & -0.02 \\
\hline 6 & 6 & 0.01 & 0.00 & -0.01 & -0.04 & -0.07 & 0.00 \\
\hline 7 & 1 & 0.01 & 0.00 & -0.01 & -0.04 & -0.17 & 0.04 \\
\hline 8 & 1 & 0.01 & 0.00 & -0.01 & -0.05 & 0.04 & -0.03 \\
\hline 9 & 1 & 0.01 & 0.00 & -0.01 & -0.06 & -0.09 & 0.00 \\
\hline 10 & 46 & -0.01 & 0.00 & -0.03 & 0.04 & 0.03 & 0.00 \\
\hline 11 & 8 & -0.17 & 0.04 & 0.00 & 0.05 & 0.05 & 0.00 \\
\hline 12 & 6 & -0.32 & -0.08 & -0.03 & 0.11 & 0.06 & 0.00 \\
\hline 13 & 1 & 0.06 & 0.01 & 0.12 & 0.16 & 0.06 & 0.00 \\
\hline 14 & 1 & 0.04 & -0.13 & 0.11 & 0.12 & 0.08 & 0.00 \\
\hline 15 & 6 & 0.00 & 0.00 & -0.01 & 0.00 & -0.13 & 0.04 \\
\hline 16 & 6 & 0.00 & 0.00 & 0.00 & 0.00 & 0.12 & -0.04 \\
\hline 17 & 1 & 0.00 & 0.01 & -0.02 & 0.02 & -0.18 & 0.06 \\
\hline 18 & 1 & 0.00 & 0.00 & -0.01 & -0.05 & -0.14 & 0.02 \\
\hline 19 & 1 & 0.01 & -0.01 & 0.01 & -0.03 & 0.15 & -0.06 \\
\hline 20 & 1 & 0.00 & -0.01 & 0.00 & 0.06 & 0.14 & -0.03 \\
\hline 21 & 34 & 0.00 & 0.00 & 0.00 & -0.01 & 0.15 & -0.06 \\
\hline 22 & 34 & -0.01 & -0.01 & -0.02 & 0.08 & -0.08 & 0.05 \\
\hline 23 & 1 & -0.02 & 0.00 & 0.02 & 0.06 & 0.20 & -0.05 \\
\hline 24 & 1 & -0.02 & -0.01 & -0.02 & 0.05 & -0.10 & 0.04 \\
\hline 25 & 1 & 0.29 & 0.18 & 0.17 & 0.14 & -0.08 & -0.03 \\
\hline 26 & 6 & 0.50 & 0.23 & 0.27 & 0.15 & -0.06 & -0.03 \\
\hline 27 & 1 & 0.28 & 0.18 & 0.21 & 0.22 & -0.04 & -0.02 \\
\hline 28 & 6 & 0.05 & 0.07 & 0.07 & 0.11 & -0.07 & -0.03 \\
\hline 29 & 1 & -0.04 & -0.02 & 0.05 & 0.14 & -0.05 & -0.02 \\
\hline 30 & 6 & -0.08 & -0.06 & -0.04 & 0.03 & -0.11 & -0.04 \\
\hline 31 & 1 & 0.09 & 0.06 & -0.11 & 0.04 & -0.14 & -0.06 \\
\hline 32 & 1 & -0.08 & 0.05 & -0.04 & 0.01 & -0.14 & -0.06 \\
\hline 33 & 50 & 0.03 & 0.00 & 0.02 & -0.08 & -0.03 & 0.01 \\
\hline 34 & 6 & 0.00 & -0.03 & -0.01 & -0.05 & 0.10 & 0.02 \\
\hline 35 & 6 & -0.05 & -0.02 & 0.01 & -0.14 & -0.06 & 0.02 \\
\hline 36 & 6 & -0.05 & -0.08 & -0.01 & -0.16 & -0.07 & 0.03 \\
\hline 37 & 1 & -0.01 & 0.01 & -0.03 & -0.01 & 0.12 & 0.01 \\
\hline 38 & 1 & -0.06 & -0.09 & -0.01 & -0.02 & 0.12 & 0.01 \\
\hline 39 & 1 & 0.02 & -0.04 & 0.05 & -0.10 & 0.13 & 0.05 \\
\hline 40 & 1 & -0.06 & -0.01 & 0.00 & -0.25 & 0.00 & 0.04 \\
\hline 41 & 1 & -0.03 & -0.02 & -0.01 & -0.05 & -0.02 & 0.01 \\
\hline 42 & 1 & -0.05 & -0.04 & 0.00 & -0.16 & -0.18 & 0.03 \\
\hline 43 & 1 & -0.05 & -0.09 & -0.01 & -0.18 & -0.16 & 0.01 \\
\hline 44 & 1 & -0.03 & -0.08 & -0.01 & -0.10 & -0.04 & 0.02 \\
\hline 45 & 1 & -0.05 & -0.07 & -0.01 & -0.23 & -0.03 & 0.08 \\
\hline
\end{tabular}

\begin{tabular}{crr}
\multicolumn{1}{c}{$X$} & \multicolumn{1}{c}{$Y$} & \multicolumn{1}{c}{$Z$} \\
-0.06 & 0.19 & \multicolumn{1}{c}{-0.02} \\
-0.03 & 0.10 & -0.02 \\
-0.03 & 0.06 & -0.04 \\
-0.04 & 0.11 & -0.06 \\
-0.06 & 0.19 & -0.06 \\
-0.07 & 0.24 & -0.04 \\
-0.06 & 0.22 & -0.01 \\
-0.07 & 0.23 & -0.07 \\
-0.09 & 0.30 & -0.04 \\
0.00 & -0.02 & -0.05 \\
0.02 & -0.07 & -0.05 \\
0.01 & -0.06 & -0.05 \\
-0.01 & -0.07 & -0.06 \\
0.01 & -0.06 & -0.05 \\
-0.02 & 0.05 & 0.00 \\
-0.03 & 0.06 & -0.09 \\
-0.03 & 0.08 & -0.01 \\
-0.02 & 0.04 & 0.06 \\
-0.03 & 0.09 & -0.07 \\
-0.04 & 0.05 & -0.14 \\
0.01 & -0.03 & -0.04 \\
0.01 & -0.04 & -0.06 \\
0.00 & -0.07 & -0.10 \\
0.01 & -0.10 & 0.01 \\
0.00 & -0.07 & -0.08 \\
0.00 & -0.03 & -0.08 \\
0.00 & -0.02 & -0.11 \\
0.01 & 0.00 & -0.03 \\
0.02 & 0.04 & -0.03 \\
0.02 & -0.01 & 0.01 \\
0.03 & 0.03 & 0.02 \\
0.00 & -0.05 & 0.00 \\
0.02 & -0.02 & 0.12 \\
0.01 & 0.04 & 0.08 \\
0.00 & -0.11 & 0.15 \\
0.01 & 0.05 & 0.20 \\
0.01 & 0.11 & 0.05 \\
0.01 & -0.02 & 0.04 \\
0.01 & 0.05 & 0.15 \\
0.00 & -0.12 & 0.19 \\
0.00 & -0.14 & 0.12 \\
0.00 & -0.13 & 0.15 \\
0.00 & 0.01 & 0.20 \\
0.02 & 0.12 & 0.18 \\
0.00 & 0.05 & 0.27
\end{tabular}


thermodynamics:

Sum of electronic and zero-point Energies=

$-809.696715$

Sum of electronic and thermal Enthalpies=

$-809.667442$

Sum of electronic and thermal Free Energies=

$-809.761201$

Total free energy in solution:

with all non electrostatic terms

$(\mathrm{a} . \mathrm{u})=.\quad-810.069547$

Optimized geometry, SCF energy, three lower frequencies, thermochemistry and PCM energy for B3.

\begin{tabular}{|c|c|c|c|c|c|}
\hline \multirow{2}{*}{$\begin{array}{l}\text { Center } \\
\text { Number } \\
-------\end{array}$} & \multirow{2}{*}{$\begin{array}{l}\text { Atomic } \\
\text { Number }\end{array}$} & \multirow{2}{*}{$\begin{array}{c}\text { Atomic } \\
\text { Type }\end{array}$} & \multicolumn{3}{|c|}{ Coordinates (Angstroms) } \\
\hline & & & $\mathrm{X}$ & Y & $\mathrm{Z}$ \\
\hline 1 & 6 & 0 & 0.892323 & 0.907948 & 0.037590 \\
\hline 2 & 6 & 0 & 0.695220 & 0.995312 & 1.420465 \\
\hline 3 & 6 & 0 & 1.261796 & 0.031753 & 2.279513 \\
\hline 4 & 6 & 0 & 2.043533 & -1.000528 & 1.720539 \\
\hline 5 & 6 & 0 & 2.263313 & -1.045773 & 0.338973 \\
\hline 6 & 6 & 0 & 1.682335 & -0.102059 & -0.501200 \\
\hline 7 & 1 & 0 & 0.430113 & 1.641799 & -0.618466 \\
\hline 8 & 1 & 0 & 2.887323 & -1.831824 & -0.079659 \\
\hline 9 & 1 & 0 & 1.843918 & -0.153789 & -1.573649 \\
\hline 10 & 46 & 0 & 0.935758 & 0.094250 & 4.245943 \\
\hline 11 & 6 & 0 & -0.102832 & 2.135573 & 1.966820 \\
\hline 12 & 6 & 0 & 2.631290 & -2.067521 & 2.589923 \\
\hline 13 & 1 & 0 & -0.864100 & 2.506104 & 1.277446 \\
\hline 14 & 1 & 0 & 0.520431 & 2.969114 & 2.303395 \\
\hline 15 & 1 & 0 & 3.556240 & -2.490818 & 2.192648 \\
\hline 16 & 1 & 0 & 1.926036 & -2.876003 & 2.803511 \\
\hline 17 & 34 & 0 & 3.047522 & -1.215140 & 4.372304 \\
\hline 18 & 34 & 0 & -1.049672 & 1.479293 & 3.629401 \\
\hline 19 & 1 & 0 & 2.608705 & -2.354888 & 5.231212 \\
\hline 20 & 1 & 0 & -0.793673 & 2.691877 & 4.466042 \\
\hline 21 & 8 & 0 & 0.521989 & 0.040630 & 6.397441 \\
\hline 22 & 6 & 0 & 0.806504 & 1.205692 & 7.177824 \\
\hline 23 & 50 & 0 & -0.852665 & -1.300264 & 7.087309 \\
\hline 24 & 1 & 0 & 0.209819 & 2.052051 & 6.807790 \\
\hline 25 & 6 & 0 & 2.284894 & 1.574269 & 7.160610 \\
\hline 26 & 1 & 0 & 0.497801 & 1.023837 & 8.218259 \\
\hline 27 & 6 & 0 & -0.165786 & -1.817708 & 9.043553 \\
\hline 28 & 6 & 0 & -2.717404 & -0.254151 & 7.060660 \\
\hline 29 & 6 & 0 & -0.707755 & -2.880594 & 5.657147 \\
\hline 30 & 1 & 0 & 2.605200 & 1.798233 & 6.133740 \\
\hline 31 & 1 & 0 & 2.377596 & 2.514924 & 7.726480 \\
\hline 32 & 6 & 0 & 3.177366 & 0.535159 & 7.776060 \\
\hline 33 & 1 & 0 & -0.730150 & -2.682587 & 9.408230 \\
\hline 34 & 1 & 0 & 0.894245 & -2.087044 & 9.020430 \\
\hline 35 & 1 & 0 & -0.309146 & -0.998233 & 9.753539 \\
\hline 36 & 1 & 0 & -2.647876 & 0.681887 & 7.623082 \\
\hline 37 & 1 & 0 & -3.032628 & -0.033448 & 6.036497 \\
\hline 38 & 1 & 0 & -3.491505 & -0.871607 & 7.528532 \\
\hline
\end{tabular}




$\begin{array}{ll}39 & 1 \\ 40 & 1 \\ 41 & 1 \\ 42 & 1 \\ 43 & 6 \\ 44 & 1 \\ 45 & 1\end{array}$

$\begin{array}{rrr}0.183347 & -3.490748 & 5.833480 \\ -1.585279 & -3.530530 & 5.741321 \\ -0.677096 & -2.482706 & 4.637783 \\ 2.818378 & 0.089957 & 8.706187 \\ 4.373796 & 0.174723 & 7.306885 \\ 4.996797 & -0.546521 & 7.829138 \\ 4.793962 & 0.617800 & 6.406020\end{array}$

$\mathrm{HF}=-810.1150422$

\begin{tabular}{|c|c|c|c|c|}
\hline & & & ${ }^{1}{ }_{\mathrm{A}}$ & \\
\hline Frequ & uencies & -- & 20.530 & \\
\hline Red. & masses & -- & 3.869 & \\
\hline Frc & consts & -- & 0.001 & \\
\hline IR Ir & nten & -- & 0.024 & \\
\hline Atom & AN & $\mathrm{X}$ & $\mathrm{Y}$ & Z \\
\hline 1 & 6 & -0.06 & -0.01 & 0.02 \\
\hline 2 & 6 & -0.04 & -0.01 & 0.02 \\
\hline 3 & 6 & -0.01 & 0.00 & 0.02 \\
\hline 4 & 6 & -0.02 & 0.00 & 0.00 \\
\hline 5 & 6 & -0.05 & -0.01 & 0.00 \\
\hline 6 & 6 & -0.07 & -0.01 & 0.01 \\
\hline 7 & 1 & -0.08 & -0.01 & 0.03 \\
\hline 8 & 1 & -0.06 & 0.00 & -0.01 \\
\hline 9 & 1 & -0.09 & -0.01 & 0.00 \\
\hline 10 & 46 & 0.02 & -0.01 & 0.02 \\
\hline 11 & 6 & -0.03 & -0.01 & 0.04 \\
\hline 12 & 6 & 0.00 & 0.00 & -0.01 \\
\hline 13 & 1 & -0.04 & -0.01 & 0.05 \\
\hline 14 & 1 & -0.02 & -0.01 & 0.03 \\
\hline 15 & 1 & 0.00 & 0.01 & -0.02 \\
\hline 16 & 1 & 0.01 & 0.00 & 0.01 \\
\hline 17 & 34 & 0.03 & 0.01 & -0.02 \\
\hline 18 & 34 & 0.00 & -0.01 & 0.05 \\
\hline 19 & 1 & 0.07 & 0.01 & 0.00 \\
\hline 20 & 1 & 0.01 & -0.01 & 0.05 \\
\hline 21 & 8 & 0.04 & -0.03 & 0.03 \\
\hline 22 & 6 & 0.05 & -0.03 & 0.04 \\
\hline 23 & 50 & -0.03 & 0.01 & -0.03 \\
\hline 24 & 1 & 0.03 & -0.04 & 0.07 \\
\hline 25 & 6 & 0.04 & -0.02 & 0.00 \\
\hline 26 & 1 & 0.08 & -0.06 & 0.04 \\
\hline 27 & 6 & -0.21 & 0.12 & 0.06 \\
\hline 28 & 6 & -0.03 & 0.00 & -0.26 \\
\hline 29 & 6 & 0.12 & -0.06 & 0.06 \\
\hline 30 & 1 & 0.01 & 0.01 & 0.00 \\
\hline 31 & 1 & 0.04 & -0.02 & 0.02 \\
\hline 32 & 6 & 0.07 & -0.02 & -0.04 \\
\hline 33 & 1 & -0.23 & 0.12 & 0.04 \\
\hline 34 & 1 & -0.20 & 0.15 & 0.19 \\
\hline 35 & 1 & -0.32 & 0.15 & 0.01 \\
\hline 36 & 1 & -0.07 & 0.05 & -0.34 \\
\hline 37 & 1 & 0.04 & -0.10 & -0.30 \\
\hline 38 & 1 & -0.06 & 0.04 & -0.25 \\
\hline 39 & 1 & 0.16 & 0.01 & 0.12 \\
\hline 40 & 1 & 0.16 & -0.12 & 0.08 \\
\hline 41 & 1 & 0.12 & -0.12 & 0.04 \\
\hline 42 & 1 & 0.09 & -0.05 & -0.05 \\
\hline 43 & 6 & 0.07 & 0.02 & -0.07 \\
\hline 44 & 1 & 0.10 & 0.02 & -0.10 \\
\hline
\end{tabular}

\begin{tabular}{|c|c|c|c|c|c|}
\hline \multicolumn{3}{|c|}{2} & \multicolumn{3}{|c|}{3} \\
\hline \multicolumn{3}{|c|}{ A } & \multicolumn{3}{|c|}{ A } \\
\hline & \multicolumn{2}{|c|}{21.4967} & & \multicolumn{2}{|c|}{25.0752} \\
\hline & \multicolumn{2}{|c|}{5.0675} & & \multicolumn{2}{|c|}{4.6010} \\
\hline & \multicolumn{2}{|c|}{0.0014} & & \multicolumn{2}{|c|}{0.0017} \\
\hline & \multicolumn{2}{|c|}{0.0409} & & \multicolumn{2}{|c|}{0.0173} \\
\hline $\mathrm{X}$ & Y & Z & $\mathrm{X}$ & $\mathrm{Y}$ & Z \\
\hline 0.01 & -0.04 & 0.01 & 0.08 & 0.08 & 0.00 \\
\hline 0.03 & -0.01 & 0.01 & 0.05 & 0.06 & -0.01 \\
\hline 0.00 & -0.02 & 0.02 & 0.00 & 0.02 & -0.01 \\
\hline-0.05 & -0.05 & 0.03 & -0.01 & 0.01 & -0.02 \\
\hline-0.06 & -0.09 & 0.03 & 0.02 & 0.03 & -0.01 \\
\hline-0.04 & -0.08 & 0.02 & 0.07 & 0.07 & -0.01 \\
\hline 0.03 & -0.03 & 0.01 & 0.12 & 0.11 & 0.00 \\
\hline-0.10 & -0.12 & 0.04 & 0.01 & 0.03 & -0.01 \\
\hline-0.05 & -0.10 & 0.02 & 0.09 & 0.09 & 0.00 \\
\hline 0.00 & 0.01 & 0.02 & -0.03 & 0.00 & -0.02 \\
\hline 0.08 & 0.03 & 0.01 & 0.07 & 0.07 & 0.00 \\
\hline-0.07 & -0.06 & 0.04 & -0.06 & -0.02 & -0.02 \\
\hline 0.09 & 0.06 & 0.01 & 0.10 & 0.10 & -0.01 \\
\hline 0.11 & 0.01 & 0.00 & 0.08 & 0.04 & 0.04 \\
\hline-0.09 & -0.10 & 0.04 & -0.07 & -0.05 & -0.01 \\
\hline-0.09 & -0.03 & 0.07 & -0.09 & 0.00 & -0.04 \\
\hline-0.02 & -0.03 & 0.02 & -0.06 & -0.06 & -0.01 \\
\hline 0.06 & 0.09 & 0.02 & 0.01 & 0.05 & -0.04 \\
\hline-0.04 & -0.01 & 0.05 & -0.10 & -0.05 & -0.03 \\
\hline 0.11 & 0.08 & 0.00 & 0.00 & 0.03 & -0.01 \\
\hline-0.01 & -0.02 & 0.02 & -0.03 & 0.02 & -0.02 \\
\hline-0.06 & -0.02 & 0.04 & -0.03 & 0.03 & -0.04 \\
\hline 0.00 & -0.05 & -0.03 & 0.04 & -0.02 & 0.04 \\
\hline-0.15 & -0.05 & 0.10 & -0.02 & 0.03 & -0.07 \\
\hline-0.09 & 0.11 & -0.03 & -0.03 & 0.02 & -0.03 \\
\hline 0.01 & -0.08 & 0.05 & -0.05 & 0.06 & -0.04 \\
\hline 0.01 & -0.10 & -0.04 & 0.02 & 0.16 & 0.10 \\
\hline-0.01 & -0.06 & 0.00 & -0.03 & -0.15 & -0.08 \\
\hline 0.01 & -0.01 & -0.07 & 0.18 & -0.11 & 0.15 \\
\hline-0.17 & 0.14 & -0.05 & -0.01 & -0.02 & -0.03 \\
\hline-0.14 & 0.11 & -0.03 & -0.03 & 0.03 & -0.06 \\
\hline 0.03 & 0.17 & -0.10 & -0.05 & 0.02 & 0.01 \\
\hline 0.11 & -0.20 & -0.12 & -0.01 & 0.21 & 0.18 \\
\hline 0.04 & 0.02 & -0.04 & 0.01 & 0.12 & 0.13 \\
\hline-0.10 & -0.17 & 0.01 & 0.04 & 0.23 & 0.01 \\
\hline-0.02 & -0.09 & 0.05 & -0.12 & -0.13 & -0.12 \\
\hline 0.01 & -0.01 & 0.00 & 0.00 & -0.22 & -0.11 \\
\hline-0.01 & -0.09 & -0.05 & -0.01 & -0.19 & -0.09 \\
\hline-0.01 & -0.03 & -0.07 & 0.26 & 0.01 & 0.16 \\
\hline-0.01 & 0.01 & -0.11 & 0.27 & -0.22 & 0.24 \\
\hline 0.03 & 0.02 & -0.06 & 0.08 & -0.18 & 0.12 \\
\hline 0.12 & 0.14 & -0.08 & -0.06 & 0.07 & 0.03 \\
\hline 0.03 & 0.27 & -0.17 & -0.06 & -0.03 & 0.02 \\
\hline 0.12 & 0.32 & -0.22 & -0.08 & -0.02 & 0.05 \\
\hline-0.07 & 0.31 & -0.20 & -0.05 & -0.08 & 0.00 \\
\hline
\end{tabular}

$-809.756196$

$-809.726798$

$-809.820107$ 
Total free energy in solution:

with all non electrostatic terms

$(\mathrm{a} . \mathrm{u})=.\quad-810.132451$

\section{biscarbene complex}

Optimized geometry, SCF energy, three lower frequencies, thermochemistry and PCM energy for B2.

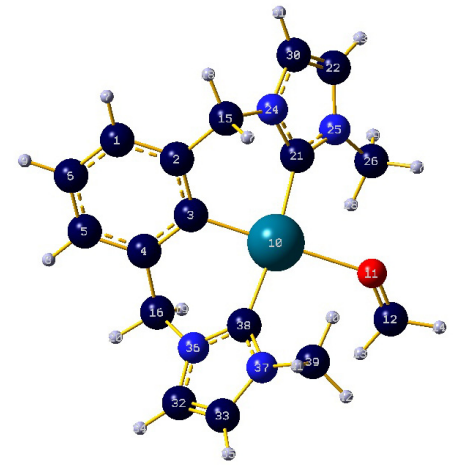

\begin{tabular}{|c|c|c|c|c|c|}
\hline \multirow{2}{*}{$\begin{array}{l}\text { Center } \\
\text { Number }\end{array}$} & \multirow{2}{*}{$\begin{array}{l}\text { Atomic } \\
\text { Number }\end{array}$} & \multirow{2}{*}{$\begin{array}{l}\text { Atomic } \\
\text { Type }\end{array}$} & \multicolumn{3}{|c|}{ Coordinates (Angstroms) } \\
\hline & & & $\mathrm{X}$ & Y & Z \\
\hline 1 & 6 & 0 & 0.003531 & 0.000475 & 0.028064 \\
\hline 2 & 6 & 0 & -0.001869 & -0.006680 & 1.426553 \\
\hline 3 & 6 & 0 & 1.206713 & -0.003410 & 2.139298 \\
\hline 4 & 6 & 0 & 2.413997 & 0.003320 & 1.422070 \\
\hline 5 & 6 & 0 & 2.404750 & 0.016268 & 0.023972 \\
\hline 6 & 6 & 0 & 1.202789 & 0.014981 & -0.676442 \\
\hline 7 & 1 & 0 & -0.940388 & -0.000604 & -0.513141 \\
\hline 8 & 1 & 0 & 3.346884 & 0.021037 & -0.520398 \\
\hline 9 & 1 & 0 & 1.200860 & 0.023081 & -1.762351 \\
\hline 10 & 46 & 0 & 1.217883 & -0.001730 & 4.136403 \\
\hline 11 & 8 & 0 & 1.237998 & 0.048844 & 6.392041 \\
\hline 12 & 6 & 0 & 2.203165 & 0.112286 & 7.132234 \\
\hline 13 & 1 & 0 & 3.235116 & 0.103192 & 6.746668 \\
\hline 14 & 1 & 0 & 2.060593 & 0.179664 & 8.221999 \\
\hline 15 & 6 & 0 & -1.321664 & 0.000562 & 2.159853 \\
\hline 16 & 6 & 0 & 3.736339 & -0.039076 & 2.152001 \\
\hline 17 & 1 & 0 & -1.491450 & -0.944838 & 2.688044 \\
\hline 18 & 1 & 0 & -2.153387 & 0.148180 & 1.466866 \\
\hline 19 & 1 & 0 & 3.920122 & 0.887337 & 2.707904 \\
\hline 20 & 1 & 0 & 4.566135 & -0.180588 & 1.455467 \\
\hline 21 & 6 & 0 & -0.365400 & 1.293905 & 4.024323 \\
\hline 22 & 6 & 0 & -1.946967 & 2.885783 & 4.245342 \\
\hline 23 & 1 & 0 & -2.409892 & 3.772559 & 4.650996 \\
\hline 24 & 7 & 0 & -1.365317 & 1.086449 & 3.141380 \\
\hline 25 & 7 & 0 & -0.729050 & 2.411738 & 4.698764 \\
\hline 26 & 6 & 0 & 0.041436 & 3.047134 & 5.754144 \\
\hline 27 & 1 & 0 & -0.311441 & 2.727372 & 6.738975 \\
\hline 28 & 1 & 0 & 1.091067 & 2.779154 & 5.632559 \\
\hline 29 & 1 & 0 & -0.056369 & 4.131844 & 5.669536 \\
\hline 30 & 6 & 0 & -2.347597 & 2.046705 & 3.256037 \\
\hline 31 & 1 & 0 & -3.229411 & 2.057452 & 2.633709 \\
\hline 32 & 6 & 0 & 4.665202 & -2.192340 & 3.140458 \\
\hline 33 & 6 & 0 & 4.219915 & -3.055716 & 4.089720 \\
\hline 34 & 1 & 0 & 5.535559 & -2.231361 & 2.503070 \\
\hline 35 & 1 & 0 & 4.622613 & -3.996106 & 4.434762 \\
\hline 36 & 7 & 0 & 3.757849 & -1.155188 & 3.102260 \\
\hline 37 & 7 & 0 & 3.049325 & -2.518489 & 4.597098 \\
\hline 38 & 6 & 0 & 2.758146 & -1.340691 & 3.990235 \\
\hline 39 & 6 & 0 & 2.209808 & -3.190502 & 5.57615 \\
\hline
\end{tabular}




$$
\begin{aligned}
& 40 \\
& 41 \\
& 42
\end{aligned}
$$

40
41
42

1
1
1

\section{$1.293602-2.614250$ \\ $1.950213-4.190055$}

$2.730424-3.279923$
5.705080

5.217257

6.534658

$\mathrm{HF}=-1079.7111038$

\begin{tabular}{|c|c|c|c|c|c|c|c|c|c|c|}
\hline & \multicolumn{3}{|c|}{1} & \multicolumn{3}{|c|}{2} & \multicolumn{3}{|c|}{3} \\
\hline & & \multicolumn{3}{|c|}{ A } & \multicolumn{3}{|c|}{ A } & \multicolumn{3}{|c|}{ A } \\
\hline \multicolumn{2}{|c|}{ Frequencies } & $5--$ & \multicolumn{2}{|c|}{39.9809} & \multicolumn{3}{|c|}{52.7802} & \multicolumn{3}{|c|}{59.8671} \\
\hline \multicolumn{2}{|c|}{ Red. masses } & $5--$ & \multicolumn{2}{|c|}{4.8075} & \multicolumn{3}{|c|}{3.9467} & \multicolumn{3}{|c|}{3.3826} \\
\hline \multicolumn{2}{|c|}{ Frc consts } & -- & \multicolumn{2}{|c|}{0.0045} & \multicolumn{3}{|c|}{0.0065} & \multicolumn{3}{|c|}{0.0071} \\
\hline IR In & nten & -- & \multicolumn{2}{|c|}{0.9591} & & 1.468 & & & 7.334 & \\
\hline Atom & AN & $\mathrm{X}$ & $\mathrm{Y}$ & Z & $\mathrm{X}$ & $\mathrm{Y}$ & Z & $\mathrm{X}$ & $\mathrm{Y}$ & Z \\
\hline 1 & 6 & 0.05 & 0.03 & -0.02 & 0.01 & -0.07 & 0.05 & -0.01 & 0.12 & 0.00 \\
\hline 2 & 6 & 0.04 & 0.02 & -0.02 & 0.02 & -0.06 & 0.05 & 0.00 & 0.02 & 0.00 \\
\hline 3 & 6 & 0.04 & 0.01 & -0.01 & 0.02 & 0.01 & 0.04 & 0.00 & -0.02 & 0.00 \\
\hline 4 & 6 & 0.04 & -0.02 & 0.00 & 0.02 & 0.06 & 0.04 & 0.00 & 0.01 & 0.00 \\
\hline 5 & 6 & 0.05 & -0.01 & 0.00 & 0.01 & 0.06 & 0.04 & -0.01 & 0.12 & 0.00 \\
\hline 6 & 6 & 0.06 & 0.02 & -0.01 & 0.01 & 0.00 & 0.05 & -0.01 & 0.18 & 0.00 \\
\hline 7 & 1 & 0.06 & 0.05 & -0.03 & 0.01 & -0.12 & 0.05 & -0.01 & 0.16 & 0.01 \\
\hline 8 & 1 & 0.06 & -0.03 & 0.00 & 0.01 & 0.11 & 0.04 & -0.01 & 0.15 & -0.01 \\
\hline 9 & 1 & 0.06 & 0.03 & -0.01 & 0.01 & 0.00 & 0.05 & -0.01 & 0.27 & 0.00 \\
\hline 10 & 46 & 0.03 & 0.05 & -0.01 & 0.02 & 0.02 & 0.04 & 0.00 & -0.04 & 0.00 \\
\hline 11 & 8 & 0.12 & 0.14 & -0.01 & 0.01 & 0.04 & 0.04 & -0.02 & -0.06 & 0.00 \\
\hline 12 & 6 & 0.17 & 0.12 & -0.08 & 0.00 & 0.00 & 0.05 & -0.06 & 0.30 & 0.02 \\
\hline 13 & 1 & 0.14 & 0.02 & -0.14 & 0.00 & -0.05 & 0.06 & -0.04 & 0.71 & 0.04 \\
\hline 14 & 1 & 0.24 & 0.20 & -0.07 & -0.01 & 0.01 & 0.05 & -0.10 & 0.20 & 0.02 \\
\hline 15 & 6 & 0.04 & 0.00 & -0.04 & 0.03 & -0.12 & 0.07 & 0.01 & -0.04 & 0.02 \\
\hline 16 & 6 & 0.04 & -0.06 & 0.00 & 0.01 & 0.08 & 0.06 & 0.00 & -0.05 & -0.02 \\
\hline 17 & 1 & 0.04 & -0.02 & -0.06 & 0.09 & -0.10 & 0.11 & 0.05 & -0.05 & 0.01 \\
\hline 18 & 1 & 0.05 & 0.01 & -0.05 & 0.00 & -0.19 & 0.08 & -0.01 & -0.06 & 0.03 \\
\hline 19 & 1 & 0.08 & -0.07 & 0.00 & -0.03 & 0.06 & 0.10 & 0.05 & -0.06 & -0.01 \\
\hline 20 & 1 & 0.04 & -0.09 & 0.00 & 0.03 & 0.13 & 0.07 & -0.01 & -0.07 & -0.03 \\
\hline 21 & 6 & -0.01 & 0.00 & 0.00 & 0.00 & 0.00 & 0.01 & 0.00 & -0.03 & 0.00 \\
\hline 22 & 6 & -0.13 & -0.13 & 0.09 & -0.01 & 0.00 & -0.10 & 0.01 & -0.01 & -0.02 \\
\hline 23 & 1 & -0.19 & -0.18 & 0.14 & -0.02 & 0.02 & -0.16 & 0.02 & 0.00 & -0.04 \\
\hline 24 & 7 & 0.00 & -0.03 & -0.01 & -0.01 & -0.08 & 0.03 & -0.01 & -0.05 & 0.02 \\
\hline 25 & 7 & -0.09 & -0.06 & 0.06 & -0.01 & 0.04 & -0.07 & 0.02 & -0.01 & -0.02 \\
\hline 26 & 6 & -0.14 & -0.06 & 0.09 & 0.00 & 0.14 & -0.14 & 0.04 & 0.03 & -0.07 \\
\hline 27 & 1 & -0.06 & -0.21 & 0.07 & -0.07 & 0.33 & -0.11 & 0.06 & 0.09 & -0.05 \\
\hline 28 & 1 & -0.11 & 0.10 & 0.03 & -0.02 & 0.01 & -0.05 & 0.04 & 0.02 & -0.08 \\
\hline 29 & 1 & -0.30 & -0.07 & 0.21 & 0.12 & 0.13 & -0.32 & 0.06 & 0.03 & -0.12 \\
\hline 30 & 6 & -0.07 & -0.11 & 0.05 & -0.01 & -0.08 & -0.04 & 0.00 & -0.04 & 0.01 \\
\hline 31 & 1 & -0.08 & -0.14 & 0.06 & -0.02 & -0.13 & -0.03 & -0.01 & -0.05 & 0.02 \\
\hline 32 & 6 & -0.08 & -0.13 & -0.01 & -0.05 & -0.02 & -0.10 & -0.02 & -0.06 & -0.01 \\
\hline 33 & 6 & -0.13 & -0.10 & -0.01 & -0.08 & -0.08 & -0.16 & 0.00 & -0.03 & 0.01 \\
\hline 34 & 1 & -0.09 & -0.18 & -0.02 & -0.06 & -0.02 & -0.11 & -0.03 & -0.07 & -0.03 \\
\hline 35 & 1 & -0.19 & -0.12 & -0.02 & -0.13 & -0.13 & -0.24 & 0.00 & -0.03 & 0.03 \\
\hline 36 & 7 & -0.01 & -0.06 & 0.00 & 0.01 & 0.04 & 0.01 & -0.02 & -0.06 & -0.03 \\
\hline 37 & 7 & -0.09 & -0.02 & 0.00 & -0.05 & -0.05 & -0.10 & 0.01 & -0.02 & 0.02 \\
\hline 38 & 6 & -0.01 & 0.00 & 0.01 & 0.01 & 0.01 & 0.01 & 0.00 & -0.04 & 0.00 \\
\hline 39 & 6 & -0.13 & 0.04 & 0.01 & -0.07 & -0.09 & -0.14 & 0.04 & 0.02 & 0.07 \\
\hline 40 & 1 & -0.08 & 0.11 & 0.02 & -0.01 & -0.01 & -0.04 & 0.04 & 0.02 & 0.08 \\
\hline 41 & 1 & -0.20 & 0.06 & 0.01 & -0.19 & -0.02 & -0.25 & 0.03 & 0.00 & 0.13 \\
\hline 42 & 1 & -0.12 & 0.00 & 0.00 & -0.04 & -0.25 & -0.17 & 0.07 & 0.06 & 0.06 \\
\hline
\end{tabular}

thermodynamics:

Sum of electronic and zero-point Energies=

$-1079.369624$

Sum of electronic and thermal Enthalpies=

$-1079.346737$

Sum of electronic and thermal Free Energies=

$-1079.420567$

Total free energy in solution:

with all non electrostatic terms

$(\mathrm{a} \cdot \mathrm{u} \cdot)=-1079.745267$

Optimized geometry, SCF energy, three lower frequencies, thermochemistry and PCM energy for $\mathbf{T S}_{\mathbf{B 2} \mathbf{B} 3}$. 




\begin{tabular}{|c|c|c|c|c|c|}
\hline \multirow{2}{*}{$\begin{array}{l}\text { Center } \\
\text { Number }\end{array}$} & \multirow{2}{*}{$\begin{array}{l}\text { Atomic } \\
\text { Number }\end{array}$} & \multirow{2}{*}{$\begin{array}{l}\text { Atomic } \\
\text { Type }\end{array}$} & \multicolumn{3}{|c|}{ Coordinates (Angstroms) } \\
\hline & & & $\mathrm{x}$ & $\mathrm{Y}$ & Z \\
\hline 1 & 6 & 0 & -0.055957 & -0.049341 & 0.039013 \\
\hline 2 & 6 & 0 & -0.020833 & -0.002247 & 1.435979 \\
\hline 3 & 6 & 0 & 1.204739 & -0.001977 & 2.119980 \\
\hline 4 & 6 & 0 & 2.389592 & -0.050770 & 1.367484 \\
\hline 5 & 6 & 0 & 2.345120 & -0.090405 & -0.029082 \\
\hline 6 & 6 & 0 & 1.124222 & -0.090348 & -0.697246 \\
\hline 7 & 1 & 0 & -1.014326 & -0.047903 & -0.476702 \\
\hline 8 & 1 & 0 & 3.272272 & -0.129208 & -0.597743 \\
\hline 9 & 1 & 0 & 1.092850 & -0.123790 & -1.782360 \\
\hline 10 & 46 & 0 & 1.263956 & 0.066816 & 4.121321 \\
\hline 11 & 8 & 0 & 1.299452 & 0.234424 & 6.351262 \\
\hline 12 & 6 & 0 & 2.336434 & 0.717170 & 6.912295 \\
\hline 13 & 1 & 0 & 3.244895 & 0.883910 & 6.315315 \\
\hline 14 & 1 & 0 & 2.196684 & 1.447425 & 7.721681 \\
\hline 15 & 6 & 0 & -1.311597 & 0.087666 & 2.215983 \\
\hline 16 & 6 & 0 & 3.722005 & -0.116326 & 2.078329 \\
\hline 17 & 1 & 0 & -1.482321 & -0.812793 & 2.816729 \\
\hline 18 & 1 & 0 & -2.169048 & 0.214160 & 1.550327 \\
\hline 19 & 1 & 0 & 3.933199 & 0.808006 & 2.627261 \\
\hline 20 & 1 & 0 & 4.542050 & -0.286246 & 1.376277 \\
\hline 21 & 6 & 0 & -0.235055 & 1.456034 & 3.954387 \\
\hline 22 & 6 & 0 & -1.661361 & 3.204948 & 4.007503 \\
\hline 23 & 1 & 0 & -2.030629 & 4.172266 & 4.312922 \\
\hline 24 & 7 & 0 & -1.275332 & 1.241531 & 3.117847 \\
\hline 25 & 7 & 0 & -0.479693 & 2.674575 & 4.495651 \\
\hline 26 & 6 & 0 & 0.404262 & 3.388016 & 5.399278 \\
\hline 27 & 1 & 0 & 0.603227 & 4.388361 & 5.004928 \\
\hline 28 & 1 & 0 & -0.044509 & 3.473856 & 6.392899 \\
\hline 29 & 1 & 0 & 1.341589 & 2.839123 & 5.467150 \\
\hline 30 & 6 & 0 & -2.162204 & 2.297348 & 3.131459 \\
\hline 31 & 1 & 0 & -3.057045 & 2.315962 & 2.528101 \\
\hline 32 & 6 & 0 & 4.530897 & -2.338189 & 3.017649 \\
\hline 33 & 6 & 0 & 4.040945 & -3.188537 & 3.955931 \\
\hline 34 & 1 & 0 & 5.381713 & -2.427199 & 2.359305 \\
\hline 35 & 1 & 0 & 4.374434 & -4.166907 & 4.267357 \\
\hline 36 & 7 & 0 & 3.714509 & -1.226428 & 3.035866 \\
\hline 37 & 7 & 0 & 2.936281 & -2.567308 & 4.514118 \\
\hline 38 & 6 & 0 & 2.725866 & -1.353042 & 3.948276 \\
\hline 39 & 1 & 0 & 3.438012 & -1.387170 & 7.211697 \\
\hline 40 & 6 & 0 & 3.337865 & -0.650706 & 8.004498 \\
\hline 41 & 1 & 0 & 4.230806 & -0.065888 & 8.213927 \\
\hline 42 & 6 & 0 & 2.454103 & -0.882192 & 9.041496 \\
\hline 43 & 1 & 0 & 2.551212 & -0.245619 & 9.923581 \\
\hline 44 & 6 & 0 & 1.346475 & -1.794255 & 9.038548 \\
\hline 45 & 1 & 0 & 1.117229 & -2.174857 & 10.038360 \\
\hline 46 & 1 & 0 & 1.429601 & -2.610124 & 8.318232 \\
\hline 47 & 50 & 0 & -0.676155 & -0.823296 & 8.633962 \\
\hline 48 & 6 & 0 & -0.616008 & 1.306118 & 8.877124 \\
\hline 49 & 6 & 0 & -1.502414 & -1.531968 & 6.786553 \\
\hline
\end{tabular}




$\begin{array}{ll}50 & 6 \\ 51 & 1 \\ 52 & 1 \\ 53 & 1 \\ 54 & 1 \\ 55 & 1 \\ 56 & 1 \\ 57 & 1 \\ 58 & 1 \\ 59 & 1 \\ 60 & 6 \\ 61 & 1 \\ 62 & 1 \\ 63 & 1\end{array}$

-1.786594
-1.637389
-0.045049
-0.171058
-0.966774
-1.459067
-2.555700
-1.378356
-2.833241
-1.765043
2.028247
1.376991
1.420063
2.596007

-1.678276
1.681582
1.591545
1.765829
-1.106125
-2.624762
-1.235849
-1.356530
-1.358911
-2.772250
-3.205672
-2.438752
-3.956091
-3.691962
10.267705

8.998749

9.765951

7.993221

5.934625

6.739605

6.730718

11.231429

10.220434

10.229704

5.449502

5.869005

4.934833

6.247842
$\mathrm{HF}=-1320.0531492$

\begin{tabular}{|c|c|c|c|c|c|}
\hline \multicolumn{6}{|c|}{$\perp$} \\
\hline \multirow{2}{*}{\multicolumn{3}{|c|}{ Frequencies -- }} & \multicolumn{2}{|l|}{ A } & \\
\hline & & & \multicolumn{2}{|c|}{-241.4158} & \\
\hline \multicolumn{2}{|c|}{ Red. masses } & $5--$ & \multicolumn{2}{|c|}{8.5479} & \\
\hline \multicolumn{2}{|c|}{ Frc consts } & -- & \multirow{2}{*}{\multicolumn{2}{|c|}{$\begin{array}{r}0.2935 \\
363.1914\end{array}$}} & \\
\hline \multicolumn{2}{|c|}{ IR Inten } & -- & & & \\
\hline Atom & AN & $\mathrm{X}$ & $Y$ & Z & $\mathrm{X}$ \\
\hline 1 & 6 & 0.00 & 0.00 & 0.00 & -0.12 \\
\hline 2 & 6 & 0.00 & 0.00 & 0.00 & -0.07 \\
\hline 3 & 6 & 0.00 & 0.00 & -0.01 & -0.04 \\
\hline 4 & 6 & 0.00 & 0.00 & 0.00 & -0.07 \\
\hline 5 & 6 & 0.00 & 0.00 & 0.00 & -0.12 \\
\hline 6 & 6 & 0.00 & 0.00 & 0.00 & -0.15 \\
\hline 7 & 1 & 0.00 & 0.00 & 0.00 & -0.14 \\
\hline 8 & 1 & 0.00 & 0.00 & 0.00 & -0.15 \\
\hline 9 & 1 & 0.00 & 0.00 & 0.00 & -0.19 \\
\hline 10 & 46 & 0.00 & 0.00 & -0.03 & 0.03 \\
\hline 11 & 8 & -0.01 & 0.15 & -0.07 & 0.05 \\
\hline 12 & 6 & -0.20 & 0.29 & -0.16 & 0.03 \\
\hline 13 & 1 & 0.03 & -0.06 & 0.11 & 0.05 \\
\hline 14 & 1 & -0.14 & -0.06 & 0.15 & 0.02 \\
\hline 15 & 6 & 0.00 & 0.00 & 0.00 & -0.04 \\
\hline 16 & 6 & 0.00 & 0.00 & 0.00 & -0.04 \\
\hline 17 & 1 & 0.00 & 0.00 & 0.00 & -0.02 \\
\hline 18 & 1 & 0.00 & 0.00 & 0.00 & -0.07 \\
\hline 19 & 1 & 0.01 & 0.00 & -0.01 & -0.04 \\
\hline 20 & 1 & 0.00 & 0.00 & 0.00 & -0.07 \\
\hline 21 & 6 & 0.00 & 0.01 & -0.02 & 0.03 \\
\hline 22 & 6 & -0.01 & 0.00 & 0.00 & 0.05 \\
\hline 23 & 1 & -0.02 & -0.01 & 0.00 & 0.07 \\
\hline 24 & 7 & 0.00 & 0.00 & -0.01 & 0.00 \\
\hline 25 & 7 & 0.00 & 0.01 & -0.01 & 0.06 \\
\hline 26 & 6 & -0.01 & 0.03 & -0.02 & 0.11 \\
\hline 27 & 1 & -0.11 & 0.06 & 0.02 & 0.12 \\
\hline 28 & 1 & 0.05 & -0.06 & 0.01 & 0.15 \\
\hline 29 & 1 & 0.05 & 0.11 & -0.12 & 0.10 \\
\hline 30 & 6 & -0.01 & 0.00 & 0.00 & 0.01 \\
\hline 31 & 1 & -0.01 & -0.01 & 0.01 & -0.01 \\
\hline 32 & 6 & 0.00 & 0.00 & 0.00 & 0.03 \\
\hline 33 & 6 & 0.00 & 0.00 & 0.00 & 0.08 \\
\hline 34 & 1 & 0.01 & 0.00 & 0.01 & 0.01 \\
\hline 35 & 1 & 0.00 & 0.01 & 0.01 & 0.12 \\
\hline 36 & 7 & 0.00 & 0.00 & 0.00 & 0.01 \\
\hline 37 & 7 & 0.00 & 0.00 & 0.00 & 0.09 \\
\hline 38 & 6 & 0.00 & -0.01 & -0.01 & 0.04 \\
\hline 39 & 1 & 0.17 & -0.13 & 0.11 & 0.05 \\
\hline 40 & 6 & 0.38 & -0.39 & 0.36 & 0.01 \\
\hline 41 & 1 & 0.25 & -0.13 & 0.17 & 0.00 \\
\hline 42 & 6 & 0.09 & -0.01 & 0.04 & -0.04 \\
\hline 43 & 1 & 0.01 & 0.06 & 0.01 & -0.08 \\
\hline 44 & 6 & -0.12 & 0.09 & -0.06 & -0.04 \\
\hline 45 & 1 & 0.07 & -0.05 & -0.06 & -0.09 \\
\hline
\end{tabular}

\section{2}

17.0313

6.0873

0.0010

0.4433

$\mathrm{Y}$

$-0.06$

$-0.04$

$-0.02$

$-0.03$

$-0.05$

$-0.07$

$-0.08$

$-0.06$

$-0.09$

$-0.01$

$-0.01$

0.00

$-0.01$

0.01

$-0.02$

0.00

$-0.01$

$-0.03$

0.01

0.0

0.00

0.01

0.02

$-0.01$

0.01

0.02

0.00

0.06

0.00

0.00

0.00

0.03

0.04

0.04

0.05

0.05
0.01

0.02

0.01

0.01

0.01

0.02

0.01

0.01

$0.01-0.06$

$\begin{array}{lr}0.01 & -0.01\end{array}$
3

A

24.2609

4.9872

0.0017

0.8028

$\mathrm{X}$

$-0.03$

$-0.02$

$-0.01$

$-0.02$

$-0.03$

$-0.04$

$-0.04$

$-0.04$

$-0.05$

0.00

$-0.03$

$-0.07$

$-0.07$

$-0.13$

$-0.01$

$-0.02$

$-0.04$

$-0.01$

0.00

$-0.03$

0.04

0.11

0.14

0.03

0.09

0.11

0.06

0.15

0.13

0.07

0.07

$-0.03$

$-0.03$

$-0.04$

$-0.03$

$-0.02$

$-0.02$

$-0.02$

0.03

$-0.01$

$-0.04$

0.00

$-0.03$

0.05

0.08
$\mathrm{Y}$

$\begin{array}{ll}-0.02 & 0.00 \\ 0.01 & 0.00\end{array}$

$0.01 \quad 0.00$

$0.00-0.01$

$-0.05-0.02$

$\begin{array}{ll}-0.09 & -0.02\end{array}$

$-0.08-0.01$

$\begin{array}{ll}-0.01 & 0.01\end{array}$

$\begin{array}{ll}-0.12-0.03 \\ -0.11 & -0.01\end{array}$

$\begin{array}{ll}-0.11 & -0.01\end{array}$

$0.03-0.01$

$0.03-0.02$

0.090 .00

$0.15 \quad 0.02$

$0.08 \quad 0.00$

$\begin{array}{rr}0.07 & 0.00\end{array}$

$\begin{array}{lll}-0.06 & -0.03\end{array}$

$0.09 \quad 0.02$

$0.09 \quad 0.00$

$-0.05-0.06$

$-0.09-0.04$

$0.07-0.03$

$0.12 \quad-0.06$

$0.14-0.07$

$0.09-0.02$

$0.08-0.05$

$0.06-0.05$

$0.08-0.01$

$\begin{array}{ll}-0.01 & -0.02\end{array}$

$0.08-0.12$

$0.12-0.04$

$\begin{array}{ll}0.15 & -0.04\end{array}$

$\begin{array}{rr}-0.04 & 0.02\end{array}$

$0.00 \quad 0.05$

$\begin{array}{ll}-0.06 & 0.02\end{array}$

$\begin{array}{rr}0.00 & 0.08\end{array}$

$\begin{array}{ll}-0.03 & -0.01\end{array}$

0.020 .04

$0.00 \quad 0.00$

0.150 .03

$0.16 \quad 0.02$

$\begin{array}{ll}0.20 & 0.01\end{array}$

$\begin{array}{ll}0.12 & 0.02\end{array}$

0.140 .01

$\begin{array}{ll}0.05 & 0.04\end{array}$

$0.06 \quad 0.04$ 


$\begin{array}{rrrrrrrrrrr}46 & 1 & 0.03 & 0.04 & 0.00 & 0.00 & 0.01 & 0.01 & 0.10 & 0.05 & 0.05 \\ 47 & 50 & 0.01 & -0.02 & 0.03 & -0.02 & 0.01 & -0.10 & -0.01 & -0.08 & 0.02 \\ 48 & 6 & -0.03 & 0.00 & -0.01 & -0.04 & 0.01 & -0.08 & -0.13 & -0.06 & -0.05 \\ 49 & 6 & -0.02 & 0.03 & 0.00 & 0.04 & 0.01 & -0.13 & 0.04 & -0.15 & 0.03 \\ 50 & 6 & -0.08 & 0.03 & -0.01 & -0.09 & 0.01 & -0.15 & 0.05 & -0.11 & 0.04 \\ 51 & 1 & -0.03 & 0.00 & 0.04 & -0.05 & 0.00 & -0.11 & -0.15 & -0.12 & -0.03 \\ 52 & 1 & 0.00 & 0.00 & -0.03 & -0.07 & 0.01 & -0.07 & -0.12 & 0.00 & -0.07 \\ 53 & 1 & -0.09 & 0.06 & -0.01 & -0.02 & 0.02 & -0.07 & -0.18 & -0.07 & -0.08 \\ 54 & 1 & -0.04 & 0.01 & -0.03 & 0.07 & 0.01 & -0.11 & 0.01 & -0.11 & 0.03 \\ 55 & 1 & -0.02 & 0.03 & 0.00 & 0.04 & 0.01 & -0.12 & 0.13 & -0.15 & 0.03 \\ 56 & 1 & -0.02 & 0.02 & -0.03 & 0.05 & 0.01 & -0.16 & 0.02 & -0.24 & 0.03 \\ 57 & 1 & -0.05 & 0.01 & -0.01 & -0.14 & 0.01 & -0.13 & 0.02 & -0.06 & 0.03 \\ 58 & 1 & -0.08 & 0.00 & 0.03 & -0.09 & 0.00 & -0.20 & 0.03 & -0.18 & 0.03 \\ 59 & 1 & -0.06 & 0.02 & -0.01 & -0.09 & 0.01 & -0.15 & 0.12 & -0.11 & 0.06 \\ 60 & 6 & 0.00 & -0.02 & 0.00 & 0.14 & 0.01 & 0.05 & -0.01 & 0.06 & 0.07 \\ 61 & 1 & -0.05 & -0.05 & -0.02 & 0.12 & 0.00 & 0.05 & 0.01 & 0.07 & 0.06 \\ 62 & 1 & 0.04 & -0.05 & 0.00 & 0.15 & -0.02 & 0.09 & -0.02 & 0.05 & 0.11 \\ 63 & 1 & 0.00 & 0.03 & 0.02 & 0.18 & 0.05 & 0.05 & 0.00 & 0.07 & 0.08\end{array}$

thermodynamics:

Sum of electronic and zero-point Energies=

$-1319.528927$

Sum of electronic and thermal Enthalpies=

$-1319.493620$

Sum of electronic and thermal Free Energies=

$-1319.596479$

Total free energy in solution:

with all non electrostatic terms

$($ a.u. $)=-1320.069725$

Optimized geometry, SCF energy, three lower frequencies, thermochemistry and PCM energy for B3.



\begin{tabular}{|c|c|c|c|c|c|}
\hline \multirow{2}{*}{$\begin{array}{l}\text { Center } \\
\text { Number }\end{array}$} & \multirow{2}{*}{$\begin{array}{l}\text { Atomic } \\
\text { Number }\end{array}$} & \multirow{2}{*}{$\begin{array}{l}\text { Atomic } \\
\text { Type }\end{array}$} & \multicolumn{3}{|c|}{ Coordinates (Angstroms) } \\
\hline & & & $\mathrm{x}$ & Y & Z \\
\hline 1 & 6 & 0 & -0.024483 & -0.007381 & 0.001464 \\
\hline 2 & 6 & 0 & -0.017257 & -0.002082 & 1.399509 \\
\hline 3 & 6 & 0 & 1.194797 & -0.000039 & 2.107522 \\
\hline 4 & 6 & 0 & 2.394184 & 0.001443 & 1.378466 \\
\hline 5 & 6 & 0 & 2.376473 & 0.004348 & -0.019514 \\
\hline 6 & 6 & 0 & 1.169829 & -0.002144 & -0.712215 \\
\hline 7 & 1 & 0 & -0.972948 & -0.008100 & -0.532109 \\
\hline 8 & 1 & 0 & 3.315374 & 0.004427 & -0.569719 \\
\hline 9 & 1 & 0 & 1.160301 & -0.003032 & -1.798181 \\
\hline 10 & 46 & 0 & 1.205839 & -0.007273 & 4.108670 \\
\hline 11 & 6 & 0 & -1.324439 & 0.052846 & 2.153122 \\
\hline 12 & 6 & 0 & 3.713996 & -0.052030 & 2.109361 \\
\hline 13 & 1 & 0 & -1.497194 & -0.862316 & 2.730751 \\
\hline 14 & 1 & 0 & -2.169869 & 0.184466 & 1.473412 \\
\hline 15 & 1 & 0 & 3.895654 & 0.863317 & 2.683818 \\
\hline 16 & 1 & 0 & 4.547763 & -0.183583 & 1.415457 \\
\hline 17 & 6 & 0 & -0.297472 & 1.382455 & 3.947161 \\
\hline 18 & 6 & 0 & -1.728034 & 3.130195 & 4.000394 \\
\hline 19 & 1 & 0 & -2.105475 & 4.091504 & 4.314914 \\
\hline
\end{tabular}






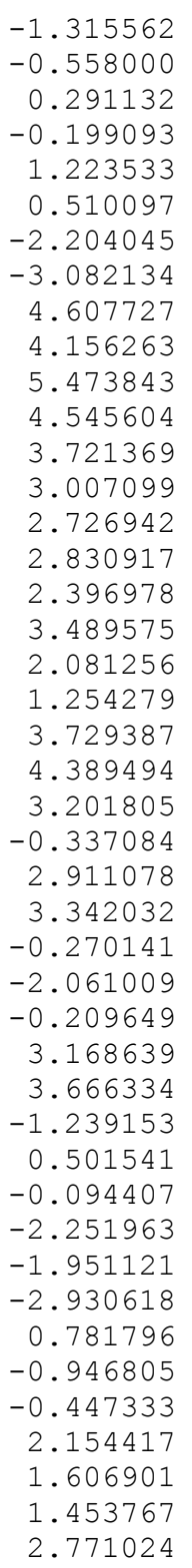

1.187324

2.590333

3. 280926

3. 351670

2.728537

4. 288630

2. 240970

2. 272476

$-2.241907$

$-3.122554$

$-2.274280$

$-4.078040$

$-1.186016$

$-2.573387$

$-1.375044$

1. 229577

0.694124

$-0.182342$

1. 452056

$-0.015299$

$-1.003400$

0.451455

$-0.699781$

$-0.435691$

0.052693

$-1.965578$

1.006309

$-0.216105$

$-2.467750$

$-2.263867$

$-2.748238$

1.003267

0.766549

2.017653

0.825534

$-0.803898$

$-0.598031$

$-2.690660$

$-2.618648$

$-3.160803$

$-3.254038$

$-2.503905$

$-3.918298$

$-3.843225$
3.079102

4.507506

5.460799

6.436638

5.558440

5.097127

3.091989

2. 464960

3.031096

3. 960606

2. 387752

4.277963

3. 036081

4.501183

3. 932161

6.045256

6.898420

7.514744

7. 631376

6.407037

6.829853

7.568905

8.893393

7.603491

9.629649

9. 291815

9.186214

6.358532

8.261512

10.322362

8.608164

9.697292

9.921974

8.807604

6.089365

5.442859

6.905431

8.661237

9. 058311

7.449018

5.459579

6.027476

4. 944182

6.142263

$\mathrm{HF}=-1320.1121591$

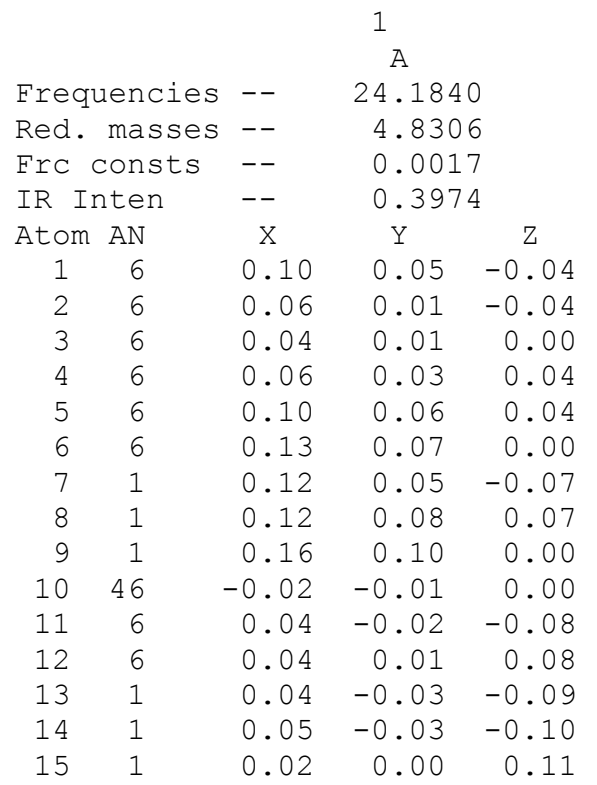

2

33.3584

4. 1634

0.0027

0.1937

$\mathrm{X}$

0.04

0.02

0.01

0.02

0.04

0.05

0.05

0.05

0.07

$-0.02$

0.01

0.01

0.01

0.02

0.02
$\mathrm{Y}$

$0.09-0.04$

$0.04-0.04$

$0.01-0.02$

$0.03 \quad 0.00$

$0.08 \quad 0.00$

$0.11-0.02$

$0.11-0.06$

$0.10 \quad 0.01$

$0.15-0.02$

$-0.02-0.02$

$0.01-0.06$

$0.00 \quad 0.01$

$-0.01-0.08$

$0.02-0.07$

$\begin{array}{rr}-0.02 & 0.04\end{array}$
3

A

38.0190

3.9756

0.0034

0.1613

$\mathrm{X}$

0.01

$\mathrm{Y}$

0.05

0.04

Z

0.00

$0.00-0.02 \quad 0.01$

$\begin{array}{llll}0.01 & -0.01 & 0.01\end{array}$

$\begin{array}{lll}0.01 & 0.03 & 0.00\end{array}$

$\begin{array}{lll}0.01 & 0.08 & 0.00\end{array}$

$\begin{array}{llll}0.01 & -0.04 & 0.01\end{array}$

$\begin{array}{lll}0.01 & 0.03 & 0.00\end{array}$

$\begin{array}{lll}-0.01 & 0.01 & 0.00\end{array}$

$\begin{array}{llll}0.00 & 0.07 & -0.01\end{array}$

$\begin{array}{lll}0.00 & -0.06 & 0.01\end{array}$

$\begin{array}{llll}-0.02 & 0.07 & -0.01\end{array}$

$\begin{array}{llll}0.01 & 0.08 & -0.01\end{array}$

$\begin{array}{lrr}0.03 & -0.06 & 0.00\end{array}$ 


\begin{tabular}{|c|c|c|c|c|c|c|c|c|c|c|}
\hline 16 & 1 & 0.06 & 0.02 & 0.10 & 0.02 & 0.00 & 0.02 & 0.00 & -0.08 & 0.01 \\
\hline 17 & 6 & -0.04 & -0.02 & -0.03 & -0.02 & -0.02 & -0.01 & 0.02 & 0.04 & 0.01 \\
\hline 8 & 6 & -0.08 & -0.06 & -0.05 & -0.04 & -0.04 & 0.01 & 0.03 & 0.06 & 0.02 \\
\hline 19 & 1 & -0.11 & -0.07 & -0.04 & -0.05 & -0.05 & 0.03 & 0.04 & 0.06 & 0.02 \\
\hline 20 & 7 & -0.01 & -0.03 & -0.06 & -0.01 & -0.01 & -0.03 & 0.02 & 0.06 & 0.00 \\
\hline 21 & 7 & -0.09 & -0.04 & -0.02 & -0.04 & -0.04 & 0.01 & 0.02 & 0.04 & 0.02 \\
\hline 22 & 6 & -0.13 & -0.03 & 0.02 & -0.05 & -0.05 & 0.03 & 0.03 & 0.03 & 0.02 \\
\hline 23 & 1 & -0.17 & -0.04 & 0.00 & -0.06 & -0.07 & 0.03 & 0.04 & 0.00 & 0.03 \\
\hline 24 & 1 & -0.13 & -0.02 & 0.05 & -0.05 & -0.05 & 0.03 & 0.04 & 0.04 & 0.00 \\
\hline 25 & 1 & -0.13 & -0.02 & 0.03 & -0.05 & -0.04 & 0.05 & 0.01 & 0.04 & 0.04 \\
\hline 26 & 6 & -0.04 & -0.05 & -0.07 & -0.02 & -0.02 & -0.01 & 0.03 & 0.07 & 0.01 \\
\hline 27 & 1 & -0.02 & -0.07 & -0.10 & -0.01 & -0.02 & -0.02 & 0.03 & 0.08 & 0.00 \\
\hline 28 & 6 & -0.03 & -0.03 & 0.05 & -0.03 & -0.03 & -0.03 & -0.06 & -0.08 & 0.02 \\
\hline 29 & 6 & -0.07 & -0.05 & 0.01 & -0.05 & -0.05 & -0.05 & -0.08 & -0.05 & 0.03 \\
\hline 30 & 1 & -0.01 & -0.03 & 0.08 & -0.02 & -0.03 & -0.02 & -0.06 & -0.10 & 0.02 \\
\hline 31 & 1 & -0.11 & -0.07 & 0.00 & -0.06 & -0.06 & -0.06 & -0.10 & -0.06 & 0.04 \\
\hline 32 & 7 & 0.00 & -0.01 & 0.06 & -0.01 & -0.02 & -0.01 & -0.03 & -0.06 & 0.01 \\
\hline 33 & 7 & -0.08 & -0.04 & -0.01 & -0.04 & -0.04 & -0.05 & -0.06 & -0.02 & 0.03 \\
\hline 34 & 6 & -0.03 & -0.01 & 0.02 & -0.02 & -0.03 & -0.03 & -0.04 & -0.02 & 0.01 \\
\hline 35 & 1 & -0.05 & 0.05 & -0.02 & 0.06 & -0.11 & -0.09 & -0.05 & 0.06 & 0.00 \\
\hline 36 & 6 & -0.01 & 0.01 & -0.02 & 0.03 & -0.03 & -0.06 & -0.03 & 0.05 & 0.00 \\
\hline 37 & 6 & 0.03 & 0.00 & -0.11 & -0.01 & -0.04 & 0.02 & 0.01 & 0.10 & 0.00 \\
\hline 38 & 1 & 0.02 & -0.02 & 0.02 & 0.05 & 0.03 & -0.11 & -0.07 & 0.04 & -0.01 \\
\hline 39 & 8 & -0.02 & 0.02 & 0.00 & 0.01 & -0.02 & -0.02 & 0.00 & -0.01 & 0.00 \\
\hline 40 & 1 & 0.02 & 0.04 & -0.16 & -0.09 & -0.14 & 0.11 & 0.06 & 0.11 & 0.00 \\
\hline 41 & 1 & 0.02 & 0.02 & -0.11 & 0.04 & -0.10 & -0.08 & -0.02 & 0.15 & 0.00 \\
\hline 42 & 6 & 0.09 & -0.07 & -0.12 & -0.01 & 0.15 & 0.09 & 0.05 & 0.09 & 0.00 \\
\hline 43 & 50 & 0.01 & 0.03 & 0.04 & 0.04 & 0.00 & 0.03 & 0.00 & -0.05 & -0.02 \\
\hline 44 & 1 & 0.07 & -0.12 & -0.08 & 0.10 & 0.28 & 0.02 & -0.02 & 0.08 & 0.00 \\
\hline 45 & 6 & 0.16 & -0.09 & -0.19 & -0.15 & 0.18 & 0.23 & 0.15 & 0.11 & 0.01 \\
\hline 46 & 6 & 0.05 & 0.04 & 0.03 & 0.07 & 0.01 & 0.01 & -0.11 & 0.03 & -0.08 \\
\hline 47 & 6 & -0.01 & 0.02 & 0.06 & 0.02 & 0.00 & 0.05 & 0.03 & -0.22 & -0.10 \\
\hline 48 & 6 & 0.03 & 0.04 & 0.07 & 0.02 & 0.00 & 0.03 & 0.11 & 0.01 & 0.12 \\
\hline 49 & 1 & 0.20 & -0.14 & -0.20 & -0.15 & 0.32 & 0.27 & 0.17 & 0.10 & 0.02 \\
\hline 50 & 1 & 0.18 & -0.04 & -0.23 & -0.28 & 0.07 & 0.31 & 0.22 & 0.13 & 0.02 \\
\hline 51 & 1 & 0.07 & 0.04 & 0.06 & 0.07 & 0.05 & 0.01 & -0.11 & -0.01 & -0.09 \\
\hline 52 & 1 & 0.08 & 0.05 & 0.00 & 0.07 & 0.00 & 0.01 & -0.09 & 0.10 & -0.07 \\
\hline 53 & 1 & 0.03 & 0.04 & 0.01 & 0.10 & 0.00 & -0.01 & -0.16 & 0.02 & -0.12 \\
\hline 54 & 1 & -0.02 & 0.02 & 0.07 & 0.01 & 0.00 & 0.06 & -0.06 & -0.24 & -0.10 \\
\hline 55 & 1 & -0.01 & 0.03 & 0.06 & 0.02 & 0.01 & 0.04 & 0.14 & -0.20 & -0.10 \\
\hline 56 & 1 & 0.00 & 0.00 & 0.06 & 0.03 & -0.01 & 0.05 & 0.05 & -0.32 & -0.14 \\
\hline 57 & 1 & 0.06 & 0.04 & 0.00 & 0.01 & -0.01 & 0.03 & 0.14 & 0.08 & 0.09 \\
\hline 58 & 1 & 0.09 & 0.07 & 0.14 & 0.02 & 0.00 & 0.03 & 0.16 & 0.04 & 0.17 \\
\hline 59 & 1 & -0.04 & 0.03 & 0.11 & 0.01 & 0.00 & 0.03 & 0.10 & -0.07 & 0.19 \\
\hline 60 & 6 & -0.12 & -0.04 & -0.05 & -0.06 & -0.05 & -0.06 & -0.07 & 0.01 & 0.04 \\
\hline 61 & 1 & -0.11 & -0.04 & -0.04 & -0.07 & -0.05 & -0.07 & -0.03 & 0.03 & 0.05 \\
\hline 62 & 1 & -0.13 & 0.00 & -0.09 & -0.05 & -0.05 & -0.08 & -0.11 & 0.04 & 0.06 \\
\hline 63 & 1 & -0.16 & -0.08 & -0.05 & -0.06 & -0.05 & -0.06 & -0.08 & -0.01 & 0.03 \\
\hline
\end{tabular}

thermodynamics:

Sum of electronic and zero-point Energies=

$-1319.584847$

Sum of electronic and thermal Enthalpies=

$-1319.549486$

Sum of electronic and thermal Free Energies=

$-1319.651612$

Total free energy in solution:

with all non electrostatic terms

$(\mathrm{a} \cdot \mathrm{u})=$.

\section{Reference 48}

Gaussian 03 Revision C.02, M. J. Frisch, G. W. Trucks, H. B. Schlegel, G. E. Scuseria, M. A. Robb, J. R. Cheeseman, J. A. Montgomery, Jr., T. Vreven, K. N. Kudin, J. C. Burant, J. M. Millam, S. S. Iyengar, J. Tomasi, V. Barone, B. Mennucci, M. Cossi, G. Scalmani, N. Rega, 
G. A. Petersson, H. Nakatsuji, M. Hada, M. Ehara, K. Toyota, R. Fukuda, J. Hasegawa, M. Ishida, T. Nakajima, Y. Honda, O. Kitao, H. Nakai, M. Klene, X. Li, J. E. Knox, H. P. Hratchian, J. B. Cross, V. Bakken, C. Adamo, J. Jaramillo, R. Gomperts, R. E. Stratmann, O. Yazyev, A. J. Austin, R. Cammi, C. Pomelli, J. W. Ochterski, P. Y. Ayala, K. Morokuma, G. A. Voth, P. Salvador, J. J. Dannenberg, V. G. Zakrzewski, S. Dapprich, A. D. Daniels, M. C. Strain, O. Farkas, D. K. Malick, A. D. Rabuck, K. Raghavachari, J. B. Foresman, J. V. Ortiz, Q. Cui, A. G. Baboul, S. Clifford, J. Cioslowski, B. B. Stefanov, G. Liu, A. Liashenko, P. Piskorz, I. Komaromi, R. L. Martin, D. J. Fox, T. Keith, M. A. Al-Laham, C. Y. Peng, A. Nanayakkara, M. Challacombe, P. M. W. Gill, B. Johnson, W. Chen, M. W. Wong, C. Gonzalez, and J. A. Pople, Gaussian, Inc., Wallingford CT. 